\title{
The characterisation and application of bacterial nitroreductase enzymes
}

By

\author{
Sarah Condon
}

A thesis submitted to Victoria University of Wellington in fulfilment of the requirements for the degree of

Master of Science

in Cell and Molecular Bioscience

Victoria University of Wellington

(2013) 


\begin{abstract}
Cancer is an increasing global concern, with the number of people diagnosed growing rapidly each year. Gene directed enzyme prodrug therapy (GDEPT) is emerging as a front-runner of new technologies that seek to combat the growing number of cases. One developing approach to GDEPT involves the use of bacterial nitroreductase enzymes to reduce prodrug substrates, which, upon reduction to their active form, are toxic to cancer cells through DNA crosslinking.
\end{abstract}

Nitroreductases have the ability to activate a variety of nitro-quenched compounds, not only anti-cancer prodrugs, but also nil bystander antibiotics and masked fluorophores, through the reduction of strongly electron-withdrawing nitro substituents on aromatic rings. My research initially sought to exploit this capability by partnering nitroreductases with nil bystander antibiotics for targeted cell ablation, as a component of a larger gene directed enzyme prodrug therapy project. This has potential to provide important safety features for removal of viral and bacterial vectors following anti-cancer gene therapy.

From this, the main focus evolved into utilising nitroreductase enzymes for targeted cell ablation for applications in developmental and regenerative biology. This exploited the ability of nitroreductases to activate nil bystander antibiotics in partnership with masked fluorophores for imaging purposes. It has previously been shown that antibiotics can be applied to a nitroreductase under control of a tissuespecific promoter in a transgenic model organism, enabling controlled ablation of that tissue at precise stages of development. However, direct imaging of the nitroreductase location and activity, by application of masked fluorophore probes prior to ablation, has not previously been explored.

During the course of this work, several promising combinations of nitroreductases that exhibit opposing specificities for certain combinations of masked fluorophores and nil-bystander antibiotics were identified through screening in bacterial systems. In general, these results were found to translate effectively into eukaryotic cell lines. Pairs of nitroreductases that have opposite specificities for two different antibiotic substrates offer potential for the multiplexed ablation of either (or both) of two 
different labelled tissues in the same transgenic model organism, according to the substrate(s) administered to that organism.

Throughout this screening process, a nitroaromatic substrate (niclosamide) was identified that is, uniquely, initially toxic to Escherichia coli but becomes non-toxic upon reduction of the nitro substituent. Using niclosamide, a novel strategy with potential for identification of new nitroreductases, as well as selection-based directed evolution to improve desired activities, was explored. 


\section{Acknowledgements}

Firstly I would like to thank my supervisor David Ackerley, for always being able to help with this project, and the ability to envision a clear final goal for experimental work, seeing the bigger picture and applying what you are doing to this kind of context was always hugely motivating. And thank you for putting in the enourmous effort of proof reading and revision of this thesis.

I am also extremely grateful for our collaborators at the Auckland Cancer Research Society Centre for the use of compounds synthesised there, and new ideas for experimental work. Without them my project would not have evolved in the way it has as the use of their masked fluorophores has expanded the range of my thesis greatly.

I would like to thank everyone in the Ackerley lab. Working in the lab was always a good time, and anytime I needed help with anything, I knew that there would be plenty of people more than willing to help. In particular I would like to say a big thanks to Janine and Elsie, who first showed me my way around the bacterial and working with nitroreductase enzymes - your knowledge and continuing experimental guidance has been a huge help throughout this process and much appreciated.

Also, a big thanks to Claire who, with minimal time to complete her thesis, showed me the ins and outs of tissue culture in limited time. Varun and Amy have also been especially helpful when it has come to all things tissue culture, without you guys I don't think I would've got very far with any work that needed to be done in tissue culture, so thanks.

Finally I would like to thank friends and family for always being around when I needed a distraction; encouraging me to take breaks when they thought I'd been working too hard, or enabling me to focus when I needed too, and always with words of encouragement.

Thank you to everyone who has helped in any way with the completion of this thesis; every little bit has been appreciated. 


\section{Table of Contents}

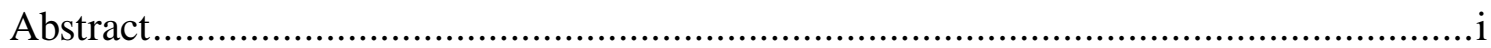

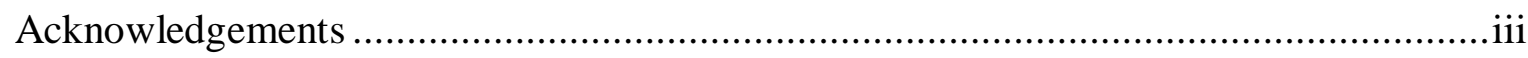

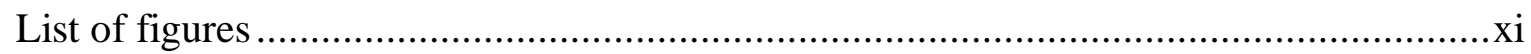

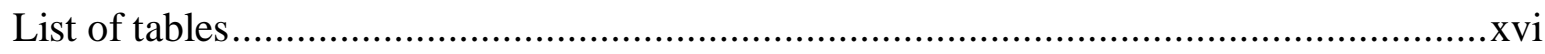

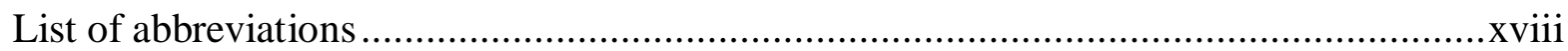

Preface

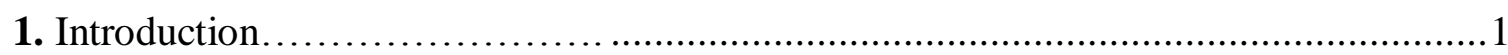

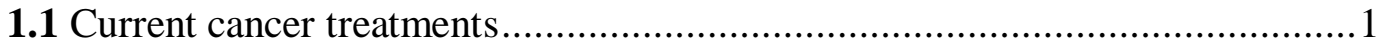

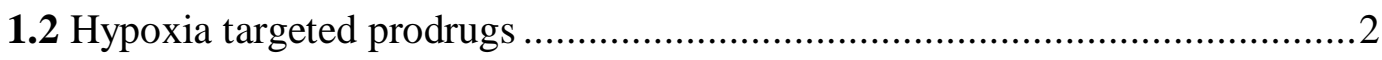

1.2.1 Nitroaromatic hypoxia activated prodrugs ...................................4

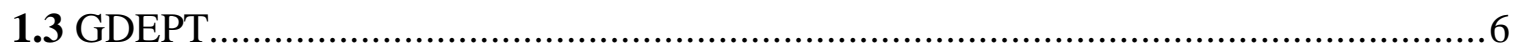

1.3.1 E. coli nitroreductase (NfsB) and 5-aziridin-1-yl-2-4- dinitrobenzamide

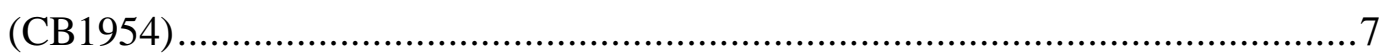

1.4 The 58 -membered nitroreductase library .....................................................

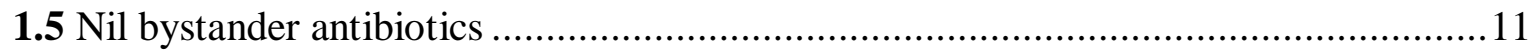

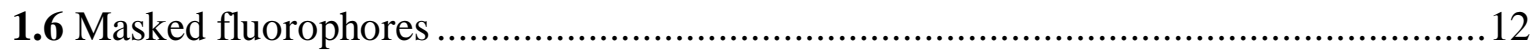

1.7 Regenerative and developmental biological studies.......................................... 13

1.7.1 Case studies utilising nitroreductase ablation technology .........................15

1.7.2 Limitations of the NfsB_Ec/ metronidazole system ................................17 
1.7.4 Dual reporter/ ablation system exploiting the characteristics of nitroreductases

1.8 Aims 19

2. Materials and Methods 21

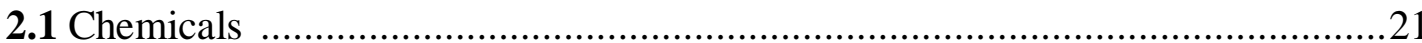

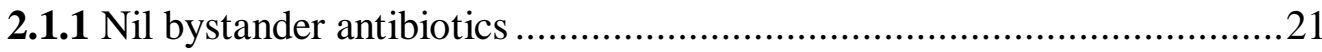

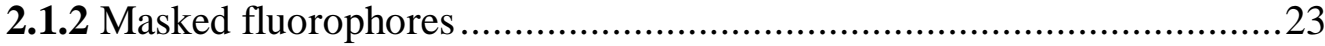

2.2 Enzymes

2.3 Bacterial strains and plasmids .............................................................26

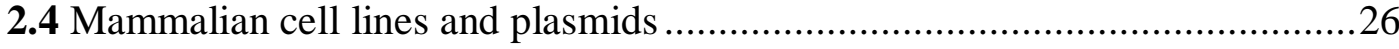

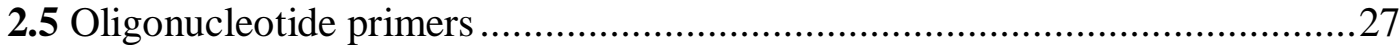

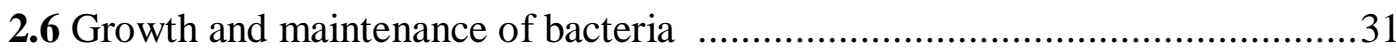

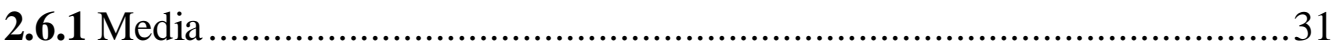

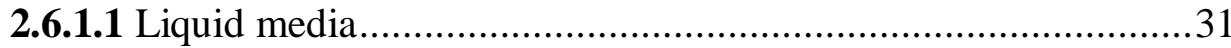

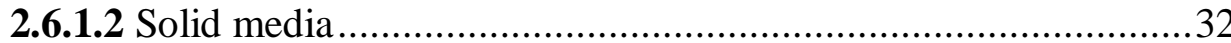

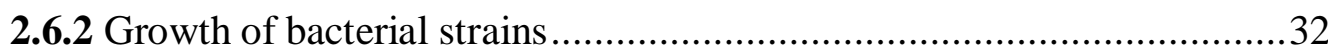

2.7 Growth and maintenance of mammalian cell lines.........................................32

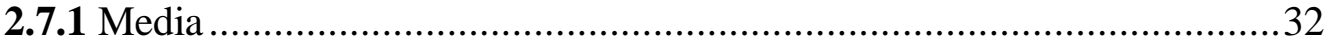

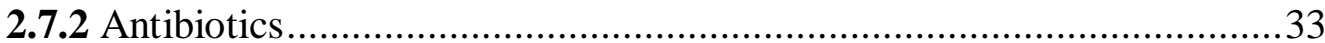

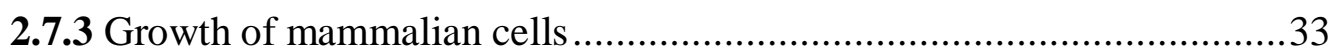


2.8.1 Chemically competent cells 34

2.8.2 Transformation protocol 35

2.8.3 Electrocompetent cells 35

2.8.4 Electroporation protocol 35

2.8.5 Colony screening 36

2.8.6 DNA quantification 36

2.8.7 Polymerase chain reaction (PCR) .................................................36

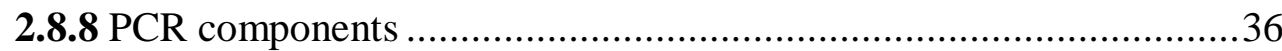

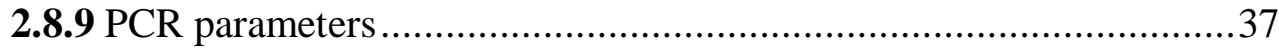

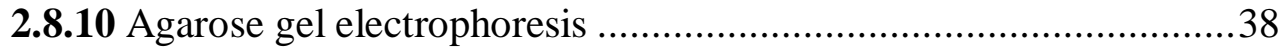

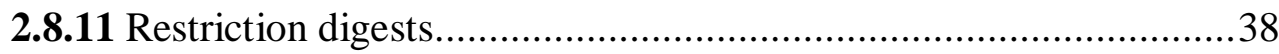

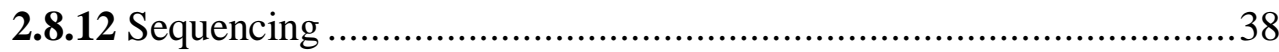

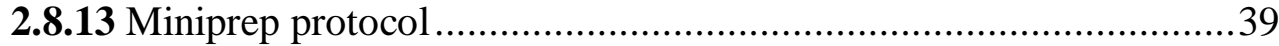

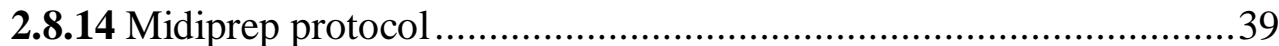

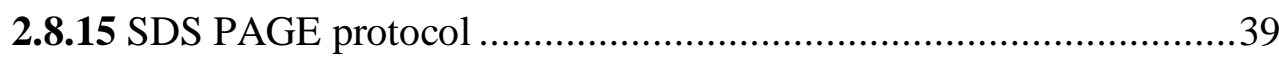

2.8.15.1 Recipes for SDS PAGE gels and buffers ..............................40

2.9 Bacterial cell protocols ..................................................................... 41

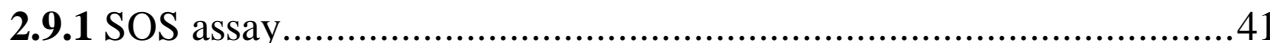


2.9.2 Growth inhibition assay 42

2.9.3 $\mathrm{IC}_{50}$ analysis 42

2.9.4 Fluorescence assay .43

2.9.5 Combined growth inhibition and fluorescence assays 44

2.9.6 Bacterial confocal microscopy 44

2.9.7 Validation of liquid media protocol with potential to be used for novel nitroreductase discovery

2.9.8 Validation of solid media protocol with potential to be used for novel nitroreductase discovery .45

2.10 Mammalian cell protocols .45

2.10.1 Construction of mammalian NTR stable expression cell lines 45

2.10.2 Creating Gateway® constructs 45

2.10.3 BP recombination 46

2.10.4 Analysing entry clones .46

2.10.5 LR recombination 46

2.10.6 Confirming the identity of F279/ V5 destination clones 47

2.10.7 Stable Transfection Cell Lines .47

2.10.7.1 Lipofectamine ${ }^{\mathrm{TM}} 2000$ reagent protocol 47

2.10.7.2 Making stocks of stable cell lines 48

2.10.8 MTS assay..... 48

2.10.9 Confocal microscopy in mammalian cells .49 
2.10.10 Microscopy of challenged mammalian cells 49

2.10.11 Confocal microscopy of challenged mammalian cells treated with masked fluorophores

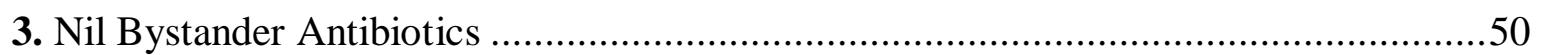

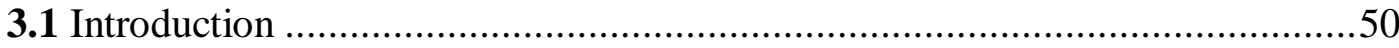

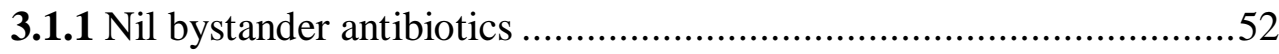

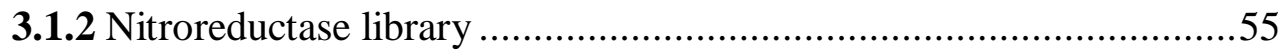

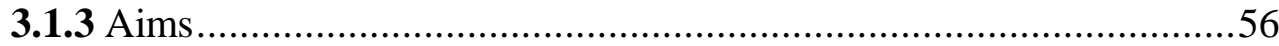

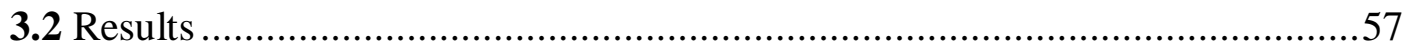

3.2.1 Identification of nitroreductases with a superior activation profile with metronidazole than NfsB_Ec ......................................................57

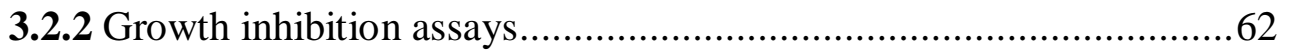

3.2.3 Identification of pairs of nitroreductases / nil bystander antibiotics with opposing activation profiles .66

3.2.4 Nitroreductase mediated cell ablation in human cell lines ...............71

3.2.5 Gateway $^{\mathrm{TM}}$ recombination system …..................................... 72

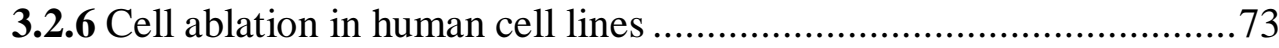

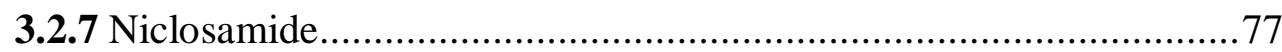

3.2.8 Exploiting the characteristics of niclosamide ................................78

3.2.8.1 Optimisation of niclosamide selection in liquid media .......78

3.2.8.2 Optimisation of niclosamide selection in solid media .82 
3.3 Discussion. .85

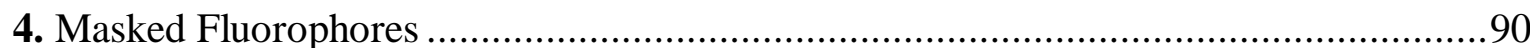

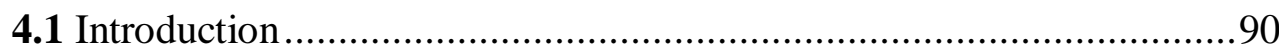

4.1.1 Masked fluorophores ...................................................91

4.1.2 Fluorescent reporter proteins ..........................................91

4.1.3 Dual ablation/ reporter system in developmental biology......92

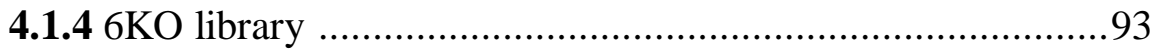

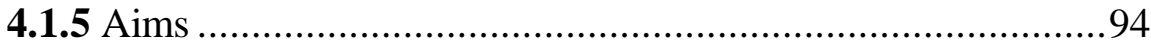

4.2 Results

4.2.1 Fluorescent screening in bacterial cells.............................94

4.2.2 Combined fluorescence reporting and cell ablation..............97

4.2.3 Dual fluorophore screening in bacterial cells ......................101

4.2.4 Dual fluorophore screening in human cell lines ...................105

4.2.5 Dual fluorescence reporting/ nil bystander antibiotic targeted cell ablation ............................................................ 110

4.3 Discussion 117

5. Summary, Conclusions, and Future Directions 120

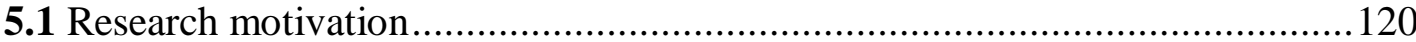

5.1.1 GDEPT 120

5.1.2 Novel developmental biology tools 121

5.2 Key Findings 
5.2.1 Identification of nitroreductases active with nil bystander antibiotics

5.2.2 Identification of nitroreductases superior to NfsB_Ec for metronidazole activation 123

5.2.3 Combined dual reporter/ ablation system 124

5.2.4 Niclosamide 126

5.3 Future Directions 126

5.3.1 GDEPT 126

5.3.2 Developmental and regenerative biological studies 127

5.3.3 Niclosamide as a tool for directed evolution 128

5.4 Concluding remarks 128

References 130 


\section{List of Figures}

Figure 1.1 Hypoxia prodrug mechanism of activation ..........................................4

Figure 1.2 Hypoxia activation of nitroaromatic prodrug ......................................4

Figure 1.3 Structures of nitroaromatic anti-cancer compounds .............................5

Figure 1.4 Hypoxia activated nitroaromatic prodrug …........................................

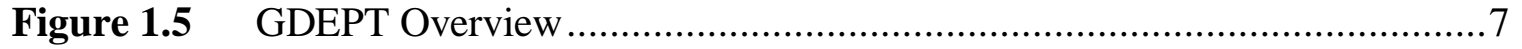

Figure 1.6 Dr Adam Patterson, University of Auckland, unpublished work.................11

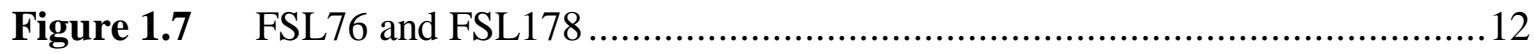

Figure 1.8 Bioreductive trigger molecule components........................................13

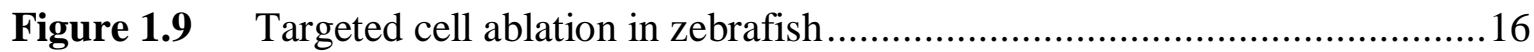

Figure 1.10 Live imaging of oligodendrocyte depletion and reappearance following

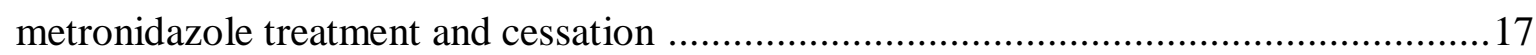

Figure 1.11 Nitroreductases with opposing specificities for both masked

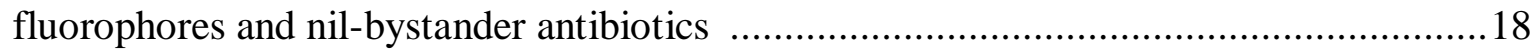

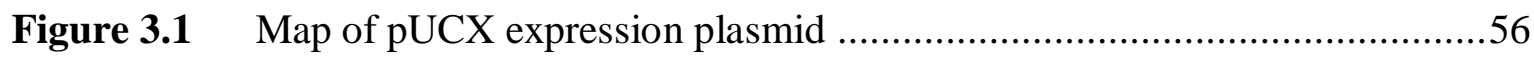

Figure 3.2 Metabolism of metronidazole by members of the 58 nitroreductase over-expression library as measured by growth inhibition assay ..................................58

Figure 3.3 Metabolism of metronidazole by 6 nitroreductase enzymes from the 58 nitroreductase enzyme over-expression library over a range of concentrations as measured by $\mathrm{IC}_{50}$ analysis .59

Figure 3.4 Metronidazole challenged HCT-116: NfsB_Vh cells. 61 
Figure 3.5 Metronidazole challenged HEK293: NfsB_Ec cells 62

Figure 3.6 Colour coded heat-map summarising activation profiles of nitroreductase/ nil bystander antibiotic combination 64

Figure 3.7 Identification of nitroreductase enzymes that exhibit opposing activation profiles with tinidazole and metronidazole 66

Figure 3.8 Identification of nitroreductase enzymes that exhibit opposing activation profiles with F-misonidazole and metronidazole

Figure 3.9 Identification of nitroreductase enzymes that exhibit opposing activation profiles with EF5 and dimetridazole

Figure 3.10 Identification of nitroreductase enzymes that exhibit opposing activation profiles with $\mathrm{EF} 5 /$ and metronidazole

Figure 3.11 Metabolism of metronidazole and tinidazole by E. coli cells overexpressing nitroreductase NfsA_Li over a range of concentrations as measured by $\mathrm{IC}_{50}$ analysis

Figure 3.12 Metabolism of metronidazole and tinidazole by $E$. coli cells overexpressing nitroreductase NfsA_Pp over a range of concentrations as measured by $\mathrm{IC}_{50}$ analysis 68

Figure 3.13 Metabolism of metronidazole and tinidazole by $E$. coli cells overexpressing nitroreductase NfsA_Ecaro over a range of concentrations as measured by $\mathrm{IC}_{50}$ analysis

Figure 3.14 Metabolism of metronidazole and tinidazole by $E$. coli cells overexpressing nitroreductase $\mathrm{Nfs} \mathrm{B}_{-} \mathrm{Vv}$ over a range of concentrations as measured by $\mathrm{IC}_{50}$ analysis .70

Figure 3.15 Overview of the Gateway ${ }^{\mathrm{TM}}$ recombination system. 73

Figure 3.16 Metronidazole and tinidazole challenged HEK293 WT cells .74

Figure 3.17 Metronidazole and tinidazole challenged HEK293: Nfs A_Pp cells..... .75 
Figure 3.18 Metronidazole and tinidazole challenged HEK293: NfsB_Vv cells 76

Figure 3.19 Metabolism of metronidazole or niclosamide by members of the 58 nitroreductase over-expression library as measured by growth inhibition assay .78

Figure 3.20 Metabolism of niclosamide by E.coli $6 \mathrm{KO}$ overexpressing NfsA_Vv or pUCX over an $8 \mathrm{~h}$ time period

Figure 3.21 Metabolism of niclosamide by E.coli $7 \mathrm{KO}$ overexpressing NfsA_Vv or pUCX over an $8 \mathrm{~h}$ time period

Figure 3.22 Metabolism of niclosamide by E.coli SOS-R2 overexpressing

NfsA_Vv or pUCX over an $8 \mathrm{~h}$ time period 81

Figure 3.23 PCR confirmation of active nitroreductase enzymes from colonies picked from mixed culture NfsA_Vv/ pUCX plated on media containing metronidazole and niclosamide.

Figure 3.24 FSL41 fluorescence visualised on UV trans-illuminator as confirmation of active nitroreductase enzymes from colonies picked from mixed culture NfsA_Vv/ pUCX plated on media containing metronidazole and niclosamide .84

Figure 3.25 FSL41 fluorescence plate reader ex $355 \mathrm{~nm} / \mathrm{em} 460 \mathrm{~nm}$ values as confirmation of active nitroreductase enzymes from colonies picked from mixed culture NfsA_Vv/ pUCX plated on media containing metronidazole and niclosamide .85

Figure 4.1 Heat-map of 23 nitroreductase 6KO library with different masked fluorophores 96

Figure 4.2 UV Trans-illuminator image of FSL41-treated cultures of E. coli $6 \mathrm{KO}$ over-expressing different candidate nitroreductases, with (top) or without (bottom) antibiotic challenge

Figure 4.3 6KO E. coli empty plasmid (pUCX) control strain treated with FSL41 ....98

Figure 4.4 6KO E. coli over-expressing NfsB_Vh, treated with FSL41 .98 
Figure 4.5 6KO E. coli over-expressing NfsB_Vh treated with FSL41 and metronidazole .99

Figure 4.6 HCT116: NfsB_Vh treated with FSL41 100

Figure 4.7 HCT116: NfsB_Vh treated with FSL41 and challenged with metronidazole 100

Figure 4.8 Identification of candidate nitroreductase enzymes that exhibit opposing activation profiles for two nil bystander antibiotics, and two masked fluorophores 102

Figure 4.9 6KO E. coli over-expressing NfsA_Pp treated with FSL76 102

Figure 4.10 6KO E. coli over-expressing NfsA_Pp treated with FSL178 103

Figure 4.11 6KO E. coli over-expressing NfsB_Vv treated with FSL178 103

Figure 4.12 6KO E. coli over-expressing NfsB_Vv treated with FSL76 103

Figure 4.13 6KO E. coli mixed culture multiplex fluorescence imaging 104

Figure 4.14 6KO E. coli pUCX treated with FSL76 and FSL178 104

Figure 4.15 HEK293 WT cells treated with either FSL41, FSL76, or FSL178 106

Figure 4.16 HEK293: NfsB_Ec treated with FSL41 106

Figure 4.17 HEK293: NfsB_Vv treated with FSL41 107

Figure 4.18 HEK293: NfsA_Pp treated with FSL41 107

Figure 4.19 HEK293: NfsA_Pp treated with FSL76 108

Figure 4.20 HEK293: NfsA_Pp treated with FSL178 ............................................108

Figure 4.21 HEK293: NfsB_Vv treated with FSL178 109

Figure 4.22 HEK293: NfsB_Vv treated with FSL76 109 
Figure 4.23 HEK293 mixed culture multiplex fluorescence imaging

Figure 4.24 6KO E. coli over-expressing NfsA_Pp treated with FSL76 and challenged with metronidazole

Figure 4.25 6KO E. coli over-expressing NfsA_Pp treated with FSL76 and challenged with tinidazole

Figure 4.26 6KO E. coli over-expressing NfsB_Vv treated with FSL178 and challenged with tinidazole

Figure 4.27 6KO E. coli over-expressing NfsB_Vv treated with FSL178 and challenged with metronidazole

Figure 4.28 6KO E. coli mixed culture multiplex fluorescence imaging and ablation challenged with metronidazole

Figure 4.29 6KO E. coli mixed culture multiplex fluorescence imaging and ablation challenged with tinidazole

Figure 4.30 6KO E. coli mixed culture multiplex fluorescence imaging and ablation challenged with metronidazole and tinidazole

Figure 4.31 HEK293: NfsA_Pp treated with FSL76 and challenged with tinidazole.....115

Figure 4.32 HEK293: NfsB_Vv treated with FSL178 and challenged with metronidazole 115

Figure 4.33 HEK293 mixed culture multiplex fluorescence imaging and ablation challenged with metronidaazole 116

Figure 4.34 HEK293 mixed culture multiplex fluorescence imaging and ablation challenged with tinidazole 


\section{List of Tables}

Table 1.1 Complete 58 nitroreductase library .....................................................

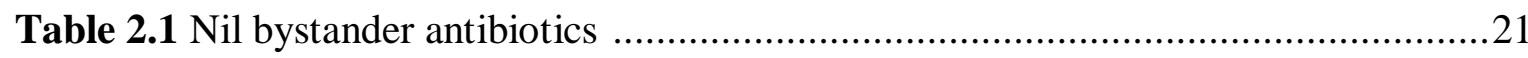

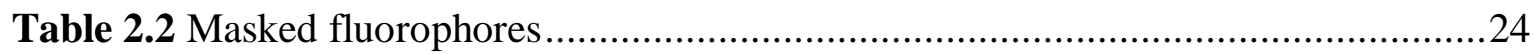

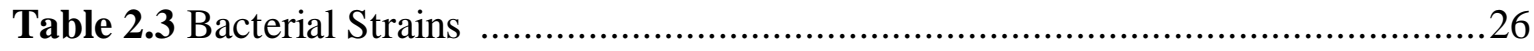

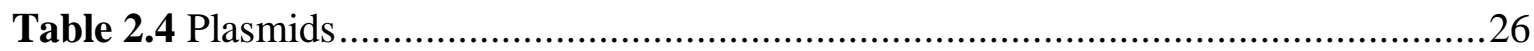

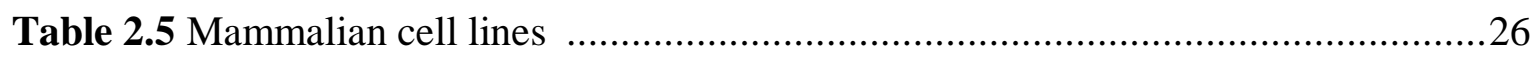

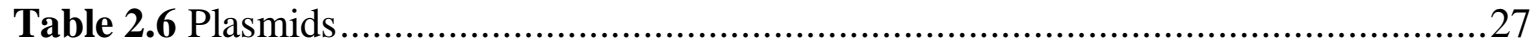

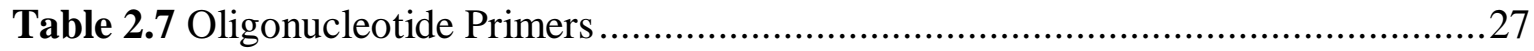

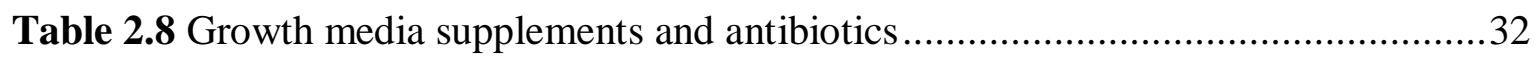

Table 2.9 Antibiotics used in the growth of mammalian cell lines ...............................33

Table 2.10 Recipes for chemically competent cell buffers ...........................................

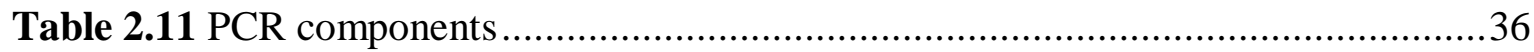

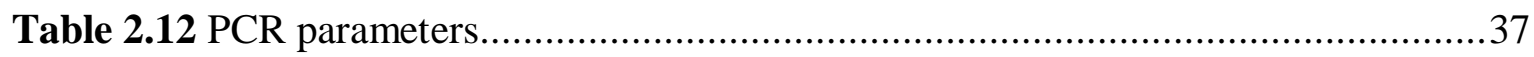

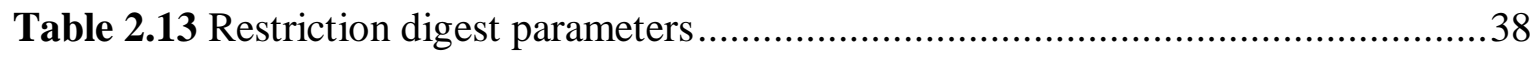

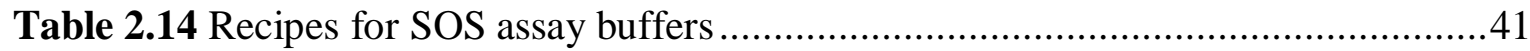

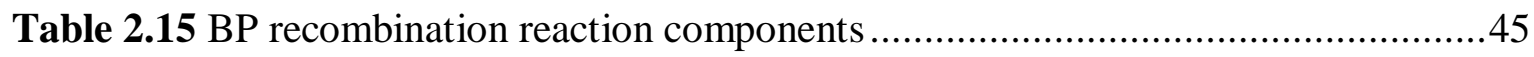

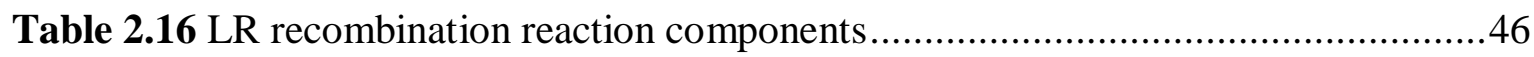

Table 3.1 IC ${ }_{50}$ results of 6 nitroreductase enzymes from the 58 nitroreductase

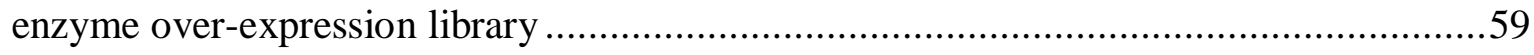


Table 4.1 23 nitroreductase 6KO library

.93 


\section{List of Abbreviations}

2M5NI 2-Methyl-5-Nitroimidazole

4NI 4-Nitroimidazole

ACSRC Auckland Cancer Society Research Centre

Amp Ampicillan

BRT Bioreductive Trigger

CB1954 5-aziridn-1-yl-2-4- dinitrobenzamide

DMEM Dulbeccos Modified Eagle Medium

DMSO Dimethylsulfoxide

DNBM Dinitrobenzamide Mustard

DTZ Dimetridazole

FCS Fetal Calf Serum

FMISO F-Misonidazole

FUR Furazolidone

GDEPT Gene Directed Enzyme Prodrug Therapy

HEPES 4-(2-hydroxyethyl)-1-piperazineethanesulfonic acid

$\mathbf{I C}_{50} \quad$ Half Maximal Inhibitory Concentration

Kan Kanamycin

LB Lauria Broth

MISO Misonidazole

MTZ Metronidazole

NB2N $N$-Benznidazole

NCS Niclosamide

NFN Nitrofurantoin

NFX Nifurtimox

NFZ Nitrofurazone

NTR Nitroredustase

NQO1 NAD $(\mathrm{P}) \mathrm{H}$ : quinone oxidoreductase 1

OD Optical Density (at given $\lambda$ )

ORN Ornidazole

PCR Polymerase Chain Reaction

RNZ Ronidazole 
Tinidazole

WT

Wild Type 


\section{Preface}

At the time that this project started, previous work in the Ackerley lab had focused on developing a nitroreductase-based strategy for GDEPT, utilising the ability of nitroreductase enzymes to activate nitro-quenched compounds. In this scenario, it was proposed that a single nitroreductase enzyme would be capable of reducing a PET imaging agent, for confirmation of localisation of the nitroreductase containing vector, a prodrug, capable of eliminating the target tumour cell population, and a nil bystander antibiotic, a safety mechanism for the ablation of the vector delivery system post treatment. My initial work was focused on identifying nil bystander antibiotic/ nitroreductase combinations that might enable vector ablation. However, this rapidly evolved into the main focus of this thesis; exploring the potential for use of nil bystander antibiotics/ nitroreductases for targeted cell ablation in developmental biology. Acknowledging this progression, the Introduction will first examine cancer therapy as a means of introducing key enzymes, strategies and compounds, but will then move onto other applications of the ability of nitroreductases to activate nitroquenched molecules.

\section{Introduction}

\subsection{Current cancer treatments}

Due the multi-factorial nature of cancer, it is a difficult disease to treat in each individual. Cancer tumours are, essentially, an uncontrolled growth of cells due to the inability to regulate tissue growth (Croce, 2008). The rapid proliferation of solid tumours causes blood vessels to be forced apart, to have impaired function due to cellular mutations, or to be produced at a high enough rate resulting in the inability to maintain homeostatic regulation of tissue and blood pressure formation (Brown and Giaccia, 1998) resulting in a poorly organised vascular architecture (Padera et al, 2004).

In the early $20^{\text {th }}$ century, radiation therapy in conjunction with surgery was the only means of treating cancer, and was the predominant treatment as late as the 1960s 
(DeVita and Chu 2008). The possibility of the treatment of cancers by administration of chemicals was hypothesised as early as the late 1800s (DeVita and Chu 2008), but it was not until the mid 1900s that the broad range of chemicals that might affect the disease was narrowed. An accidental spill of sulphur mustards on troops led to the observation that bone marrow and lymph nodes were significantly depleted in those exposed (DeVita and Chu 2008). This observation led to research into the chemistry and potential therapeutic effects of mustards. Rodents were used as animal models with transplantable tumours to test for these hypothesised effects, and methods were developed to screen chemicals (DeVita and Chu 2008). Marked regression of these tumours, and subsequently tumours in human patients, were observed (DeVita and Chu 2008). Modern day cancer treatments still combine these three strategies; the actions of chemotherapeutic agents, with radiation therapy, and surgery. More recently however lead compounds used in chemotherapy have become a lot more focused and are targeted to unique characteristics of cancer cells.

\subsection{Hypoxia targeted prodrugs}

A lot of contemporary research has focused on the use of prodrugs to target unique characteristics of cancer, as a means of improving the therapeutic index of the drug administered.

Prodrugs are drugs that are administered to cells in forms that are harmless, or have relatively minimal detrimental effects, until conversion to their activate forms. Once a prodrug has become activated it is cytotoxic to its surrounding environment through various mechanisms including DNA cross-linking, chromosomal aberrations, and inhibition of DNA synthesis ultimately resulting in apoptosis. The activation of prodrugs can be induced by a number of physiological conditions, including changes in temperature, $\mathrm{pH}$, and salt concentration. Hypoxic conditions, which are uniquely characteristic of solid tumours, have also been exploited to induce the activation of prodrugs.

Hypoxia is a common feature of a large proportion of human tumours (Patterson et al, 2007). It is typically more severe in cancerous tumors than in healthy tissues, thus providing a basis for tumor selectivity (Brown and Giaccia, 1998). 
A by-product of the highly unorganised nature of the tumour vasculature network is the inadequate oxygenation of all tumour cells. This unique anatomy makes current models of cancer treatment inadequate. Hypoxia can compromise outcomes of surgery by increasing tumor metastasis (Patterson et al, 2007). Moreover, radiation therapy relies on molecular oxygen to aid in DNA damage induction via ROS (Brown and Wilson, 2004), thus due to their hypoxic nature, cancer cells are much less sensitive to the effects of radiation therapy. There is also a correlation between hypoxic regions, and the distance from the vasculature, which makes it difficult to target the origin of the tumour for circulating drugs (Dachs et al, 2009). Many drugs have been designed with the knowledge that cancer is a rapidly proliferating disease, but hypoxic regions tend to show a decreased rate of cellular growth leaving them resistant to many antiproliferative chemotherapy agents (Kennedy et al, 1997).

These problems are being overcome by a new generation of prodrugs, which are selectively activated in hypoxic cells to release or activate a toxic substrate capable of killing surrounding oxygenated tumour cells. Some useful compound groups include $\mathrm{N}$-oxides, quinones, and nitroaromatics (Denny, 2000).

The usual mechanism of action of hypoxia activated prodrugs, is that the prodrug acts as a substrate for an endogenous one electron reductase, thereby introducing an electron and forming a free radical. In oxic cells there are little to no harmful effects as this one electron reduction is part of a futile cycle; the unpaired electron is quickly transferred to molecular oxygen forming superoxide, and re-establishing the initial prodrug (Brown and Wilson 2004). However, in hypoxic cells, the cytotoxic form is able to become biologically available if the concentration of oxygen is low enough that the levels of the activated prodrug are able to accumulate without being cycled back into the original non-toxic form (Figure 1.1). In the majority of cases, this cytotoxic product undergoes more reactions to form a compound which is highly toxic to surrounding cells (Brown and Wilson, 2004). 
a Oxic cell

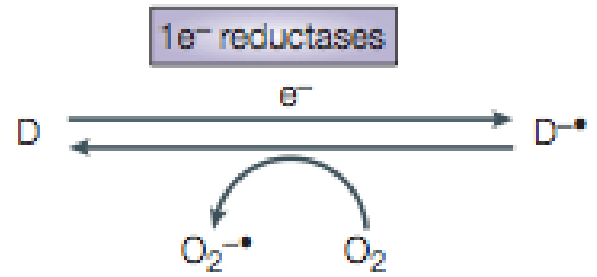

b Hypoxic cell

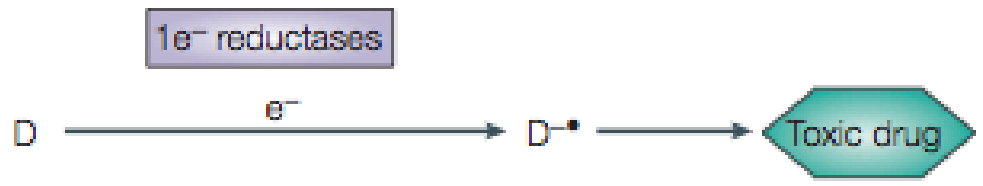

Figure 1.1 Hypoxia prodrug mechanism of activation. 1 electron reduction by endogenous enzymes; futile cycling back to original drug in oxic cell, superoxide metabolised to more toxic drug in hypoxic cell due to lack of oxygen to enter futile cycle. (Brown and Wilson, 2004)

In vivo these cytotoxic effects are masked by their inactivity towards resistant oxic cells, suggesting that to be beneficial these hypoxic selective anti tumour agents would need to be used in combination with other treatments that are effective against oxic cells, like radiotherapy (Bremmer, 1993).

\subsubsection{Nitroaromatic hypoxia activated prodrugs}

Recently, great promise has been exhibited by nitroaromatic hypoxia targeting prodrugs, which are molecules of direct relevance to this research. In hypoxic human cells, these prodrugs are reduced in a stepwise manner of one electron at a time, up to six, until the end metabolite is generated (Denny, 2000). In oxic cells however, the nitro radical anion produced in the first step of this reaction pathway can be scavenged by molecular oxygen, leaving the prodrug inactive under non-hypoxic conditions (Denny, 2000) (Figure 1.2).

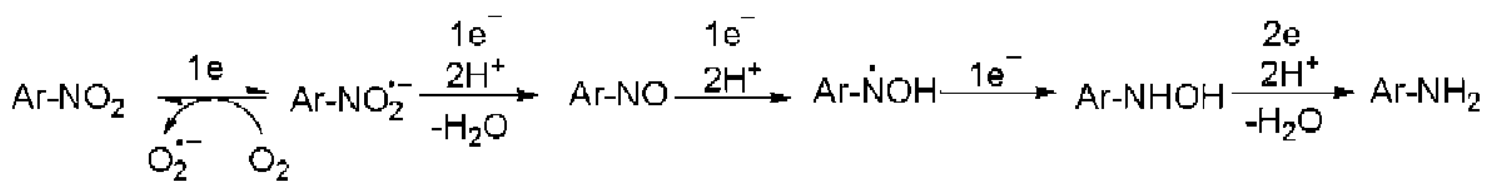

Figure 1.2 Hypoxia activation of nitroaromatic prodrug. Stepwise reduction reactions, 1 electron at a time, to generate a cytotoxic metabolite. The first reduction reaction can enter a futile cycle in oxic cells. (Chen and $\mathrm{Hu} 2009$ ) 
Nitroaromatic compounds for use in anti-cancer therapy were first developed from early nitroimidazole based radiosensitisers, which are selectively metabolised under hypoxic conditions (Chen and $\mathrm{Hu} 2009$ ). These radiosensitisers were only weakly cytotoxic, but newer generation drugs based on the original structures were developed to produce a higher toxic effect in the cell (Chen and $\mathrm{Hu} 2009$ ).

The first of the nitroaromatics discovered that was able to be reduced under hypoxic conditions was 1-(2-nitro-1-imidazolyl-1)3-methoxypropanol (misonidazole). As misonidazole was shown to improve radiotherapy in vivo, a number of other nitroimidazole-based derivatives were then developed to give greater cytotoxicity and improved hypoxia selectivity (Chen and $\mathrm{Hu} 2009$ ). One of these nitroimidazolebased derivatives is 1-(2-nitro-1-imidazolyl)-3-(1-aziridino)-2-propanol (RSU1069). RSU1069 is a 2-nitroimidazole with an aziridine moiety in the N1 side chain, enabling bifunctional alkylation upon reduction (Chen and $\mathrm{Hu}$ 2009, Deacon et al 1986) (figure 1.3). In vitro and in vivo studies have both shown that RSU1069 is more a more efficient radiosensitiser than misonidazole (Deacon et al 1986) (figure $1.3)$.

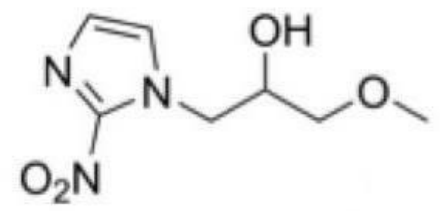

Misonidazole

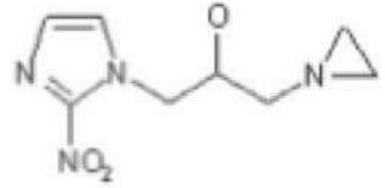

RSU1069

Figure 1.3 Structures of nitroaromatic anti-cancer compounds. Misonidazole, and RSU1069.

The dinitrobenzamide 5-aziridin-1-yl-2-4- dinitrobenzamide (CB1954) is also activated under hypoxic conditions by the endogenous 2 electron reductase, $\mathrm{NAD}(\mathrm{P}) \mathrm{H}$ oxidoreductase (DT diaphorase), although, due to the reduction of 2 electrons, the oxygen reversible intermediate is bypassed leading to poor hypoxic selectivity (Denny, 2000). This has initiated the development of a range of hypoxiasensitive derivatives, in particular the dinitrobenzamide mustards (DNBMs). This class of compound was originally developed following investigations into the enhancement of toxicity and hypoxia selectivity of nitrophenyl prodrugs, through the addition of a nitrogen mustard functional group (Denny and Wilson, 1986). The 
alkylating properties of the dormant mustard are restored following nitroreduction as shown in figure 1.4.

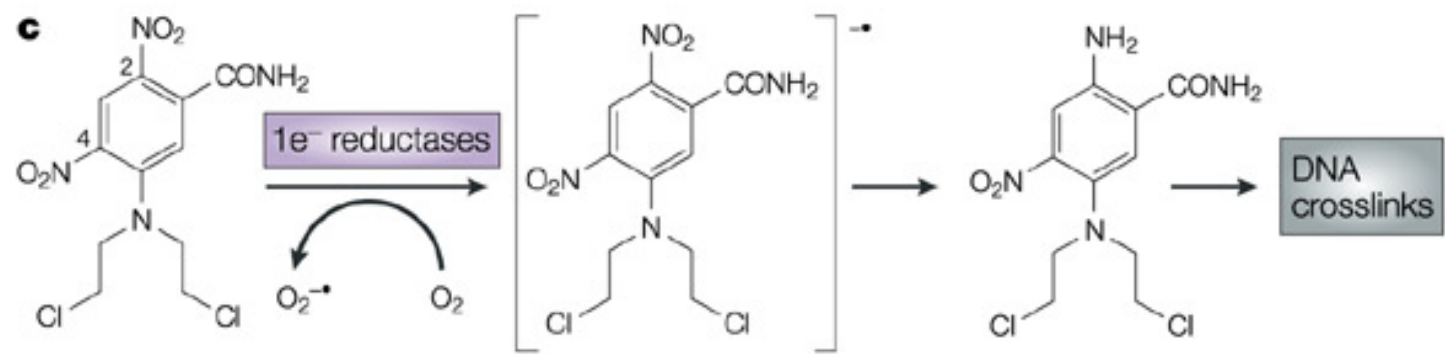

Figure 1.4 Hypoxia activated nitroaromatic prodrug. Reduction occurs through one-electron reductases via an oxygen-sensitive free radical intermediate to a cytotoxic metabolite which induces DNA crosslinking causing apoptosis (Brown and Wilson 2004).

\subsection{GDEPT}

Previous research into the field of enzyme and hypoxia activated prodrugs has paved the way for GDEPT, which builds on this current knowledge and provides new cutting edge technology. GDEPT has the potential to selectively eradicate tumour cells, while at the same time sparing normal tissue from damage (Greco and Dachs, 2001). It is based on the delivery of a gene that encodes an enzyme which is nontoxic in itself, but which is able to convert a prodrug into a potent cytotoxin (Greco and Dachs, 2001), leaving the transfected cells sensitised to the administered prodrug. GDEPT aims to bypass the molecular restrictions of current cancer therapies, utilising the ability of exogenous enzymes (often, but not exclusively, of bacterial origin) to activate prodrugs. This would leave targeting hypoxia unnecessary, by targeting specific molecules on the outside of tumour cells for gene delivery (Dachs et al, 2009), and activation of the prodrug induced by the delivered gene (figure 1.5).

A major hurdle to overcome in cancer GDEPT is the delivery of the genes to a sufficient number of tumour cells to induce regression (Rainov, 2000). Despite active research, gene transfer efficiencies in the clinic have appeared to be at a $10 \%$ maximum (Dachs et al, 2009). This limitation can however, be addressed by employing prodrugs with an efficient bystander effect; that is which induce the death of neighbouring non-transgenic cells as well as the transgenic cells that activate them (Dachs et al, 2009). A great advantage of nitroaromatic prodrugs in this context is 
they can be chemically engineered to have an optimal bystander effect to maximise tumour cell kill, without impairing activation by their nitroreductase partner (Dr Adam Patterson, personal communication).

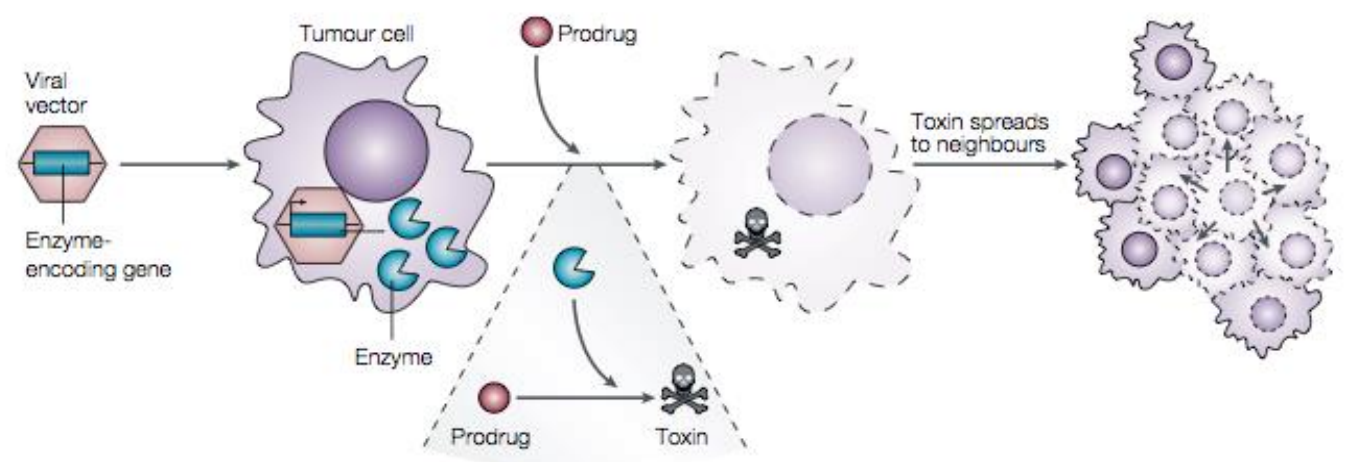

Figure 1.5 GDEPT Overview. Vector uptake into the cell resulting in enzyme expression in a small percentage of target cells. The bystander effect is achieved by subsequent genotoxic effect to transgenic and neighbouring cells (McCormick, 2001)

\subsubsection{E. coli nitroreductase (NfsB) and 5-aziridin-1-yl-2-4- dinitrobenzamide (CB1954)}

An isolated nitroreductase gene from $E$. coli, $n f s B$, expresses the NfsB_Ec nitroreductase enzyme that has been shown to activate the prototypical nitroaromatic prodrug, 5-(aziridin-1-yl)-2,4-dinitrobenzamide (CB1954) to its toxic form.

$\mathrm{NfsB}$ is known to reduce a wide range of nitroaromatics, and was initially identified as a target for antibiotics (Christofferson, 2009). Its mechanism of action is dependent on FMN; NAD(P)H binds to the enzyme, and donates two electrons to the FMN cofactor, then $\mathrm{NAD}(\mathrm{P})+$ is subsequently released, enabling the substrate to bind the active site, and be reduced itself (Christofferson, 2009, Parkinson, 2000). The simultaneous 2-electron reduction of the nitro group to an electron donating hydroxylamine renders the reaction oxygen independent (Christofferson, 2009). The cytotoxin then induces DNA crosslinks which ultimately result in apoptosis. A major advantage of this prodrug-enzyme combination is that cell death isn't dependent on 
active cellular replication, apoptosis can in occur in cells in arrested cycle too (Christofferson, 2009).

CB1954 isn't a natural substrate for NfsB_Ec, and has low affinity for the enzyme resulting in a low yield of cytotoxic drug (Christofferson, 2009). Regardless, the NfsB_Ec-CB1954 combination has has been evaluated in Phase I/II trials in combination and further nitroreductase / prodrug combinations are continually being explored.

There is vast potential for the discovery of other nitroreductases that may confer higher activity than NfsB_Ec, enabling more efficient prodrug activation.

A number of novel candidates, from $E$. coli as well as other bacteria, have been identified as having nitroaromatic reducing capabilities. Many of these have not yet been screened for activity with anti-tumour prodrugs. However, some that have are a series of enzymes related to the Bacillus amyloliquefaciens nitroreductase $y w r O$, which shares amino acid similarities to mammalian NQO1 (Theys et al., 2006). Another more recently investigated enzyme family that offers great promise are the orthologues of a second nitroreductase of $E$. coli, $n f_{s} A$, which has also been studied in combination with the CB1954 prodrug (Vass et al., 2009; Prosser et al., 2010a).

There is also the potential for the use of nitroreductases for the activation of other potentially beneficial substrates, such as nitro-quenched nil bystander antibiotics, like metronidazole, that can eliminate vectors post-therapy; or nitro-quenched fluorophores, with imaging potential. Both of these aspects will be discussed later in this introduction.

Based on this prior work, as well as the discovery of other enzymes with nitroreductase potential, researchers in the Ackerley lab have generated a large collection of nitroreductase candidates and have screened this for activity with various nitroaromatic prodrugs (Prosser et al, 2010a,b; Prosser et al 2013). 


\subsection{The 58-membered nitroreductase library}

The two main families and best studied of the nitroreductase enzymes are $n f s A$ and $n f s B$. They are named for nitrofuran sensitivity, having originally been discovered on the basis of mutations in the $E$. coli genes being found to confer resistance to the antibiotic nitrofuran (Bryant et al., 1981; Whiteway et al., 1998). The characteristics that distinguish these two major families are that the $n f_{s} A$ family (type I) nitroreductases which are dependent on NADPH to reduce their substrates, and the $n f s B$ family (type II) can use either NADH or NADPH for reduction (Zenno et al., 1996)

$n f s A$ is induced via oxidative stress as part of the soxRS regulon (Paterson, 2002). Its homolog in Bacillus subtilis has also been shown to be induced under oxidative stress, as well as heat shock (Streker et al., 2005). There are 20 homologs of E. coli nfsA within the nitroreductase library (Table 1.1).

$n f s B$ is a monomeric flavin mononucleotide (FMN) containing flavoprotein (Zenno et al., 1996) The $n f s B$ family is capable of utilising both NADH and NADPH as cofactors to efficiently reduce a number of nitro aromatic substrates (Zenno et al., 1996). There are 12 homologs of E. coli $n f s B$ in the core library (Table 1.1).

The remaining 26 nitroreductase enzymes come from 11 different families; $w r b A$, yieF, azoR, mdaB, ywrO, ydjA, nemA, NQO1, kefF, ycaK, and ycdI (Prosser et al., 2013). Verified nitroreductase activity has previously been observed for one or more members of the $y i e F, a z o R, m d a B, y w r O$, nemA and NQO1 families, but not for the remainder (Theys et al, 2006; Barak et al., 2006, Prosser et al., 2013).

Table 1.1 Complete 58 nitroreductase library

\begin{tabular}{|r|l|l|l|l|}
\hline$\#$ & Gene & Organism & Family & $\%$ ID/Sim \\
\hline 1 & NfsA_Ec & Escherichia coli & NfsA & $100 / 100$ \\
\hline 2 & NfsA_St & Salmonella enterica & NfsA & $87 / 95$ \\
\hline 3 & NfsA_Ck & Citrobacter koseri & NfsA & $86 / 92$ \\
\hline 4 & NfsA_Kp & Klebsiella pneumoniae & NfsA & $83 / 92$ \\
\hline 5 & NfsA_Es & Enterobacter sakazakii & NfsA & $82 / 94$ \\
\hline 6 & NfsA_Ecaro & Erwinia carotovara & NfsA & $65 / 82$ \\
\hline 7 & NfsA_Vf & Vibrio fischeri & NfsA & $52 / 68$ \\
\hline
\end{tabular}




\begin{tabular}{|c|c|c|c|c|}
\hline 8 & NfsA_Vv & Vibrio vulnificus & NfsA & $51 / 65$ \\
\hline 9 & Frp1_Vh & Vibrio harveyi & NfsA & $51 / 65$ \\
\hline 10 & Frp2_Vh & Vibrio harveyi & NfsA & $51 / 65$ \\
\hline 11 & NfrA_Bs & Bacillus subtilis & NfsA & $39 / 62$ \\
\hline 12 & NfsA_Lw & Listeria welshimeri & NfsA & $41 / 60$ \\
\hline 13 & NfsA_Li & Listeria innocua & NfsA & $39 / 60$ \\
\hline 14 & NfsA_Bco & Bacillus coagulans & NfsA & $38 / 60$ \\
\hline 15 & NfsA_Np & Nostoc punctiforme & NfsA & $38 / 59$ \\
\hline 16 & NfsA_Bth & Bacillus thuringiensis & NfsA & $35 / 58$ \\
\hline 17 & NfsA_Lsak & Lactobacillus sakei & NfsA & $40 / 57$ \\
\hline 18 & NfsA_Pp & Pseudomonas putida & NfsA & $35 / 57$ \\
\hline 19 & YcnD_Bs & Bacillus subtilis & NfsA & $35 / 56$ \\
\hline 20 & NfsA_Ms & Mycobacterium smegmatis & NfsA & $36 / 54$ \\
\hline 21 & NfsB_Ec & Escherichia coli & NfsB & $100 / 100$ \\
\hline 22 & NfsB_Ck & Citrobacter koseri & $\mathrm{NfsB}$ & $88 / 95$ \\
\hline 23 & NfsB_St & Salmonella enterica & $\mathrm{NfsB}$ & $88 / 91$ \\
\hline 24 & NfsB_Kp & Klebsiella pneumoniae & NfsB & $84 / 93$ \\
\hline 25 & NfsB_Vv & Vibrio vulnificus & $\mathrm{NfsB}$ & $61 / 75$ \\
\hline 26 & NfsB_Pp & Pseudomonas putida & NfsB & $52 / 71$ \\
\hline 27 & NfsB_Es & Enterobacter sakazakii & NfsB & $46 / 66$ \\
\hline 28 & FraseI_Vf & Vibrio fischeri & NfsB & $34 / 53$ \\
\hline 29 & NfsB_Vh & Vibrio harveyi & NfsB & $30 / 48$ \\
\hline 30 & YfkO_Bs & Bacillus subtilis & NfsB & $29 / 46$ \\
\hline 31 & YdgI_Bs & Bacillus subtilis & $\mathrm{NfsB}$ & $25 / 47$ \\
\hline 32 & NfsB_Pa & Pseudomonas aeruginosa & NfsB & $24 / 42$ \\
\hline 33 & AzoR_Ec & Escherichia coli & AzoR & $100 / 100$ \\
\hline 34 & AzoR_St & Salmonella enterica & AzoR & $87 / 95$ \\
\hline 35 & AzoR_Vv & Vibrio vulnificus & AzoR & $56 / 73$ \\
\hline 36 & AzoR_Pp & Pseudomonas putida & AzoR & $48 / 66$ \\
\hline 37 & NemA_Ec & Escherichia coli & NemA & $100 / 100$ \\
\hline 38 & NemA_St & Salmonella enterica & NemA & $92 / 96$ \\
\hline 39 & NemA_Kp & Klebsiella pneumoniae & NemA & $86 / 92$ \\
\hline 40 & NemA_Vv & Vibrio vulnificus & NemA & $47 / 65$ \\
\hline 41 & YwrO_Bs & Bacillus subtilis & YwrO & $70 / 82$ \\
\hline 42 & YwrO_Li & Listeria innocua & YwrO & $42 / 57$ \\
\hline 43 & YwrO_Vf & Vibrio fischeri & YwrO & $47 / 62$ \\
\hline 44 & YieF_Ec & Escherichia coli & YieF & $100 / 100$ \\
\hline 45 & YieF_Pa & Pseudomonas aeruginosa & YieF & $45 / 60$ \\
\hline 46 & MdaB_Ec & Escherichia coli & $\mathrm{MdaB}$ & $100 / 100$ \\
\hline 47 & MdaB_Ps & Pseudomonas syringae & MdaB & $60 / 74$ \\
\hline 48 & WrbA_Ec & Escherichia coli & WrbA & $100 / 100$ \\
\hline 49 & WrbA_Ps & Pseudomonas syringae & WrbA & $39 / 55$ \\
\hline 50 & YdjA_Ec & Escherichia coli & YdjA & $100 / 100$ \\
\hline 51 & YdjA_Kp & Klebsiella pneumoniae & YdjA & $79 / 89$ \\
\hline 52 & NQO1_Pp & Pseudomonas putida & NQO1 & $37 / 56$ \\
\hline
\end{tabular}




\begin{tabular}{|r|l|l|l|l|}
53 & NQO1_Pa & Pseudomonas aeruginosa & NQO1 & $41 / 54$ \\
\hline 54 & YcdI_Ec & Escherichia coli & YcdI & $100 / 100$ \\
\hline 55 & YcdI_Kp & Klebsiella pneumoniae & YcdI & $76 / 85$ \\
\hline 56 & YcaK_Ec & Escherichia coli & YcaK & $100 / 100$ \\
\hline 57 & YcaK_Pa & Pseudomonas aeruginosa & YcaK & $27 / 48$ \\
\hline 58 & KefF_Ec & Escherichia coli & KefF & $100 / 100$ \\
\hline
\end{tabular}

* Frp1_Vh and Frp2_Vh are versions of Frp cloned from slightly different strains of Vibrio harveyi

\subsection{Nil bystander antibiotics}

The ability to safely eliminate the vector from patients is an essential feature for a complete GDEPT system, as pathogenic bacterium - such as Clostridium - are commonly used vectors in solid tumour targeting. Due to their pathogenic nature, the FDA require that there be a way to eliminate these vectors from the patient post treatment.

The Ackerley lab been able to show that many nitroreductases that activate anticancer prodrugs have the additional ability to reduce a range of nil bystander nitroaromatic antibiotics, and that this may be able to provide a potential safety mechanism for eliminating biological vectors from patients post-therapy or in case of an adverse event.

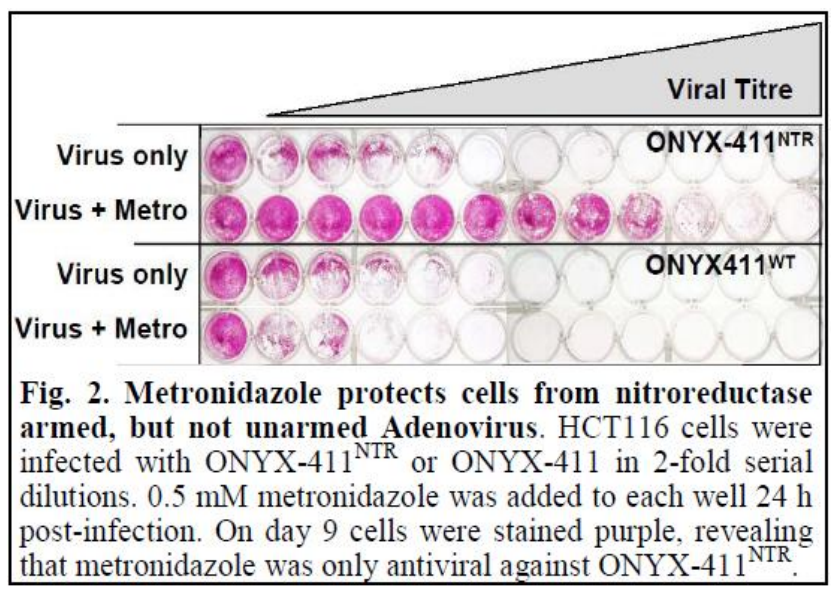

Figure 1.6 Dr Adam Patterson, University of Auckland, unpublished work. Metronidazole is capable of suppressing the replication of the ONYX-411 adenovirus while carrying a nitroreductase, but not the wildtype ONYX-411 adenovirus. 
Dr Patterson's lab in Auckland have shown that two of the 16 candidate nil bystander antibiotics that were tested in this thesis, metronidazole and tinidazole, are widely activated by orthologues of $\mathrm{NfsA}(\mathrm{Ec})$ and $\mathrm{NfsB}(\mathrm{Ec})$, and that this activity has potential for eliminating gene therapy vectors from the host. It is intuitive how bacterial vectors might be sensitive to antibiotics; indeed, metronidazole is already the antibiotic of choice for eliminating Clostridium from patients (Basu, 2010). However, Dr Patterson's group have also been able to show that metronidazole has the ability to suppress the replication of the ONYX-411 adenovirus when labelled with a nitroreductase, but not the wildtype ONYX-411 adenovirus (Figure. 1.6). This observation that nitroreductases in conjunction with nil-bystander antibiotics are also able to be utilised to eliminate viral vectors was a key factor motivating this research, suggesting that we might be able to interrogate the 58 nitroreductase core library to recover multi-functional nitroreductases for use in an all encompassing GDEPT system.

\subsection{Masked fluorophores}

A library of nitro-quenched masked fluorophores has also been developed by the Auckland Cancer Society Research Centre. These compounds don't show fluorescence until they are activated, which can be achieved through the reduction mechanisms of nitroreductases. There are two distinct categories of masked fluorophore; nitro-switch or bioreductive trigger (BRT) as depicted in figure 1.7 (Horvat 2012).<smiles>CN(C)CCNC(=O)c1cc([N+](=O)[O-])cc2c(=O)c3ccccc3[nH]c12</smiles>

FSL76 Nitroswitch quenched fluorophore<smiles></smiles>

FSL178

Bioreductive trigger quenched fluorophore

Figure 1.7 FSL76 and FSL178. Examples of the two different kinds of quenched fluorophore; Nitroswitch and bioreductive trigger. 
Nitro-switch fluorophores are reduced at the nitro group, causing a change in electronic charge that directly activates fluorescence (Horvat 2012).

BRT molecules are made up of three distinct parts; a trigger, a linker and an effector (figure 1.8). The trigger is the substrate for the activating enzyme, the linker is broken during this metabolic process, resulting in either the effector becoming activated or released (Siim, et al., 1997). In the case of the masked fluorophores, release of the effector moiety generates a fluorescent signal.

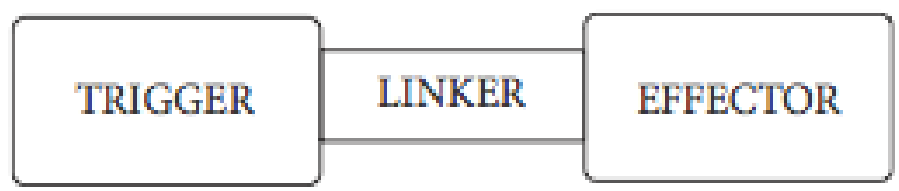

Figure 1.8 Bioreductive trigger molecule components. Make up and position of fragments in a bioreductive trigger molecule (Siim, et al., 1997).

Currently in the Ackerley lab, both types of masked fluorophores are being utilised in the discovery and evaluation of novel nitroreductases.

\subsection{Regenerative and developmental biological studies}

Developmental biology looks to understand which cellular mechanisms are involved in which processes of development. The study of cell specific regeneration enables insights into the differentiation of specific cell types and regenerative potential of individual stem cell populations. Conditional targeted ablation is a powerful tool that can be used to study precise stages of development, and the ability to temporally and spatially control tissue damage and remove a specific cell population has wide applications for regeneration studies. Previous technology for use in regenerative studies has not been regulated on such a targeted scale, with studies focusing on more complex scenarios, such as whole limb regeneration, which involves various cell and tissue types. The ability to utilise nitroreductases in temporally and spatially targeted cell ablation will be beneficial to the study of many degenerative diseases that stem from the loss of individual cell types (Kaya et al., 2012) 
A wide range of genes have important roles during both embryogenesis and adulthood, knockout of these genes can cause a lethal phenotype in the embryo, making it difficult to generate animal models to study the function of the gene in later stages of development, or its role in adulthood (Davison et al., 2007). Another obstacle is that many phenotypes caused by gene knockout may be due to systemic effects in the whole organism rather than a specific tissue, making them hard to analyse (Davison et al., 2007). Due to this, many studies have attempted to develop genetic switches to allow the silencing of genes temporally and spatially. Essential factors for such a targeted cell ablation system in developmental and regenerative studies are the ability of the mechanism to not interfere with other genetic components, and the reversibility of the silencing. Early attempts to develop this type of switch employed the use of single transgenes with promoters that could be induced by heavy metals, heat shock, interferon or steroids. Pleiotropic effects and high basal activity in the absence of induction rendered these systems somewhat ineffective. Instead, binary transgenic systems, in which gene expression is controlled by the interaction of two components - an effector transgene, the product of which acts on a target transgene - are often used for these studies, but require complex genetic manipulations to achieve. Physical surgery and laser-mediated ablation methods are also commonplace, although they are labour-intensive, time-consuming, and not as reproducible as a genetic approach, which limits their use in large scale screening experiments.

All of these processes have been developed to conditionally target ablation, but none have been able to meet four key criteria simultaneously; to be spatially controllable and strictly confined to the target cell population, to be temporally inducible, to be germline transmissible and, to be reversible (Curudo et al., 2007). The implementation of a fluorescent protein/ nitroreductase fusion protein system to ablate target cell populations could vastly improve this technology.

A handful of studies, primarily using Xenopus and zebrafish as model organisms, have found success in using nitroreductase genes to promote targeted cell ablation in distinct cell populations for application in these fields. 


\subsubsection{Case studies utilising nitroreductase ablation technology}

A handful of studies have shown that the fluorescent protein/ nitroreductase fusion technique is capable of ablating target cell populations in a way that is spatially controllable and strictly confined to the target cell population, temporally inducible, germline transmissible and reversible (Curado and Stainier 2008)

Many of these initial studies have targeted the ablation and subsequent regeneration of islet $\beta$ cells in an effort to study the inducibility and endogenous regeneration of $\beta$ cells in type 1 diabetics to recover glucose homeostasis (Curado et al., 2007).

One of these studies (Curado et al., 2007) achieved this via a CFP/ nitroreductase fusion protein linked to the insulin promoter. The promoter drives the expression of CFP and a nitroreductase (NfsB_Ec). The expression of the CFP allows reporting on the cell population, as fluorescence is only visible in the cell population under the control of the promoter which is also expressing NfsB_Ec. The addition of a nil bystander antibiotic, metronidazole, to the system destroys the cell population, as metronidazole is reduced to its cytotoxic form in the presence of NfsB_Ec. Figure 1.9 from Curado et al (2007) shows the promoter construct used in this study, and also visualisation of the zebrafish before (B) and after (C) the addition of metronidazole to the organism's environment. 

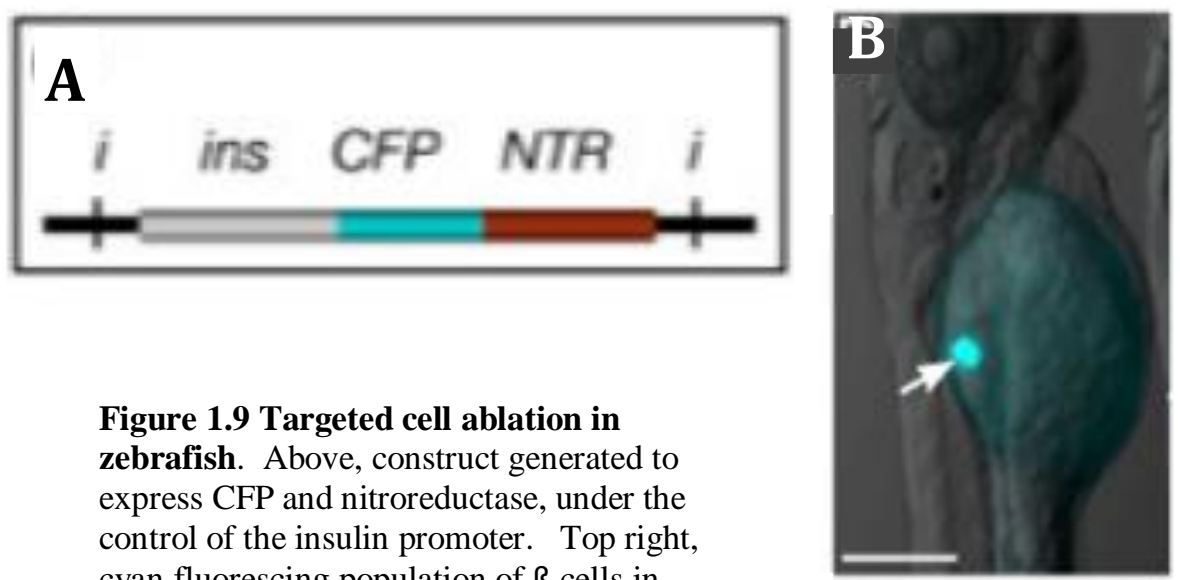

Figure 1.9 Targeted cell ablation in zebrafish. Above, construct generated to express CFP and nitroreductase, under the control of the insulin promoter. Top right, cyan fluorescing population of $ß$ cells in transgenic zebrafish. Bottom right, transgenic zebrafish after metronidazole treatment, ß cell population is longer fluorescing cyan.

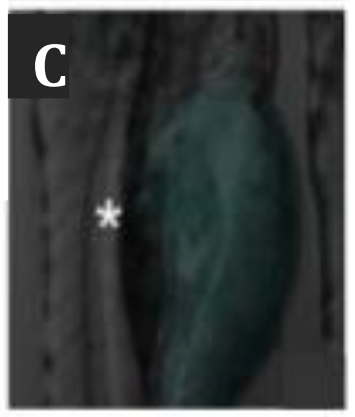

Kaya et al, 2012 explored demyelination and remyelination in Xenopus utilising a GFP: nitroreductase system under the control of the myelin basic protein, which codes for mature oligodendrocytes. Oligodendrocytes create myelin sheaths, which form a layer around the axon of a neuron that is essential for the proper functioning of the nervous system. Demyelination is implicated in many degenerative diseases. This study used live imaging to show that the removal of metronidazole from the organism's environment resulted in the complete regeneration of the target cell population (Figure 1.10). 

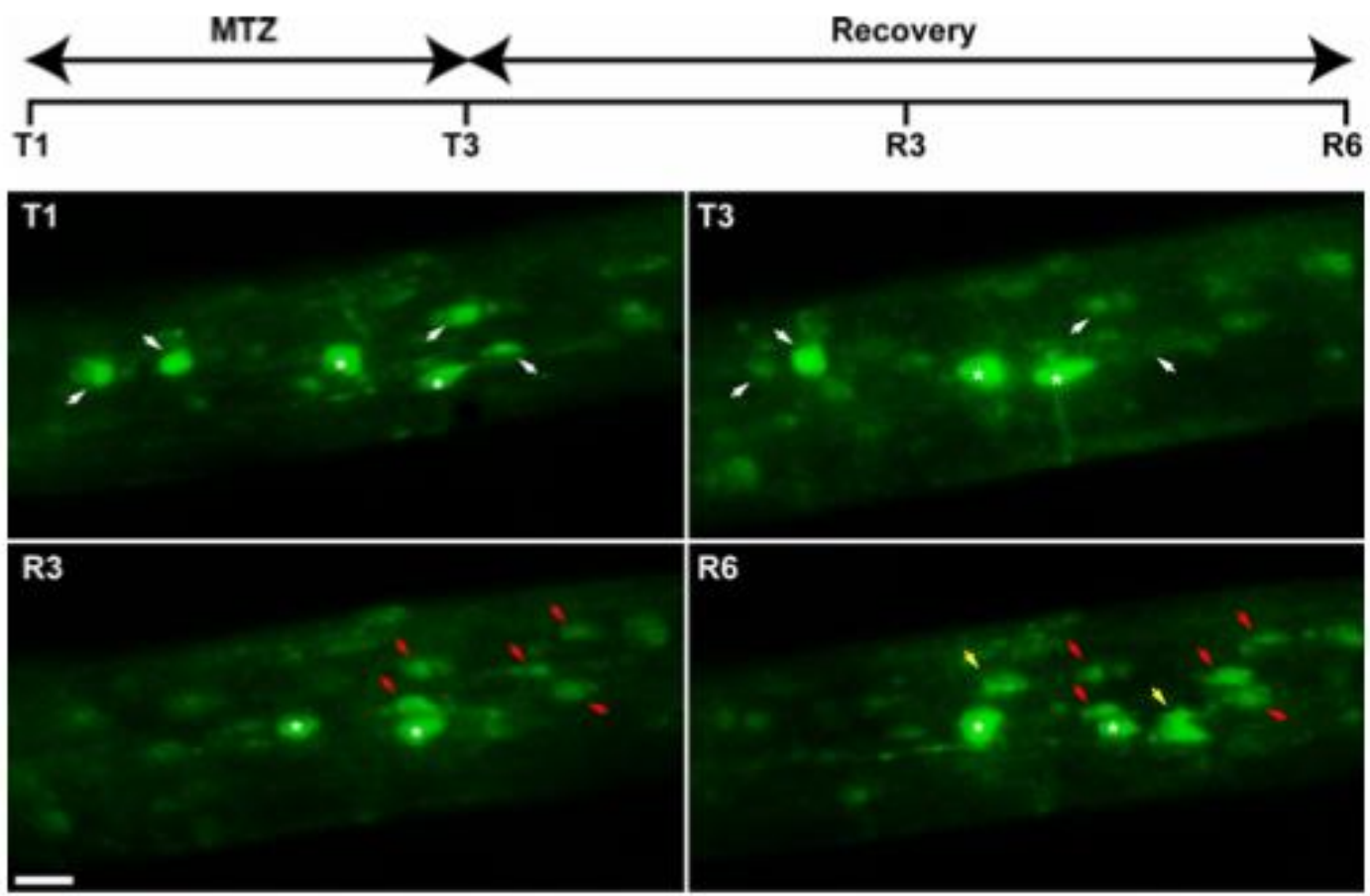

Figure 1.10 Live imaging of oligodendrocyte depletion and reappearance following metronidazole treatment and cessation. Successive observation of the optic nerve of the same transgenic tadpole by two-photon microscopy. Transgenic tadpoles were treated for $3 \mathrm{~d}$ with metronidazole $(10 \mathrm{mM})(\mathrm{T} 1, \mathrm{~T} 3)$, then returned to normal water for $6 \mathrm{~d}$. Observations were before treatment (T1), after $3 \mathrm{~d}$ in the presence of metronidazole (T3) and during recovery at $3 \mathrm{~d}$ and $6 \mathrm{~d}$ in normal water (R3, R6). (Kaya et al., 2012)

\subsubsection{Limitations of the NfsB_Ec/ metronidazole system}

One clear limitation of this system, is that the nitroreductase currently being used in these studies, NfsB_Ec, is insufficiently active with metronidazole to give clean targeted cell ablation without high background toxicity. Although not mentioned in the papers that employ this ablation system, the toxicity of metronidazole to nontransgenic zebrafish (at the doses required for complete ablation of NfsB_Ec expressing cells) is very high, such that relatively few fish from the original cohort are able to survive a multi-day experiment (Dr J.S. Mumm, Georgia Regents University, personal communication).

\subsubsection{Combining this technology with masked fluorophores}

Work by Singleton et al (2007) was the first to show that, as well as being capable of activating a wide range of drugs and antibiotics, nitroreductases are also capable of directly activating fluorescent reporter molecules. Although fluorescent proteins used 
in the fluorescent protein/ nitroreductase system are successful reporters, this realisation illustrates that fluorescent protein tags are not in fact necessary for such studies. A single nitroreductase has the potential to activate both a nil bystander antibiotic, and a masked fluorophore simultaneously.

\subsubsection{Dual reporter/ ablation system exploiting the characteristics of nitroreductases}

Exploiting the ability of nitroreductases to activate both nil bystander antibiotics and masked fluorophores simultaneously, and building on the base technology developed by Curado et al, we propose a two-way opposing activation system, in which one nitroreductase will be able to activate a specific antibiotic and fluorophore, while being inactive with a different antibiotic and fluorophore which is able to be activated by another nitroreductase (figure 1.11). This will allow the temporal and spatial reporting and targeted ablation of two distinct cell populations at once, giving efficient imaging of the two different populations of cells simultaneously, and the ablation of one or even both at specific stages of development as desired.

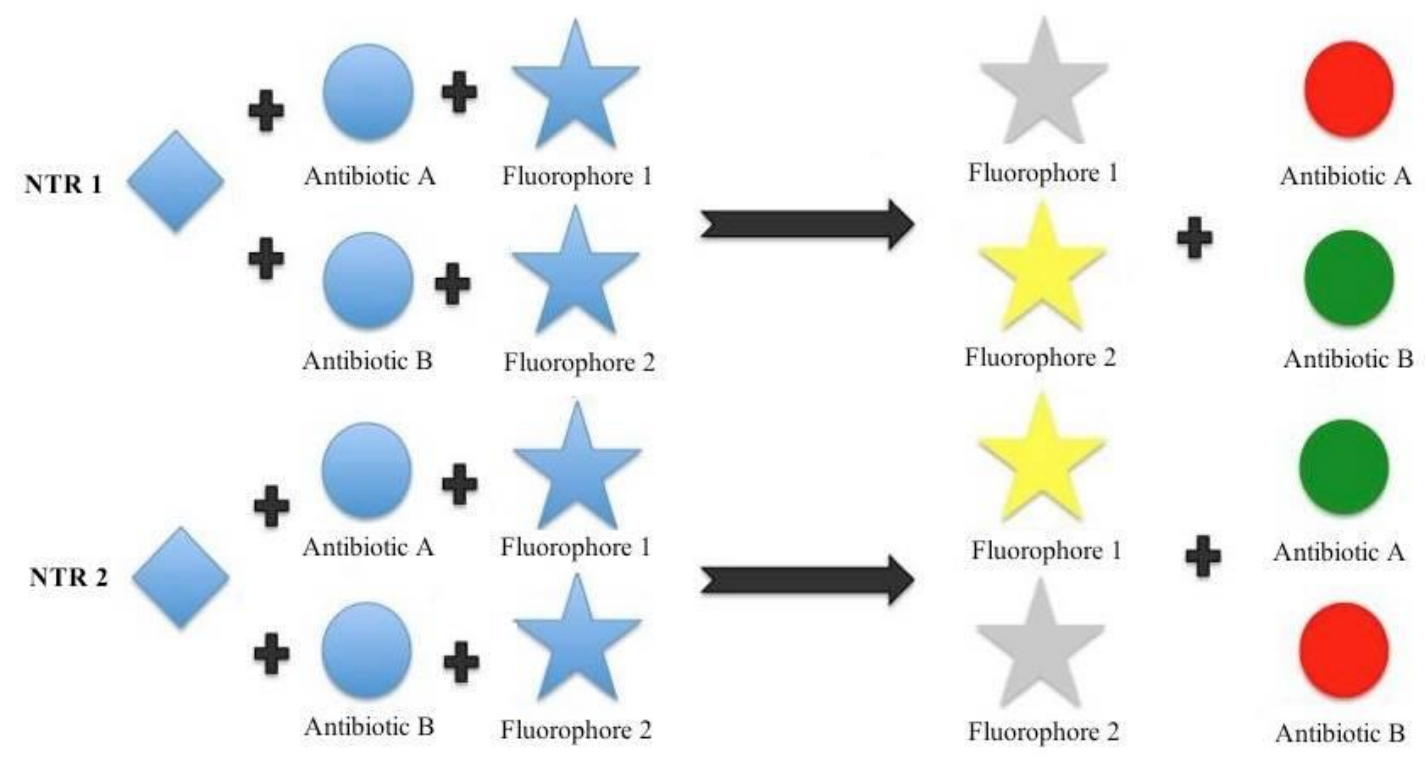

Figure 1.11 Nitroreductases with opposing specificities for both masked fluorophores and nilbystander antibiotics. Nitroreductase 1 has activity with antibiotic B and fluorophore 2, but not antibiotic A or fluorophore1, while nitroreductase 2 has the opposing activation profile; activity with antibiotic A and fluorophores 1, but not antibiotic B or masked fluorophore 2. 


\subsection{Aims}

This research project has various evolving aims. Firstly, to identify a nitroreductase which has a better activation profile with metronidazole than NfsB_Ec. Following this, the full nitroreductase library will be screened with a number of nil bystander antibiotic candidates for the identification of other nitroreductase/nil bystander antibiotic combinations with potential applications in GDEPT and targeted cell ablation. Building on these initial ideas, a reporter system that eliminates the need for a bicistronic protein (currently employed in developmental and regenerative biological studies) utilising a single nitroreductase capable of activating both a masked fluorophore and a nil bystander antibiotic will be explored. The final aim is to bring all of these ideas together in a multiplex scenario, where nitroreductases with opposing activation profiles will each be capable of activating one masked fluorophore/ nil bystander antibiotic combination. This would enable spatial and temporal reporting on cell populations, and the ability to induce targeted cell death, on either one or more of these cell populations simultaneously or subsequently.

Initial screening of the 58 nitroreductase core library with metronidazole identified nitroreductases with a higher efficacy with metronidazole than $\mathrm{NfsB}$ _Ec that could be utilised in developmental and regenerative biology studies. Screening of the 58 nitroreductase core library against 16 chosen nil bystander antibiotics enabled identification of which nitroreductases have the highest activity a given nil bystander antibiotic; results that could have applications both in GDEPT, and developmental and regenerative biological studies. This screening also allowed the identification of nitroreductase / nil bystander antibiotic pairs for use in a dual targeted cell ablation system. The initial screening was undertaken in bacterial cells, with promising nitroreductases then being transfected into human cell lines for confirmation of activity in mammalian cells.

During the initial screening process, it was noticed that one of the nil bystander antibiotics that was being tested, niclosamide, acted in a completely different manner to all other nil bystander antibiotics. Instead of causing the nitroreductase it was active with to cause cell death, it appeared to have a protective effect on the cell, as cells expressing an empty plasmid had complete cell death. We proposed that this be 
used as a method to screen large number of nitroreductases as a high throughput method of novel nitroreductase discovery. This will be discussed in chapter 3 .

The second results chapter explored the ability of nitroreductases to activate a range of masked fluorophores developed by Auckland Cancer Society Research Centre available in the lab. Nitroreductases and masked fluorophores with opposing activation profiles were identified and combined with nitroreductases and nil bystander antibiotics for the development of a dual nitroreductase combined reporter/ ablation system. Again, these experiments were initially carried out in bacterial cells, with the best candidates for this system carried over for testing in human cell lines.

Although bacterial systems are extraordinarily useful for screening purposes, some nitroreductases have been shown to be unstable when transfected into human cell lines, and not behave in the same manner as observed at the bacterial level. It was due to this that it was extremely important to do testing in human cell lines as the proposed technologies would be able to be used in a mammal environment; both for effectively eliminating a vector from a patient post GDEPT therapy, and for use in developmental and regenerative biological studies. 


\section{Materials and Methods}

\subsection{Chemicals}

\subsubsection{Nil bystander antibiotics}

Stock solutions of all nil bystander candidate compounds (Table 2.1) were made to $200 \mathrm{mM}$ in anhydrous dimethyl sulphoxide (Sigma-Aldrich, St. Louis, MO,USA) and stored at $-80^{\circ} \mathrm{C}$. The only exception to this was Niclosamide, which was made to a stock solution concentration of $5 \mathrm{mM}$. All nil bystander candidates were purchased from Sigma-Aldrich (Sigma-Aldrich, St. Louis, MO, USA) with the exception of EF5, Misonidazole and F-Misonidazole (generous gifts from Dr Jeff Smaill, ACSRC) and Nifurtimox (a generous gift from Bayer Healthcare Mo, NJ, U.S).

Table 2.1 Nil bystander antibiotics

\begin{tabular}{|c|c|}
\hline Nil Bystander Antibiotic & Structure \\
\hline 4-NI & \\
\hline B-Methyl-NI &
\end{tabular}




Fimetridazole




Nifurtimox

\subsubsection{Masked fluorophores}

The masked fluorophores used in this study were developed by Dr Jeff Smaill from an existing collection of chemicals that had been synthesized by various researchers at the ASCRC over the past fifty years. Initial work carried out by Dr Horvat (Horvat, 2012) identified NTR activated fluorophores that were further evaluated in this study. 
These are shown in Table 2.2. Compound stock solutions $(10 \mathrm{mM})$ were prepared in anhydrous dimethyl sulphoxide (Sigma-Aldrich, St. Louis, MO, USA) and stored at -80 ${ }^{\circ} \mathrm{C}$.

\section{Table 2.2 Masked fluorophores}

All masked fluorophores that were used in this study. The colour of the left hand column denotes the fluorescence emission of the masked fluorophore once it has been activated.

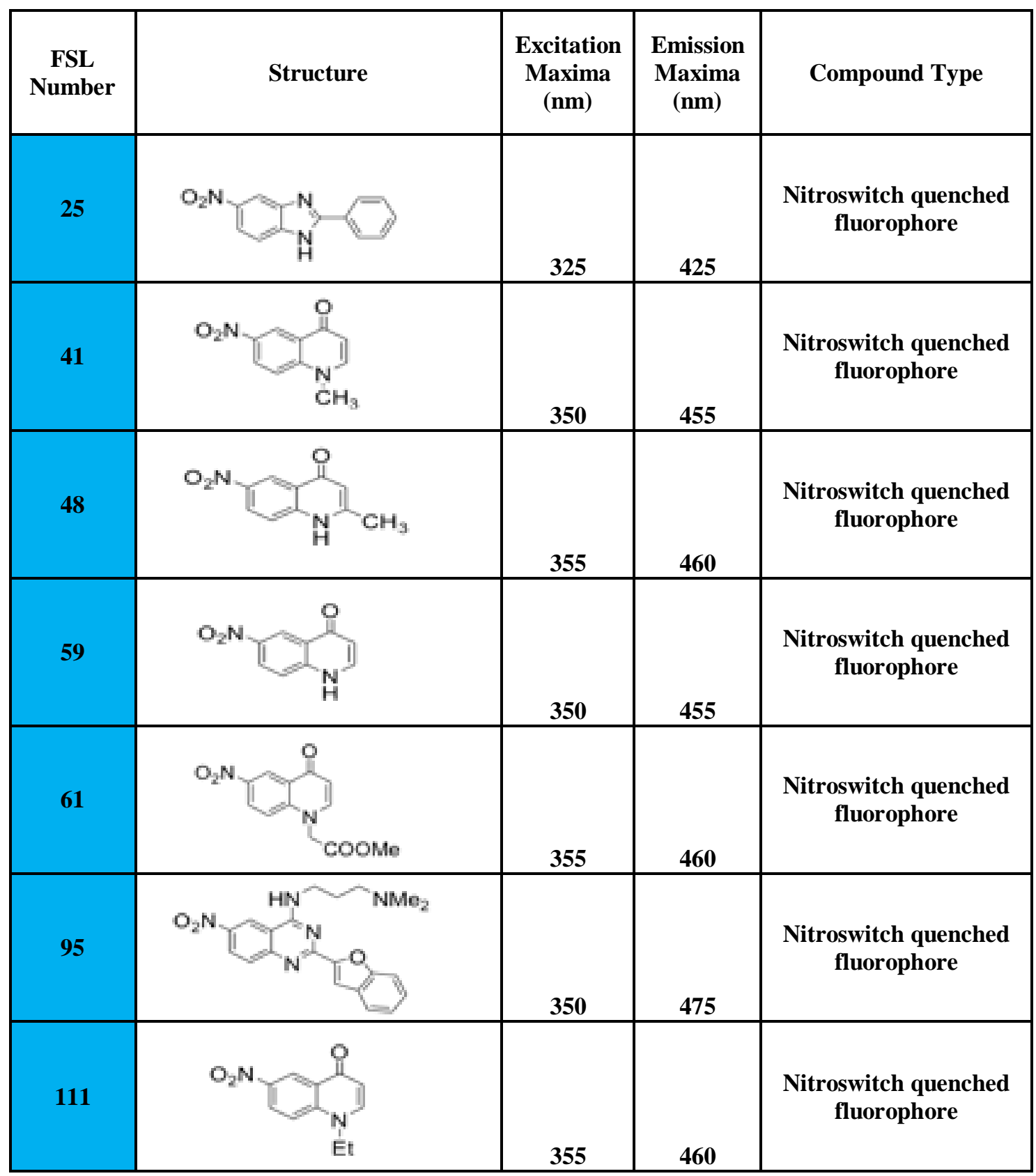




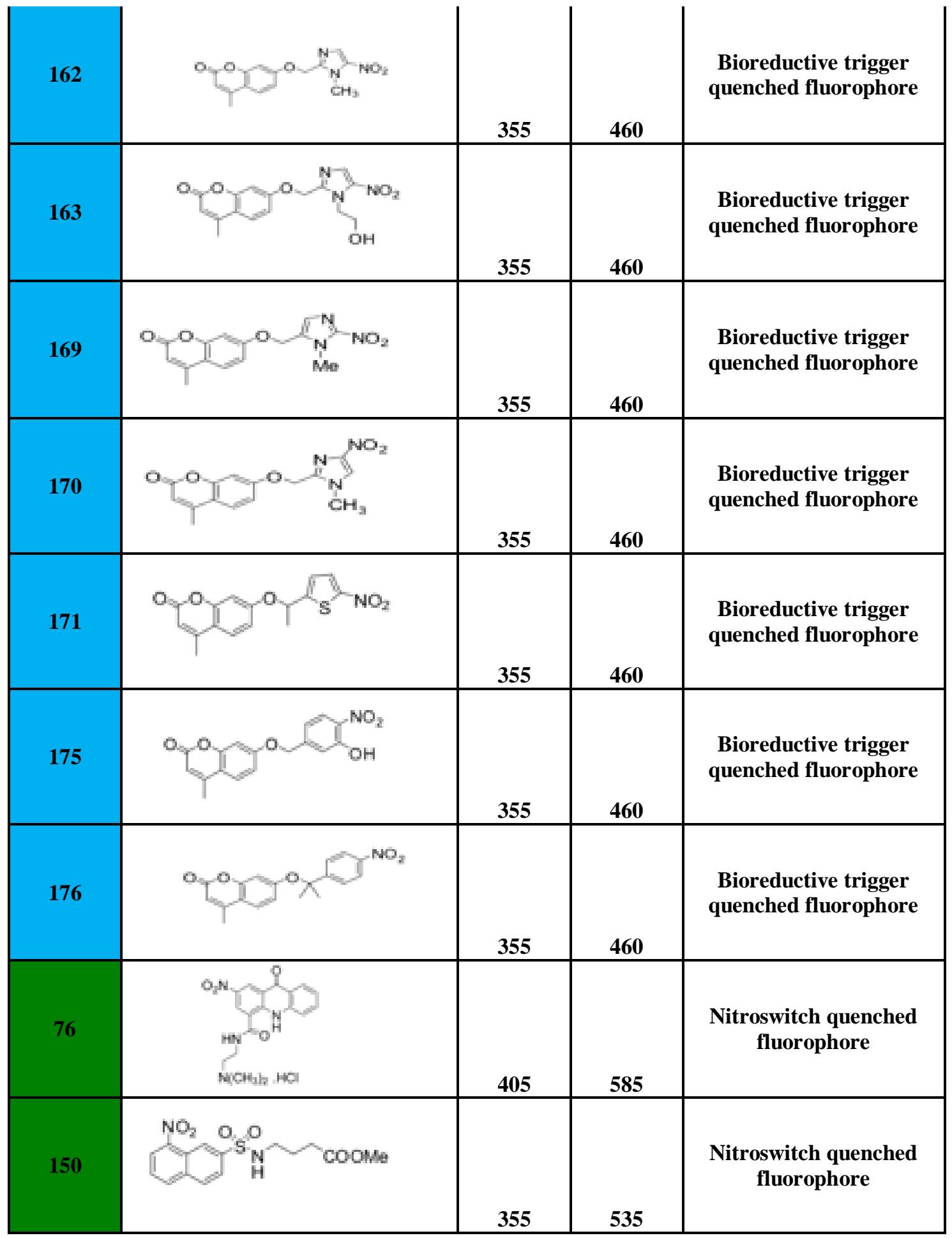




\begin{tabular}{|l|l|l|l|l|} 
& & & & $\begin{array}{c}\text { Boreductive trigger } \\
\text { quenched fluorophore }\end{array}$ \\
\hline 178 & & 645 & 660 & \\
\hline
\end{tabular}

\subsection{Enzymes}

Restriction Endonucleases and Phusion ${ }^{\mathrm{TM}}$ High Fidelity Polymerase were purchased from New England Biolabs (NEB; Ipswich, MA, USA). Bioline Red Taq Polymerase Mastermix was purchased from Bioline (London, UK). T4 DNA ligase was purchased from Fermentas (Burlington, Canada) . Advantage 2 Polymerase was purchased from Clontech (Mountain View, CA, US).

\subsection{Bacterial strains and plasmids}

Table 2.3 Bacterial Strains

\begin{tabular}{|c|c|c|}
\hline Strain & Characteristics & Source \\
\hline W3110 & E. coli $\mathrm{K} 12$ : F- $\lambda$ - IN(rrnD-rrnE) $1 \mathrm{rph} 1$ & $\begin{array}{l}\text { Ackerley Lab } \\
\text { Stock }\end{array}$ \\
\hline DH5a & $\begin{array}{l}\text { supEhh DlacU169 (980lacz DM5) hsd DM5) hsd } \\
\text { R17 }\end{array}$ & Invitrogen \\
\hline ADA510 & F- lac-6(del) $\lambda \phi($ sfiA::lacZ) & $\begin{array}{l}\text { (Shapiro \& } \\
\text { Baneyx, 2002) }\end{array}$ \\
\hline SOS-R2 & ADA510 $\Delta n f s A \Delta n f s B \Delta u v r B \Delta t o l C$ & $\begin{array}{l}\text { (Prosser et al, } \\
\text { 2013) }\end{array}$ \\
\hline W3110-6KO & W3110 $\Delta n f s A \Delta n f s B \Delta y i e F \Delta y c a K \Delta m d a B \Delta a z o R$ & (Horvat, 2012) \\
\hline 7NT & $\mathrm{W} 3110-7 \mathrm{KO} \quad \Delta$ nemA $\Delta$ tolC & Dr Janine Copp \\
\hline
\end{tabular}

Table 2.4 Plasmids

\begin{tabular}{lll}
\hline Plasmid & Characteristics & Source \\
\hline pUCX & ampR. E. coli expression vector. tac promoter, lac & (Prosser et al., \\
& operator, pET28a (+) RBS & 2010a) \\
pUC19 & ampR. Cloning plasmid, pMB1 origin of replication & Invitrogen \\
\hline
\end{tabular}




\subsection{Mammalian cell lines and plasmids}

Table 2.5 Mammalian cell lines

\begin{tabular}{|c|c|c|}
\hline Cell Line & Characteristics & Source \\
\hline HEK293 & $\begin{array}{l}\text { Human embryonic kidney, } \\
\text { adherent }\end{array}$ & $\begin{array}{l}\text { ATCC (American Type Culture } \\
\text { Collection, Manassas, VA, USA) }\end{array}$ \\
\hline HCT-116 & $\begin{array}{l}\text { Colorectal carcinoma, } \\
\text { adherent }\end{array}$ & $\begin{array}{l}\text { ATCC (American Type Culture } \\
\text { Collection, Manassas, VA, USA) }\end{array}$ \\
\hline HCT-116 GW NfsB_Vh & HCT-116 F279V5 $n f s B$ Vh & Dr Horvat \\
\hline HEK293 GW NfsA_Ec & HEK293 F279V5 nfsA Ec & This study \\
\hline HEK293 GW NfsB_Ec & HEK293 F279V5 $n f s B$ Ec & This study \\
\hline HEK293 GW NfsB_Vv & HEK293 F279V5 $n f s B$ Vv & This study \\
\hline HEK293 GW NfsA_Pp & HEK293 F279V5 nfsA Pp & This study \\
\hline
\end{tabular}

Table 2.6 Plasmids

\begin{tabular}{lll}
\hline Plasmid & Characteristics & Source \\
\hline F279V5 & Puromycin resistance & Dr Adam Patterson, \\
& & ACSRC \\
pDONR221 & $k a n R$ & Invitrogen \\
\hline
\end{tabular}

2.5 Oligonucleotide primers

Table 2.7 Oligonucleotide Primers

\begin{tabular}{|c|c|}
\hline Primer & Sequence $\left(5^{\prime} \rightarrow 3^{\prime}\right)$ \\
\hline \multicolumn{2}{|c|}{ NTR specific primer } \\
\hline NfsA_Ec_Fw & GGCATATGACGCCAACCATTGAAC \\
\hline NfsA_Ec_Rv & GGGTCGACTTAGCGCGTCGCCCAACCCTG \\
\hline NfsA_St_Fw & GGGGCATATGAGTCCGACCATTGAA \\
\hline NfsA_St_Rv & GGGGTCGACTTAGCGCGTCGCCCAGCC \\
\hline NfsA_Ck_Fw & GGGGCATATGACTCCAACCATTGATTT \\
\hline NfsA_Ck_Rv & GGGGGTCGACTTAGCGCGTCGCCCAA \\
\hline NfsA_Kp_Fw & GGGGCATATGACGCCGACCATTGAG \\
\hline NfsA_Kp_Rv & GGGGTCGACTCAGCGCGTTGCCCACC \\
\hline NfsA_Ecaro_Fw & GGGGCATATGATACCAACTATTGATTTGCTACA \\
\hline NfsA_Ecaro_Rv & GGGGCTCGAGCTAGCGTATCGCCCATCCTTGTTG \\
\hline NfsA_Es_Fw & CCCCCATATGACGCCAACGATTGAGCTGC \\
\hline NfsA_Es_Rv & GGGGCTCGAGTTAGCGTGTCGCCCAGCCCTG \\
\hline NfsA_Vf_Fw & CCCCATATGAACCCTGTTATCGATAC \\
\hline NfsA_Vf_Rv & CCCGTCGACTCACTTAATAGCTAGGC \\
\hline NfsA_Vv_Fw & GGGGCATATGAACGCTGTTATTGAC \\
\hline
\end{tabular}




\begin{tabular}{|c|c|}
\hline NfsA_Vv_Rv & GGGGTCGACTTACTTTGTCGCCAAGCC \\
\hline Frp_Vh_Fw & GGGCATATGAACAATACGATTGAAA \\
\hline Frp_Vh_Rv & GGGGTCGACTTAGCGTTTTGCTAGCC \\
\hline NfrA_Bs_Fw & GGGGCATATGAATAACACAATCGAAACC \\
\hline NfrA_Bs_Rv & GGGGGTCGACTTAGTTTTTATTAAAGCCCT \\
\hline NfsA_Li_Lw_Fw & GGGGCATATGAATCAGGCGATAGATGCCATTTT \\
\hline NfsA_Li_Lw_Rv & GGGGGTCGACTTATTTTTGATTTAAATGTTGC \\
\hline NfsA_Bc_Fw & GGGGCATATGAATACTATCATTGAAACGATTCTC \\
\hline NfsA_Bc_Rv & GGGGGTCGACTTACTTTTTATCAAACCCTTGGCGT \\
\hline NfsA_NP_Fw & GGCATATGCCTTTACAGATGGAA \\
\hline NfsA_NP_Rv & GGGTCGACTTACAGTAGCTTGAA \\
\hline NfsA_Bth_Fw & GGGGCATATGAATGAAATGATACATAAAATGGAG \\
\hline NfsA_Bth_Rv & GGGGGTCGACTCATTTCTTATTTAATCCTCTCTCAT \\
\hline NfsA_Lsak_Fw & GGGGCATATGTCTGATTTAATCGCACAAATGCAAC \\
\hline NfsA_Lsak_Rv & GGGGGTCGACTTATGCTAATGTAAACCCCTGTTTCTT \\
\hline NfsA_Pp_Fw & CCCCㄷTATGAGCCTTCAAGACGAAG \\
\hline NfsA_Pp_Rv & CTAGGTCGACTCAGCGCAGGCCGAAAC \\
\hline YcnD_Bs_Fw & CCCCCATATGAATGAAGTGATTAAATC \\
\hline YcnD_Bs_Rv & CCCCGTCGACTTATTTTTCAACTTTAAATC \\
\hline NfsA_Ms_Fw & GGGGCATATGACGGTCATCGCGCGCTACGCAGACGTCGAT \\
\hline NfsA_Ms_Rv & GGGGCTCGAGTCAGCGGATTCCCAGGCCCAACCGCTCG \\
\hline NfsB_Ec_Fw & GGGCATATGGATATCATTTCTG \\
\hline NfsB_Ec_Rv & GGGGAATTCTTACACTTCGGTTAAG \\
\hline NfsB_Ck_Fw & CCCCCATATGGATATCGTTTCTGTCGC \\
\hline NfsB_Ck_Rv & CCCGTCGACTCAGACTTCCGTCAGGGTG \\
\hline NfsB_St_Fw & GGGGCATATGGATATCGTTTCTGTC \\
\hline NfsB_St_Rv & GGGGGTCGACTTAGACTTCCGTCAGTG \\
\hline NfsB_Kp_Fw & GGGGCATATGGATATCGTATCGGTC \\
\hline NfsB_Kp_Rv & GGGGCTCGAGTTAAATCTCGGTAATGGT \\
\hline NfsB_Vv_Fw & GGGGCATATGACTATTGTTCAAGCT \\
\hline NfsB_Vv_Rv & GGGGTCGACTTAGATTTCGGTAAAAACAG \\
\hline NfsB_Pp_Fw & GGGGCATATGGATACCGTATCGCTG \\
\hline NfsB_Pp_Rv & GGGGCTCGAGTCAGAGGAAGGTGAACACTT \\
\hline NfsB_Es_Fw & CCCСCATATGAACCTTAATGAGATCATTCGC \\
\hline NfsB_Es_Rv & CCCGTCGACTCAGAGCTGCGTGATCAC \\
\hline FraseI_Vf_Fw & GGGGCATATGACGCATCCAATTATTCA \\
\hline FraseI_Vf_Rv & GGGGGTCGACCTAAAGAATGGTAATTAC \\
\hline NfsB_Vh_Fw & CССССАTATGTCTCATCAAATCATTACAGAC \\
\hline NfsB_Vh_Rv & CCCCTCGAGTTAAAGAGTAGTAATTACGTC \\
\hline YfkO_Bs_Fw & GGGGCATATGGCAGATCTAAAGACACA \\
\hline YfkO_Bs_Rv & CTAGGTCGACTTAAACCCACTTCACAACAT \\
\hline YdgI_Bs_Fw & GGGGCATATGATCAAAACAAACGATTTTATGG \\
\hline YdgI_Bs_Rv & GGGGGTCGACTTATTTCCATTCTGCAATTG \\
\hline YdgI_Pa_Fw & GGGGCATATGCATATCGAAGACGCC \\
\hline
\end{tabular}




\begin{tabular}{|c|c|}
\hline YdgI_Pa_Rv & GGGGGTCGACTCAGAAGCGGTCGCGAATGA \\
\hline AzoR_Ec_Fw & GGGGCATATGAGCAAGGTATTAGTTCTT \\
\hline AzoR_Ec_Rv & GGGGGTCGACTTATGCAGAAACAATGCTGT \\
\hline AzoR_St_Fw & CCCGCATATGAGCAAGGTATTAGTTCTT \\
\hline AzoR_St_Rv & CCCGTCGACTTAAGCGGCCACGAC \\
\hline AzoR_Vv_Fw & CCCGCATATGTCTCGTTTACTAGTATT \\
\hline AzoR_Vv_Rv & CCCGGTCGACTTAAGCTGTTAGCGCTGCAAG \\
\hline AzoR_Pp_Fw & GGGGCATATGTCCCGCGTACTGATCATC \\
\hline AzoR_Pp_Rv & GGGGCTCGAGTCAGGCCACCGCTGCCAGCTT \\
\hline NemA_Ec_Fw & CTAGGGATCCATGTCATCTGAAAAACTG \\
\hline NemA_Ec_Rv & CTAGGTCGACTTACAACGTCGGGTAATC \\
\hline NemA_St_Fw & CCCGGATCCATGTCATCAGCAAAACTG \\
\hline NemA_St_Rv & CCCGGTCGACTTACAGAGTAGGGTAGTC \\
\hline NemA_Kp_Fw & CCCCATATGTCGGAAGCAA \\
\hline NemA_Kp_Rv & CCCCTCGAGTTACAGAGTCGGGTAA \\
\hline NemA_Vv_Fw & CCCCATATGAGCAAACTGTTTGAA \\
\hline NemA_Vv_Rv & CCCGTCGACTCACACATGCAAAGCGGT \\
\hline YwrO_Bs_Fw & AAATTTGGATCCATGAAAATATTGGTTTTGGC \\
\hline YwrO_Bs_Rv & CCCGTCGACTTAAACAAAAGGCTGCTG \\
\hline YwrO_Li_Fw & CCCCCATATGAAAACATTAGTTATTAT \\
\hline YwrO_Li_Rv & CCCGTCGACCTAATTTAACGTTTTAATA \\
\hline YwrO_Vf_Fw & AAATTTGGATCCATGACAAGCCCGGCT \\
\hline YwrO_Vf_Rv & CCCGTCGACTTATTCCTTAATAAATTTATC \\
\hline YieF_Ec_Fw & GGGGCATATGTCTGAAAAATTGCAGGTG \\
\hline YieF_Ec_Rv & GGGGGTCGACTTAGATCTTAACTCGCTG \\
\hline YieF_Pa_Fw & GGGGCATATGAGCGACGACATCAAG \\
\hline YieF_Pa_Rv & GGGGGTCGACTCAACCGCGCAGGCGGCGCA \\
\hline MdaB_Ec_Fw & GGGCATATGAGCAACATCCTG \\
\hline MdaB_Ec_Rv & GGGGTCGACTTAACCAAAAATTTC \\
\hline MdaB_Ps_Fw & GGGGCATATGAAAAAAGTATTGTTCCTCAAC \\
\hline MdaB_Ps_Rv & CTAGCTCGAGTTACTGCGCACCAAACACTT \\
\hline WrbA_Ec_Fw & GGGCATATGGCTAAAGTTCTGGTG \\
\hline WrbA_Ec_Rv & GGGGTCGACTTAGCCGTTAAGTTT \\
\hline WrbA_Ps_Fw & GGGGCATATGAGCAAACCTTACATTCTGG \\
\hline WrbA_Ps_Rv & CTAGGTCGACTCAGCTGCGTGTTGTGTC \\
\hline YdjA_Ec_Fw & CTAGCATATGGATGCACTCGAACTATTG \\
\hline YdjA_Ec_Rv & CTAGGTCGACTCAGAAATAAGTTACAAACGG \\
\hline YdjA_Kp_Fw & CCCCCATATGGATGCTCTCGATCTACTGC \\
\hline YdjA_Kp_Rv & CCCCGTCGACTCAGAATCGCGTCACAAACG \\
\hline YdjA_Pp_Fw & GGGGCATATGCTTGTGAATGTACTG \\
\hline YdjA_Pp_Rv & GGGGGTCGACTCACGTCAGCGGGTACAACG \\
\hline YdjA_Pa_Fw & GGGGCATATGAACGTACTGATCGTC \\
\hline YdjA_Pa_Rv & GGGGGTCGACTCAGCGCGCCAGCGGCTGGA \\
\hline YcdI_Ec_Fw & CTAGCATATGAACGAAGCCGTTAGCC \\
\hline
\end{tabular}




YcdI_Ec_Rv
YcdI_Kp_Fw
YcdI_Kp_Rv
YcaK_Ec_Fw
YcaK_Ec_Rv
0853 Pa_Fw
0853_Pa_Rv
KefF_Ec_Fw
KefF_Ec_Rv
Miscellaneous primers
pMMB_Fw
pMMB_Rv
M13_Fw
M13_Rv

\section{Gateway Primers}

NfsA_Bth_Gateway_Fw

NfsA_Bth_Gateway_Rv

NfsA_Ecaro_Gateway_Fw

NfsA_Ecaro_Gateway_Rv

NfsA_Es_Gateway_Fw

NfsA_Es_Gateway_Rv

NfsA_Li_Gateway_Fw

NfsA_Li_Gateway_Rv

NfsB_Vh_Gateway_Fw

NfsB_Vh_Gateway_Rv

NfsB_Vv_Gateway_Fw

NfsB_Vv_Gateway_Rv
CTAGGTCGACTTACAACAGCCCGCAGG

GGGGCATATGAACGACGCGATAAACCA

CTAGCTCGAGTTATGCCAGGCAGGCT

GGGGCATATGCAGTCTGAACGTATT

GGGGGTCGACTTATAGTGCATCTACCATAT

GGGGCATATGGTTGAAACCGCCAAGACC

GGGGGTCGACCTAGACCTGGCTGCCGAGCT

GGGGCATATGATTCTTATAATTTATGCG

GCCTGAGTCGACGTATGGCTATCCATGATGGG

GGCTCGTATAATGTGTGG

GACCGCTTCTGCGTTCTGAT

GTAAAACGACGGCCAG

CAGGAAACAGCTATGAC

GGGGACAAGTTTGTACAAAAAAGCAGGCTT

CGAAGGAGATAGAACCATGGGCAATGAAAT

GATACATAAAAT

GGGGACCACTTTGTACAAGAAAGCTGGGTC

CTATTTCTTATTTAATCCTCTTT

GGGGACAAGTTTGTACAAAAAAGCAGGCTT

CGAAGGAGATAGAACCATGGGCATACCAAC

TATTGATTTGCT

GGGGACCACTTTGTACAAGAAAGCTGGGTC

CTAGCGTATCGCCCATCCTTGTT

GGGGACAAGTTTGTACAAAAAAGCAGGCTT

CGAAGGAGATAGAACCATGGGCACGCCAAC

GATTGAGCTGCT

GGGGACCACTTTGTACAAGAAAGCTGGGTC

CTAGCGTGTCGCCCAGCCCTGCT

GGGGACAAGTTTGTACAAAAAAGCAGGCTT

CGAAGGAGATAGAACCATGGGCAATCAGGC

GATAGATGCCAT

GGGGACCACTTTGTACAAGAAAGCTGGGTC CTATTTTTGATTTAAATGTTGCT

GGGGACAAGTTTGTACAAAAAAGCAGGCTT CGAAGGAGATAGAACCATGGGCTCTCATCA AATCATTACAGA

GGGGACCACTTTGTACAAGAAAGCTGGGTC CTAAAGAGTAGTAATTACGTCAT

GGGGACAAGTTTGTACAAAAAAGCAGGCTT

CGAAGGAGATAGAACCATGGGCACTATTGT

TCAAGCTGCCCA

GGGGACCACTTTGTACAAGAAAGCTGGGTC 


$\begin{array}{ll} & \text { CTAGATTTCGGTAAAAACAGTCT } \\ \text { O199_Gateway_Fw } & \text { GGGGACAAGTTTGTACAAAAAAGCAGGCTTCG } \\ & \text { AAGGAGATAGAACCATGGGCCCTTTACAGATG } \\ & \text { GAATTGGT } \\ \text { O199_Gateway_Rv } & \text { GGGGACCACTTTGTACAAGAAAGCTGGGTC } \\ & \text { CTACAGTAGCTTGAAGCCAAGAT } \\ \text { YdgI_Gateway_Fw } & \text { GGGGACAAGTTTGTACAAAAAAGCAGGCTT } \\ & \text { CGAAGGAGATAGAACCATGGGCATCAAAAC } \\ & \text { AAACGATTTATG } \\ \text { YdgI_Gateway_Rv } & \text { GGGGACCACTTTGTACAAGAAAGCTGGGTCC } \\ & \text { TATTTCCATTCTGCAATTTATC }\end{array}$

All primers used in this study were synthesised by Invitrogen (Carlsbad, CA, USA) or Integrated DNA Technologies (IDT; Coralville, IA, USA) and supplied lyophilized. For long-term storage, primers were resuspended in 1x TE, pH 8.0 (10 mM Tris-Cl $\mathrm{pH} 8.0,0.1 \mathrm{mM}$ EDTA) to a final concentration of $100 \mu \mathrm{M}$ and stored at $-20{ }^{\circ} \mathrm{C}$. For working stocks, aliquots were diluted in autoclaved, $0.22 \mu \mathrm{m}$ filter-sterilized distilled and deionised $\left(\mathrm{ddH}_{2} \mathrm{O}\right)$ water to a $10 \mu \mathrm{M}$ final concentration.

\subsection{Growth and maintenance of bacteria}

\subsubsection{Media}

All media listed below was made up to required volumes with $\mathrm{dd}_{2} \mathrm{O}$ then autoclaved at $121{ }^{\circ} \mathrm{C}$ for 30 minutes. Media was stored at room temperature unless supplemented with antibiotics or another heat sensitive chemical in which case it was stored at $4{ }^{\circ} \mathrm{C}$.

\subsubsection{Liquid media}

\section{Luria-Bertani (LB) Broth}

$1 \%$ Bacterial Peptone

$0.5 \%$ Yeast Extract

$1 \% \mathrm{NaCl}$

SOC

$0.5 \%$ Yeast Extract 
$2 \%$ Tryptone

$10 \mathrm{mM} \mathrm{NaCl}$

$2.5 \mathrm{mM} \mathrm{KCl}$

$10 \mathrm{mM} \mathrm{MgCl} l_{2}$

$10 \mathrm{mM} \mathrm{MgSO}_{4}$

$20 \mathrm{mM}$ Glucose

\subsubsection{Solid media}

Solid media was made by adding agar to LB at a final concentration of $1.5 \%$, then autoclaving. Supplements, when required, were added post-autoclaving once the media had cooled to $<50{ }^{\circ} \mathrm{C}$. Approximately $15 \mathrm{~mL}$ of medium was then poured into $90 \mathrm{~mm}$ x $15 \mathrm{~mm}$ Petri dishes and left to solidify. These were stored at $4{ }^{\circ} \mathrm{C}$ until use.

Table 2.8 Growth media supplements and antibiotics

\begin{tabular}{lll}
\hline & Final Concentration & Solvent \\
\hline Ampicillin & $100 \mathrm{mg} \mathrm{mL}^{-1}$ & $\mathrm{ddH}_{2} \mathrm{O}$ \\
Kanamycin & $100 \mathrm{mg} \mathrm{mL}^{-1}$ & $\mathrm{ddH}_{2} \mathrm{O}$ \\
IPTG & $100 \mathrm{mg} \mathrm{mL}^{-1}$ & $\mathrm{ddH}_{2} \mathrm{O}$ \\
Luria Broth & $20 \%$ & $\mathrm{ddH}_{2} \mathrm{O}$ \\
Glucose* & $20 \%$ & $\mathrm{ddH}_{2} \mathrm{O}$ \\
Glycerol & $80 \%$ & $\mathrm{ddH}_{2} \mathrm{O}$ \\
\hline
\end{tabular}

* Sterilised through a $0.22 \mu \mathrm{m}$ filter

\subsubsection{Growth of bacterial strains}

For standard growth of E. coli strains in liquid media, LB media (plus supplements if required) was inoculated and incubated at $37{ }^{\circ} \mathrm{C}$ with shaking at 200 revolutions per minute (rpm) unless otherwise stated. Solid media was incubated at $37{ }^{\circ} \mathrm{C}$ static and plates stored at $4{ }^{\circ} \mathrm{C}$ for up to a month. If long term storage of a strain was required liquid cultures of bacteria were mixed at a 1:1 ratio with $80 \%$ glycerol and stored at $-80{ }^{\circ} \mathrm{C}$.

\subsection{Growth and maintenance of mammalian cell lines}




\subsubsection{Media}

Dulbecco's Modified Eagle Medium (DMEM) was purchased from Invitrogen (Carlsbad, CA, USA) and supplemented with 2\% 4-(2-Hydroxyethyl)piperazine-1ethanesulfonic acid sodium salt (HEPES), 10\% Fetal Calf Serum (FCS) and 2\% Penicillin/Streptomycin. Puromycin was also added to a final concentration of $2 \mu \mathrm{M}$ in media when required for selection and maintenance of nitroreductase transfected cell lines. All media was filter sterilised through a $0.22 \mu \mathrm{M}$ filter into $50 \mathrm{~mL}$ aliquots and stored at $4{ }^{\circ} \mathrm{C}$, and warmed to $37^{\circ} \mathrm{C}$ before use.

\subsubsection{Antibiotics}

Table 2.9 Antibiotics used in the growth of mammalian cell lines

\begin{tabular}{lll}
\hline Antibiotic & Stock Concentration & Solvent \\
\hline Puromycin & $10 \mathrm{mM}$ & $\mathrm{ddH}_{2} \mathrm{O}$ \\
Penicillin/ Streptomycin & $10 \mathrm{mM}$ & $\mathrm{ddH}_{2} \mathrm{O}$ \\
\hline
\end{tabular}

\subsubsection{Growth of mammalian cells}

To initiate growth of a mammalian cell line, a cryotube containing frozen cells was thawed and added to a T25 flask containing $15 \mathrm{~mL}$ of pre-warmed media. The flask was incubated overnight at $37^{\circ} \mathrm{C} 5 \% \mathrm{CO}_{2}$. The media was then removed and replaced with fresh media. Cells were left to grow until they reached approximately $80 \%$ confluency, at which point they were harvested using the standard trypsin/EDTA protocol (see 2.7.3.1), diluted and seeded into a new flask.

Cell lines requiring long term storage were grown in a T75 flask until confluency was reached then harvested using the standard trypsin/EDTA protocol (2.7.3.1). The cell number was calculated and the cells were resuspended in Fetal Calf Serum containing $10 \%$ DMSO to give a final concentration of $5 \times 10^{6}$ cells per mL. For storage $1 \mathrm{~mL}$ aliquots of cells were placed into cryotubes and frozen by reducing the temperature by one degree per minute. When a temperature of $-80{ }^{\circ} \mathrm{C}$ was reached the cryotubes were placed in liquid nitrogen for long-term storage. 


\subsubsection{Harvesting cells}

When cells reached approximately $80 \%$ confluency, media was aspirated and cells washed in $1 \times$ PBS. Sufficient $0.25 \%$ Trypsin/EDTA was added to cover the cell surface of the well or flask and incubated at $37^{\circ} \mathrm{C}$ for five minutes. Cells were removed from the culture dish surface using 1x PBS and transferred to a $15 \mathrm{~mL}$ or 50 $\mathrm{mL}$ sterile centrifuge tube as appropriate. Cells were then centrifuged at $1250 \mathrm{rpm}$ for 5 minutes to pellet the cells. The supernatant was discarded and cells re-suspended in $1 \mathrm{~mL}$ pre-warmed culture media. If exact numbers of cells were required, $10 \mu \mathrm{L}$ of cell suspension was removed and put in a clean $1.5 \mathrm{~mL}$ microfuge tube and mixed with $10 \mu \mathrm{L}$ of Trypan blue. Cells were then counted using a hemocytometer (Improved Neubauer, Hausser, Horsham, PA) and seeded into fresh media at the desired density.

\subsection{Standard molecular biology protocols}

\subsubsection{Chemically competent cells}

E. coli from glycerol stocks were inoculated in $3 \mathrm{~mL} \mathrm{LB}+10 \mathrm{mM} \mathrm{MgCl}_{2}$ and incubated overnight at $37{ }^{\circ} \mathrm{C}, 200 \mathrm{rpm}$. Overnight cultures were diluted 50-fold into $50 \mathrm{~mL} \mathrm{LB}+10 \mathrm{mM} \mathrm{MgCl}$, and incubated at $37{ }^{\circ} \mathrm{C}, 200 \mathrm{rpm}$ until the $\mathrm{OD}_{600}$ was between 0.3 and 0.5 . When the $\mathrm{OD}_{600}$ was in this range, the cells were transferred to ice for 10 minutes. The cells were then centrifuged at $4{ }^{\circ} \mathrm{C}, 4000 \mathrm{rpm}$ for 15 minutes, then re-suspended in $40 \mathrm{~mL}$ TFBI (table 2.1) and left on ice for $2 \mathrm{~h}$. The cells were then centrifuged again at $4{ }^{\circ} \mathrm{C}, 4000 \mathrm{rpm}$ for 15 minutes, and re-suspended in $4 \mathrm{~mL}$ TFBII (table 2.1) . Cells were then transferred to sterile microcentrifuge tubes in 100 $\mu \mathrm{L}$ aliquots and stored at $-80{ }^{\circ} \mathrm{C}$.

Table 2.10 Recipes for chemically competent cell buffers

\begin{tabular}{ll}
\hline TFB I* & Final Concentration \\
\hline Potassium Acetate & $30 \mathrm{mM}$ \\
$\mathrm{MnCl}_{2}$ & $50 \mathrm{mM}$ \\
$\mathrm{CaCl}_{2}$ & $10 \mathrm{mM}$ \\
Glycerol & $15 \%$ \\
\hline TFB II* & Final Concentration \\
\hline
\end{tabular}




\begin{tabular}{ll}
\hline $\mathrm{MOPS} \mathrm{pH} .0$ & $10 \mathrm{mM}$ \\
$\mathrm{CaCl}_{2}$ & $75 \mathrm{mM}$ \\
$\mathrm{KCl}$ & $10 \mathrm{mM}$ \\
Glycerol & $15 \%$ \\
\hline
\end{tabular}

* Sterilised through a $0.22 \mu \mathrm{m}$ filter and stored at $4{ }^{\circ} \mathrm{C}$

\subsubsection{Transformation protocol}

Competent cells were defrosted on ice from $-80{ }^{\circ} \mathrm{C}$ storage, $50-100 \mu \mathrm{L}$ were used for each transformation reaction. Between 1-3 $\mu \mathrm{L}$ of DNA at a concentration of 20$200 \mathrm{ng} / \mu \mathrm{L}$ was added, the resulting mixture was kept on ice for 3 minutes. The cells were then heat shocked at $42{ }^{\circ} \mathrm{C}$ for $90 \mathrm{~s}$ and put immediately back on ice for 45-60 minutes. Cells were plated on LB + (selection compound $)+$ agar plates and incubated overnight in a static $37^{\circ} \mathrm{C}$ incubator.

\subsubsection{Electrocompetent cells}

A single colony was used to inoculate $3 \mathrm{~mL}$ of $\mathrm{LB}$ and grown overnight at $37^{\circ} \mathrm{C} 200$ $\mathrm{rpm}$. Subsequently $100 \mu \mathrm{L}$ of the overnight culture was used to inoculate $10 \mathrm{~mL}$ of fresh LB and the new culture grown at $37{ }^{\circ} \mathrm{C} 200 \mathrm{rpm}$ to an $\mathrm{OD}_{600}$ of 0.4- 0.6. The cells were then pelleted by centrifugation at $4000 \mathrm{rpm}$ for 10 minutes at $4{ }^{\circ} \mathrm{C}$ and the supernatant removed. The cells were then re-suspended in $500 \mu \mathrm{L}$ of ice-cold $10 \%$ glycerol and transferred to a $1.5 \mathrm{~mL}$ sterile microcentrifuge tube. Cells were then centrifuged at 13,000 rpm for 30 seconds, the supernatant removed and cells resuspended in $500 \mu \mathrm{L}$ of ice cold $10 \%$ glycerol. This was repeated $2 \mathrm{x}$ with a final resuspension of the cells in $30 \mu \mathrm{L}$ of ice cold $10 \%$ glycerol. Cells were then immediately used for electroporation or stored for $2-4$ weeks at $-80{ }^{\circ} \mathrm{C}$.

\subsubsection{Electroporation protocol}

Electrocompetent cells were transferred to a $0.2 \mathrm{~mm}$ electroporation cuvette and 5-8 $\mu \mathrm{L}$ of DNA was added. The cells were then electroporated $(2.5 \mathrm{kV}, 25 \mu \mathrm{F}, 100 \Omega)$ 
using a Gene Pulser XcellTM electroporation machine (BioRad, Hercules, CS, USA) and $900 \mu \mathrm{L}$ of SOC was added to the cuvette, mixed with the cells and the cell mixture transferred to a $1.5 \mathrm{~mL}$ sterile microcentrifuge tube. Cells were then allowed to recover for $90 \mathrm{~min}$ at $37{ }^{\circ} \mathrm{C}, 200 \mathrm{rpm}$. Half of the cell suspension was then plated on LB agar plates with the appropriate antibiotic and the plates incubated at $37{ }^{\circ} \mathrm{C}$ overnight. The remaining cell suspension was left at room temperature overnight and plated onto agar plates the next day.

\subsubsection{Colony screening}

Colonies that grew from transformation or electroporation were screened for the correct gene/plasmid insert by PCR using gene specific or plasmid specific primers, with a small (just visible) "smudge" of cells obtained by dipping a pipette tip into a colony used as template. Colonies shown to have the correct insert were grown overnight in LB plus antibiotic. For storage an aliquot of this overnight culture was mixed with glycerol (final concentration $40 \%$ ) and stored at $-80{ }^{\circ} \mathrm{C}$.

\subsubsection{DNA quantification}

DNA concentration and purity was measured for purified PCR and plasmid products using a NanoDrop ND-1000 spectrophotometer (ThermoScientific, WA, MA, USA).

\subsubsection{Polymerase chain reaction (PCR)}

For most PCR reactions Bioline Biomix $\operatorname{Red}^{\mathrm{TM}}$ (London, UK) Taq polymerase or Phusion ${ }^{\mathrm{TM}}$ high fidelity polymerase was used; the former for the generation of general PCR products, and the latter for products to be used in cloning.

\subsubsection{PCR components}

\section{Table 2.11 PCR components}




\begin{tabular}{ll}
\hline Component & Volume \\
\hline Bioline Biomix Red & \\
Biomix red & $10 \mu \mathrm{L}$ \\
Primer $1(100 \mu \mathrm{M})$ & $1 \mu \mathrm{L}$ \\
Primer $2(100 \mu \mathrm{M})$ & $1 \mu \mathrm{L}$ \\
Template DNA & $1 \mu \mathrm{L}$ \\
$\mathrm{ddH}_{2} \mathrm{O}$ & $7 \mu \mathrm{L}$ \\
& \\
Phusion & \\
$5 x$ Phusion HF Buffer & \\
dNTPs & $4 \mu \mathrm{L}$ \\
Primer $1(100 \mu \mathrm{M})$ & $0.4 \mu \mathrm{L}$ \\
Primer $2(100 \mu \mathrm{M})$ & $1 \mu \mathrm{L}$ \\
Phusion Polymerase & $1 \mu \mathrm{L}$ \\
Template DNA & $0.2 \mu \mathrm{L}$ \\
dd ${ }_{2} \mathrm{O}$ & $1 \mu \mathrm{L}$ \\
\hline
\end{tabular}

\subsubsection{PCR parameters}

PCR reactions were run using cycle parameters as presented in Table 2.12. Annealing temperature was generally $5{ }^{\circ} \mathrm{C}$ lower than the lowest $\mathrm{T}_{\mathrm{m}}$ of the pair of primers used in the reaction.

Table 2.12 PCR parameters

\begin{tabular}{l|l}
\hline Bioline Biomix Red & \\
\hline $95{ }^{\circ} \mathrm{C} 5 \mathrm{~min}$ & \\
$95{ }^{\circ} \mathrm{C} 30 \mathrm{~s}$ & 30 cycles \\
$52-60{ }^{\circ} \mathrm{C}{ }^{\circ} \mathrm{C} 30 \mathrm{~s}$ & \\
$72{ }^{\circ} \mathrm{C} 1 \mathrm{~min} / \mathrm{kb}$ & \\
Hold at $16{ }^{\circ} \mathrm{C}$ & \\
\hline Phusion $^{\mathrm{TM}}$ & \\
\hline $98{ }^{\circ} \mathrm{C} 1 \mathrm{~min}$
\end{tabular}


$98{ }^{\circ} \mathrm{C} 15 \mathrm{~s}$

$52-60{ }^{\circ} \mathrm{C}{ }^{\circ} \mathrm{C} 30 \mathrm{~s}$

30 cycles

$72{ }^{\circ} \mathrm{C} 30 \mathrm{~s} / \mathrm{kb}$

$72{ }^{\circ} \mathrm{C} 5 \mathrm{~min}$

Hold at $16{ }^{\circ} \mathrm{C}$

\subsubsection{Agarose gel electrophoresis}

To analyse the size of PCR products and other DNA samples, an aliquot of the sample was run on a $1 \%$ agarose gel (1\% agarose dissolved in 1xTAE buffer) containing ethidium bromide $\left(1 \mu \mathrm{g} \mathrm{mL}{ }^{-1}\right)$ and submerged in 1xTAE buffer. The gel was run at 120-140 V for 30-40 minutes and then the DNA bands viewed under ultra-violet (UV) light.

\subsubsection{Restriction digests}

Restriction digests were set up as described in Table 2.13 to a final volume of $50 \mu \mathrm{L}$. They were incubated at $37{ }^{\circ} \mathrm{C}$ from $3 \mathrm{~h}$ to overnight depending on the restriction enzymes being used and the amount of DNA present in the reaction. After the desired amount of time the restriction digests were heat inactivated at the appropriate temperature and time as recommended by the manufacturer. The restriction buffer that gives optimal restriction enzyme activity was chosen according to the NEB website (http://www.neb.com/nebecomm/tech_reference/restriction_enzymes/buffer_activity_ restriction enzymes.asp accessed 20.09.2013).

Table 2.13 Restriction digest parameters

\begin{tabular}{ll}
\hline Component & Volume \\
\hline Restriction buffer 10x & $5 \mu \mathrm{L}$ \\
Bovine Serum Albumin (BSA 2mg mL $\left.{ }^{-1}\right)$ & $2.5 \mu \mathrm{L}$ \\
Restriction enzyme 1 & $1-1.5 \mu \mathrm{L}$
\end{tabular}


Restriction enzyme 2

DNA

$\mathrm{ddH}_{2} \mathrm{O}$
$1-1.5 \mu \mathrm{L}$

As applicable

To make up to $50 \mu \mathrm{L}$ final volume

\subsubsection{Sequencing}

All sequencing was carried out by Macrogen Inc. (Seoul, Korea).

\subsubsection{Miniprep protocol}

In a $15 \mathrm{~mL}$ Falcon tube $3 \mathrm{~mL}$ of LB plus antibiotic was inoculated with a single colony and grown overnight at $37{ }^{\circ} \mathrm{C} 200 \mathrm{rpm}$. The following day plasmid DNA was extracted from the cells using the Geneaid high-speed plasmid mini kit (Taiwan, ROC) following manufacturer's instructions.

\subsubsection{Midiprep protocol}

In a $250 \mathrm{~mL}$ conical flask $50 \mathrm{~mL}$ of LB plus antibiotic was inoculated with a single colony and grown overnight at $37{ }^{\circ} \mathrm{C} 200 \mathrm{rpm}$. The following day plasmid DNA was extracted from the cells using the Geneaid high-speed plasmid midi kit (Taiwan, ROC) following manufacturer's instructions.

\subsubsection{SDS PAGE protocol}

To carry out SDS PAGE $12 \%$ polyacrylamide gels were cast as follows; $5 \mathrm{~mL}$ of $12 \%$ separating gel (Section 2.8.15.1) was added to the gel cast, covered by $100 \%$ isopropanol and left to set (at least 30 minutes). Once set the isopropanol was poured off and sufficient 4\% stacking gel (Section 2.8.15.1) was poured in to cover the wells of a multi-well comb (roughly about $2 \mathrm{~mL}$ ). This was left to set.

Protein samples for SDS PAGE were prepared by adding an appropriate volume of $3 \mathrm{x}$ SDS loading buffer (Section 2.8.15.1) and then boiling at $95{ }^{\circ} \mathrm{C}$ for five minutes. The samples were then loaded into the wells of the gel and the gel run on a Bio-Rad 
Protean II apparatus (Hercules, CA, USA) in 1x SDS Run Buffer at $150 \mathrm{~V}$ for 1 hour. Gels were then removed from the plates and stained by submerging the gel in Coomassie blue stain (Section 2.8.15.1) and rocking for 30 minutes. The gel was then washed in tap water to remove excess stain and then destained using destain solution (Section 2.8.15.1) to give the desired contrast between protein bands and the surrounding gel.

\subsubsection{Recipes for SDS PAGE gels and buffers}

\section{2\% Separating Gel (Per $10 \mathrm{~mL})$}

$3.75 \mathrm{~mL} 1 \mathrm{M}$ Tris-Cl ( $\mathrm{pH} 8.8)$

$0.05 \mathrm{~mL} 20 \% \mathrm{SDS}$

$2.92 \mathrm{~mL} 40 \%$ Acrylamide Solution

$1.6 \mathrm{~mL} 2 \%$ Bis-acrylamide Solution

$2.83 \mathrm{~mL} \mathrm{ddH_{2 } \mathrm { O }}$

$100 \mu \mathrm{L} 10 \%$ ammonium persulphate (APS)*

$6 \mu \mathrm{L}$ Tetramethylethylenediamine (TEMED)*

\section{4\% Stacking Gel (Per $3 \mathrm{~mL})$}

$375 \mu \mathrm{L} 1 \mathrm{M}$ Tris-Cl (pH 6.8)

$15 \mu \mathrm{L} 20 \%$ SDS

$300 \mu \mathrm{L} 40 \%$ Bis/Acrylamide Mix

$2.3 \mathrm{~mL} \mathrm{ddH}_{2} \mathrm{O}$

$30 \mu \mathrm{L} \mathrm{10 \%}$ APS*

$5 \mu \mathrm{L}$ TEMED*

\section{3 x SDS Loading Buffer}

150 mM Tris-Cl (pH 6.8)

$6 \%$ SDS

0.3\% Bromophenol Blue

$30 \%$ Glycerol 
$300 \mathrm{mM} \beta$-Mercaptoethanol

\section{0 x SDS Run Buffer (per Litre)}

144 g Glycine

$30.3 \mathrm{~g}$ Tris

$10 \mathrm{~g}$ SDS

\section{Coomassie Blue Stain (per Litre)}

$2.5 \mathrm{~g} / \mathrm{L}$ Coomassie Brilliant Blue

$450 \mathrm{~mL} \mathrm{100 \%} \mathrm{Ethanol}$

$100 \mathrm{~mL}$ 100\% Acetic Acid

$450 \mathrm{~mL} \mathrm{ddH}_{2} \mathrm{O}$

\section{Destain (per Litre)}

$400 \mathrm{~mL}$ 100\% Methanol

$100 \mathrm{~mL}$ 100\% Acetic Acid

$500 \mathrm{~mL} \mathrm{ddH} \mathrm{H}_{2} \mathrm{O}$

*Added immediately prior to pouring gel.

\subsection{Bacterial cell protocols}

\subsubsection{SOS assay}

Cultures of SOSR-2 pUCX:nitroreductase cultures were inoculated from glycerol stocks and grown overnight ( 16 hours) in 96 well plates containing $200 \mu \mathrm{L} \mathrm{LB}+$ amp $(100 \mu \mathrm{g}$ $\left.\mathrm{mL}^{-1}\right)+0.4 \%$ glucose. The internal wells of the plate were each inoculated with a single nitroreductase from the 58 nitroreductase library (Table 1.1), plus an empty plasmid control, and plates were incubated overnight at $30{ }^{\circ} \mathrm{C}, 200 \mathrm{rpm}$. The following day, $15 \mu \mathrm{L}$ of overnight culture was added to $200 \mu \mathrm{L}$ of assay media (LB + amp $100 \mu \mathrm{g}$ $\mathrm{mL}-1,0.05 \mathrm{mM}$ IPTG, $0.2 \%$ glucose) in a new 96 well plate and incubated at $30^{\circ} \mathrm{C}$, $200 \mathrm{rpm}$ for 3 hours. Day cultures were then split into $100 \mu \mathrm{L}$ into new 96 well plates in duplicate, containing either $100 \mu \mathrm{L}$ challenge media (assay media + prodrug, $2 \%$ 
DMSO) or $100 \mu \mathrm{L}$ control media (assay media $+2 \%$ DMSO). The plates were then incubated for a further $3-4$ hours at $30{ }^{\circ} \mathrm{C} 200 \mathrm{rpm}$. Plates were then removed from the incubator and a $20 \mu \mathrm{L}$ cell aliquot was added to $130 \mu \mathrm{L}$ of $\mathrm{ZOB}$ buffer in a fresh 96 well plate to allow measurement of $\beta$-galactosidase activity. This plate was then incubated at $37^{\circ} \mathrm{C}$ until sufficient yellow colouration was observed. At this point, the reaction was halted by addition of $50 \mu \mathrm{L}$ of $1 \mathrm{M} \mathrm{Na}_{2} \mathrm{CO}_{3}$. The unchallenged and challenged plates had their absorbance recorded at $600 \mathrm{~nm}$ while the plates that were used for B-galactosidase assay had their absorbance readings at $420 \mathrm{~nm}$ and $550 \mathrm{~nm}$ on the plate reader (Enspire 2300 Multiplate Reader, PerkinElmer, Waltham, MA, USA). From these readings Miller units were calculated by the Miller equation $[$ Miller units $=($ OD420 $-[1.75 \times$ OD550] $) /($ OD600 $\times t \times v)]$.

Table 2.14 Recipes for SOS assay buffers

\begin{tabular}{|c|c|}
\hline 1 M Sodium Phosphate Buffer pH 7* & Final Concentration \\
\hline $\mathrm{Na}_{2} \mathrm{HPO}_{4}$ & $0.577 \mathrm{M}$ \\
\hline $\mathrm{NaH}_{2} \mathrm{PO}_{4}$ & $0.423 \mathrm{M}$ \\
\hline Z Buffer* & Final Concentration \\
\hline $\mathrm{Na}_{2} \mathrm{HPO}_{4}$ & $0.074 \mathrm{M}$ \\
\hline $\mathrm{NaH}_{2} \mathrm{PO}_{4}$ & $0.126 \mathrm{M}$ \\
\hline $\mathrm{MgSO}_{4}$ & $2 \mathrm{mM}$ \\
\hline CTAB & $399 \mathrm{mg} . \mathrm{L}^{-1}$ \\
\hline Sodium Deoxycholate & $199.5 \mathrm{mg} . \mathrm{L}^{-1}$ \\
\hline B-Mercaptoethanol** & $0.174 \mathrm{M}$ \\
\hline T Base B $^{* * *}$ & Final Concentration \\
\hline $\mathrm{K}_{2} \mathrm{HPO}_{4}$ & $0.08 \mathrm{M}$ \\
\hline $\mathrm{KH}_{2} \mathrm{PO}_{4}$ & $0.044 \mathrm{M}$ \\
\hline$\left(\mathrm{NH}_{4}\right)_{2} \mathrm{SO}_{4}$ & $15.1 \mathrm{mM}$ \\
\hline Tri Sodium Citrate & $1 \mathrm{gL}^{-1}$ \\
\hline ONPG & $8 \mathrm{mg} \cdot \mathrm{mL}^{-1}$ \\
\hline
\end{tabular}

${ }^{*}$ Combined (650 mL $1 \mathrm{M}$ Sodium Phosphate Buffer $\mathrm{pH}$ 7, $\left.300 \mathrm{~mL} \mathrm{Z} \mathrm{Buffer}\right)$ and stored at $4{ }^{\circ} \mathrm{C}$

** B-mercaptoethanol added just prior to use

*** Stored at $-\mathbf{2 0}{ }^{\circ} \mathrm{C}$

NB. 1 M Sodium Phosphate Buffer pH 7, Z Buffer, and T Base combined make up the ZOB buffer used in the SOS assay. ZOB buffer sufficient for one 96 well plate $(13 \mathrm{~mL})$ was comprised of $9 \mathrm{~mL} 1 \mathrm{M}$ Sodium Phosphate Buffer pH 7, 3.9mL Z Buffer, and $100 \mu \mathrm{L}$ T Base).

\subsubsection{Growth inhibition assay}


Bacterial cultures were grown overnight from glycerol stocks as previously described in section 2.9.1. The following day, $15 \mu \mathrm{L}$ of overnight culture was added to $200 \mu \mathrm{L}$ of assay media (LB + amp $100 \mu \mathrm{g} \mathrm{mL}^{-1}, 0.05 \mathrm{mM}$ IPTG, $0.2 \%$ glucose) in new 96 well plates and incubated at $30{ }^{\circ} \mathrm{C}, 200 \mathrm{rpm}$ for $3.5 \mathrm{~h}$. Day cultures were then split into $100 \mu \mathrm{L}$ into new 96 well plates in duplicate, containing either $100 \mu \mathrm{L}$ challenge media (assay media + nil bystander (concentration stated in results), $2 \%$ DMSO) or $100 \mu \mathrm{L}$ control media (assay media $+2 \%$ DMSO), the plates then had their absorbance recorded at $600 \mathrm{~nm}$, and were then incubated for a further $4 \mathrm{~h}, 30^{\circ} \mathrm{C}, 200$ $\mathrm{rpm}$, then the absorbance was again recorded at $600 \mathrm{~nm}$ from the plate reader (Enspire 2300 Multiplate Reader, PerkinElmer, Waltham, MA, USA). From these readings the percentage decrease in cell growth was deduced by comparing T4-T0 $\mathrm{OD}_{600}$ data of NTRs with compounds to the unchallenged plates.

\subsubsection{IC $_{50}$ analysis}

Bacterial cultures were grown overnight from glycerol stocks as previously described in section 2.9.1. The following day, $50 \mu \mathrm{L}$ of overnight culture was added to $1 \mathrm{~mL}$ of assay media (LB + amp 100 $\mu \mathrm{g} \mathrm{mL}^{-1}, 0.05 \mathrm{mM}$ IPTG, $0.2 \%$ glucose) in $15 \mathrm{~mL}$ falcon tubes and incubated at $30^{\circ} \mathrm{C}, 200 \mathrm{rpm}$ for $2.5 \mathrm{~h}$. Day cultures were then splint into new 96 well plates in duplicate, containing $40 \mu \mathrm{L}$ of challenge media (assay media + nil bystander (concentration stated in results), ,2\% DMSO) in a gradient concentration across 7 wells. and an eighth unchallenged well. The plates then had their absorbance recorded at $600 \mathrm{~nm}$, and were incubated for a further $4 \mathrm{~h}, 30{ }^{\circ} \mathrm{C}, 200$ $\mathrm{rpm}$, then the absorbance was again recorded at $600 \mathrm{~nm}$ from the plate reader (Enspire 2300 Multiplate Reader, PerkinElmer, Waltham, MA, USA). From these readings the $\mathrm{IC}_{50}$ was obtained using the statistical analysis software Prism ${ }^{\odot}$.

\subsubsection{Fluorescence assay}

Bacterial cultures were grown overnight from glycerol stocks as previously described in section 2.9.1. The following day, $15 \mu \mathrm{L}$ of overnight culture was added to $200 \mu \mathrm{L}$ of assay media (LB + amp 100 $\mu \mathrm{g} \mathrm{mL}^{-1}, 0.05 \mathrm{mM}$ IPTG, $0.2 \%$ glucose) in new 96 well plates and incubated at $30{ }^{\circ} \mathrm{C}, 200 \mathrm{rpm}$ for $3.5 \mathrm{~h}$. Day cultures were then split into $100 \mu \mathrm{L}$ into new 96 well plates in duplicate, containing either $100 \mu \mathrm{L}$ challenge 
media (assay media + fluorophore (concentration stated in results), , $2 \%$ DMSO) or $100 \mu \mathrm{L}$ control media (assay media $+2 \%$ DMSO), and incubated for $3-4 \mathrm{~h} 30{ }^{\circ} \mathrm{C}, 200$ $\mathrm{rpm}$. The absorbance was then read at three different excitation/ emission settings dependent on the masked fluorophore present; red fluorescence (ex 645/ em 660), green fluorescence (ex 405/ em 585), blue fluorescence (ex 355/ em 460) on the plate reader (Enspire 2300 Multiplate Reader, PerkinElmer, Waltham, MA, USA). The fluorescence level of the empty plasmid control strain, pUCX, was deducted from each nitroreductase: fluorophore fluorescence level to account for background fluorescence.

\subsubsection{Combined growth inhibition and fluorescence assays}

Bacterial cultures were grown overnight from glycerol stocks as previously described in section 2.9.1. The following day, $15 \mu \mathrm{L}$ of overnight culture was added to $200 \mu \mathrm{L}$ of assay media (LB + amp 100 $\mathrm{g} \mathrm{mL}^{-1}, 0.05 \mathrm{mM}$ IPTG, $0.2 \%$ glucose) in new 96 well plates and incubated at $30^{\circ} \mathrm{C}, 200 \mathrm{rpm}$ for $3.5 \mathrm{~h}$. Day cultures were then split into $100 \mu \mathrm{L}$ into new 96 well plates in duplicate, containing either $100 \mu \mathrm{L}$ challenge media (assay media + fluorophore (concentration stated in results), + nil bystander antibiotic (concentration stated in results), $+2 \%$ DMSO) or $100 \mu \mathrm{L}$ control media (assay media $+2 \%$ DMSO). The plates then had their absorbance recorded at $600 \mathrm{~nm}$ and were incubated for $3-4 \mathrm{~h} 30{ }^{\circ} \mathrm{C}, 200 \mathrm{rpm}$. The absorbance at $600 \mathrm{~nm}$ was then read again to evaluate growth inhibition, and also at three different excitation/ emission settings; red fluorescence ex 645/ em 660), green fluorescence (ex 405/ em 585), blue fluorescence on the plate reader (Enspire 2300 Multiplate Reader, PerkinElmer, Waltham, MA, USA). The fluorescence level of the empty plasmid, pUCX, was deducted from each nitroreductase: fluorophore fluorescence level to account for background fluorescence.

\subsubsection{Bacterial confocal microscopy}

Cultures were set up and challenged with either masked fluorophore(s) - as for the fluorescence assay above (Section 2.9.5) - or a combination of masked fluorophore(s) and a nil bystander antibiotic (concentrations stated in results), - as for combined growth inhibition and fluorescence assay above (Section 2.9.5). A $5 \mu \mathrm{L}$ of culture 
was collected and mixed with $5 \mu \mathrm{L}$ of $4 \%$ paraformaldehyde. The mixture was then placed in the centre of a microscope slide and covered with a cover slip. Slides were observed and photographed under the confocal microscope (Olympus FV1000) on the same day as they were made.

\subsubsection{Validation of liquid media protocol with potential to be used for novel nitroreductase discovery}

Bacterial cultures from glycerols were grown overnight in a $15 \mathrm{~mL}$ falcon $3 \mathrm{~mL} \mathrm{LB}+$ antibiotic. The following day $50 \mu \mathrm{L}$ of overnight culture was added to $3 \mathrm{~mL} \mathrm{LB}+100$ $\mu \mathrm{g} \mathrm{mL}^{-1} \mathrm{amp}+2.5 \mu \mathrm{M}$ niclosamide. The $\mathrm{OD}_{600}$ was measured at $0 \mathrm{~h}, 2 \mathrm{~h}, 4 \mathrm{~h}, 8 \mathrm{~h}$.

\subsubsection{Validation of solid media protocol with potential to be used for novel nitroreductase discovery}

Bacterial cultures from glycerols were grown overnight in a $15 \mathrm{~mL}$ falcon $3 \mathrm{ml} \mathrm{LB}+$ antibiotic. The following day the cells were pelleted by centrifugation at $1250 \mathrm{rpm}$. The pellet was washed and resuspended in $1 \mathrm{~mL}$ of $1 \mathrm{x}$ PBS. The culture was diluted $1 \times 10^{6}$ fold in $1 \times$ PBS $100 \mu \mathrm{L}$ of the dilution plated on solid media plates containing

of $100 \mu \mathrm{g} \mathrm{mL}^{-1} \mathrm{amp}, 0.05 \mathrm{mM}$ IPTG, $10 \mu \mathrm{M}$ metronidazole, and $2.5 \mu \mathrm{M}$ niclosamide After a $48 \mathrm{~h}$ growth period, colonies were picked and PCR screened to determine using Bioline Biomix Red ${ }^{\mathrm{TM}}$ as per components and parameters outlined in 2.8.8 and 2.8.9, as well as undergoing fluorescence screening as per 2.9.4 with masked fluorophore FSL41 (concentration stated in results),.

\subsection{Mammalian cell protocols}

\subsubsection{Construction of mammalian nitroreductase stable expression cell lines}


For experimental analysis of bacterial NTR activity in mammalian cells, mammalian cell lines stably expressing bacterial nitroreductases were generated in HEK293 cell lines (Table 2.5).

\subsubsection{Creating Gateway ${ }^{\circledR}$ constructs}

The Gateway primers as listed in Table 2.6 were used to amplify the NTR gene using the standard PCR method as described in Section 2.8.9. PCR reactions were then analysed by gel electrophoresis to check for a product of the correct size for the target NTR. The PCR reactions were then DNA purified using the Zymo DNA Clean and Concentrate $^{\mathrm{TM}}$ Kit (Zymo Research, Irvine, CA, USA).

\subsubsection{BP recombination}

The Gateway entry clone was then created through a BP recombination step using the pDONR221 (purchased from Invitrogen, Carlsbad, CA). The BP clonase II kit was also purchased from Invitrogen (Carlsbad, CA). The components of Table 2.12 were added to a $1.5 \mathrm{~mL}$ microfuge tube and then incubated at $25^{\circ} \mathrm{C}$ for at least twelve hours. After incubation $1 \mu \mathrm{L}$ of Proteinase $\mathrm{K}$ was added to each reaction tube and incubated at $37^{\circ} \mathrm{C}$ for 10 minutes. Each BP reaction was then transformed into DH5 $\alpha$ competent cells. Transformations were then plated on LB + kanamycin plates and incubated overnight at $37^{\circ} \mathrm{C}$.

Table 2.15 BP recombination reaction components

\begin{tabular}{lccc}
\hline Component & Sample & Positive Control & Negative Control \\
\hline PCR product (20- 50 fmol) & $1-7 \mu \mathrm{L}$ & - & - \\
pDONR221 vector $(150 \mathrm{ng} / \mu \mathrm{l})$ & $1 \mu \mathrm{L}$ & $1 \mu \mathrm{L}$ & $1 \mu \mathrm{L}$ \\
pEXP7-tet positive control $(50 \mathrm{ng} / \mu \mathrm{l})$ & - & $2 \mu \mathrm{L}$ & - \\
TE Buffer, pH 8.0 & Up to $8 \mu \mathrm{L}$ & $5 \mu \mathrm{L}$ & $7 \mu \mathrm{L}$ \\
\hline
\end{tabular}

\subsubsection{Analysing entry clones}

Five colonies were picked from the plates from the BP recombination step and cultured overnight in $3 \mathrm{~mL}$ LB + kanamycin. Plasmid DNA was then isolated from 
the overnight cultures using the miniprep protocol described in section (2.8.13). Restriction enzyme NheI was used to cut the plasmid to confirm the presence and correct orientation of the insert. Entry clones that showed the correct restriction pattern were then sequenced to confirm the correct insert was present.

\subsubsection{LR recombination}

The next step was to carry out the LR recombination step. The LR clonase II enzyme mix, proteinase K solution and pENTR-gus were all purchased from Invitrogen (Carlsbad, CA). The destination vector used in these experiments was F279/V5 which was created by Dr Adam Patterson at the ACSRC (Prosser et al., 2013). The components of the reaction as listed in Table 2.13 were added to $1.5 \mathrm{~mL}$ microfuge tubes and reactions then incubated at $25{ }^{\circ} \mathrm{C}$ for 12 hours.

After incubation $1 \mu \mathrm{L}$ of Proteinase $\mathrm{K}$ solution was added to each reaction and incubated at $37{ }^{\circ} \mathrm{C}$ for 10 minutes. Samples were either stored at $-20^{\circ} \mathrm{C}$ or $4 \mu \mathrm{L}$ of each LR recombination was transformed into DH5 $\alpha$ competent cells. Expression clones were selected for by plating on LB + ampicillin plates.

Table 2.16 LR recombination reaction components

\begin{tabular}{lcc}
\hline Component & Sample & Positive Control \\
\hline Entry clone $(50-150 \mathrm{ng} /$ reaction $)$ & $1-7 \mu \mathrm{L}$ & - \\
Destination Vector $(150 \mathrm{ng} / \mu \mathrm{L})$ & $1 \mu \mathrm{L}$ & 1 \\
pENTR-gus $(50 \mathrm{ng} / \mu \mathrm{L})$ & - & 5 \\
TE Buffer, $\mathrm{pH} 8.0$ & Up to $8 \mu \mathrm{L}$ & 5 \\
\hline
\end{tabular}

\subsubsection{Confirming the identity of F279/ V5 destination clones}

This was done through a restriction digest using the restriction enzyme HindIII and analyzing the result by gel electrophoresis. Confirmed expression clones were sequenced and then a midiprep of the construct carried out using the Geneaid highspeed plasmid midi kit (Taiwan, ROC). These constructs were then used to transfect cell lines of interest.

\subsubsection{Stable Transfection Cell Lines}




\subsubsection{Lipofectamine ${ }^{\mathrm{TM}} 2000$ reagent protocol}

250,000 HEK WT cells were seeded into a tissue culture treated 6 well plate containing $2 \mathrm{~mL}$ DMEM (life technologies) media with 5\% Fetal Calf Serum (FCS), $2 \%$ Penicillin/ Streptomycin, and 2\% HEPES. Plates were then incubated at $37{ }^{\circ} \mathrm{C} 5 \%$ $\mathrm{CO}_{2}$ for 24 hours.

For each transfection $4 \mu \mathrm{g}$ of DNA from the F279/ V5 constructs was diluted in 500 $\mu 1$ of serum free media and mixed thoroughly in a sterile microcentrifuge tube. In a separate sterile microcentrifuge tube, $8 \mu \mathrm{L}$ of the transfection reagent was added to $492 \mu \mathrm{L}$ of serum free media. These separate sterile microcentrifuge tubes were incubated at room temperature for 5 minutes and then combined and incubated at room temperature for a further 20 minutes.

After incubation the transfection mixture was added to the well containing the cells and mixed in gently. Cells were put back in the $37{ }^{\circ} \mathrm{C} 5 \% \mathrm{CO}_{2}$ incubator. Cell populations were then sub-cultured at least 3 times, with increasing concentrations of puromycin, to a final concentration of $2 \mu \mathrm{M}$.

\subsubsection{Making stocks of stable cell lines}

Cells from a 6 well plate that had almost grown to confluence after transfection were harvested and seeded into T75 cell culture flasks. Cells were left to grow at $37{ }^{\circ} \mathrm{C} 5 \%$ $\mathrm{CO}_{2}$ until $90 \%$ confluence was reached, and then harvested following the Trypsin/EDTA method. The cells were centrifuged at $1250 \mathrm{rpm}$ for 5 minutes to pellet. The supernatant was discarded and the pellet was then resuspended in PBS. Cells counted, and 5 million were added to $1 \mathrm{~mL}$ FCS and 10\% DMSO in cryotubes. The $1 \mathrm{~mL}$ aliquots were then frozen down at a rate of $-1{ }^{\circ} \mathrm{C}$ per minute. Once samples had reached $-80{ }^{\circ} \mathrm{C}$ they were transferred into liquid nitrogen for long-term storage.

\subsubsection{MTS assay}

The viability of mammalian NTR expression cell lines post incubation with nil 
bystander antibiotics was assessed using a standard MTS assay (ref a MTS assay paper). Cells were seeded (10,000 per well in $100 \mu \mathrm{L}$ of media) into a tissue culture treated 96 well plate. For each strain sufficient wells were seeded so that cell viability could be monitored in triplicate at $0,5,10,30,40$ and $80 \mu \mathrm{M}$ of nil bystander (unless otherwise stated) and following both 24 and 48 hours exposure to antibiotic.

Plates were incubated at $37^{\circ} \mathrm{C} 5 \% \mathrm{CO}_{2}$ overnight. Media was then aspirated from each well and replaced with $100 \mu \mathrm{L}$ of phenol free media supplemented withan appropriate concentration of nil bystander antibiotic.. Plates were returned to the incubator. After 24 hours one experimental plate set was removed from the incubator. To each well30 $\mu 1$ of MTS reagent (CellTitre 96 Aqueous One Solution Cell Proliferation Assay - Promega, Madison, WI, USA) was added. Plates were returned to the incubator for an hour. Plates were read on a plate reader at $590 \mathrm{~nm}$ (Enspire 2300 Multiplate Reader, PerkinElmer, Waltham, MA, USA). At 48 hours the remaining plates were removed and steps from the 24 hour time period repeated.

\subsubsection{Confocal microscopy in mammalian cells}

$5 \times 10^{6}$ cells were seeded into $35 \mathrm{~mm}$ Fluorodish Cell Culture Dishes (World Precision Instruments Inc., Sarasota, FL) in $1 \mathrm{~mL}$ phenol free media and incubated at $37{ }^{\circ} \mathrm{C} 5 \%$ $\mathrm{CO}_{2}$ overnight. Masked fluorophore was added to a final concentration of $50 \mu \mathrm{M}$ and the cells incubated for a further hour. Fluorescent cells were then visualized in the culture dishes using an Olympus FV1000 confocal microscope.

\subsubsection{Microscopy of challenged mammalian cells}

$2.5 \times 10^{4}$ cells were seeded into T25 cell culture flasks in $5 \mathrm{~mL}$ DMEM media and incubated at $37{ }^{\circ} \mathrm{C} 5 \% \mathrm{CO}_{2}$ overnight. Nil bystander antibiotic was added to a final concentration of $5 \mu \mathrm{M}$ and the cells incubated for a 48 hour period. Cells were then visualized on an Olympus IX51 Microscope.

\subsubsection{Confocal microscopy of challenged mammalian cells treated with masked}

\section{fluorophores}


$5 \times 10^{6}$ cells were seeded into $35 \mathrm{~mm}$ Fluorodish Cell Culture Dishes (World Precision Instruments Inc., Sarasota, FL) in $2 \mathrm{~mL}$ phenol free media and incubated at $37{ }^{\circ} \mathrm{C} 5 \%$ $\mathrm{CO}^{2}$ overnight. Nil bystander antibiotic was added to a final concentration of $150 \mu \mathrm{M}$ and the cells incubated for a further two hours. After one hour masked fluorophore was added to a final concentration of $25 \mu \mathrm{M}$ and the cells incubated for a further hour. Cells were then visualized in the culture dishes using an Olympus FV1000 confocal microscope. 


\section{Nil Bystander Antibiotics}

\subsection{Introduction}

This chapter outlines the results from screening of the 58 nitroreductase core library with the 16 chosen nil bystander antibiotics. The 58 nitroreductase core library was developed in the Ackerley lab and is a very valuable resource, as it encompasses a large variety of nitroreductases. The library is weighted heavily toward the two major characterised nitroreductase families; NfsA and NfsB. The 16 nil bystander antibiotics were chosen based on availability in the lab, as well as either known nilbystander prodrug activity or structural features consistent with such activity. All of the experiments undertaken in this thesis were based on the results observed in the growth inhibition screening assays (protocol 2.9.2).

The initial motivation for this work was to investigate the ability of nitroreductases to reduce a range of nitro-quenched nil-bystander antibiotics, to provide a potential safety mechanism for eliminating biological vectors from GDEPT patients posttherapy, or if PET imaging were to indicate undesirable vector localisation. This is a very desirable feature, as pathogenic microorganisms are the most commonly used vectors in solid tumour targeting. Due to their pathogenic origins the FDA requires that there be a way to eliminate these vectors from the patient. While bacteria such as Clostridium species are already sensitive to antibiotics such as metronidazole (and indeed, in the clinic are typically cleared with this antibiotic), heightened sensitivity is a very desirable feature. Moreover, whereas viruses are not generally sensitive to antibiotics, recent work by our collaborator Dr Adam Patterson has suggested that nitroreductase-mediated activation of potent nil-bystander prodrug antibiotics may provide a mechanism to clear viral vectors (Figure 1.6).

Contemporary use of nitroreductases has also explored the possibility of their use in developmental biology. Curado et al. (2007) engineered a system whereby a promoter drives the expression of a nitroreductase; in this case NfsB_Ec. When the nil bystander antibiotic metronidazole is added, it is converted to its cytotoxic form, inducing DNA damage in the cells in which the nitroreductase is present, resulting in cell death. The promoter is also fused (as a bicistronic construct) to a reporter gene 
such as GFP, which enables visualisation of the cells expressing the nitroreductase prior to the addition of metronidazole and the confirmation of nitroreductase activation after addition as the fluorescence ceases, indicating the cells with GFP and nitroreductase are no longer intact and expressing.

Previous experiments undertaken in the Ackerley lab however have suggested that NfsB_Ec has relatively low activity with metronidazole compared to other nitroreductases in our collection. As mentioned in section 1.9.?, NfsB_Ec, is insufficiently active with metronidazole to give clean targeted cell ablation without high background toxicity, thus, identification of superior nitroreductases for activation of metronidazole and/or superior nitroreductase/ nil bystander antibiotic combinations would be highly advantageous in this context. Achieving one or both outcomes was the initial goal of the research described in this thesis.

A more ambitious research goal was to expand on a uni-dimensional GFP: nitroreductase ablation system to identify pairs of nil bystander antibiotics/ nitroreductase $\mathrm{s}$ with opposing activation profiles. The idea behind this was the possibility of using these in a multiplexed scenario, to specifically target two distinct cell populations and observe the effects of ablation of each; either simultaneously or independently (as per Figure 1.11).

Thus, the research described in the first part of this chapter begins with initial screening in E. coli of the 58 nitroreductase core library against the 16 nil bystander antibiotics and the creation of a heat-map based on activation profiles observed in growth inhibition screens - allowing rapid identification of nitroreductases with high activation of nil bystander antibiotics, and also pairs of nitroreductases that exhibit opposing activation profiles with different nil-bystander antibiotics. This initial screen was followed up by a more detailed and more quantitative $\mathrm{IC}_{50}$ analysis of nitroreductase / nil bystander combinations that appeared to have potential for use in the dual ablation system.

The second part of this chapter explored the translation of results observed in bacterial cells into human cell lines. The ability of the nitroreductases to be active in 
eukaryotic cells is essential for their continued use in developmental and regenerative biology, and in GDEPT. Previous studies from the Ackerley lab have indicated that not all bacterial nitroreductases are tolerated in transgenic human cells, with approximately $50 \%$ of candidates unable to form stably transfected cell lines (Prosser et al., 2013). Thus, it was essential to test that results observed in bacterial assays were predictive of the phenotypes that would be observed in nitroreductase transfected eukaryotic cell lines.

During the initial screening in bacterial cells one of the candidate nil bystander antibiotics that was examined, niclosamide, appeared to have the opposite activation profile with the nitroreductase library than had been expected, i.e. was toxic to wild type $E$. coli but not to bacteria expressing active nitroreductases. The final section of this chapter explores this anomaly, and the potential of niclosamide to be exploited as a novel nitroreductase screening method.

\subsubsection{Nil bystander antibiotics}

The nil bystander antibiotic candidates that were tested in this study are summarised in table 2.1. As confirmation that a particular molecule does indeed exhibit a negligible bystander effect is a complex and time-consuming task (requiring 3D mixed cell cultures, e.g. Wilson et al, 2002), for this study candidate molecules were chosen based on historical significance, availability in the laboratory, and/or structural similarity to molecules that were already in use in the lab. Below, I will briefly discuss the grounds for inclusion of the nil bystander antibiotics.

These compounds should be non-toxic in their original state. However, the reduction of their nitro group leads to the production of short-lived cytotoxic intermediates. The toxicity of the intermediates is due to their interaction with deoxyribonucleic acid and possibly with other macromolecules (Muller, 1983). Metronidazole is one of the most well-studied of the nil bystander antibiotics used in this research. It has been implemented medically for over 45 years (Löfmark et al, 2010) and is capable of wiping out anaerobic bacterial populations. Metronidazole's mechanism of action is not fully elucidated; it is readily taken up by obligate anaerobic organisms and is subsequently reduced by low-redox potential electron-transport proteins to an active, 
cytotoxic intermediate product. The reduced product of metronidazole causes DNA strand breaks, thereby inhibiting DNA synthesis and bacterial cell growth (http://www.drugbank.ca/drugs/DB00916, accessed 20.08.2013).

Tinidazole is also a well-studied antibiotic. It has antiprotozoal properties, and its nitro group is reduced upon activation, generating a series of free nitro radicals. Toxicity is achieved via depletion of sulfhydryl groups and DNA strand breaks, with multiple hits having an additive effect, ultimately leading to cell death (http://www.drugbank.ca/drugs/DB00911 accessed 20.08.2013). It was originally used in the treatment of several protozoal infections (Sawyer et al., 1976) but as with all of these molecules the potential to damage DNA upon activation gives it generic cell ablation capabilities.

Another advantage of metronidazole and tinidazole is that they have been shown to be capable of targeted ablation of individual bacteria or virus infected cells without localised tissue damage, and that they are able to be activated by two common nitroreductases NfsA_Ec and NfsB_Ec (Prosser et al., 2010b). Researchers at the Auckland Cancer Society Research Centre (ACSRC) have also been able to show that metronidazole has the ability to suppress the replication of the ONYX-411 adenovirus in cultured human cell lines when it is labelled with $n f s B \_E c$, but not the wildtype ONYX-411 adenovirus (Figure. 1.6). This revelation has made these two nil bystander antibiotics the most focused on throughout this thesis.

Misonidazole and F-misonidazole are both nitroimidazole radiosensitisers. They induce the formation of free radicals and deplete thiols, consequently sensitising hypoxic cells to the cytotoxic effects of ionizing radiation induced by single strand breaks in DNA resulting in the inhibition of DNA synthesis (http://www.cancer.gov/drugdictionary?cdrid=39504 accessed 20.08.2013, http://www.cancer.gov/drugdictionary?cdrid=458064 accessed 20.08.2013). Fmisonidazole has the added potential to be labelled with the radionuclide ${ }^{18} \mathrm{~F}$ to enable PET imaging of hypoxia (http://imaging.cancer.gov/programsandresources/fmisodocumentation accessed 02.09.2013). 
EF5 is a fluorinated derivative of the 2-nitroimidazole etanidazol. Reduction of EF5 is carried out by a diverse group of enzymes in the cytoplasm, microsomes and mitochondria. Tissue hypoxia detection via EF5 has been seen in several cancers, including squamous cell carcinoma of the cervix and the head and neck, and in sarcoma (Komar et al., 2008).

Dimetridazole, ornidazole, and ronidazole have antiprotozoal and antibacterial properties, and are most commonly used in the agricultural sector to prevent bacterial infections, cure protozoan infections, and for the treatment of swine dysentery respectively (Moreno \& Docampo, 1985). Ornidazole has also been explored as a treatment for Crohn's disease and is commonly used as an antimicrobial agent in patients undergoing gastrointestinal surgeries (Steib et al., 1993).

Furazolidone is a broad-spectrum nitrofuran with antiprotozoal and antibacterial properties. It has been used in both human and veterinary medicine. It works by binding bacterial DNA which leads to the gradual inhibition of monoamine oxidase (http://www.drugbank.ca/drugs/DB00614 accessed 20.08.2013).

Niclosamide is a taeniacide, a substance used to eradicate tapeworms, which is used for the treatment of most tapeworm infections (http://www.drugbank.ca/drugs/DB06803 accessed 20.08.2013). More recently it has been explored for potential use in cancer treatment (Sack et al., 2011, Osada et al., 2011).

Nifurtimox is a nitrofuran derivative with antiprotozoal characteristics. It is reduced by cytosol enzymes or flavin-containing microsomal enzymes to a highly reactive nitro anion free radical. These derivatives alkylate macromolecules such as nucleic acids and proteins, resulting in the disruption of their structure and function. (http://www.cancer.gov/clinicaltrials/search/view?cdrid=586650\&version=HealthProf essional accessed 20.08.2013) Along with $N$-benznidazole, nifurtimox is commonly used for the treatment of Chagas disease (Castro and Diaz de Toranzo 1988). 
Nitrofurantoin is a broad spectrum antibacterial that is active against some grampositive and gram-negative organisms. It is commonly used for treating urinary tract infections, but is of particular interest due to its high stability to the development of bacterial resistance, a property thought to be due to its multiplicity of mechanisms of action. (http://www.drugbank.ca/drugs/DB00698 accessed 20.08.2013).

Nitrofurazone is a topical anti-infective agent effective against gram-negative and gram-positive bacteria. It is most commonly used for superficial wounds, burns, ulcers, and skin infections, although it has also been administered orally in the treatment of trypanosomiasis (http://www.drugbank.ca/drugs/DB00336 accessed $\underline{20.08 .2013}$ ).

2-Methyl-5-Nitroimidazole and 4-Nitroimidazole are generic imidazole derivatives, which are routinely used in the treatment of many systemic fungal infections (Gunay et al., 1999, Benkli et al., 2003)

\subsubsection{Nitroreductase library}

The core nitroreductase library in the Ackerley lab was constructed by various past and present lab members in the E.coli SOS-R2 strain (table 2.3). Preliminary nitroreductases were chosen from the NCBI database through BLAST homology searches using each of the E. coli enzymes as well as human NQO1 and YwrO as query sequences. The library was categorised into separate families based around these key members. Nitroreductases were chosen from a range of bacterial species based on availability in the Ackerley laboratory; this included both Gram-positive and Gram-negative strains, with the only other criteria for selection being sharing at least $25 \%$ amino acid identity with the key family member. Nitroreductase enzymes that were identified were subsequently cloned into the plasmid pUCX (Figure 3.1). 


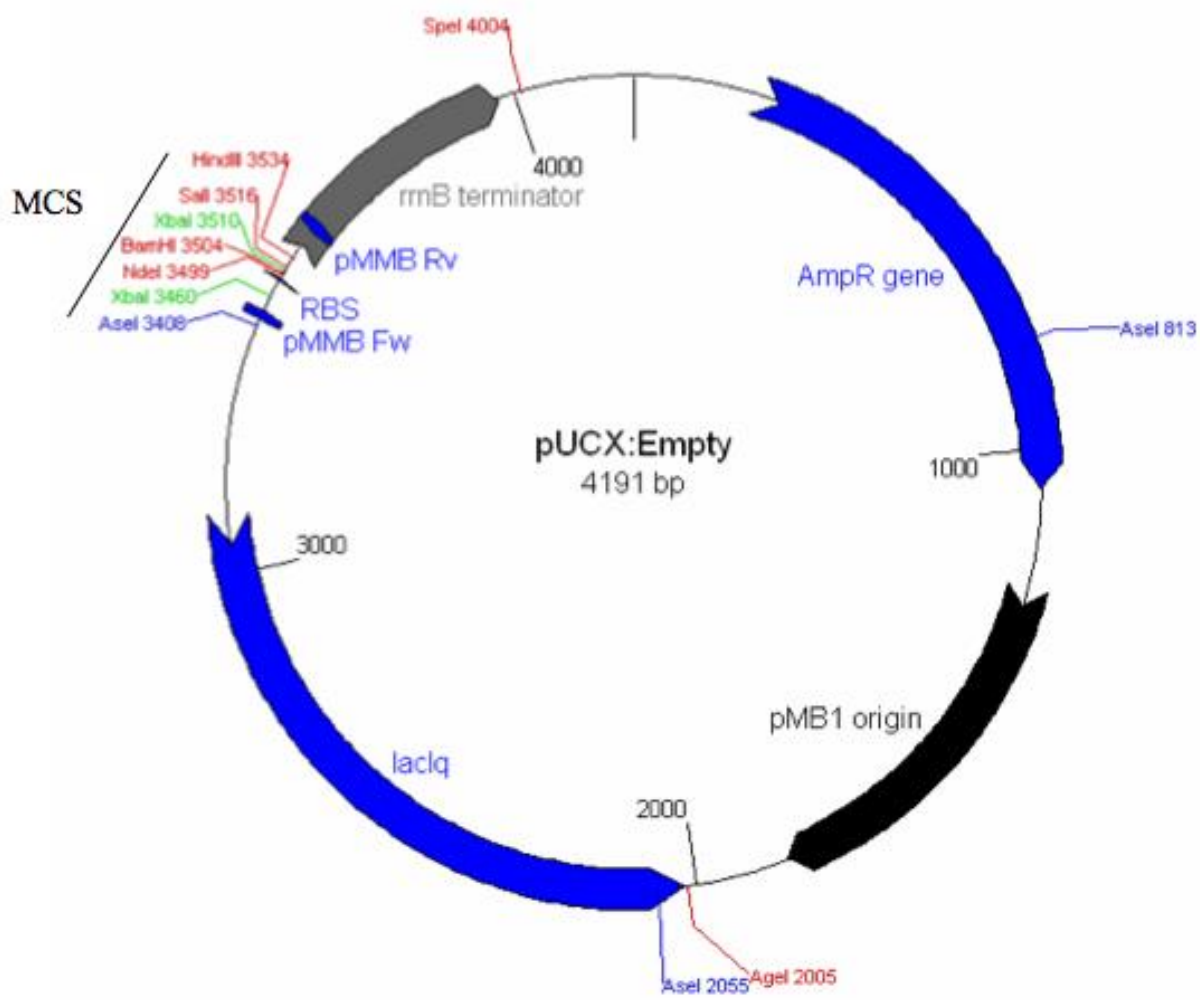

Figure 3.1 Map of pUCX expression plasmid. This plasmid was created by Dr Gareth Prosser by combining parts of commercially available plasmids pMMB and pUC19 to give a plasmid that exhibited improved expression of nitroreductases inserted into the multiple cloning site (MCS) (Prosser et al., 2010). The empty plasmid was used as a control in library screening.

The library was specifically designed to identify nitroreductases which were capable of reduction of nitro-quenched prodrugs. A core library of 58 nitroreductases (table 3.1) was established to test activity with the prodrugs CB1954 and PR104A (Prosser et al., 2013). It was discovered that nitroreductases that are members of the NfsA or NfsB families were consistently the most active (19 of the 20 most active enzymes) with each prodrug (Prosser et al., 2013). This general trend has also been seen across a wide range of other nitro-aromatic substrates (Dr David Ackerley, personal communication).

\subsubsection{Aims}

The key aims of the research described in this chapter were to: i) Measure the activation profiles of the 16 different nil bystander antibiotics with members of the 58 nitroreductase core library, identifying nitroreductases with higher activation of metronidazole (and other promising prodrugs) than NfsB_Ec, and identifying pairs of 
nil bystander antibiotics/ nitroreductases which show opposing activation profiles; ii) Translate the results observed in bacterial cells into human cell lines for proof of principle of use in eukaryotic models; and (iii) Explore the potential of niclosamide to be used in a functional screening system for the discovery of new nitroreductases.

\subsection{Results}

\subsubsection{Identification of nitroreductases with a superior activation profile with metronidazole than NfsB_Ec}

As noted above, contemporary studies in mammalian models in developmental and regenerative biology are exploring the use of nitroreductases paired with nil bystander antibiotics for temporally and spatially controlled targeted cell ablation. These studies have focused on the combination of metronidazole and to date have exclusively used the nitroreductase NfsB_Ec. However, in previous pilot screens of a subset of the 58 nitroreductase library in the Ackerley lab, NfsB_Ec didn't register as a nitroreductase with good efficacy for metronidazole. In order to more rigorously quantify the relative activity of the different nitroreductases in the 58 nitroreductase library, this library was screened with metronidazole by growth inhibition assay (Section 2.9.2). Growth inhibition assays allow the evaluation of the potency of a compound in given cell population. For example, higher levels of cell death at a particular concentration of indicate higher reduction efficiency by the nitroreductase present, subsequently causing a higher growth inhibition in these populations than in populations which the nitroreductases ability to activate metronidazole is less efficient. Growth inhibition assays are relatively high throughput and reproducible, giving reliable data on which to base further experiments. The results of the metronidazole growth inhibition screening are illustrated in Figure 3.2. 


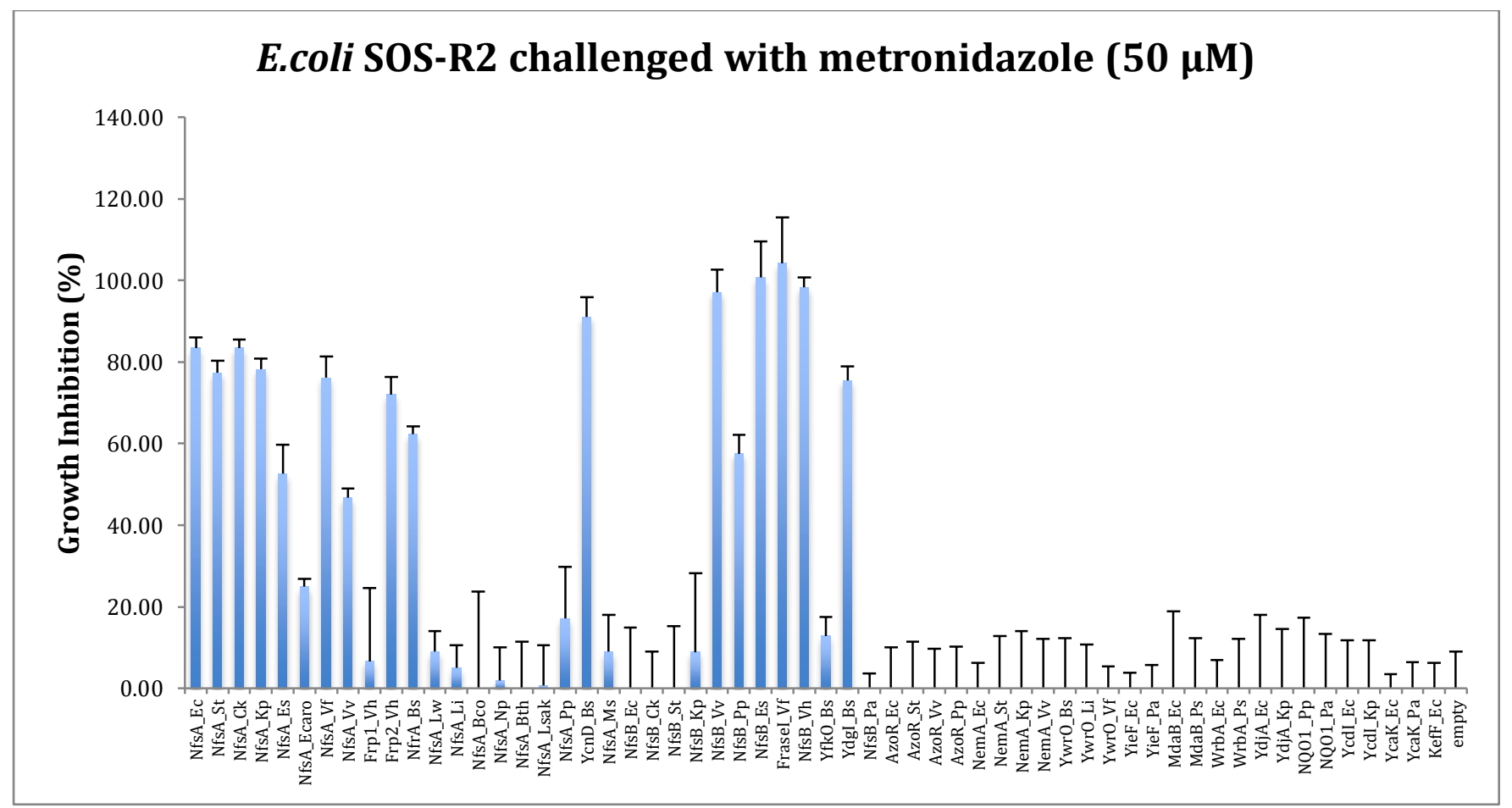

Figure 3.2 Metabolism of metronidazole by members of the 58 nitroreductase over-expression library as measured by growth inhibition assay. Turbidity $\left(\mathrm{OD}_{600}\right)$ of nitroreductase over-expressing E. coli cultures was recorded directly before and after $4 \mathrm{~h}$ incubation with $50 \mu \mathrm{M}$ metronidazole. Growth Inhibition is expressed as the percentage decrease in $\mathrm{OD}_{600}$ of challenged cells relative to unchallenged control cells for each strain post-incubation. Data are the average of 5 independent assays and the error bars indicate \pm 1 standard deviation. The bars corresponding to the NfsA and NfsB family members are as marked, and the arrow indicates NfsB_Ec. The empty plasmid control is indicated by "Empty", at the far right of the graph.

As can be seen in figure 3.2, the initial screening in bacterial cells showed that many other nitroreductases were capable of activating metronidazole at far lower concentrations. The nitroreductase with the highest activation profiles with metronidazole were from the NfsB family; NfsB_Vh, NfsB_Vv, NfsB_Es, and the NfsB like FraseI_Vf. More quantitative $\mathrm{IC}_{50}$ assays were carried out with E. coli strains over-expressing these nitroreductases as well as NfsB_Ec, NfsA_Ec and pUCX empty plasmid control (figure 3.3). 


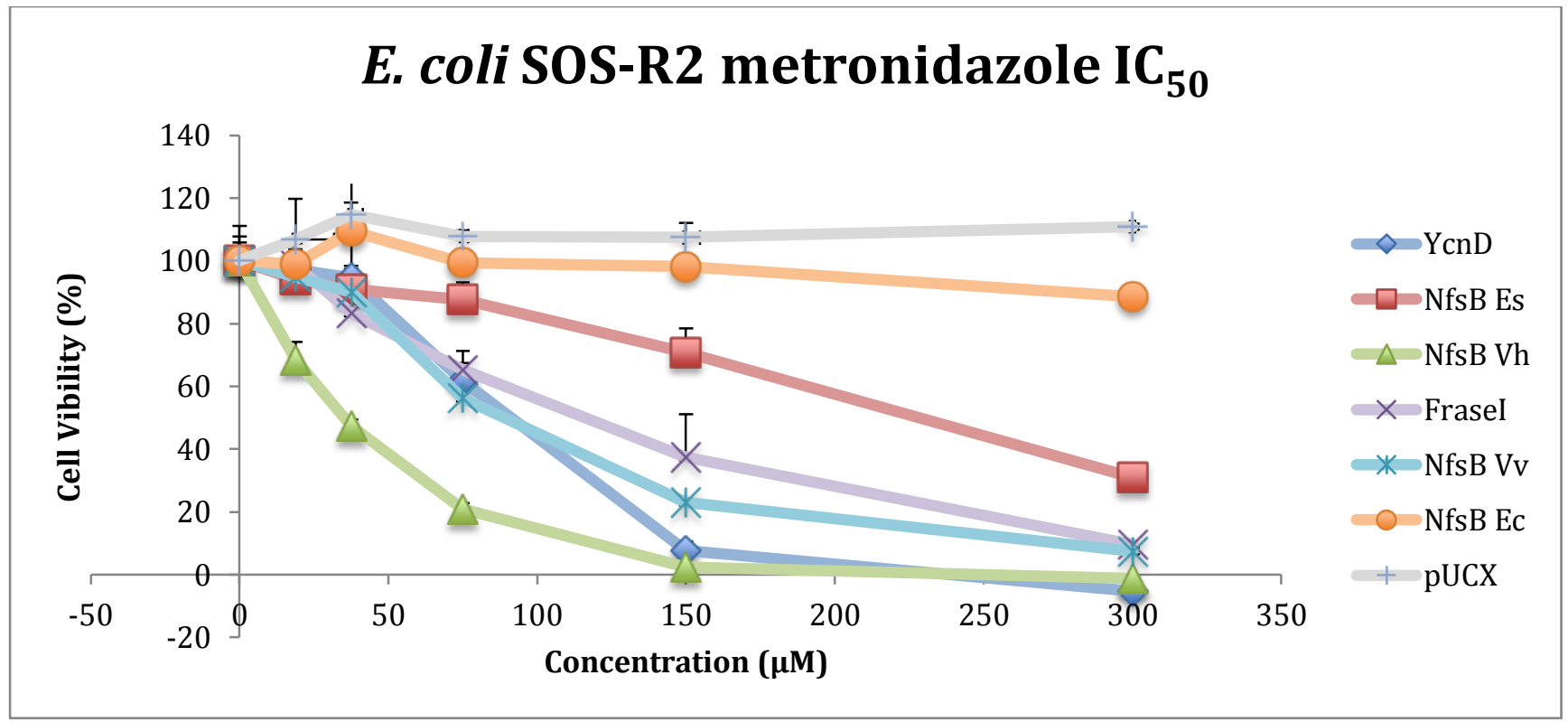

Figure 3.3 Metabolism of metronidazole by 6 nitroreductase enzymes from the 58 nitroreductase enzyme overexpression library over a range of concentrations as measured by $\mathbf{I C}_{\mathbf{5 0}}$ analysis. Turbidity $\left(\mathrm{OD}_{600}\right)$ of nitroreductase over-expressing E. coli cultures was recorded directly before and after $4 \mathrm{~h}$ incubation with 5 different metronidazole concentrations $(18.75 \mu \mathrm{M}, 37.5 \mu \mathrm{M}, 75 \mu \mathrm{M}, 150 \mu \mathrm{M} 300 \mu \mathrm{M})$. Cell viability is expressed as the percentage decrease in $\mathrm{OD}_{600}$ of challenged cells relative to unchallenged control cells for each strain post-incubation. Data are the average of 3 independent assays and the error bars indicate \pm 1 standard deviation.

Table 3.1 IC 50 results of 6 nitroreductase enzymes from the 58 nitroreductase enzyme over-expression library

This table shows the $\mathrm{IC}_{50}$ values shown graphically in figure 3.3 as calculated by the statistical analysis software Prism $^{\odot}$.

\begin{tabular}{|c|c|}
\hline Nitroreductase & $\mathrm{IC}_{50}$ Value $(\mu \mathrm{M})$ \\
\hline YcnD & 76.62 \\
\hline NfsB Es & 431.2 \\
\hline NfsB Vh & 47.48 \\
\hline FraseI & 169.2 \\
\hline NfsB Vv & 90.12 \\
\hline NfsB Ec & 928.1 \\
\hline
\end{tabular}

From the results, we can conclude that all of the nitroreductases identified in the initial full-library screen as having substantially greater activity with metronidazole than NfsB_Ec were confirmed by the $\mathrm{IC}_{50}$ analysis. NfsB_Vh showed the best activity. We have previously observed that nitroreductase activity seen in bacterial cells isn't always translated into human cell lines. Fortunately, as part of a different study NfsB_Vh had previously been transfected into the human colon carcinoma cell line HCT-116 by Dr Horvat, so this stably transfected cell line was able to be used in initial experiments for metronidazole activity in human cell lines. 
Cells were seeded into T25 and treated with $5 \mu \mathrm{M}$ drug - this drug concentration was based on previous MTS assays carried out by Dr Horvat, which indicated that after a $48 \mathrm{~h}$ period $5 \mu \mathrm{M}$ metronidazole was an optimal concentration to induce complete cell death in human cell lines sensitive to metronidazole.

These experiments in HCT-116 NfsB_Vh showed that a low concentration of metronidazole was capable of inducing cell death in these cell lines as can be seen in figure 3.4. A HEK293: NfsB_Ec cell line was generated as per described in section 3.2.5. to compare the effect of metronidazole on human cells when NfsB_Ec was present. As can be seen in figure 3.5, metronidazole appears to have no effect on human cell lines containing the nitroreductase NfsB_Ec. This demonstrates that $\mathrm{NfsB}$ _Vh is far superior at activating metronidazole than NfsB_Ec in human cells, suggesting that it would be an ideal candidate for further experimental work in eukaryotic model organisms. 


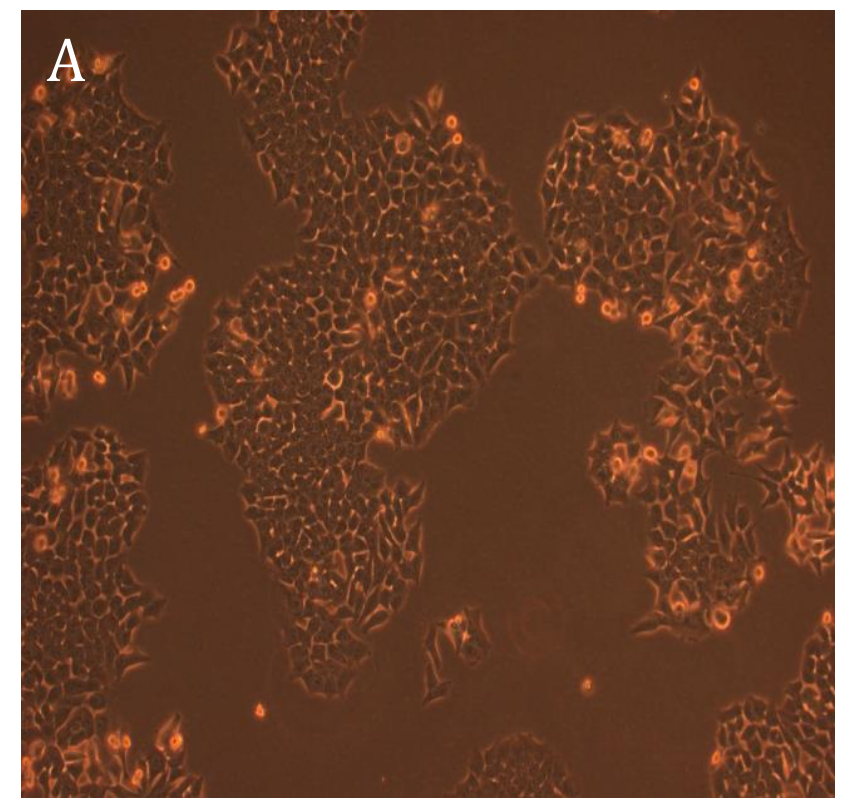

Figure 3.4 Metronidazole challenged HCT116: NfsB Vh cells. Cells were seeded at a density of 250,000 into T25 flasks and left to grow overnight to approximately $80 \%$ confluency. $5 \mu \mathrm{M}$ metronidazole was added to the cells and they were left for $48 \mathrm{~h}$. Bright field images of the cell population were taken at $0 \mathrm{~h}$ and $48 \mathrm{~h}$ with an Olympus IX51 microscope using the 20x lens. A. HCT-116: NfsB Vh cells ( 0 h) B. HCT-116 $\mathrm{NfsB}$ Vh cells challenged with $5 \mu \mathrm{M}$ metronidazole $48 \mathrm{~h}$. After 48 hours in the presence of a low concentration of metronidazole, the cells look unhealthy as the cell morphology has changed and population has decreased dramatically compared to when the population had not been in the presence of metronidazole.

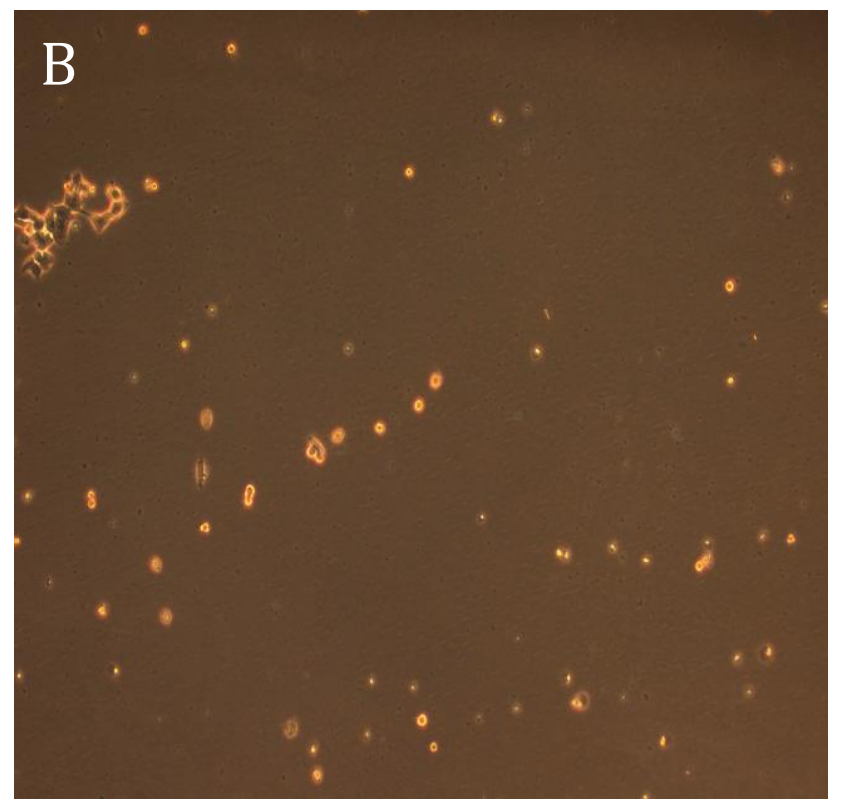




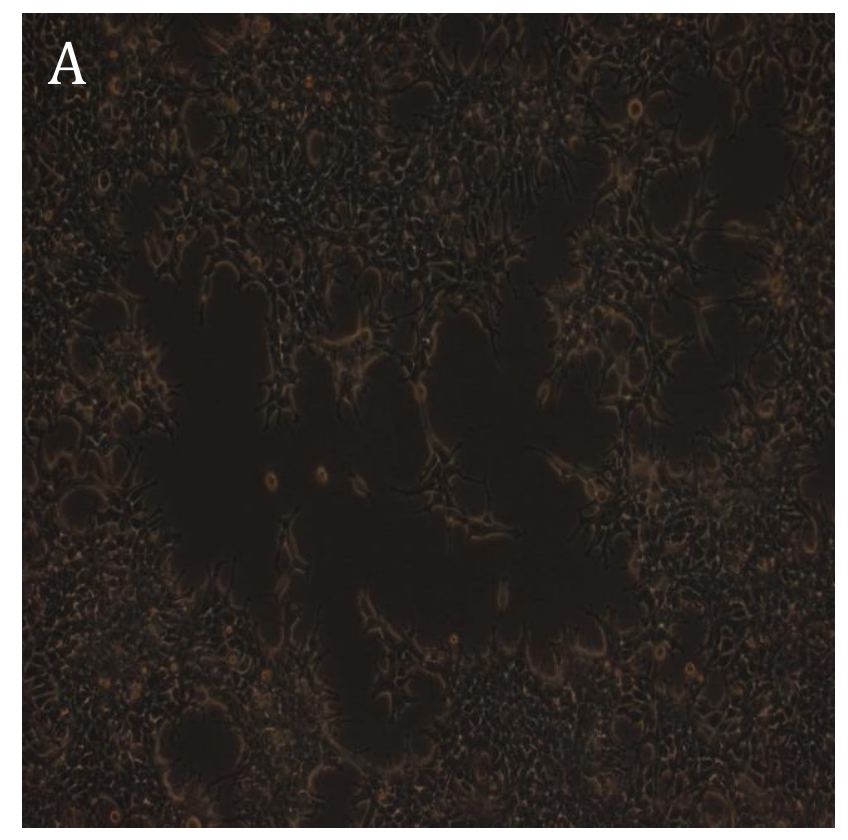

Figure 3.5 Metronidazole challenged HEK293: NfsB_Ec cells. Cells were seeded at a density of 250,000 into T25 flasks and left to grow overnight to approximately $80 \%$ confluency. $5 \mu \mathrm{M}$ metronidazole was added to the cells and they were left for $48 \mathrm{~h}$. Bright field images of the cell population were taken at $0 \mathrm{~h}$ and $48 \mathrm{~h}$ with an Olympus IX51 microscope using the 20x lens. A. HEK293: NfsB_Ec (0 h) B. HEK293:

NfsB_Ec cells challenged with $5 \mu \mathrm{M}$ metronidazole $48 \mathrm{~h}$. After 48 hours in the presence of a low concentration of metronidazole, the cells look healthy as the cell morphology is unchanged and cell population appears to have increased compared to $0 \mathrm{~h}$, suggesting at concentrations this low, NfsB_Ec is incapable of activating metronidazole in human cell line HEK293.

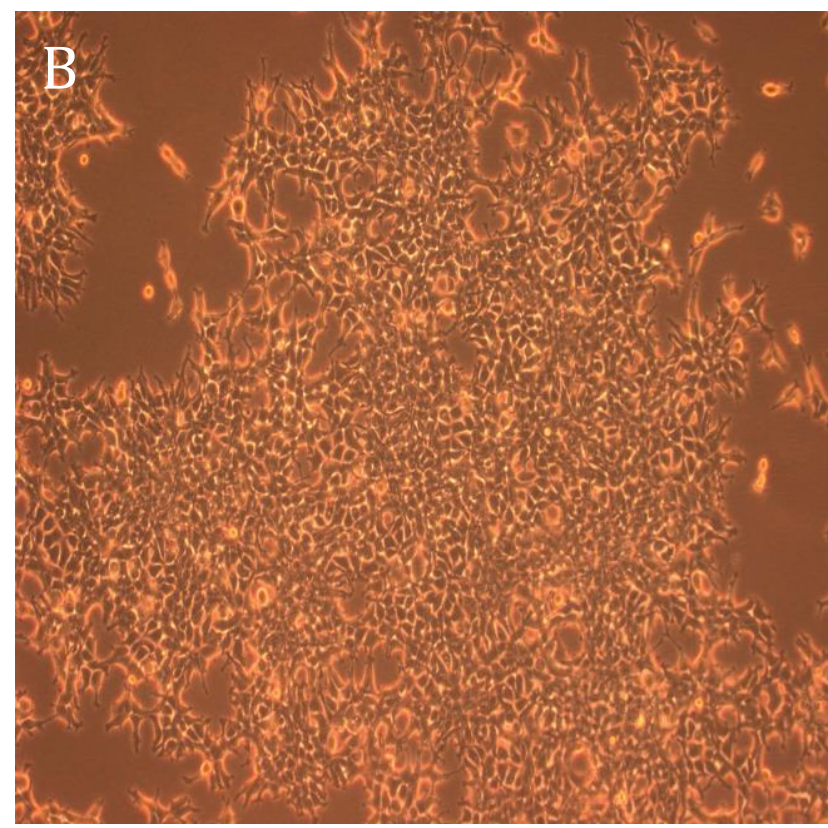

\subsubsection{Growth inhibition assays}

As an alternative approach to identifying a nitroreductase with higher activity with metronidazole than NfsB_Ec, we explored the possibility of employing a different nil bystander antibiotic for use in the ablation of specific cell types in developmental biology model organisms. Growth inhibition assays as detailed in 2.9.2 were carried out on the 58 nitroreductase core library with all 16 of the nil bystander antibiotics as listed in table 3.1. Each assay was carried out at least 5 times to guarantee statistical 
significance, as these results were to provide the basis of all further experimental research in this thesis.

The percentage of cell death for each nil bystander antibiotic/ nitroreductase combination was determined by calculating the percentage of cell death after 4 hours of challenge with the nil bystander antibiotic compared to unchallenged control cells for each strain after 4 hours. Graphs of each of these nil bystander antibiotic/ 58 nitroreductase library screens were generated by averaging the percentage of cell death observed and adding error bars (+/- 1 standard deviation).

To provide an easily interpreted summary of which nil bystander antibiotic/ nitroreductase combinations were active or not active, arbitrary cut off points were set to denote high activity, some activity, or no activity. These points were $75-100 \%$ growth inhibition, 30-74\% growth inhibition and 0-29\% growth inhibition respectively. The numerical data from the screening was collated into a table colourcoded heat-map to denote these percentage cutoffs - green high activity, orange some activity, or red no activity. The results of this screening can be seen below in the heatmap (figure 3.6). 


\begin{tabular}{|c|c|c|c|c|c|c|c|c|c|c|c|c|c|c|c|c|}
\hline & $2 \mathrm{M} 5 \mathrm{NI}$ & $4 \mathrm{NI}$ & DTZ & EF5 & FMISO & FUR & MTZ & MISO & NB2NI & NCS & NFX & NFN & NFZ & ORN & TIN & RNZ \\
\hline Nffs_Ec & 17.42 & 72.63 & 14.99 & 57.24 & 79.51 & 83.18 & 83.45 & 85.63 & 107.11 & 24.84 & 80.14 & 84.76 & 91.08 & 81.70 & 93.17 & 108.47 \\
\hline NfsA_St & 28.65 & 70.98 & 19.67 & 105.40 & 100.16 & 82.00 & 77.37 & 79.03 & 106.71 & 29.08 & 77.69 & 88.22 & 93.04 & 78.66 & 72.39 & 99.39 \\
\hline NfsA_Ck & 45.64 & 71.02 & 5.37 & 67.59 & 90.63 & 76.90 & 83.44 & 79.25 & 101.20 & 37.46 & 78.91 & 57.85 & 88.85 & 83.47 & 93.44 & 96.22 \\
\hline NfsA_Kp & 26.46 & 53.43 & -2.22 & 32.16 & 38.20 & 86.54 & 78.20 & 76.29 & 98.33 & 84.33 & 74.03 & 64.35 & 93.65 & 79.22 & 81.99 & 86.21 \\
\hline NfsA_Es & 36.06 & 58.01 & 3.43 & 84.75 & 87.16 & 85.75 & 52.61 & 86.73 & 104.87 & 110.09 & 47.33 & 55.28 & 94.90 & 39.99 & 19.95 & 73.10 \\
\hline $\begin{array}{l}\text { NfsA_Ecar } \\
\text { o }\end{array}$ & 31.20 & 40.29 & 9.82 & 91.47 & 88.50 & 89.83 & 24.94 & 82.56 & 104.93 & 102.18 & 74.10 & 77.85 & 97.16 & 56.68 & 71.82 & 67.59 \\
\hline NfsA_Vf & 52.01 & 69.22 & 45.55 & 31.44 & 76.37 & 81.82 & 76.13 & 95.35 & 111.16 & 2.12 & 72.98 & 76.98 & 77.74 & 85.68 & 94.49 & 103.91 \\
\hline NfsA_Vv & 61.24 & 66.20 & 0.00 & 20.93 & 66.59 & 90.09 & 46.83 & 90.77 & 108.13 & 0.00 & 73.73 & 58.75 & 95.48 & 85.98 & 86.54 & 98.44 \\
\hline Frp1_Vh & 0.00 & 14.72 & 18.18 & 68.32 & 71.88 & 57.63 & 6.67 & 57.91 & 86.33 & 103.36 & 35.02 & 66.31 & 36.24 & 50.06 & 56.81 & 90.71 \\
\hline Frp2_Vh & 65.64 & 64.71 & 41.12 & 39.16 & 79.05 & 86.46 & 72.07 & 85.04 & 103.95 & 37.33 & 62.46 & 87.71 & 99.01 & 84.02 & 92.78 & 104.35 \\
\hline NfrA_Bs & 41.43 & 74.51 & 19.26 & 31.14 & 65.85 & 94.99 & 62.31 & 93.82 & 102.28 & 46.74 & 83.98 & 83.54 & 103.29 & 98.23 & 93.81 & 103.85 \\
\hline NfsA_Lw & 29.81 & 32.81 & 0.00 & 57.84 & 46.26 & 89.72 & 8.99 & 84.97 & 96.86 & 106.23 & 73.23 & 70.90 & 101.81 & 43.56 & 52.60 & 64.64 \\
\hline NfsA_Li & 7.79 & 11.96 & 23.15 & 22.87 & 34.90 & 72.62 & 5.08 & 80.04 & 87.90 & 98.14 & 51.40 & 65.43 & 71.39 & 71.15 & 89.10 & 85.18 \\
\hline NfsA_Bco & 3.23 & 61.14 & 0.00 & 45.33 & 85.82 & 82.90 & 0.00 & 69.47 & 91.81 & 107.48 & 84.92 & 69.92 & 79.31 & 2.16 & 9.44 & 75.52 \\
\hline NfsA_Np & 12.22 & 9.73 & 0.00 & 76.81 & 76.80 & 71.92 & 1.99 & 75.29 & 72.21 & 108.90 & 56.92 & 72.86 & 71.13 & 24.65 & 28.30 & 30.83 \\
\hline NfsA_Bth & 6.11 & 70.15 & 0.00 & 47.65 & 75.18 & 47.70 & 0.00 & 88.37 & 96.68 & 56.94 & 72.31 & 33.88 & 97.04 & 18.71 & 0.00 & 96.02 \\
\hline NfsA_Lsak & 4.91 & 17.27 & 7.06 & 41.51 & 55.67 & 82.78 & 0.64 & 51.17 & 52.18 & 108.67 & 63.87 & 69.49 & 87.75 & 0.72 & 18.41 & 27.28 \\
\hline NfsA_Pp & 22.73 & 14.66 & 6.65 & 59.23 & 78.30 & 75.63 & 17.11 & 83.06 & 100.75 & 39.04 & 81.82 & 77.86 & 80.60 & 75.38 & 87.64 & 77.85 \\
\hline YcnD_Bs & 30.83 & 94.85 & 98.04 & 13.85 & 95.83 & 54.33 & 91.08 & 110.13 & 43.03 & 7.49 & 61.45 & 37.76 & 93.60 & 97.68 & 54.59 & 108.52 \\
\hline NfsA_Ms & 2.90 & 63.59 & 6.18 & 11.53 & 24.10 & 75.41 & 8.98 & 32.51 & 37.50 & 108.30 & 47.74 & 69.54 & 48.93 & 11.80 & 17.51 & 26.55 \\
\hline NfsB_Ec & 0.00 & 13.61 & 10.67 & 3.00 & 4.30 & 62.98 & 0.00 & 14.79 & 445.01 & 24.73 & 65.46 & 75.98 & 55.55 & 10.02 & 19.36 & 63.58 \\
\hline NfsB_Ck & 0.00 & 17.16 & 3.59 & 2.23 & 5.99 & 89.31 & 0.00 & 24.06 & 42.30 & 24.63 & 63.88 & 70.12 & 54.69 & 11.86 & 13.95 & 102.25 \\
\hline NfsB_St & 10.86 & 14.56 & 32.82 & 0.99 & 3.22 & 82.32 & 0.00 & 10.84 & 15.47 & 26.57 & 75.08 & 58.06 & 54.20 & 25.82 & 36.84 & 115.15 \\
\hline NfsB_Kp & 5.96 & 14.58 & 11.95 & 0.00 & 1.65 & 78.40 & 8.90 & 53.14 & 53.97 & 14.07 & 69.33 & 62.29 & 44.73 & 58.98 & 58.78 & 107.59 \\
\hline $\mathrm{NfsB}_{-} \mathrm{Vv}$ & 12.41 & 30.05 & 74.31 & 0.00 & 5.85 & 93.42 & 97.07 & 85.90 & 8.36 & 0.00 & 73.49 & 65.81 & 67.77 & 77.53 & 28.77 & 119.35 \\
\hline NfsB_Pp & 0.00 & 47.60 & 35.74 & 0.00 & 30.01 & 89.63 & 57.56 & 44.44 & 40.62 & 70.57 & 77.62 & 55.55 & 79.08 & 18.91 & 7.84 & 110.65 \\
\hline NfsB_Es & 0.00 & 0.37 & 87.01 & 0.94 & 0.03 & 77.37 & 100.79 & 69.23 & 43.40 & 22.75 & 62.88 & 55.87 & 66.69 & 85.53 & 87.19 & 108.15 \\
\hline FraseI_Vf & 170.52 & 6.57 & 99.16 & 0.33 & 15.37 & 89.67 & 104.22 & 80.84 & 54.29 & 22.84 & 67.91 & 60.57 & 34.94 & 86.34 & 100.54 & 104.16 \\
\hline NfsB_Vh & 0.00 & 4.80 & 97.66 & 7.17 & 11.57 & 71.97 & 98.26 & 65.68 & 15.45 & 57.01 & 72.47 & 49.96 & 56.84 & 93.24 & 70.08 & 113.15 \\
\hline YfkO_Bs & 0.00 & 1.96 & 33.02 & 14.26 & 12.45 & 79.12 & 12.85 & 67.76 & 70.40 & 26.55 & 86.15 & 60.56 & 99.23 & 24.44 & 49.75 & 107.74 \\
\hline YdgI_Bs & 0.00 & 15.91 & 36.74 & 0.78 & 11.06 & 84.04 & 75.45 & 94.03 & 27.38 & 95.10 & 65.60 & 71.91 & 63.97 & 95.11 & 105.66 & 107.91 \\
\hline NfsB_Pa & 0.00 & 0.00 & 6.71 & 0.00 & 0.00 & 82.82 & 0.00 & 0.00 & 20.90 & 106.37 & 57.26 & 52.52 & 58.25 & 8.71 & 0.00 & 99.22 \\
\hline AzoR_Ec & 0.00 & 0.00 & 6.26 & 0.00 & 0.00 & 74.53 & 0.00 & 6.58 & 10.04 & 9.87 & 47.08 & 26.22 & 45.76 & 0.00 & 9.79 & 24.42 \\
\hline
\end{tabular}




\begin{tabular}{|c|c|c|c|c|c|c|c|c|c|c|c|c|c|c|c|c|}
\hline AzoR_St & 0.00 & 0.00 & 8.20 & 0.00 & 0.00 & 80.74 & 0.00 & 7.83 & 17.47 & 7.64 & 67.29 & 35.40 & 50.54 & 3.59 & 0.00 & 43.46 \\
\hline AzoR_Vv & 7.46 & 0.00 & 3.12 & 0.00 & 0.00 & 73.88 & 0.00 & 6.25 & 10.34 & 52.51 & 64.46 & 27.40 & 50.85 & 20.08 & 0.00 & 16.30 \\
\hline AzoR_Pp & 0.00 & 0.00 & 3.30 & 0.00 & 0.00 & 77.79 & 0.00 & 6.43 & 1.41 & 76.48 & 58.33 & 31.07 & 45.25 & 0.00 & 0.00 & 13.60 \\
\hline NemA_Ec & 0.00 & 0.00 & 5.62 & 0.00 & 0.00 & 76.80 & 0.00 & 17.88 & 22.74 & 108.44 & 34.62 & 32.14 & 34.26 & 17.29 & 20.55 & 12.16 \\
\hline NemA_St & 0.00 & 0.00 & 0.00 & 0.00 & 0.00 & 54.22 & 0.00 & 11.67 & 16.04 & 111.36 & 7.17 & 29.03 & 15.73 & 4.77 & 3.26 & 0.00 \\
\hline NemA_Kp & 0.00 & 0.00 & 3.03 & 0.00 & 0.00 & 55.45 & 0.00 & 26.60 & 0.00 & 112.32 & 13.42 & 32.08 & 42.98 & 10.39 & 3.49 & 0.66 \\
\hline NemA_Vv & 0.00 & 0.00 & 6.77 & 0.00 & 0.00 & 78.81 & 0.00 & 13.34 & 28.39 & 115.46 & 35.78 & 28.85 & 61.01 & 11.10 & 5.87 & 13.91 \\
\hline $\begin{array}{l}\text { YwrO_Bs } \\
\end{array}$ & 0.36 & 0.00 & 0.00 & 0.00 & 0.00 & 38.88 & 0.00 & 11.96 & 17.17 & 112.04 & 4.41 & 16.97 & 26.04 & 13.71 & 0.00 & 0.00 \\
\hline $\begin{array}{l}\text { YwrO_Li } \\
\end{array}$ & 47.69 & 0.00 & 0.00 & 0.00 & 0.00 & 34.98 & 0.00 & 12.49 & 16.95 & 111.98 & 2.34 & 26.95 & 6.80 & 1.20 & 0.00 & 0.00 \\
\hline YwrO_Vf & 0.00 & 0.00 & 0.00 & 0.00 & 0.00 & 49.54 & 0.00 & 2.49 & 19.34 & 110.22 & 0.00 & 28.26 & 12.50 & 0.00 & 0.00 & 0.00 \\
\hline YieF_Ec & 4.33 & 9.60 & 0.00 & 0.00 & 0.00 & 53.97 & 0.00 & 0.00 & 49.43 & 108.27 & 0.00 & 23.11 & 18.26 & 8.94 & 0.00 & 5.74 \\
\hline YieF_Pa & 0.00 & 8.55 & 1.56 & 0.00 & 1.46 & 40.90 & 0.00 & 5.13 & 52.73 & 117.58 & 0.00 & 26.88 & 21.23 & 0.00 & 0.00 & 21.08 \\
\hline MdaB_Ec & 0.00 & 13.52 & 41.00 & 0.00 & 0.00 & 62.61 & 0.00 & 13.31 & 43.31 & 118.95 & 16.35 & 24.94 & 19.32 & 14.69 & 3.96 & 44.92 \\
\hline MdaB_Ps & 0.00 & 0.00 & 0.00 & 0.00 & 0.00 & 43.67 & 0.00 & 4.50 & 18.56 & 116.50 & 14.78 & 7.96 & 62.08 & 0.00 & 0.00 & 0.00 \\
\hline WrbA_Ec & 0.00 & 0.00 & 6.23 & 0.00 & 0.00 & 22.20 & 0.00 & 15.47 & 20.07 & 113.62 & 0.00 & 18.80 & 18.79 & 0.00 & 0.00 & 4.22 \\
\hline WrbA_Ps & 0.00 & 0.33 & 0.00 & 0.00 & 0.00 & 52.74 & 0.00 & 2.33 & 17.06 & 113.16 & 2.07 & 24.06 & 74.30 & 0.00 & 0.00 & 0.00 \\
\hline YdjA_Ec & 0.00 & 3.29 & 0.00 & 0.00 & 7.95 & 46.91 & 0.00 & 3.57 & 19.98 & 114.79 & 0.00 & 0.56 & 17.41 & 0.00 & 0.00 & 0.13 \\
\hline $\begin{array}{l}\text { YdjA_Kp } \\
\end{array}$ & 0.00 & 0.00 & 0.00 & 0.00 & 8.04 & 30.62 & 0.00 & 0.00 & 15.25 & 115.23 & 0.00 & 12.89 & 11.91 & 0.11 & 0.00 & 0.00 \\
\hline NQO1_Pp & 0.00 & 3.33 & 0.00 & 0.00 & 11.81 & 27.43 & 0.00 & 0.00 & 29.10 & 113.58 & 0.00 & 0.00 & 23.00 & 0.00 & 0.00 & 5.15 \\
\hline NQO1_Pa & 0.00 & 0.00 & 0.00 & 0.00 & 0.00 & 10.80 & 0.00 & 0.00 & 20.44 & 115.86 & 0.00 & 10.66 & 21.71 & 3.54 & 19.63 & 0.00 \\
\hline YcdI_Ec & 0.00 & 18.76 & 0.00 & 0.00 & 0.00 & 19.60 & 0.00 & 0.00 & 30.68 & 115.82 & 9.06 & 28.78 & 13.15 & 0.00 & 33.84 & 0.00 \\
\hline YcdI_Kp & 0.00 & 0.00 & 0.00 & 0.00 & 0.00 & 46.20 & 0.00 & 0.00 & 46.67 & 108.63 & 0.00 & 7.95 & 7.67 & 0.00 & 0.00 & 0.00 \\
\hline YcaK_Ec & 0.00 & 0.00 & 0.00 & 0.00 & 0.00 & 68.12 & 0.00 & 0.00 & 36.79 & 109.43 & 16.50 & 1.30 & 0.00 & 9.10 & 0.00 & 4.36 \\
\hline YcaK_Pa & 7.03 & 4.48 & 0.00 & 0.00 & 9.42 & 26.37 & 0.00 & 0.00 & 22.42 & 119.87 & 0.00 & 19.60 & 35.02 & 0.00 & 0.00 & 0.00 \\
\hline KefF_Ec & 0.00 & 0.00 & 0.00 & 0.00 & 0.00 & 45.28 & 0.00 & 0.00 & 29.58 & 111.13 & 3.63 & 15.82 & 24.81 & 0.38 & 0.00 & 6.06 \\
\hline empty & 0.00 & 0.00 & 0.00 & 0.00 & 0.00 & 25.10 & 0.00 & 6.67 & 19.10 & 110.95 & 0.00 & 12.34 & 0.00 & 0.00 & 0.00 & 0.00 \\
\hline
\end{tabular}

Figure 3.6. Colour coded heat-map summarising activation profiles of nitroreductase/ nil bystander antibiotic combinations. The heat-map was generated from growth inhibition averages from 5 independent assays. Growth inhibition is expressed as the percentage decrease in $\mathrm{OD}_{600}$ of challenged cells relative to unchallenged control cells for each strain postincubation. Turbidity $\left(\mathrm{OD}_{600}\right)$ of nitroreductase over-expressing E. coli cultures was recorded directly before and after $4 \mathrm{~h}$ incubation with nil bystander antibiotic. In this heat-map, the green squares denote nitroreductase enzymes that have high activity with the given nil bystander antibiotic, orange some activity, and red no activity. These activity margins are deduced from growth inhibition assays giving either 75-100\%, 30-74\%, or 0-29\% cell death respectively (percentages given within each cell to 2dp). (2M5NI) 2-Methyl-5-Nitroimidazole (200 $\mu \mathrm{M}$ ), (4NI) 4-Nitroimidazole $(25 \mu \mathrm{M})$, (DTZ) Dimetridazole $(25 \mu \mathrm{M})$, EF5 (130 $\mu \mathrm{M})$, (FMISO) F-Misonidazole (130 $\mu \mathrm{M})$, (FUR) Furazolidone (45 $\mu \mathrm{M})$, (MTZ) Metronidazole (50 $\mu \mathrm{M})$, (MISO)

Misonidazole $(300 \mu \mathrm{M})$, (NB2NI) $N$-Benznidazole $(40 \mu \mathrm{M})$, (NCS) Niclosamide (2.5 $\mu \mathrm{M})$, (NFX) Nifurtimox $(75 \mu \mathrm{M})$, (NFN) Nitrofurantoin $(24 \mu \mathrm{M})$, (NFZ) Nitrofurazone (60 $\mu \mathrm{M})$, (ORN) Ornidazole (75 $\mu \mathrm{M})$, (TIN) Tinidazole $(75 \mu \mathrm{M})$, (RNZ) Ronidazole $(150 \mu \mathrm{M})$. 


\subsubsection{Identification of pairs of nitroreductases / nil bystander antibiotics with opposing activation profiles}

The heat-map in figure 3.6 allows rapid identification of the nitroreductases that are most active with individual nil bystander antibiotics. It also revealed that some nitroreductase pairs have opposing activation profiles with pairs of nil bystander antibiotics. This lead to the proposal of implementing a dual targeted cell ablation system; simultaneously using one nitroreductase which is capable of activating a given nil bystander antibiotic but not a second nil bystander antibiotic, in partnership with a different nitroreductase which is capable of activating the second nil bystander antibiotic, but not the first one (as per the scheme in Figure 1.11). A system such as this would, by placing each nitroreductase under control of a distinct tissue-specific promoter in the same transgenic organism, enable the targeted ablation of two completely independent cell populations with temporal and spatial discretion.

The colour coded heat-map made it possible identify prospective pairs of nil bystander antibiotic/ nitroreductase combinations with opposing activation profiles.

Examples of promising combinations are summarised in Figures 3.7-3.10.

\begin{tabular}{|l|c|c|}
\cline { 2 - 3 } \multicolumn{1}{c|}{} & Tinidazole & Metronidazole \\
\hline NfsA_Ecaro & 97.16 & 24.94 \\
\hline NfsA_Li & 89.10 & 5.08 \\
\hline NfsA_Pp & 87.64 & 17.11 \\
\hline NfsB_Vv & 28.77 & 97.07 \\
\hline
\end{tabular}

Figure 3.7. Identification of nitroreductase enzymes that exhibit opposing activation profiles with tinidazole and metronidazole. Results summarised from heat-map, where green indicates high activity with that nil bystander antibiotic, while red indicates little to no activity. The numbers in each cell indicate the percentage of growth inhibition observed from the growth inhibition assays.

\begin{tabular}{|l|c|c|}
\cline { 2 - 3 } \multicolumn{1}{c|}{} & F-Miso & Metronidazole \\
\hline NfsA_Np & 76.80 & 1.99 \\
\hline NfsA_Bth & 75.18 & 0.00 \\
\hline NfsB_Vv & 5.85 & 97.07 \\
\hline
\end{tabular}

Figure 3.8 Identification of nitroreductase enzymes that exhibit opposing activation profiles with F-misonidazole and metronidazole. Results summarised from heat-map, where green indicates high activity with that nil bystander antibiotic, while red indicates little to no activity. The numbers in each cell indicate the percentage of growth inhibition observed from the growth inhibition assays. 


\begin{tabular}{|l|c|c|}
\cline { 2 - 3 } \multicolumn{1}{c|}{} & EF5 & Dimetridazole \\
\hline NfsA_Es & 84.75 & 3.43 \\
\hline NfsB_Vh & 7.17 & 97.66 \\
\hline
\end{tabular}

Figure 3.9 Identification of nitroreductase enzymes that exhibit opposing activation profiles with EF5 and dimetridazole. Results summarised from heat-map, where green indicates high activity with that nil bystander antibiotic, while red indicates little to no activity. The numbers in each cell indicate the percentage of growth inhibition observed from the growth inhibition assays.

\begin{tabular}{|l|c|c|}
\cline { 2 - 3 } \multicolumn{1}{c|}{} & EF5 & Metronidazole \\
\hline NfsA_Np & 76.81 & 1.99 \\
\hline YdgI_Bs & 0.78 & 75.45 \\
\hline NfsB_Vh & 7.17 & 98.26 \\
\hline
\end{tabular}

Figure 3.10 Identification of nitroreductase enzymes that exhibit opposing activation profiles with EF5/ and metronidazole. Results summarised from heat-map, where green indicates high activity with that nil bystander antibiotic, while red indicates little to no activity. The numbers in each cell indicate the percentage of growth inhibition observed from the growth inhibition assays.

$\mathrm{IC}_{50}$ analysis was subsequently undertaken on some promising pairs of nil bystander antibiotic/ nitroreductase combinations with opposing activation profiles. $\mathrm{IC}_{50}$ assays measure the effectiveness of a compound in inhibiting biological function, indicating the concentration of a compound required to inhibit growth by $50 \%$ and are a standard measure of drug potency. Focus was given to the nitroreductases that had opposing activities with tinidazole and metronidazole (figure 3.7) on the basis that these nil bystander antibiotics have both been well studied, are cheap and widely available, and all nitroreductases that were capable of activating tinidazole but not metronidazole were members of the NfsA family, whereas the nitroreductase that was found capable of activating metronidazole but not tinidazole was a member of the NfsB family these two families are distinctly different and the most well studied of the nitroreductase enzymes. IC50 analysis was undertaken with these nitroreductases and nil bystander antibiotics (figures 3.11-3.14). 


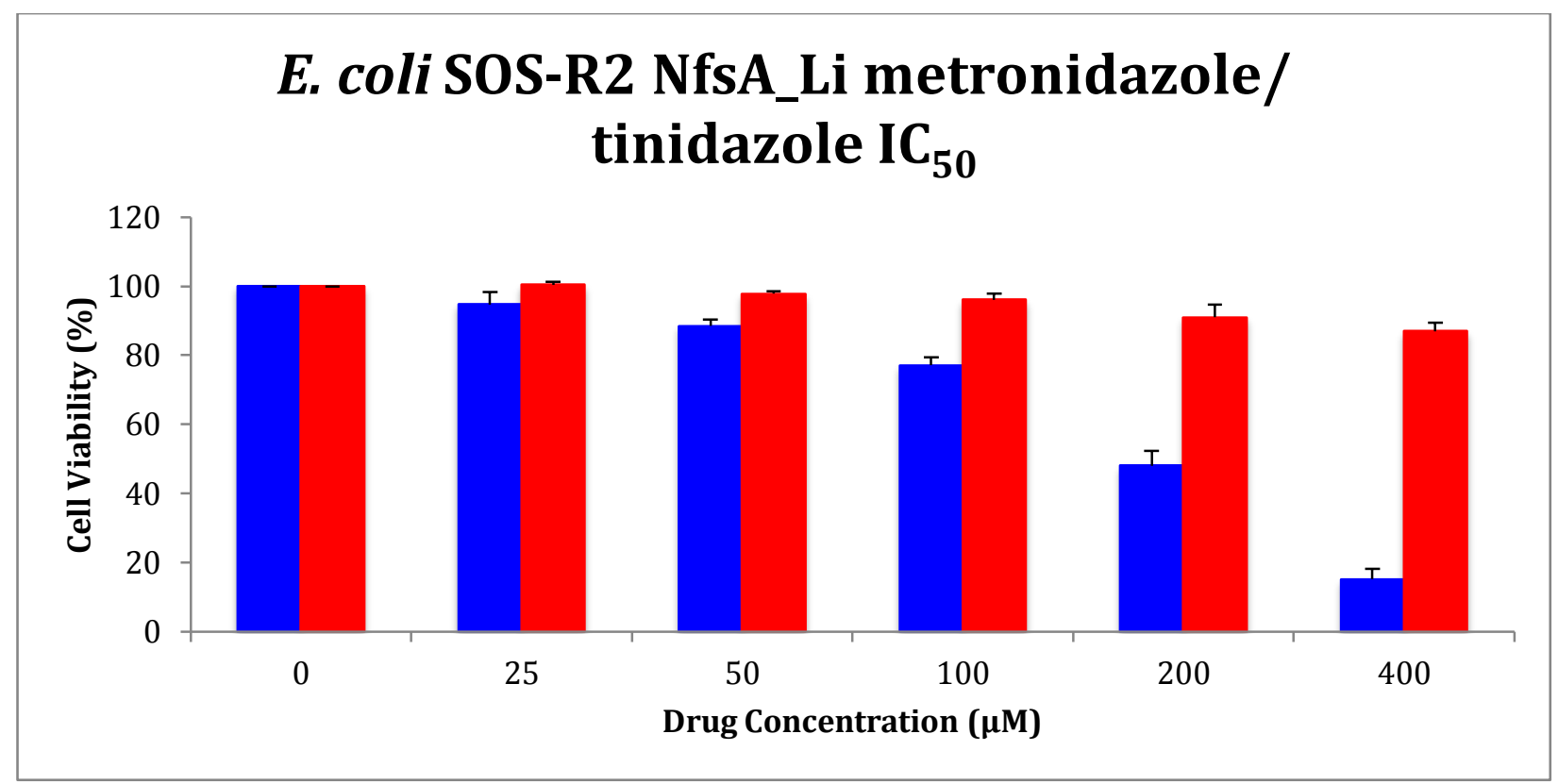

Figure 3.11 Metabolism of metronidazole and tinidazole by $\boldsymbol{E}$. coli cells over-expressing nitroreductase NfsA_Li over a range of concentrations as measured by $\mathbf{I C}_{\mathbf{5 0}}$ analysis. Turbidity $\left(\mathrm{OD}_{600}\right)$ of nitroreductase over-expressing $E$. coli cultures was recorded directly before and after $4 \mathrm{~h}$ incubation with 5 different nil bystander antibiotic concentrations $(25 \mu \mathrm{M}, 50 \mu \mathrm{M}, 100 \mu \mathrm{M}, 200 \mu \mathrm{M}$ $400 \mu \mathrm{M})$. Metronidazole is shown in blue, and tinidazole red. Cell viability is expressed as the percentage decrease in $\mathrm{OD}_{600}$ of challenged cells relative to unchallenged control cells for each strain post-incubation. Data are the average of 3 independent assays and the error bars indicate \pm 1 standard deviation. The $\mathrm{IC}_{50}$ values of $\mathrm{Nfs} A \_\mathrm{Li}$ with tinidazole was $178 \mu \mathrm{M}$, while the $\mathrm{IC}_{50} \mathrm{I}$ value for metronidazole was unable to be extrapolated, but from the graph we can see that it is $>400 \mu \mathrm{M}$.

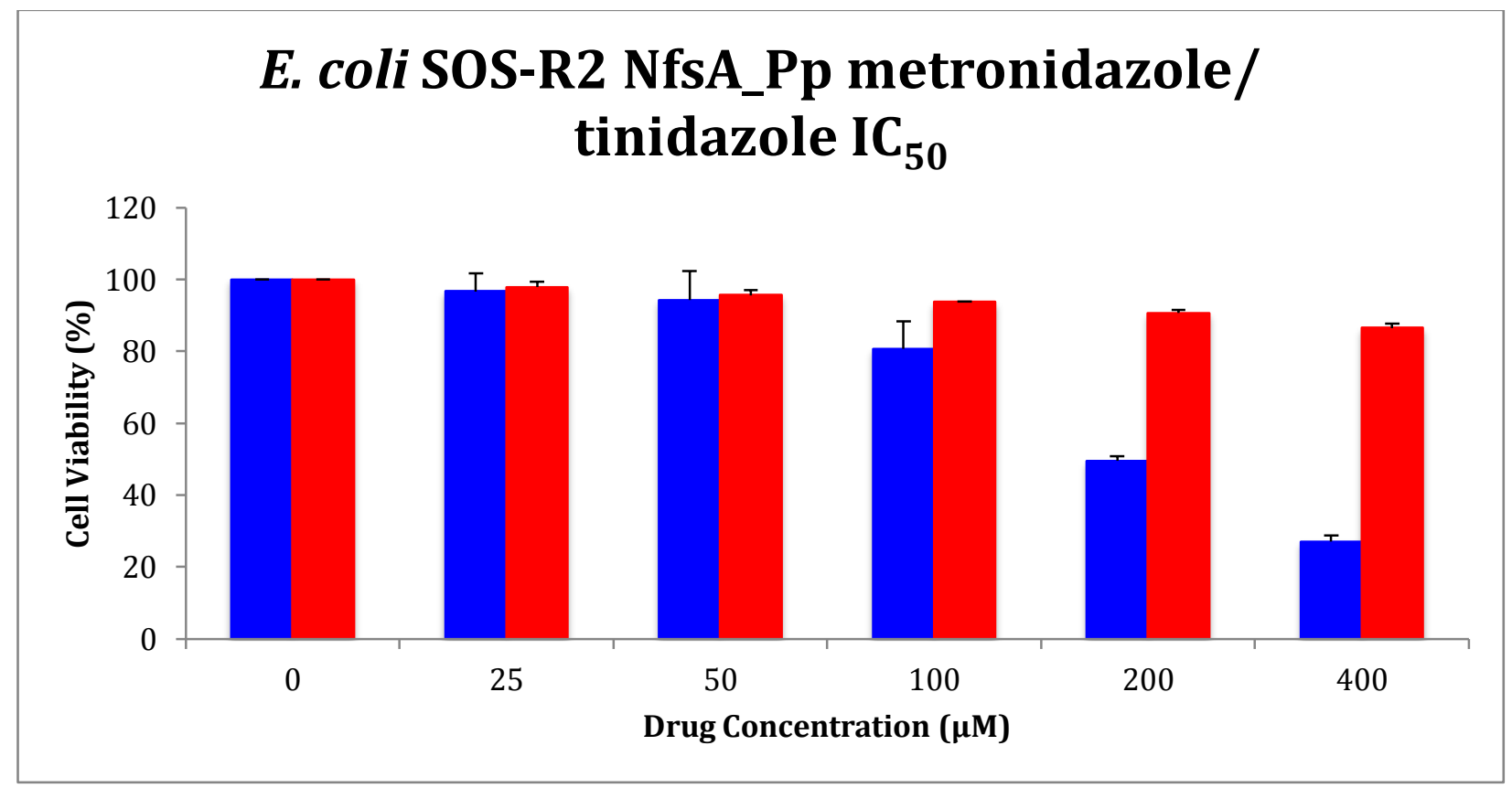

Figure 3.12 Metabolism of metronidazole and tinidazole by $\boldsymbol{E}$. coli cells over-expressing nitroreductase NfsA_Pp over a range of concentrations as measured by $\mathbf{I C}_{\mathbf{5 0}}$ analysis. Turbidity $\left(\mathrm{OD}_{600}\right)$ of nitroreductase over-expressing $E$. coli cultures was recorded directly before and after $4 \mathrm{~h}$ incubation with 5 different nil bystander antibiotic concentrations $(25 \mu \mathrm{M}, 50 \mu \mathrm{M}, 100 \mu \mathrm{M}, 200 \mu \mathrm{M}$ 
$400 \mu \mathrm{M})$. Metronidazole is shown in blue, and tinidazole red. Cell viability is expressed as the percentage decrease in $\mathrm{OD}_{600}$ of challenged cells relative to unchallenged control cells for each strain post-incubation. Data are the average of 3 independent assays and the error bars indicate \pm 1 standard deviation. The $\mathrm{IC}_{50}$ values of NfsA_Pp with tinidazole was $204 \mu \mathrm{M}$, while the $\mathrm{IC}_{50} \mathrm{I}$ value for metronidazole was unable to be extrapolated, but from the graph we can see that it is $>400 \mu \mathrm{M}$.

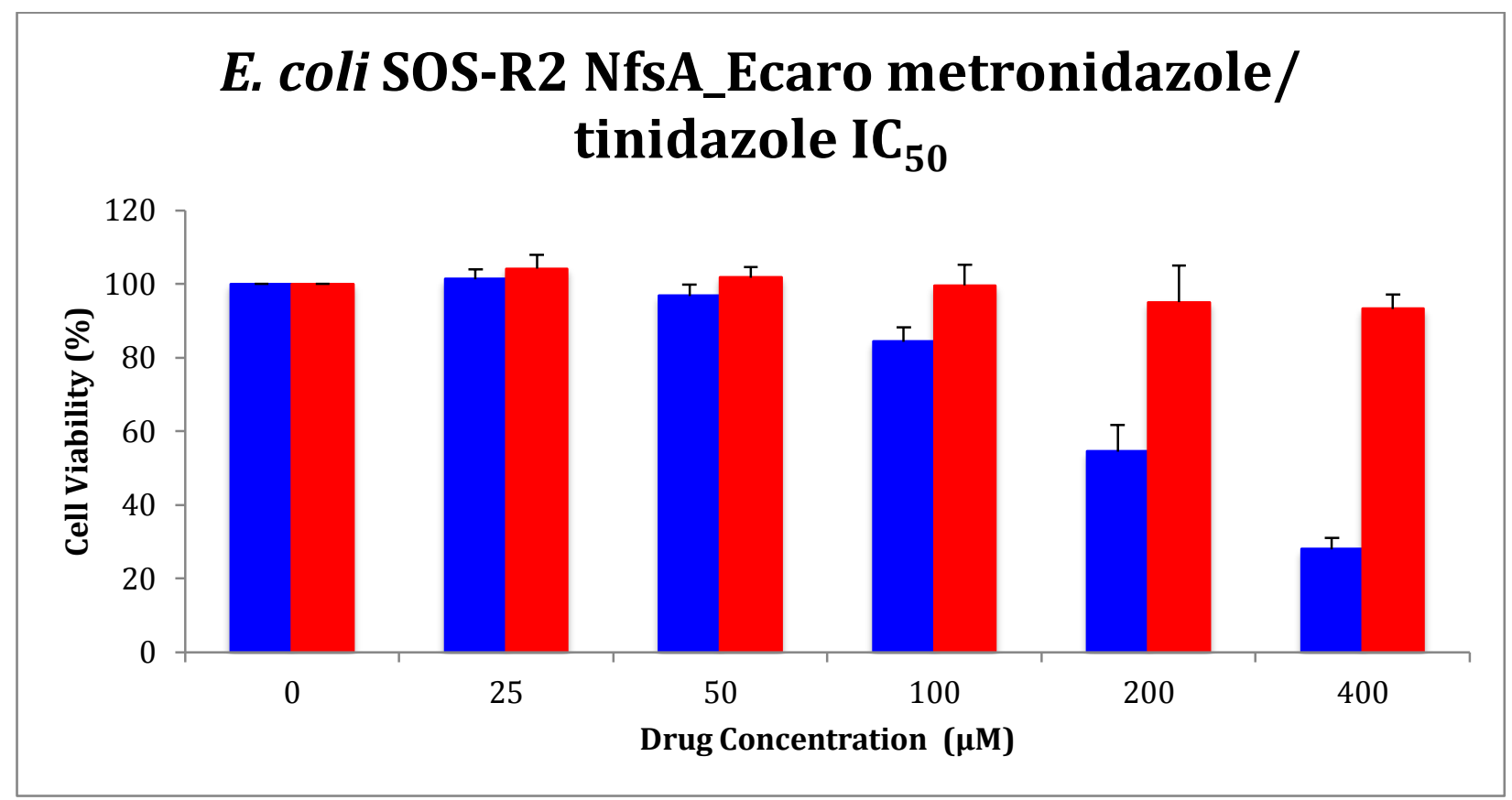

Figure 3.13 Metabolism of metronidazole and tinidazole by $E$. coli cells over-expressing nitroreductase NfsA_Ecaro over a range of concentrations as measured by $\mathrm{IC}_{\mathbf{5 0}}$ analysis. Turbidity $\left(\mathrm{OD}_{600}\right)$ of nitroreductase over-expressing $E$. coli cultures was recorded directly before and after $4 \mathrm{~h}$ incubation with 5 different nil bystander antibiotic concentrations $(25 \mu \mathrm{M}, 50 \mu \mathrm{M}, 100 \mu \mathrm{M}$, $200 \mu \mathrm{M} 400 \mu \mathrm{M})$. Metronidazole is shown in blue, and tinidazole red. Cell viability is expressed as the percentage decrease in $\mathrm{OD}_{600}$ of challenged cells relative to unchallenged control cells for each strain post-incubation. Data are the average of 3 independent assays and the error bars indicate \pm 1 standard deviation. The $\mathrm{IC}_{50}$ values of $\mathrm{NfsA}$ _Ecaro with tinidazole was $204 \mu \mathrm{M}$, while the $\mathrm{IC}_{50} \mathrm{I}$ value for metronidazole was unable to be extrapolated, but from the graph we can see that it is $>400 \mu \mathrm{M}$ 


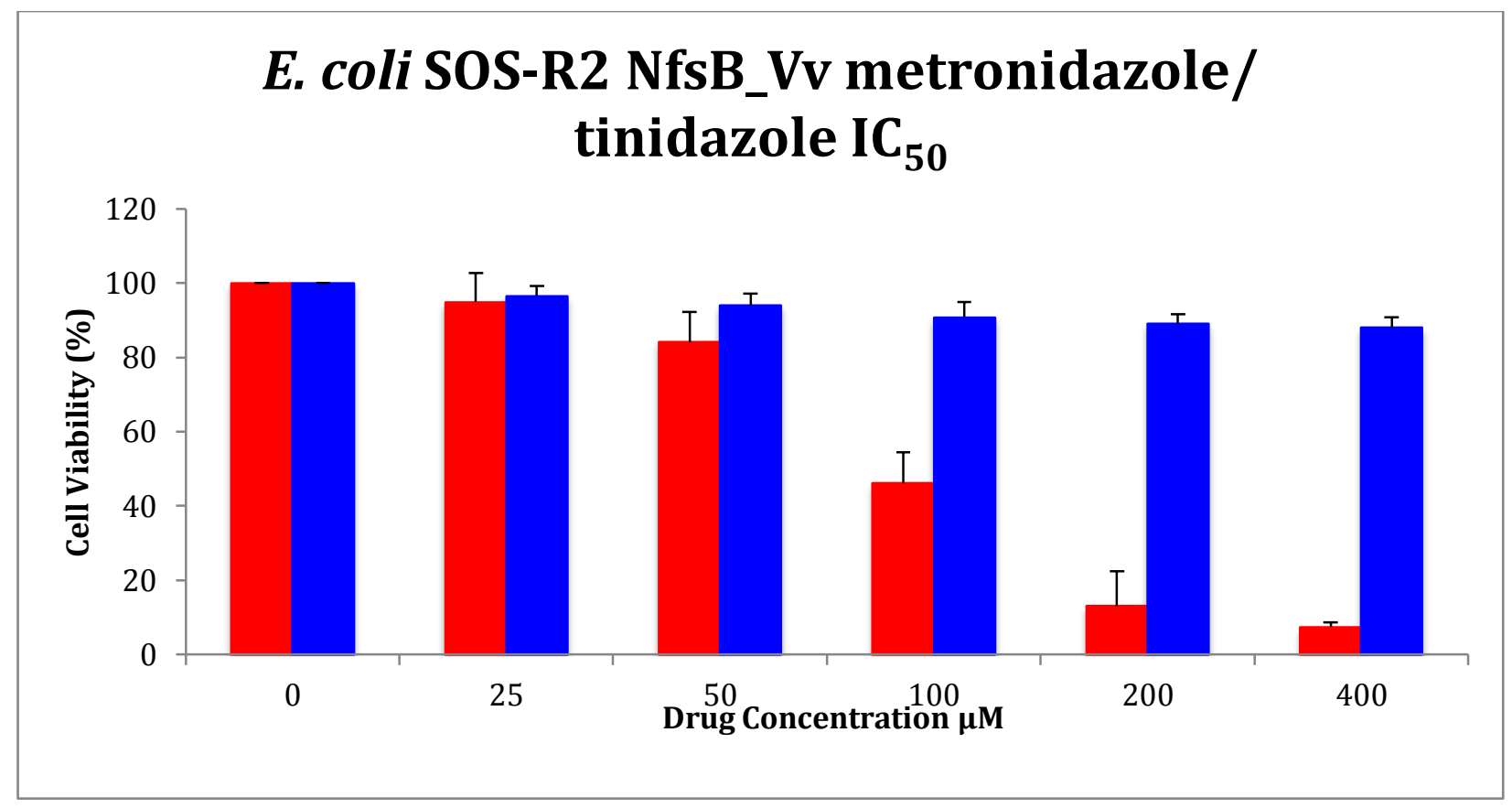

Figure 3.14 Metabolism of metronidazole and tinidazole by $E$. coli cells over-expressing nitroreductase $\mathbf{N f s B} \mathbf{V v}$ over a range of concentrations as measured by $\mathbf{I C}_{\mathbf{5 0}}$ analysis. Turbidity $\left(\mathrm{OD}_{600}\right)$ of nitroreductase over-expressing $E$. coli cultures was recorded directly before and after $4 \mathrm{~h}$ incubation with 5 different nil bystander antibiotic concentrations $(25 \mu \mathrm{M}, 50 \mu \mathrm{M}, 100 \mu \mathrm{M}, 200 \mu \mathrm{M}$ $400 \mu \mathrm{M})$. Metronidazole is shown in blue, and tinidazole red. Cell viability is expressed as the percentage decrease in $\mathrm{OD}_{600}$ of challenged cells relative to unchallenged control cells for each strain post-incubation. Data are the average of 3 independent assays and the error bars indicate \pm 1 standard deviation. The $\mathrm{IC}_{50}$ values of $\mathrm{NfsB}$ _Vv with metronidazole was $99 \mu \mathrm{M}$, while the $\mathrm{IC}_{50} \mathrm{I}$ value for tinidazole was unable to be extrapolated, but from the graph we can see that it is $>400 \mu \mathrm{M}$

The $\mathrm{IC}_{50}$ analysis showed the combination of nil bystander antibiotics metronidazole and tinidazole with the nitroreductases NfsA_Li and NfsB_Vv had the most potential to work as a dual ablation system. The ability of $\mathrm{NfsB}_{-} \mathrm{Vv}$ to be a strong activator of metronidazole had previously been looked at is it was initially one of the better candidates in place of NfsB_Ec, however NfsB_Vh showed a much better $\mathrm{IC}_{50}$ (table 3.1). Cell populations carrying the $\mathrm{NfsB}_{-} \mathrm{Vv}$ nitroreductase, were capable of activating metronidazole leading to cell death, while cells containing the NfsA_Li nitroreductase remained relatively unharmed. When cell populations were challenged with tinidazole, the opposite effect was observed; cell populations carrying the NfsA_Li nitroreductase were capable of activating tinidazole leading to cell death, while cells containing the $\mathrm{NfsB}$ _Vv nitroreductase remained relatively unharmed.. Although NfsA_Pp and NfsA_Ecaro also showed great potential to be used as tinidazole activating enzymes in partnership with metronidazole-activating NfsB_Vv, NfsA_Li was chosen as the best candidate as it had the least ability to activate metronidazole (Figure 3.7). It can also be seen clearly that metronidazole has the 
least growth inhibition effect on cells with NfsA_Li, as opposed to NfsA_Pp or NfsA_Ecaro from the $\mathrm{IC}_{50}$ analysis (Figures 3.11-3.14).

\subsubsection{Nitroreductase mediated cell ablation in human cell lines}

Previous work by others in the Ackerley lab, and at the Auckland Cancer Society Research Centre have shown that although the ability of nitroreductases to activate nil bystander antibiotics and severely inhibit growth in bacterial cells are a good indicator of what will be observed in mammalian cells, not all nitroreductases are able to be stably transfected into mammalian cells, meaning that not all results observed in bacterial cells will be able to be replicated in human cell lines

The nitroreductase with the highest efficacy with metronidazole - NfsB_Vh - had previously been transfected into HCT-116 cells by a past Ackerley lab member, Dr Horvat, so this was the first human cell line to be used for optimisation of experiments (Figure 3.4). NfsA_Ec and NfsB_Ec were subsequently transfected into human cell lines, as well as promising nitroreductase combinations that had previously shown opposing activation profiles in bacterial cells. This was carried out to test whether activation profile of these nitroreductases was able also applicable in human cell lines.

Dr Horvat previously encountered a great deal of difficulty in working with HCT-116 cell lines; As such, it was decided to work instead in the human embryonic kidney cell line HEK293, as these cells are commonly used as a generic working model in various experiments in human cell lines and are much more easily manipulated and transfected than HCT-116.

NfsA_Li and NfsB_Vv with tinidazole and metronidazole was the top nitroreductase/ nil bystander antibiotic combination identified above; these nitroreductases were therefore given the most preference to be transfected into HEK293. Time constraints and technical difficulties however hindered the generation of a stable HEK293 NfsA_Li cell line. However, a stable HEK293: NfsA_Pp cell line was successfully established and was used as an opposing activation profile nitroreductase - as it had a very similar activation profile to NfsA_Li (figures 3.11; 3.12). 


\subsubsection{Gateway $^{\mathrm{TM}}$ recombination system}

To transfer nitroreductase genes into HEK293 cells the Gateway recombination system developed by Invitrogen Life Technologies was employed (Hartley et al., 2000). The Gateway recombination system uses signal DNA sequence and enzymatic machinery of the $E$. coli bacteriophage lambda recombination system to achieve in vitro site-specific recombination (Hartley et al, 2000) at unique $7 \mathrm{bp}$ sequences denoted as att sites (attp in the entry clone and $a t t B$ on the target gene). Clonase ${ }^{\mathrm{TM}}$ enzyme mix mediates recombination occurring at these sites.

Two recombination reactions, LR and BP, constitute the Gateway cloning technology. The LR reaction is the in vitro version of the phage lambda excision reaction. Mediated by LR clonase through att $\mathrm{L}$ and att $\mathrm{R}$ sites, transfer of the target DNA fragment into a plasmid with to flanking recombination sequences attL entry clone, to a destination vector, a plasmid containing attR recombination sequences. These steps generate a clone with the attB sites flanking the gene of interest and capable of gene expression, known as an expression clone. The BP reaction transfers the DNA fragment of the Expression clone or the attB-flanked PCR product to a Donor vector through $a t t \mathrm{~B}$ and $\operatorname{att} \mathrm{P}$ by BP clonase, creating an entry clone (Sasaki et al., 2004).

For the transfection of nitroreductases into human cell lines, specific primer sets were designed to amplify chosen nitroreductases while incorporating Gateway recombination sites (table 2.7).

Recombination between $a t t B$-flanked PCR product of a nitroreductase gene and the vector donor pDONR221 (Invitrogen) to create an entry gene is the first step to generate nitroreductase Gateway clones. This is followed by a second recombination step where the entry clone is recombined with a destination vector to create an expression clone (figure 3.15).

Restriction digests carried out after these two recombination steps confirmed the insertion of nitroreductases into the vectors. The following human cell lines were successfully generated; HEK293: NfsA_Ec, HEK293: NfsB_Ec, HEK293: NfsA_Pp, 
A

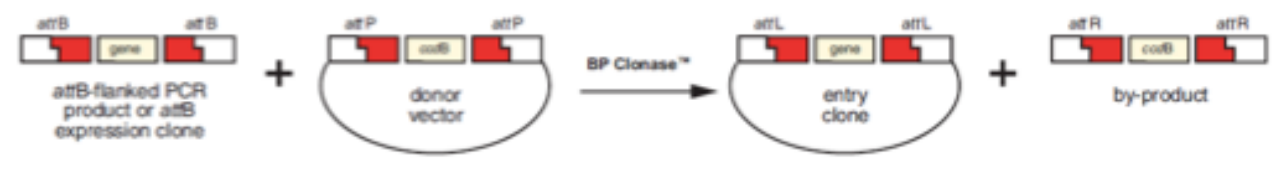

B

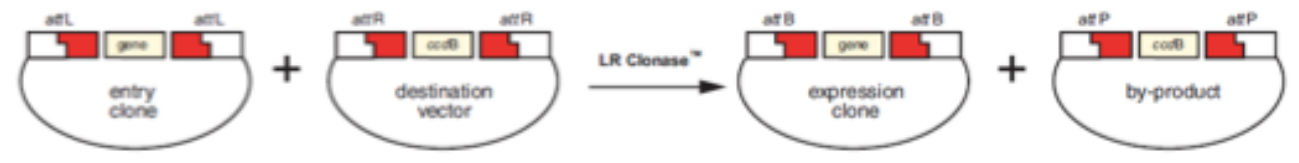

Figure 3.15 Overview of the Gateway ${ }^{\mathrm{TM}}$ recombination system. The recombination steps in creating expression vectors using the Gateway recombination cloning system. A) The BP recombination step, the PCR product of the gene of interest is recombined with the pDONR22 vector by recombination at the att sites to create an entry clone. B) The LR recombination step, the entry clone is recombined with a destination vector to create an expression strain that can then be transfected into the target cell line. Figure reproduced from the Invitrogen Gateway Technology Manual (www.invitrogen.com/content/sfs/manuals/gatewayman.pdf accessed 29.09.2013).

\subsubsection{Cell ablation in human cell lines}

The results observed in bacterial cells with NfsB_Vh, NfsA_Pp, and NfsB_Vv were predictive of what was observed in human cells. Initially MTS assay experiments were planned which would enable the $\mathrm{IC}_{50}$ values of given nil bystander antibiotics with the nitroreductases in human cell lines to be observed. However, problems with optimisation of the MTS assay meant that this work couldn't be verified in this manner. Instead another approach that would allow the visualisation of the ablation of the nitroreductase transfected cells in the presence of nil bystander antibiotics was undertaken. Cells were seeded into T25 and treated with $5 \mu \mathrm{M}$ drug - this drug concentration was based on previous MTS assays carried out by Dr Horvat, which indicated that after a $48 \mathrm{~h}$ period $5 \mu \mathrm{M}$ metronidazole was an optimal concentration to induce complete cell death in human cell lines. 


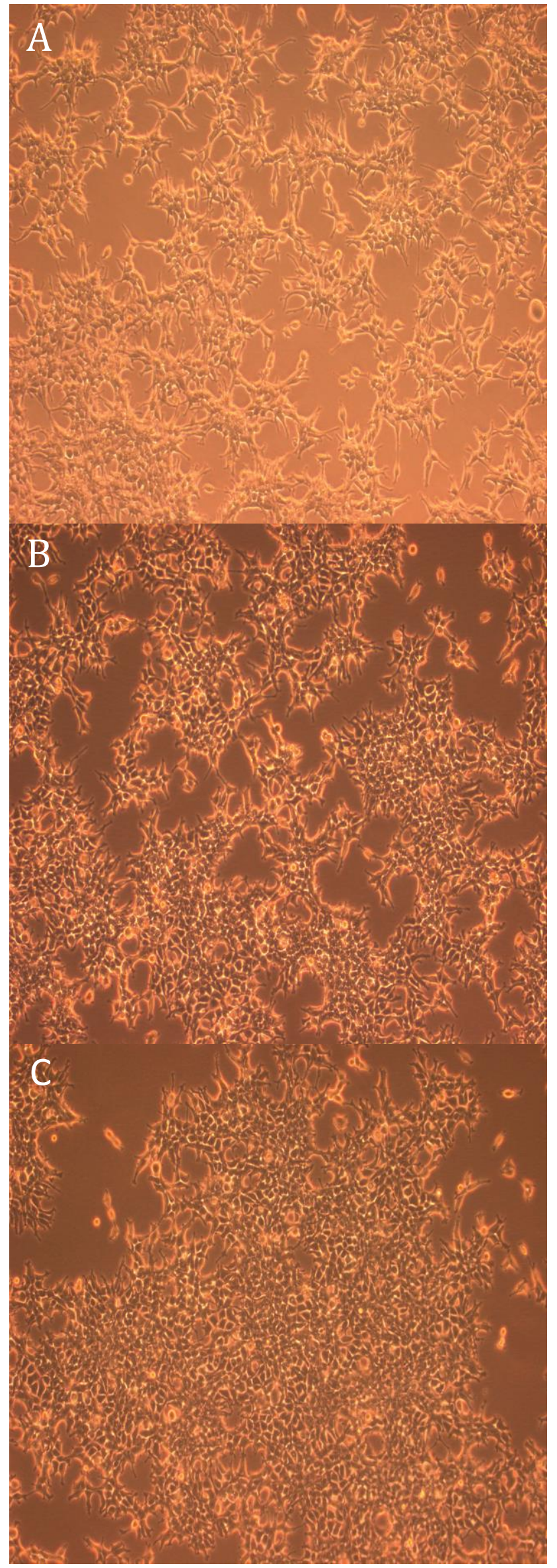

Figure 3.16 Metronidazole and tinidazole challenged HEK293 WT cells. Cells were seeded at a density of 250,000 into T25 flasks and left to grow overnight to approximately $80 \%$ confluency. $5 \mu \mathrm{M}$ metronidazole or $5 \mu \mathrm{M}$ tinidazole was added to the cells and they were left for $48 \mathrm{~h}$. Bright field images of the cell populations were taken at $0 \mathrm{~h}$ and $48 \mathrm{~h}$ with an Olympus IX51 microscope using the 20x lens. A. Unchallenged HEK293 WT cells B. HEK293 WT cells challenged with $5 \mu \mathrm{M}$ metronidazole $48 \mathrm{~h}$ C. HEK293 WT challenged with $5 \mu \mathrm{M}$ tinidazole $48 \mathrm{~h}$. After 48 hours in the presence of a low concentration of both metronidazole and tinidazole, the cells look healthy and the morphology appears unchanged from $0 \mathrm{~h}$. The cell population size appears to have increased over the 48 hours, suggesting that the presence of neither metronidazole nor tinidazole has any effect on the cells. 


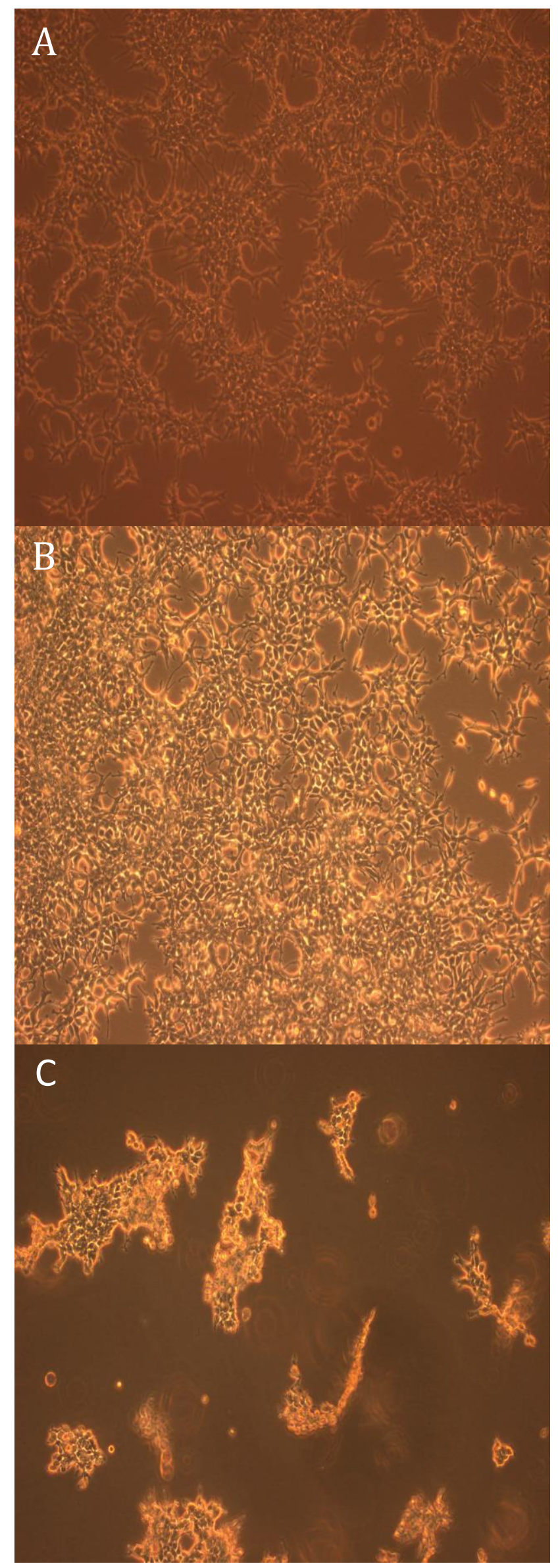

Figure 3.17 Metronidazole and tinidazole challenged HEK293: NfsA_Pp cells. Cells were seeded at a density of 250,000 into T25 flasks and left to grow overnight to approximately $80 \%$ confluency. $5 \mu \mathrm{M}$ metronidazole or $5 \mu \mathrm{M}$ tinidazole was added to the cells and they were left for $48 \mathrm{~h}$. Bright field images of the cell populations were taken at $0 \mathrm{~h}$ and $48 \mathrm{~h}$ with an Olympus IX51 microscope using the 20x lens. A.

Unchallenged HEK293 NfsA_Pp cells B. HEK293 NfsA_Pp cells challenged with $5 \mu \mathrm{M}$ metronidazole 48 h C. HEK293 NfsA Pp challenged with $5 \mu \mathrm{M}$ tinidazole 48 h. After 48 hours in the presence of a low concentration of tinidazole, the cells look unhealthy as the cell morphology has changed and population has decreased dramatically compared to when the population had not been in the presence of a nil bystander antibiotic. However, the addition of metronidazole appears to have no effect on HEK293: NfsA_Pp cell population, as the morphology appears unchanged from 0 $\mathrm{h}$, and the cell population size appears to have increased over the 48 hours. 


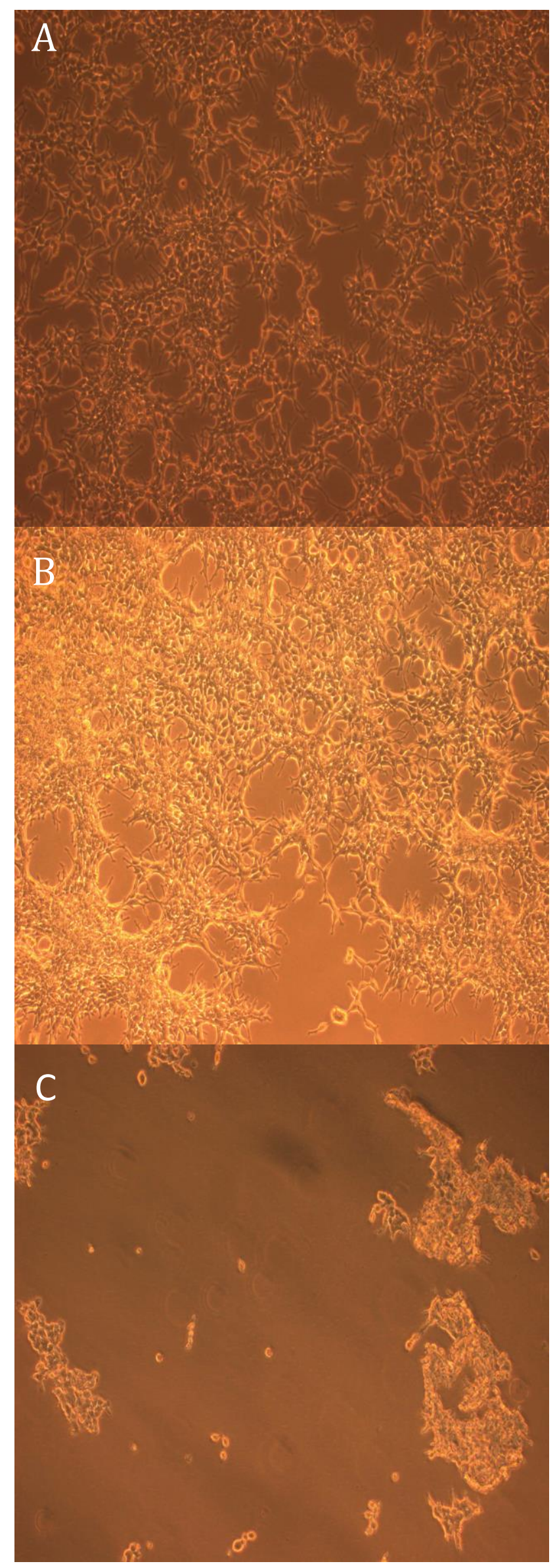

Figure 3.18 Metronidazole and tinidazole challenged HEK293: NfsB_Vv cells. Cells were seeded at a density of 250,000 into T25 flasks and left to grow overnight to approximately $80 \%$ confluency. $5 \mu \mathrm{M}$ metronidazole or $5 \mu \mathrm{M}$ tinidazole was added to the cells and they were left for $48 \mathrm{~h}$. Bright field images of the cell populations were taken at $0 \mathrm{~h}$ and $48 \mathrm{~h}$ with an Olympus IX51 microscope using the 20x lens. A. Unchallenged HEK293 NfsB_Vv cells B. HEK293 NfsB_Vv cells challenged with $5 \mu \mathrm{M}$ tinidazole $48 \mathrm{~h}$ C. HEK293 NfsA_Pp challenged with $5 \mu \mathrm{M}$ metronidazole $48 \mathrm{~h}$. After 48 hours in the presence of a low concentration of metronidazole, the cells look unhealthy as the cell morphology has changed and population has decreased dramatically compared to when the population had not been in the presence of a nil bystander antibiotic. However, the addition of tinidazole appears to have no effect on HEK293: NfsB_Vv cell population, as the morphology appears unchanged from 0 $\mathrm{h}$, and the cell population size appears to have increased over the 48 hours. 
The visualisation of stably transfected human cell lines treated with antibiotics was consistent with the results that had been seen in bacterial cells. HCT-116: NfsB_Vh showed high cell death after a $48 \mathrm{~h}$ incubation period with only $5 \mu \mathrm{M}$ metronidazole (figure 3.4), a much lower concentration than the $10 \mathrm{mM}$ that is currently being used in developmental and regenerative biology studies (Kaya et al., 2012). The cells analysed for their potential use in a dual ablation system also conferred the results we had expected based on earlier experiments in bacterial cells. HEK WT showed no cell death or change in cell morphology after a $48 \mathrm{~h}$ incubation period with either metronidazole or tinidazole (figure 3.16). HEK: NfsA_Pp showed high cell death after a $48 \mathrm{~h}$ incubation period with tinidazole, but no change in cell morphology after a $48 \mathrm{~h}$ incubation period with metronidazole (figure 3.17). HEK: NfsB_Vv showed high cell death after a $48 \mathrm{~h}$ incubation period with metronidazole, but no change in cell morphology after a $48 \mathrm{~h}$ incubation period with tinidazole (figure 3.18).

In summary, a number of promising pairs for use in a dual ablation system have been identified in bacterial cells. Proof of principle that this concept could be utilised in eukaryotic cells has been demonstrated using cell lines HEK293: NfsA_Pp and HEK293: NfsB_Vv in conjunction with the nil bystander antibiotics metronidazole and tinidazole. This offers great promise that these, or other, nitroreductases and nil bystander antibiotics could be implemented for use in eukaryotic model organisms as a means of targeted cell ablation in developmental biology studies.

\subsubsection{Niclosamide}

Niclosamide was one of the original nil bystander antibiotic candidates screened with the 58 nitroreductase library. During the initial screening process however, it was observed that while most nil bystander antibiotics had a similar activation pattern across the nitroreductases, niclosamide had the opposite activation profile, i.e. high levels of growth inhibition for the empty plasmid control, but low levels of growth inhibition for most strains expressing an active nitroreductase (Figure 3.6). The presence of active nitroreductases therefore appeared to be elucidating a protective effect on the cells, rather than a detrimental effect. This can be clearly illustrated by superimposing the activation profile of one of the other nil bystander antibiotics - in 
this case Metronidazole - onto a graph depicting niclosamide's activation profile

(Figure 3.19).

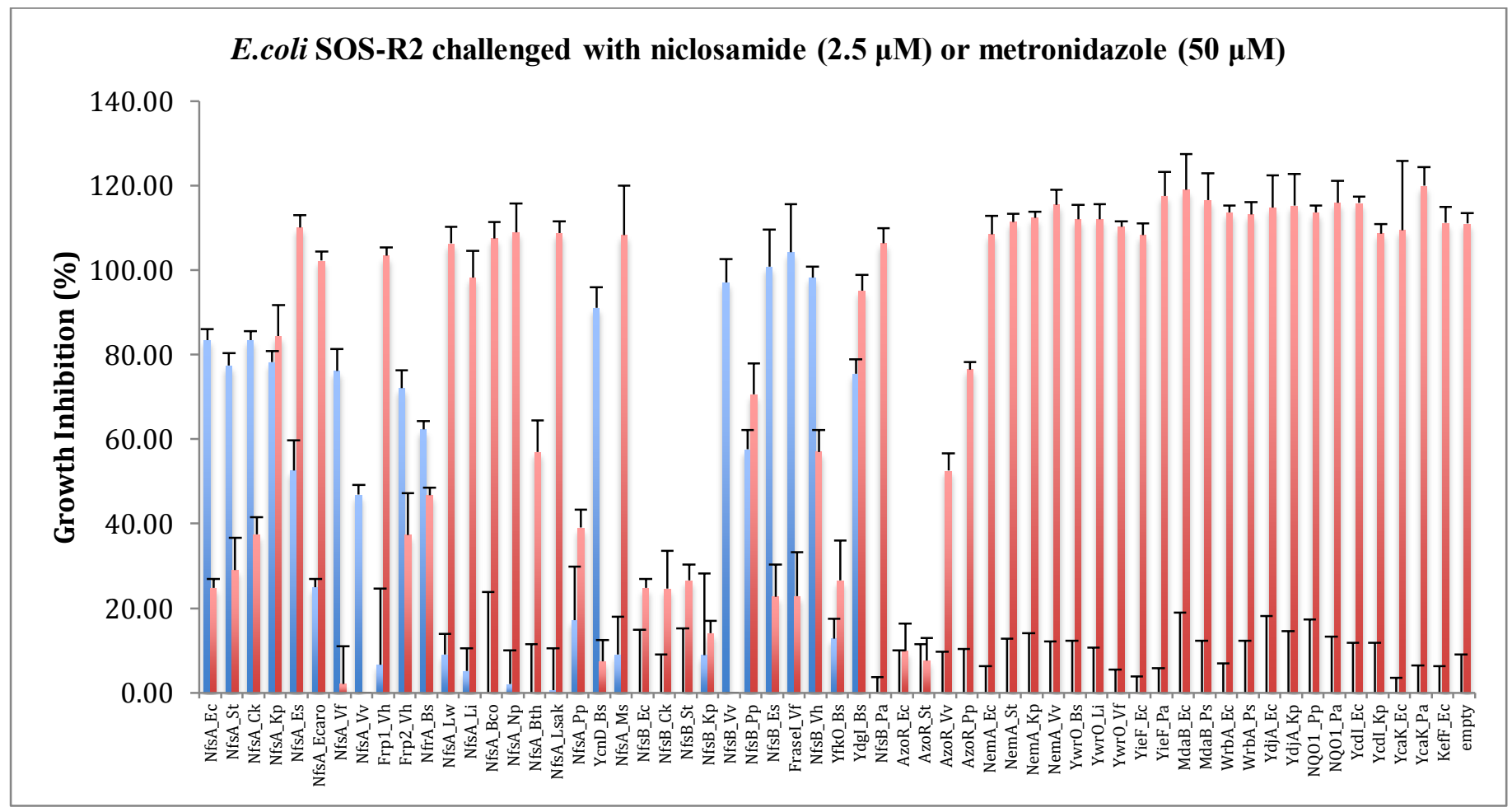

Figure 3.19 Metabolism of metronidazole or niclosamide by members of the 58 nitroreductase over-expression library as measured by growth inhibition assay. Turbidity $\left(\mathrm{OD}_{600}\right)$ of nitroreductase over-expressing $E$. coli cultures was recorded directly before and after $4 \mathrm{~h}$ incubation with $50 \mu \mathrm{M}$ metronidazole or $2.5 \mu \mathrm{M}$ niclosamide. Growth Inhibition is expressed as the percentage decrease in $\mathrm{OD}_{600}$ of challenged cells relative to unchallenged control cells for each strain post-incubation. Data are the average of 5 independent assays and the error bars indicate \pm 1 standard deviation. This graph shows the opposing activation profiles of metronidazole (blue) and niclosamide (red). Whereas with metronidazole, a lot of the NfsA and NfsB nitroreductase enzymes show high cell death, only cells expressing certain NfsA, NfsB or AzoR family members are able to grow in the presence of niclosamide, suggesting that these nitroreductases are exerting a protective effect on the cells as opposed to a detrimental effect.

\subsubsection{Exploiting the characteristics of niclosamide}

\subsubsection{Optimisation of niclosamide selection in liquid media}

Although niclosamide is not useful for the original intentions of this thesis, i.e. as a nitroreductase activated prodrug, its opposing activation profile to known nil bystander antibiotics suggests it might have value in a range of potentially useful applications. For example, niclosamide might have utility as a high throughput positive selection agent to identify novel nitroreductases in large genetic (e.g. 
metagenomic, or directed evolution) libraries. However, prior to any such applications it was necessary to better understand the concentrations of niclosamide that should be applied for effective selection.

Although not ultimately pursued, it was first sought to examine the effects of niclosamide challenge in conjunction with fluorescence screening. This was to be tested using nitro-quenched fluorophores, molecules that will be discussed in greater detail in Chapter 4. For now, the critical detail is that in order to apply these fluorophores it was necessary to shift from the E. coli SOS-R2 strain in which all growth inhibition assays described above had been conducted, to a different strain, $E$. coli $6 \mathrm{KO}$ (whereas SOS-R2 has had four endogenous nitroreductase genes knocked out, the 6KO strain has six nitroreductase gene deletions (Table 2.3); this has been found to be important for minimising background in fluorescence assays; Horvat, 2012). However, it quickly became apparent that the $6 \mathrm{KO}$ strain responded quite differently to niclosamide challenge than SOS-R2. Numerous experiments were conducted using the full NTR library in both strains. However, for simplicity, the remainder of this section will just describe key observations made for NfsA_Vv expressing "nitroreductase positive" cells versus the pUCX empty plasmid negative control cells.

Initial experiments aiming to optimise niclosamide challenge concentrations were undertaken in liquid media as described in Section 2.9.7. The $\mathrm{OD}_{600}$ readings for both NfsA_Vv and pUCX were taken at four different time points, $0 \mathrm{~h}, 2 \mathrm{~h}, 4 \mathrm{~h}$, and $8 \mathrm{~h}$, across a range of niclosamide concentrations. The results that were observed in $6 \mathrm{KO}$ however, were not consistent with those observed in the SOS-R2 strain. Whereas the NfsA_Vv over-expressing $6 \mathrm{KO}$ strain behaved as expected, showing increased $\mathrm{OD}_{600}$ readings at later time points (consistent with a protective role for the over-expressed nitroreductase), the empty plasmid control also showed increased $\mathrm{OD}_{600}$ readings across all time points, indicating that niclosamide was not inhibiting its growth (Figure 3.21). The same experiments were then carried out using another nitroreductase gene-deleted strain available in the lab, $7 \mathrm{KO}$, with the same results (Figure 3.22). In contrast, when the experiment was repeated in SOS-R2, the empty plasmid control strain was once again growth-inhibited by niclosamide (Figure 3.23). 


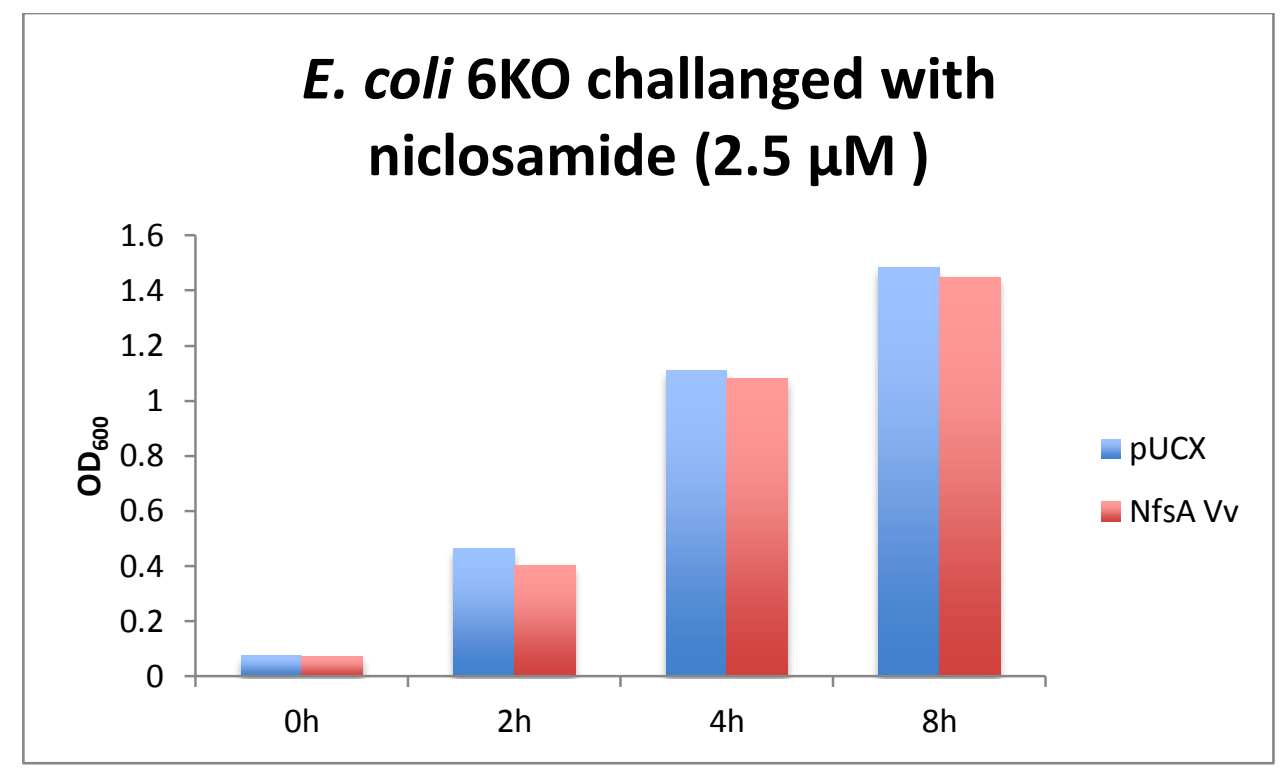

Figure 3.20 Metabolism of niclosamide by E.coli 6KO overexpressing NfsA_Vv or pUCX over an $8 \mathrm{~h}$ time period. The $E$. coli $6 \mathrm{KO}$ strain containing either NfsA_Vv as a positive control or pUCX as a negative control was challenged with $2.5 \mu \mathrm{M}$ niclosamide. $\mathrm{OD}_{600}$ values were taken at four different time points $0 \mathrm{~h}, 2 \mathrm{~h}, 4 \mathrm{~h}$, and $8 \mathrm{~h}$. Results observed were inconsistent with those seen in growth inhibition assays when the $E$. coli SOS-R2 58 nitroreductase library was challenged with $2.5 \mu \mathrm{M}$ niclosamide for $4 \mathrm{~h}$. It was expected that there would be growth inhibition of cells containing pUCX, but not cells expressing NfsA_Vv. In the E. coli $6 \mathrm{KO}$ strain no growth inhibition with either NfsA_Vv or pUCX was observed.

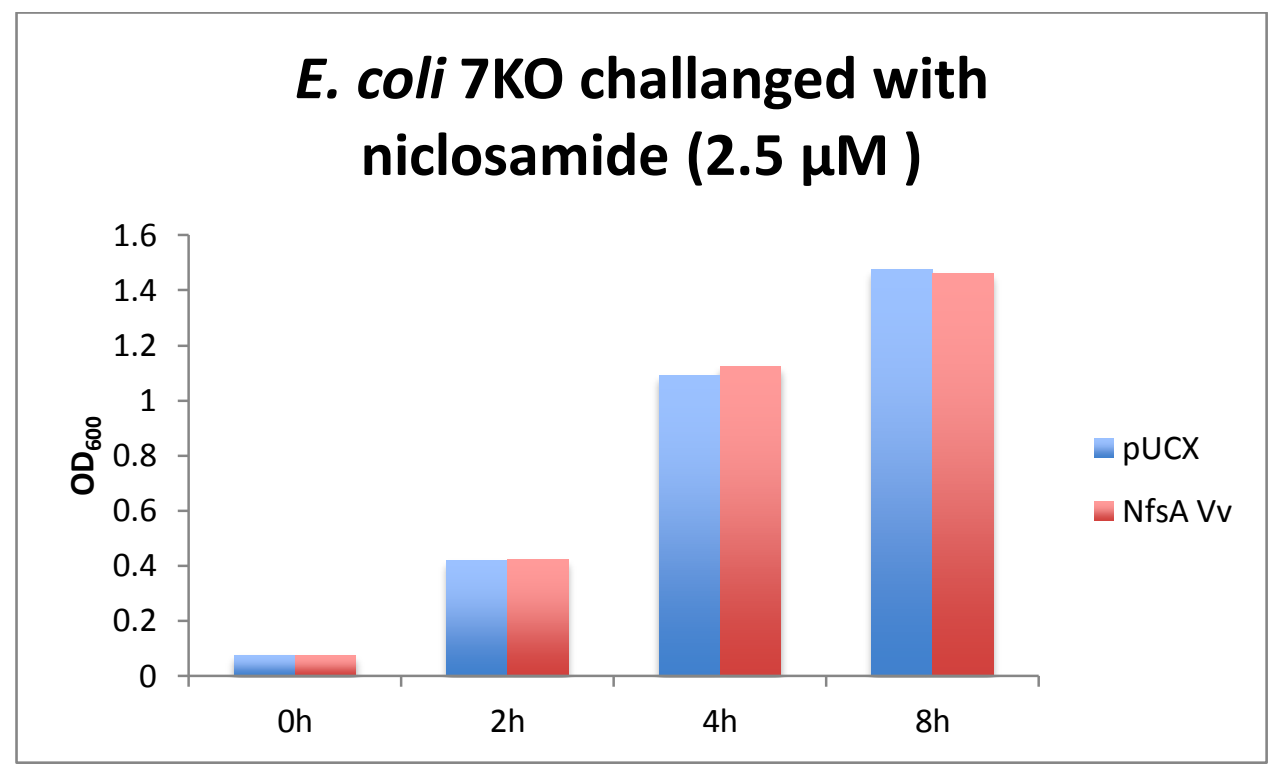

Figure 2.21 Metabolism of niclosamide by E.coli 7KO overexpressing NfsA_Vv or pUCX over an $8 \mathrm{~h}$ time period. The $E$. coli $7 \mathrm{KO}$ strain containing either NfsA_Vv as a positive control or pUCX as a negative control was challenged with $2.5 \mu \mathrm{M}$ niclosamide. $\mathrm{OD}_{600}$ values were taken at four different time points $0 \mathrm{~h}, 2 \mathrm{~h}, 4 \mathrm{~h}$, and $8 \mathrm{~h}$. Results observed were inconsistent with those seen in growth inhibition assays when the $E$. coli SOS-R2 58 nitroreductase library was challenged with $2.5 \mu \mathrm{M}$ niclosamide for $4 \mathrm{~h}$. It was expected that there would be growth inhibition of cells containing pUCX, but not cells expressing NfsA_Vv. In the E. coli $7 \mathrm{KO}$ strain no growth inhibition with either NfsA_Vv or pUCX was observed. 


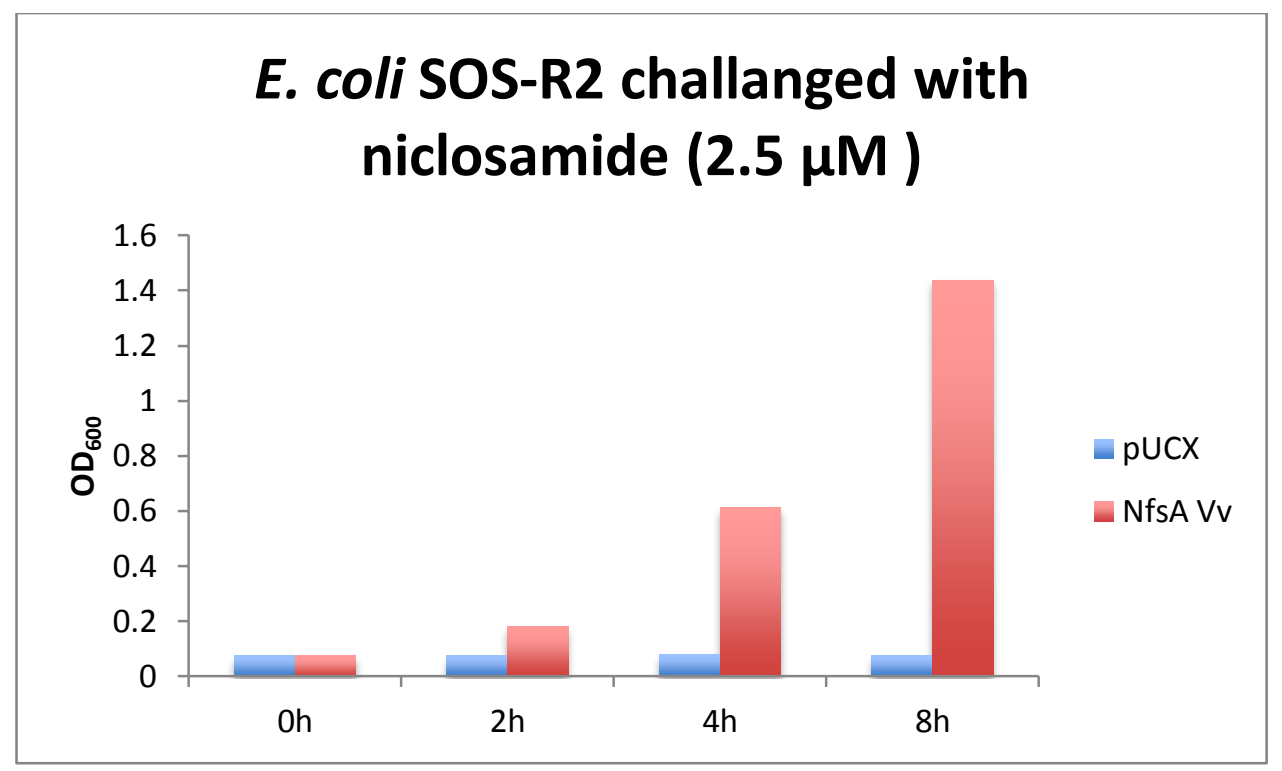

Figure 3.22 Metabolism of niclosamide by E.coli SOS-R2 overexpressing NfsA_Vv or pUCX over an $8 \mathrm{~h}$ time period. The $E$. coli SOS-R2 strain containing either NfsA_Vv as a positive control or pUCX as a negative control was challenged with $2.5 \mu \mathrm{M}$ niclosamide. $\mathrm{OD}_{600}$ values were taken at four different time points $0 \mathrm{~h}, 2 \mathrm{~h}, 4 \mathrm{~h}$, and $8 \mathrm{~h}$. Results observed agree with those which had been seen in growth inhibition assays when the E. coli SOS-R2 58 nitroreductase library was challenged with 2.5 $\mu \mathrm{M}$ niclosamide for $4 \mathrm{~h}$. Growth inhibition was observed in cells containing pUCX, but not in cells containing NfsA_Vv.

Apart from containing fewer nitroreductase gene knockouts, the only overt difference between SOS-R2 and the $6 \mathrm{KO} / 7 \mathrm{KO}$ strains is that SOS-R2 also lacks a gene encoding the TolC efflux regulator. It was therefore inferred that TolC might be playing an active role in minimising the toxic effect of niclosamide on $E$. coli cells.

The role of the TolC efflux regulator is to expel a number of diverse molecules, including protein toxins and antibiotic drugs, from the cell (Thanabalu et al, 1998, Koronakis et al 2004). We hypothesised that TolC is able to cause niclosamide to be rapidly eliminated from $E$. coli cells, rendering nitroreductase-mediated protection redundant. Therefore, to utilise this anomaly observed with niclosamide in combination with a masked fluorophore screening method to confirm the presence of active nitroreductases, another member of the lab, Dr Janine Copp, developed a new strain with a tolC gene knockout; 7NT. 7NT otherwise has the same characteristics as $7 \mathrm{KO}$ (table 2.3). As previously observed in SOS-R2 cells, in the 7NT strain $2.5 \mu \mathrm{M}$ niclosamide was found to be an effective concentration for preventing the growth of the pUCX control strain, while allowing the NfsA_Vv expressing strain to grow freely (not shown). 


\subsubsection{Optimisation of niclosamide selection in solid media}

The Ackerley lab has an interest in discovering new nitroreductase enzymes from metagenome libraries. These libraries provide access to the genetic material of the $>99 \%$ of microbes that cannot be cultured in the lab (Vartoukian et al., 2010). Functional screens of such libraries are a very powerful tool to recover enzymes from these libraries (van Rossum et al., 2013). The observation made here, that a tolC and endogenous nitroreductase gene deleted strain of $E$. coli will be unable to grow in the presence of niclosamide unless an alternative (protective) nitroreductase gene is supplied, suggested a means for screening metagenome libraries to recover clones encoding novel nitroreductases. While working in liquid media provided some essential insights into the effect of activated niclosamide on various $E$. coli strains, it is not ideal for this kind of screening as working in liquid media may lead to a minority of nitroreductase expressing cells completely detoxifying niclosamide and thereby allowing the vast majority of non-resistant clones to proliferate. To utilise the characteristics observed in niclosamide in this context, it was preferable to demonstrate activity on solid media.

Another purpose of the niclosamide validation experiments was to establish whether the system could also be used in the future for directed evolution, selecting for loss of specific nitroreductase activities. This would be useful in gaining further combinations of nitroreductases/ nil bystander antibiotics with opposing activation profiles, or for tailoring the activity of nitroreductases already identified as showing promise in an opposing activation profile context. For example, NfsA_Pp wasn't the first choice of nitroreductase to be used in the dual ablation system outlined in 3.2.3, and was only selected because NfsA_Li was unable to be expressed functionally in human cell lines. Whereas both NfsA_Li and NfsA_Pp are highly active with tinidazole, unlike NfsA_Li, NfsA_Pp shows low-level activation of metronidazole. The ability to eliminate any possibility of NfsA_Pp activating metronidazole would make the proposed dual ablation system (in combination with NfsB_Vv) much more targeted. One strategy proposed to achieve this was to randomly mutate the gene encoding NfsA_Pp, to express the variant gene library in SOS-R2 or 7NT cells, and then to plate that library on media containing both a high level of metronidazole and niclosamide. Only variants that have lost activity with metronidazole should be able 
to grow; whereas the inclusion of niclosamide in the growth medium would ensure that at least some level of generic nitroreductase activity is retained. Surviving variants could then be tested to ensure an appropriate level of activity with tinidazole has been retained.

To test the feasibility of this idea, initial experiments were undertaken in the SOS-R2 strain with controls first tested using plates containing metronidazole only, or niclosamide only. Colonies that were able to grow in the presence of niclosamide indicate the presence of a nitroreductase, while the ability to also grow in the presence of metronidazole indicates that the nitroreductase cannot activate metronidazole. Again, as in the liquid media optimisation experiments, NfsA_Vv was used as a positive control, as it is a well-validated nitroreductase but has only poor activity with metronidazole. Alongside NfsA_Vv, pUCX was employed as a negative control. When NfsA_Vv was plated on solid media containing mertronidazole or niclosamide only, colonies formed, whereas pUCX containing colonies only formed on plates containing metronidazole. These outcomes established the parameters for niclosamide selection of active nitroreductases from mixed cultures.

7NT strain cells expressing either NfsA_Vv or pUCX were then grown in separate cultures, before being mixed at a 1:1 ratio and plated on solid media containing 10 $\mu \mathrm{M}$ metronidazole and $2.5 \mu \mathrm{M}$ niclosamide. To confirm the growth of only E. coli colonies containing an active nitroreductase (NfsA_Vv) PCR analysis of randomly picked colonies this plate was undertaken. The PCR colony screen confirmed that all colonies growing contained the nitroreductase insert NfsA_Vv; none contained empty pUCX (figure 3.23). 


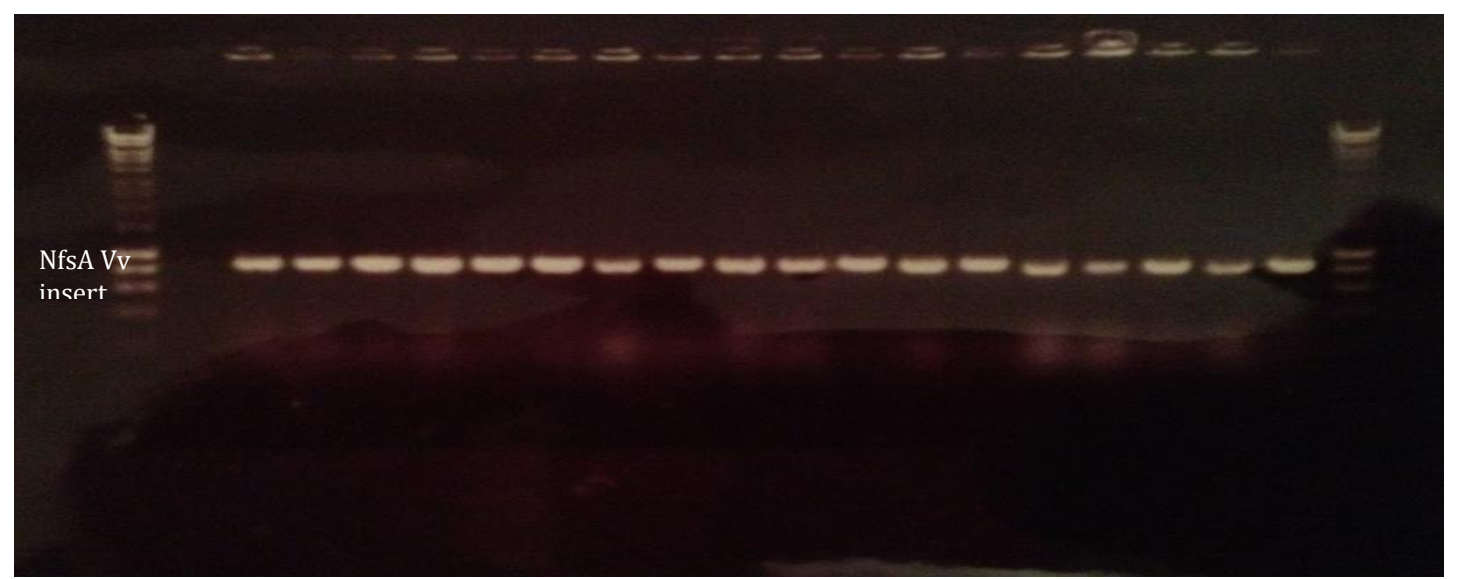

Figure 3.23 PCR confirmation of active nitroreductase enzymes from colonies picked from mixed culture NfsA_Vv/ pUCX plated on media containing metronidazole and niclosamide. 17 colonies were picked from plates containing both metronidazole and niclosamide.. The colonies were then amplified by PCR using generic nitroreductase specific primers. A negative control, pUCX picked from a no drug pUCX only plate, (lane 1) and a positive control, NfsA_Vv picked from a no drug NfsA_Vv only plate, (lane 2) were also run. All randomly picked colonies from plates containing both niclosamide and metronidazole show the NfsA_Vv insert.

Colonies were also picked and challenged with masked fluorophore FSL41. The colonies containing the nitroreductases were able to activate FSL41 and give off a fluorescence signal, while those not containing the nitroreductase wouldn't. All of the colonies that were picked showed fluorescence in comparison with the pUCX negative control. Fluorescence was visualised on the UV Trans-illuminator, and measured on the Enspire 2300 Multiplate Reader at ex $355 \mathrm{~nm} / \mathrm{em} 460 \mathrm{~nm}$.

Although the image taken on the UV trans-illuminator is slightly over-exposed (figure 3.24), the emission of blue fluorescence can clearly be seen from the raw fluorescence units as detected on the plate reader (figure 3.25)

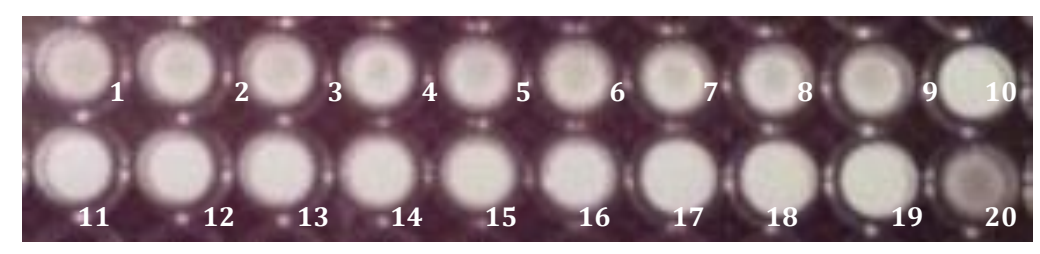

Figure 3.24 FSL41 fluorescence visualised on UV trans-illuminator as confirmation of active nitroreductase enzymes from colonies picked from mixed culture NfsA_Vv/ pUCX plated on media containing metronidazole and niclosamide. 18 random colonies were picked from plates containing both metronidazole and niclosamide (wells 1-18). A negative control, pUCX picked from a no drug pUCX only plate, (well 20) and a positive control, NfsA_Vv picked from a no drug NfsA_Vv only plate, (well 19) were also treated with FSL41. Each colony was incubated in LB overnight, then followed the standard fluorescence screening assay (2.9.4) the following day. FSL41 was added to each well to a final concentration of $25 \mu \mathrm{M}$ and incubated for $4 \mathrm{~h}$. Wells were then visualised under the UV trans-illuminator, with wells containing NfsA_Vv activating FSL41 allowing the emission of blue fluorescence. The randomly picked colonies and the NfsA_Vv positive control are emitting fluorescence, while the pUCX negative control is not, confirming that all randomly picked colonies contain NfsA_Vv. 


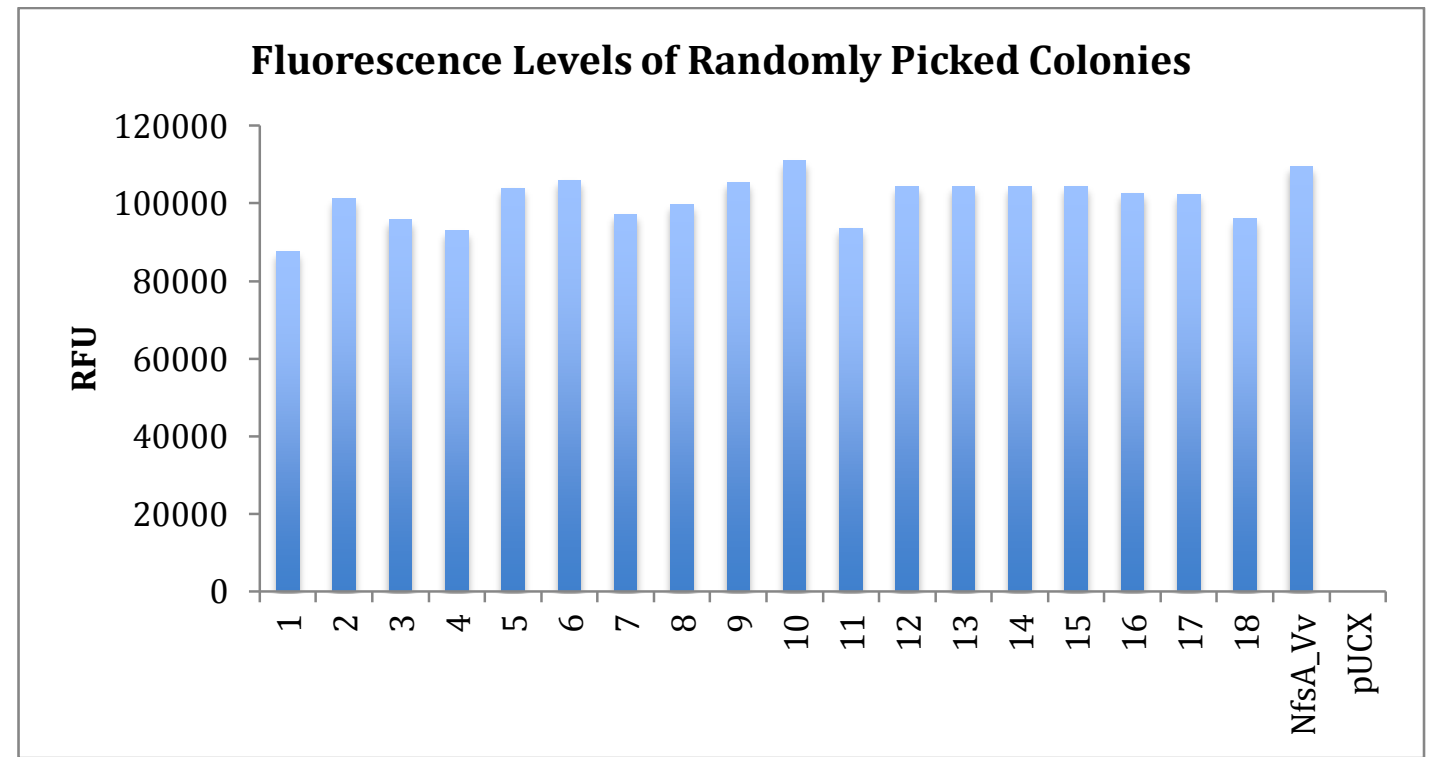

Figure 3.25 FSL41 fluorescence plate reader ex $355 \mathrm{~nm} / \mathrm{em} 460 \mathrm{~nm}$ values as confirmation of active nitroreductase enzymes from colonies picked from mixed culture NfsA_Vv/ pUCX plated on media containing metronidazole and niclosamide. 18 random colonies were picked from plates containing both metronidazole and niclosamide (1-18). A negative control, pUCX picked from a no drug pUCX only plate, and a positive control, NfsA_Vv picked from a no drug NfsA_Vv only plate, were also treated with FSL41. Each colony was incubated in LB overnight, then followed the standard fluorescence screening assay (2.9.4) the following day. FSL41 was added to each well to a final concentration of $25 \mu \mathrm{M}$ and incubated for $4 \mathrm{~h}$. The plate was then read on the Enspire 2300 Multiplate Reader. Wells containing NfsA_Vv activate FSL41 allowing the emission of blue fluorescence, the background fluorescence levels from the negative pUCX control were subtracted from the final RFU of each well. All of the randomly picked colonies are emitting fluorescence, as the RFU are all around the same value as the NfsA_Vv positive control.

\subsection{Discussion}

The screening of the 58 nitroreductase core library with the 16 nil bystander antibiotic candidates was effective in identifying which nitroreductases were capable of reducing a given nil bystander antibiotic, suggesting their possible utility as a safety mechanism in GDEPT; an FDA requirement for this technology being the ability to eliminate biological vectors from patients post-therapy or if PET imaging indicates undesirable vector localisation as pathogenic bacterium, such as Clostridium sporogenes, are commonly used vectors in solid tumour targeting. All but 4 of the putative nil bystander antibiotics (dimetridazole, EF5, F-misonidazole, ronidazole) screened against the 58 nitroredutase library have previously been approved for use in the clinic. Our collaborator at the Auckland Cancer Society Research Centre, Dr Adam Patterson, is currently testing several nil bystander antibiotic candidates for the ability to eliminate both Clostridium and adenovirus nitroreductase-labelled vectors 
from human cell cultures. Clearly, it would be advantageous for any nil bystander antibiotics going forward for use in a GDEPT vector ablation system to have already made it into the clinic. From the heat-map (Figure 3.6) we can see that $N$ Benznidazole $(40 \mu \mathrm{M})$ exhibited $100 \%$ growth inhibition with the majority of the NfsA family, and might therefore constitute an especially promising candidate for this role. However, it would first be necessary to confirm $\mathrm{N}$-Benznidazole is truly a nilbystander prodrug; this could be measured in mixed multicell layers as described by Wilson et al., (2002, 2003).

Of the nitroreductases that were identified as being superior at activating metronidazole to NfsB_Ec, NfsB_Vh had the highest growth inhibition percentage (figure 3.2), and these results were confirmed by running $\mathrm{IC}_{50} \mathrm{~S}$ alongside other potential nitroreductases with higher efficacy as well as NfsB_Ec itself (Figure 3.3). Once this had been established, the results were translated into human cells, as the technology in which this nitroreductase would be used is in eukaryotic cells. Initial results showed that the morphology of the HCT-116 cells with the NfsB_Vh nitroreductase was compromised in the presence of metronidazole. However, challenging HCT-116: NfsB_Vh with metronidazole at lower metronidazole concentrations, which did not compromise the morphology of the human cell line, confirmed that NfsB_Vh was capable of efficiently ablating HCT-116 population at very low concentrations (figure 3.4). The identification of this nitroreductase with a much higher ability to activate metronidazole compared to NfsB_Ec, and its ability to translate this effect into eukaryotic cells, could be of huge potential for the study of the role of specific cell types during development. Further testing will be required to validate this potential in developmental cell biology models, such as transgenic zebrafish lines.

The majority of studies exploiting the nitro reducing capabilities of these enzymes focus on the $E$. coli nitroreductases $\mathrm{NfsA}$ and $\mathrm{NfsB}$, as these have the most literature available and are the most easily accessible. A useful resource has been the access to a 58 nitroreductase library that has been developed in the Ackerley laboratory as it is unique in the number and variety of nitroreductases available. 
The screening of the whole 58 nitroreductase core library by growth inhibition assay enabled the identification of which nil bystander antibiotics are highly active with any individual nitroreductase. The data obtained from these early screening assays were collated into an easy to interpret colour coded heat-map (Figure 3.6). This made it simple to identify of pairs nil bystander antibiotics/ nitroreductase combinations that have opposing activation profiles. This is something that that could potentially be used in a dual targeted cell ablation system. For example, it has already been demonstrated that the beta cells of the pancreas can be specifically ablated via metronidazole treatment of transgenic zebrafish expressing NfsB_Ec under control of an insulin promoter. This could potentially be combined with the targeting of alpha cells of the pancreas to compare and contrast the effects to the organism of knocking out these different cell types during development. Such a multiplex capability could greatly extend the power of nitroreductases as useful cell biology tools

$\mathrm{IC}_{50}$ analysis of pairs of nil bystander antibiotics with opposing activation profiles also confirmed the results that had been initially observed in the growth inhibition assays. The pair that was initially given the most attention was the NfsA_Li/ tinidazole and NfsB_Vv/ metronidazole combination. This combination was desirable as it is an NfsA family/ NfsB family comparison (advantages of such a pairing include the existence of fluorogenic probes that can accurately discriminate the two families, as will be seen in the next chapter). Moreover, the nil bystander antibiotics that each enzyme was active with are very well studied, especially metronidazole; which is already being used in studies looking at single cell ablation in various organisms (Curado et al., 2007 Pisharath et al,. 2009, White et al., 2011).

All initial tests were performed in bacterial cells, as this allowed flexible and high throughput evaluation of nitroreductase potential. However, the intended applications of these enzyme/prodrug systems are in eukaryotic cells. It was therefore important to demonstrate that the activation profiles that had been seen in bacterial strains were readily transferable to eukaryotic cell lines. Most of the nitroreductases were transfected into HEK293 as this human cell line is well-known and easy to work in. Unfortunately due to time constraints and technical difficulties, NfsA_Li was unable to be transfected into HEK293. It is not known if NfsA_Li is able to be stably transfected into human cell lines, as it has been previously observed that not all 
nitroreductases are able to be transfected into human cell lines with stable expression (Prosser, 2013). Instead, a HEK293: NfsA_Pp cell line was established; this nitroreductase exhibits a similar activation profile to NfsA_Li (i.e. high activity with tinidazole and low activity with metronidazole); and the transfected cell line was used as an opposing activation profile to partner with HEK293: NfsB_Vv.

As was the case with HCT-116: NfsB_Vh treated with metronidazole (Figure 3.4), the morphology of HEK293: NfsA_Pp and HEK293 NfsB_Vv were compromised in the presence of tinidazole and metronidazole respectively (Figures 3.17; 3.18). Looking at opposing activation profiles however had an added layer of complexity: for the technology to be feasible, HEK29: NfsA_Pp had to not be affected by metronidazole, and HEK293: NfsB_Vv resistant to tinidazole. Microscopy images of this (Figures $3.16 ; 3.17)$ showed that there was little effect on the cells when in the presence of the opposing nil bystander antibiotic. As a negative control HEK293 WT cells were also challenged with both metronidazole and tinidazole and visualised after a $48 \mathrm{hr}$ time period. Neither nil bystander antibiotic had any visible effect on the morphology of HEK293 WT (figure 3.16) confirming that the nitroreductase present was responsible for the activation of the nil bystander antibiotic and subsequent cell ablation.

All of these results were consistent with the drug activation profiles in bacterial cells and are a promising proof of principle for future dual ablation work, as well as the possibility of using this in developmental and regenerative biology studies as a novel approach to targeted cell ablation.

A different aspect of this study was that while undertaking the initial screening of the 58 nitroreductase core library against the 16 nil bystander antibiotic candidates, an anomaly was observed when niclosamide consistently showed an activation profile that was consistently the opposite of that observed with other nil bystander antibiotics. The results suggested that niclosamide has a protective role in the cell upon activation, rather than being detrimental to cell survival.

It appeared in that in the presence of niclosamide, active nitroreductases were able to grow while those with low activity weren't (Figure 3.19); the potential of this phenomenon to be exploited as a novel nitroreductase screening method was 
explored. The potential discover new nitroreductase enzymes from metagenome libraries has large implications in the use of nitroreductases as it would provide access to an even wider range of nitroreductases to work with. Functional screens of metagenome libraries are a very powerful tool to recover enzymes, and could possibly uncover previously unknown families of nitroreductases as these libraries are predicted to be comprised primarily of DNA from microbes that cannot be cultured in the lab (Vartoukian et al., 2010).

To confirm the presence of a nitroreductase in niclosamide optimisation experiments, a masked fluorophore that had been shown to be active with the majority of active nitroreductases available in the lab, FSL41, was added to the culture, the presence of fluorescence indictaed the presence of a nitroreductase. However, in order for niclosamide to be applied as a positive selection agent to detect nitroreductase expression in E. coli, it was discovered that it was necessary for the endogenous tolC gene to be removed from that E. coli strain. It was hypothesised that in the presence of this efflux regulator, niclosamide is rapidly expelled from the bacterial cell, rendering the protective effect of an expressed nitroreductase redundant. It goes without saying, that deletion of endogenous nitroreductases from the host genome is also necessary to maximise the sensitivity of the screening system.

These are just preliminary optimisation steps as a proof of principle towards the generation of a novel system to identify new nitroreductases, or for a use in directed evolution. Future work could involve the screening of libraries available within the lab, or as a novel tool for directed evolution to decrease the efficacy of a nitroreductase with a nil bystander antibiotic for a better dual ablation opposing activation profile. This was demonstrated by validation experiments in liquid and solid media, which showed niclosamide to be a powerful tool for detection of nitroreductase activity. 


\section{Masked Fluorophores}

\subsection{Introduction}

Having demonstrated in both bacterial and mammalian cells the ability of nitroreductases to metabolise nitro-quenched nil bystander antibiotics, we sought to also utilise the properties of the nitroreductases for activation of masked fluorophores. The ability of nitroreductases to activate both types of compound simultaneously would enable the development of a multiplex reporter/ target cell ablation system. This system would allow reporting of targeted cells through fluorescence visualisation, and temporal and spatial over targeted cell ablation.

The masked fluorophores used in this study are a mixture of bioreductive trigger, and nitro switch molecules as described in section 1.6. The nitro-switch quenched fluorophores have a nitro group that, upon reduction by a nitroreductase, activates the fluorescence of the fluorophore (Figure 1.7). The bioreductive trigger quenched fluorophores were developed based on the activation mechanism of other known bioreductive molecules; reduction by a nitroreductase results in an intramolecular fragmentation at a linker group which consequently results in the activation of the fluorophore (Figure1.8). In either case, like nil bystander antibiotics, these molecules are able to exploit the capabilities of nitroreductases to reduce nitro-quenched compounds, revealing their fluorescent properties only after reduction in the presence of nitroreductases.

Teaming these with the nil bystander antibiotics would enable a system to be used in developmental and regenerative biology, whereby specific cells could be fluoresced and ablated temporally and spatially. Using this dual reporter/ ablation multiplex system, one nitroreductase would be able to activate both a nil bystander antibiotic and a masked fluorophore, while a different nitroreductase would be incapable of activating that nil bystander and masked fluorophores, but would be able to activate a different nil bystander antibiotic and masked fluorophores, which the first nitroreductase would be incapable of activating (Figure 1.11). 
This would mean that the cells containing the nitroreductase would be able to be visualised upon the addition of the fluorophores, and subsequently, the addition of the nil bystander antibiotic would ablate the cells, leaving no fluorescence visible.

To test this theory, an E. coli $6 \mathrm{KO}$ library was generated, comprising of 23 nitroreductases that had potential as candidates in the dual ablation system. These nitroreductases were then screened against a range of masked fluorophores that were available in the lab to identify their activation. The masked fluorophores to be screened were chosen based on earlier screening work undertake by a previous $\mathrm{PhD}$ student, Dr Horvat; those that had shown the most suitable activation profiles were rescreened against the new 6KO library.

Again, for this system to be relevant, it needed to work in mammalian cells. Once the system had been shown to be viable in bacterial cells, the nitroreductases were then transfected into human cell lines for further experimental work.

\subsubsection{Masked fluorophores}

The nitro-quenched masked fluorophores used in this study were developed by Dr Jeff Smaill, Dr Amir Ashoorzadeh, Dr Adam Patterson, and other researchers at the Auckland Cancer Society Research Centre and can be seen in table 2.1

The excitation/ emission wavelengths of these molecules vary, producing a wide range of emitted colours. The masked fluorophores selected for use in this study fluoresce either blue, green, or red.

\subsubsection{Fluorescent reporter proteins}

Currently, fluorescence reporter proteins are the standard molecular imaging tool. The green fluorescent protein (GFP) was the first to be used in this manner. It was discovered in the early 1960s when researchers studying the bioluminescent properties of Aequorea victoria jellyfish isolated a blue-light-emitting bioluminescent protein called aequorin, together with another protein that was eventually named the green-fluorescent protein (Kremers et al., 2011). Prasher et al., (1992) first cloned the 
GFP gene in 1992, after which it was used in various reporter experiments from tracking gene expression in bacteria to that of higher eukaryotes. GFP has since been engineered to yield a wide range of useful coloured fluorescing proteins, including blue, cyan, and yellow mutants (Kremers et al., 2011). Further to this, fluorescent proteins from a broad range of other species have also been identified, expanding the fluorescent colours available through to the far-red spectrum (Kremers et al., 2011). As a group, these are loosely referred to as fluorescent proteins (FPs).

Advances in fluorescent probes with red-shifted spectra has resulted in the creation of red fluorescent proteins with enhanced spectral and biochemical characteristics $(\mathrm{Wu}$ et al., 2011). Reduced auto-fluorescence (which often interferes with GFP readings), low light scattering, and minimal absorbance at the longer wavelengths make red fluorescent proteins (RFP) superior probes for cell, tissue, and whole-body imaging, as the fluorescence is able to be visualised through several millimetres of mammalian tissue (Wu et al., 2011).

\subsubsection{Dual ablation/ reporter system in developmental biology}

Fluorescent proteins are commonly used as reporter components in developmental and regenerative biology. Reporting on the target cell population is essential for verification of cell ablation. Techniques employing the use of a fluorescent protein/ nitroreductase fusion protein under the expression of a chosen promoter have been utilised in a number of studies in the developmental and regenerative biolgy field(Curado et al., 2007; Kaya et al., 2012; Pisharath et al., 2009; White and Mumm 2013). It is a promising technology as no other inducible knock out system has been capable of such precision.

The characteristic promiscuity of nitroreductases allows them to reduce nitroquenched compounds as well as prodrugs. The library of nitro-quenched masked fluorophores available in the lab (table 2.1), have previously been shown to be activated by a variety of nitroreductases in the 58 nitroreductase core library. We propose to utilise this ability by using the activated fluorescence as a reporter for targeted cell ablation in cell populations carrying a nitroreductase of choice. This will eliminate the need for a fluorescent protein/ nitroreductase fusion protein, as only the 
nitroreductase will be needed to be driven by a promoter for reporter and ablation properties. The ability to use just one nitroreductase to induce the properties of fluorescence, and ablation in a target population, make it a simpler and more flexible technology.

\subsubsection{KO library}

High levels of background fluorescence observed are observed in the SOS-R2 strain due to activation of the masked fluorophores by endogenous nitroreductases; as such, plasmids expressing any nitroreductase candidates to be screened fluorescently in bacteria first had to be transformed into the $6 \mathrm{KO}$ strain. The nitroreductases chosen to be transformed into the $6 \mathrm{KO}$ strain were those which had been identified through the growth inhibition screening process to have, or the potential to have, opposing activation profiles with nil bystander antibiotics. Due to this selection criterion, this set of nitroreductases is very heavily weighted to the $n f s A$ family; while many NfsA enzymes were capable of activating a subset of nil bystander antibiotic candidates, the $n f s B$ enzymes had a much broader range of activation, and there were only a few that activated a subset of nil bystander antibiotic candidates that were not readily activated by members of the $n f s A$ family.

Table 4.1 23 nitroreductase 6KO library

\begin{tabular}{|r|l|l|l|l|}
\hline$\#$ & Gene & Organism & Family & $\%$ ID/Sim \\
\hline 1 & NfsA_Ec & Escherichia coli & NfsA & $100 / 100$ \\
\hline 2 & NfsA_St & Salmonella enterica & NfsA & $87 / 95$ \\
\hline 3 & NfsA_Ck & Citrobacter koseri & NfsA & $86 / 92$ \\
\hline 4 & NfsA_Kp & Klebsiella pneumoniae & NfsA & $83 / 92$ \\
\hline 5 & NfsA_Es & Enterobacter sakazakii & NfsA & $82 / 94$ \\
\hline 6 & NfsA_Ecaro & Erwinia carotovara & NfsA & $65 / 82$ \\
\hline 7 & NfsA_Vf & Vibrio fischeri & NfsA & $52 / 68$ \\
\hline 8 & NfsA_Vv & Vibrio vulnificus & NfsA & $51 / 65$ \\
\hline 9 & Frp1_Vh & Vibrio harveyi & NfsA & $51 / 65$ \\
\hline 10 & NfrA_Bs & Bacillus subtilis & NfsA & $39 / 62$ \\
\hline 11 & NfsA_Lw & Listeria welshimeri & NfsA & $41 / 60$ \\
\hline 12 & NfsA_Li & Listeria innocua & NfsA & $39 / 60$ \\
\hline 13 & NfsA_Bco & Bacillus coagulans & NfsA & $38 / 60$ \\
\hline 14 & NfsA_Np & Nostoc punctiforme & NfsA & $38 / 59$ \\
\hline 15 & NfsA_Bth & Bacillus thuringiensis & NfsA & $35 / 58$ \\
\hline 16 & NfsA_Lsak & Lactobacillus sakei & NfsA & $40 / 57$ \\
\hline
\end{tabular}




\begin{tabular}{|r|l|l|l|l|}
17 & NfsA_Pp & Pseudomonas putida & NfsA & $35 / 57$ \\
\hline 18 & YcnD_Bs & Bacillus subtilis & NfsA & $35 / 56$ \\
\hline 19 & NfsB_Ec & Escherichia coli & NfsB & $100 / 100$ \\
\hline 20 & NfsB_Vv & Vibrio vulnificus & NfsB & $61 / 75$ \\
\hline 21 & NfsB_Pp & Pseudomonas putida & NfsB & $52 / 71$ \\
\hline 22 & NfsB_Vh & Vibrio harveyi & NfsB & $30 / 48$ \\
\hline 23 & YfkO_Bs & Bacillus subtilis & NfsB & $25 / 47$ \\
\hline
\end{tabular}

\subsubsection{Aims}

The aims of this chapter were to i) Illustrate the activation profiles of the masked fluorophores with members of the 6KO 23 nitroreductase library, identifying pairs of different coloured masked fluorophores/ nitroreductases which show opposing activation profiles ii) Combine the results from (i) with results observed in chapter 3 of nil bystander antibiotics/ nitroreductases combinations with opposing activation profiles to create a multiplex reporter/ targeted cell ablation system in mixed population cells in the $6 \mathrm{KO}$ strain; iii) Translate the results observed in bacterial cells into human cell lines for proof of principle of use in eukaryotic models.

\subsection{Results}

\subsubsection{Fluorescent screening in bacterial cells}

Dr Horvat had previously run fluorescent screens with all 54 of the masked fluorophores available in the lab against a number of nitroreductases from the full 58 nitroreductase library in the $6 \mathrm{KO}$ strain, and generated a heat-map from the data obtained. The masked fluorophores that looked to be the most promising in terms of opposing activation profiles of different coloured fluorescence were identified using her heat-map. These 16 masked fluorophores (Table 2.2) were screened against the 23 nitroreductase $6 \mathrm{KO}$ library by fluorescence screening as described in 2.9 .4 and a heat-map of the results was generated (Figure 4.1). The results of this screening confirmed the activation profiles that Dr Horvat had observed, while adding additional information for some NfsA family members that were not available during Dr Horvat's research. The results can be seen in figure 4.4. 
While a lot of masked fluorophores are either active with the majority of nitroreductases, or show little to no activity with the majority of the nitroreductases, it is clear to see that some masked fluorophores are more active with a related group of nitroreductases. For example, the blue fluorophores FSL162, FSL163, FSL175, FSL176 can clearly be seen to have high activity with the majority of the NfsB enzymes present, while there is little to no activity with the NfsA family enzymes. What is more interesting however, is the clear preference of the activation of the two green fluorophores, FSL76 and FSL150 with the NfsA family enzymes, while the red masked fluorophore, FSL178, appears to have a clear activation preference with the $\mathrm{NfsB}$ family fluorophores. These observations make a good basis for future experimentation of a multiplex reporter system. 


\begin{tabular}{|c|c|c|c|c|c|c|c|c|c|c|c|c|c|c|c|c|}
\hline & 25 & 48 & 59 & 61 & 95 & 111 & 162 & 163 & 169 & 170 & 171 & 175 & 176 & 76 & 150 & 178 \\
\hline NfsA_Ec & 2326 & 14421 & 11798 & 12053 & 4747 & 14114 & 2835 & 2675 & 7905 & 1362 & 16430 & 398 & 448 & 4399 & 2554 & 0 \\
\hline NfsA_St & 4285 & 11087 & 12764 & 13672 & 9276 & 15174 & 2783 & 1394 & 6754 & 3787 & 15873 & 865 & 986 & 2623 & 3286 & 116 \\
\hline NfsA_Ck & 4865 & 3987 & 12937 & 13654 & 3789 & 2876 & 2364 & 1784 & 8753 & 8992 & 3027 & 385 & 249 & 2098 & 649 & 5 \\
\hline NfsA_Kp & 789 & 12766 & 13654 & 12998 & 4989 & 16389 & 789 & 657 & 7584 & 376 & 3762 & 379 & 346 & 3004 & 2868 & 132 \\
\hline NfsA_Es & 797 & 3971 & 13199 & 10264 & 577 & 17498 & 1124 & 1240 & 8383 & 4609 & 22993 & 983 & 826 & 3120 & 394 & 0 \\
\hline NfsA_Ecaro & 781 & 4973 & 13284 & 12066 & 50 & 17123 & 958 & 1748 & 6576 & 3139 & 19959 & 863 & 953 & 2544 & 439 & 0 \\
\hline NfsA_Vf & 4296 & 3627 & 654 & 453 & 11976 & 643 & 876 & 2098 & 8450 & 412 & 16938 & 374 & 401 & 2435 & 735 & 8 \\
\hline NfsA_Vv & 7893 & 9873 & 9765 & 8754 & 3892 & 9866 & 1678 & 2763 & 6890 & 8726 & 17986 & 484 & 389 & 2098 & 2657 & 157 \\
\hline Frp1_Vh & 876 & 9862 & 9862 & 7845 & 4091 & 8753 & 567 & 548 & 8456 & 2093 & 2864 & 386 & 397 & 2140 & 2186 & 6 \\
\hline NfrA_Bs & 638 & 8762 & 356 & 463 & 436 & 367 & 687 & 1409 & 7854 & 378 & 19863 & 487 & 635 & 3264 & 986 & 20 \\
\hline NfsA_Lw & 4547 & 4203 & 11261 & 10744 & 2097 & 1346 & 879 & 1596 & 4154 & 2035 & 21264 & 115 & 176 & 3814 & 328 & 0 \\
\hline NfsA_Li & 4793 & 4098 & 11466 & 10848 & 2919 & 1842 & 1909 & 1117 & 7421 & 9796 & 20249 & 497 & 800 & 3513 & 482 & 0 \\
\hline NfsA_Bco & 652 & 2567 & 12683 & 11875 & 2987 & 3291 & 754 & 647 & 3962 & 3092 & 3674 & 476 & 376 & 2987 & 364 & 7 \\
\hline NfsA_Np & 875 & 3676 & 15638 & 14678 & 355 & 16739 & 354 & 537 & 4672 & 456 & 19853 & 326 & 475 & 1537 & 482 & 0 \\
\hline NfsA_Bth & 763 & 4437 & 2859 & 13780 & 653 & 2876 & 577 & 378 & 2498 & 3289 & 2075 & 397 & 377 & 354 & 427 & 100 \\
\hline NfsA_Lsak & 659 & 2335 & 13787 & 13247 & 322 & 12973 & 584 & 436 & 3974 & 356 & 254 & 6087 & 765 & 325 & 367 & 34 \\
\hline NfsA_Pp & 4457 & 10973 & 12840 & 12893 & 4921 & 12665 & 765 & 856 & 9836 & 4209 & 17634 & 421 & 633 & 454 & 1758 & 80 \\
\hline YcnD_Bs & 857 & 276 & 367 & 456 & 265 & 3782 & 349 & 453 & 543 & 234 & 378 & 532 & 329 & 493 & 375 & 384 \\
\hline NfsB_Ec & 687 & 8275 & 9836 & 12896 & 654 & 14672 & 4378 & 4326 & 10897 & 356 & 16397 & 9635 & 3987 & 264 & 653 & 289 \\
\hline $\mathrm{NfsB}_{-} \mathrm{Vv}$ & 9635 & 7626 & 17223 & 13179 & 6138 & 16662 & 12063 & 9017 & 12650 & 4971 & 18200 & 5095 & 3286 & 1118 & 387 & 591 \\
\hline NfsB_Pp & 3362 & 8546 & 18572 & 15523 & 2515 & 19280 & 3903 & 7954 & 12387 & 3769 & 21319 & 3127 & 3531 & 80 & 548 & 220 \\
\hline NfsB_Vh & 3873 & 2989 & 452 & 14563 & 356 & 463 & 10758 & 8025 & 11866 & 643 & 20186 & 235 & 327 & 120 & 742 & 9 \\
\hline YfkO_Vh & 2789 & 6532 & 3782 & 3267 & 245 & 14097 & 3094 & 645 & 10973 & 237 & 20754 & 345 & 266 & 352 & 485 & 325 \\
\hline
\end{tabular}

Figure 4.1 Heat-map of 23 nitroreductase 6KO library with different masked fluorophores. The heat-map was generated from fluorescence averages from 5

independent assays. Fluorescence is expressed as the raw fluorescent units measured after subtraction of endogenous auto-fluorescence observed in the empty plasmid control strain. All fluorophores were added to a final concentration of $50 \mu \mathrm{M}$, and fluorescence was measured at one of three different excitation/ emission settings according to the emission colour of the unmasked fluorophore; red fluorescence (ex 645/ em 660), green fluorescence (ex 405/ em 585), or blue fluorescence (ex 355/ em 460). The colour of the cells in the first row indicates the colour of fluorescence that each unmasked fluorophore emits. In this heat-map, the green squares denote nitroreductase enzymes that have high activity with the given nil bystander antibiotic, orange some activity, and red no activity. For high, activity, some activity, and low activity ranges were specified as follows; blue fluorescence >5000, 1000-4999, >1000; green fluorescence >2000, 1000-1999, >1000; red fluorescence >200, 100-199, >100 respectively. 


\subsubsection{Combined fluorescence reporting and cell ablation}

Initial experiments to demonstrate dual fluorescence and ablation capabilities were undertaken using the nitroreductases that showed promising opposing activation profile combinations in chapter 3. As FSL41 has been shown previously by Dr Horvat to be highly active with most nitroreductases, this masked fluorophore was used to identify the presence of nitroreductases during the initial optimisation.

Day cultures of $200 \mu \mathrm{L}$ were inoculated with $15 \mu \mathrm{L}$ of an overnight culture and grown for three hours prior to the addition of $25 \mu \mathrm{M}$ FSL41. After a further four hour incubation period, fluorescence was recorded qualitatively on the UV transilluminator as illustrated in Figure 4.2. In parallel, replicate cultures were treated at the $3 \mathrm{~h}$ timepoint not only with $25 \mu \mathrm{M}$ FSL41, but also with the nil bystander antibiotic they had demonstrated high activity with in figure 3.6. Again, fluorescence and turbidity were measured four hours post-challenge. Quantitative analysis on the Enspire 2300 Multiplate Reader confirmed the presence or absence of fluorescence and cell density (not shown).

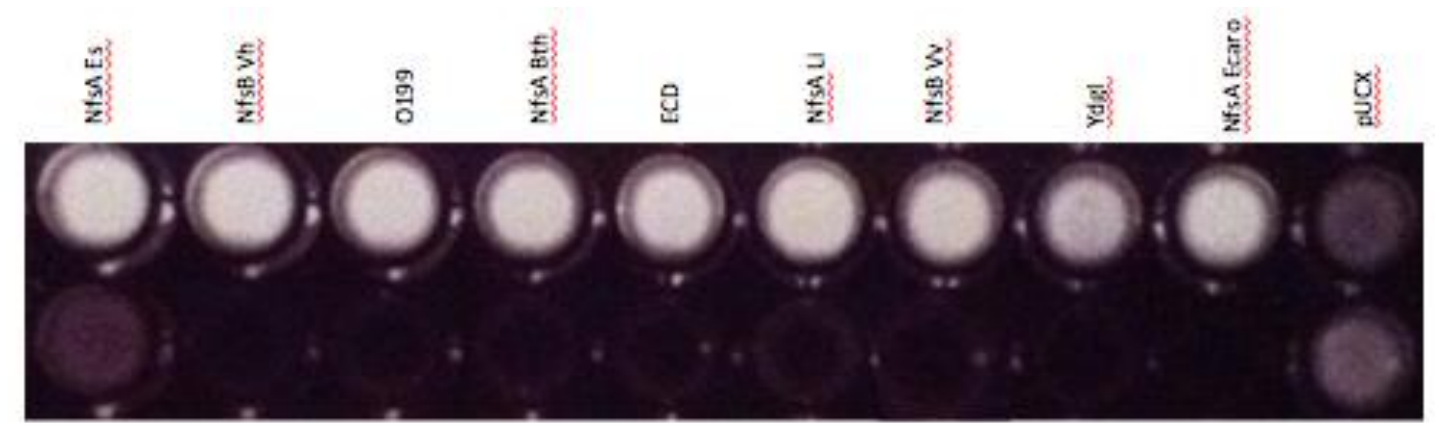

Figure 4.2 UV Trans-illuminator image of FSL41-treated cultures of $E$. coli $6 \mathrm{KO}$ over-expressing different candidate nitroreductases, with (top) or without (bottom) antibiotic challenge. Fluorescence was visualised directly on a UV trans-illuminator $4 \mathrm{~h}$ post-addition of FSL41. The top row shows the unmasking of FSL41 fluorescence by cultures of $6 \mathrm{KO}$ cells over-expressing candidate nitroreductases (as labelled), in the absence of antibiotic challenge. In contrast, the bottom row shows the absence of fluorescence when a strongly activated nil-bystander antibiotic is present as well as FSL41 (Nil bystander antibiotics in each well from left to right are as follow; EF5 (130 $\mu \mathrm{M})$, metronidazole $(50 \mu \mathrm{M})$, EF5 $(130 \mu \mathrm{M})$, F-misonidazole $(130 \mu \mathrm{M})$, tinidazole $(75 \mu \mathrm{M})$, tinidazole $(75$ $\mu \mathrm{M})$, metronidazole $(50 \mu \mathrm{M})$, metronidazole $(50 \mu \mathrm{M})$, tinidazole $(75 \mu \mathrm{M})$, metronidazole $(50 \mu \mathrm{M})$ (Here, the pUCX empty plasmid control strain was challenged with metronidazole; but has previously been shown to be resistant to all nil bystander antibiotics at the concentrations used).

These cultures were also imaged using a confocal microscope. After the $4 \mathrm{~h}$ FSL41 incubation period, samples from each culture were mixed at a 1:1 ratio with $4 \%$ paraformaldehyde (to fix the bacteria) before being placed on a microscope slide. An 
Olympus FV1000 confocal microscope with 100x lens was used to image each slide.

Settings for each laser remained the same between samples of the same excitation wavelength. Representative results for the empty plasmid control strain, and for the NfsB_Vh over-expressing strain in the absence or presence of metronidazole challenge, are depicted in Figures 4.34 .4 and 4.5, respectively.

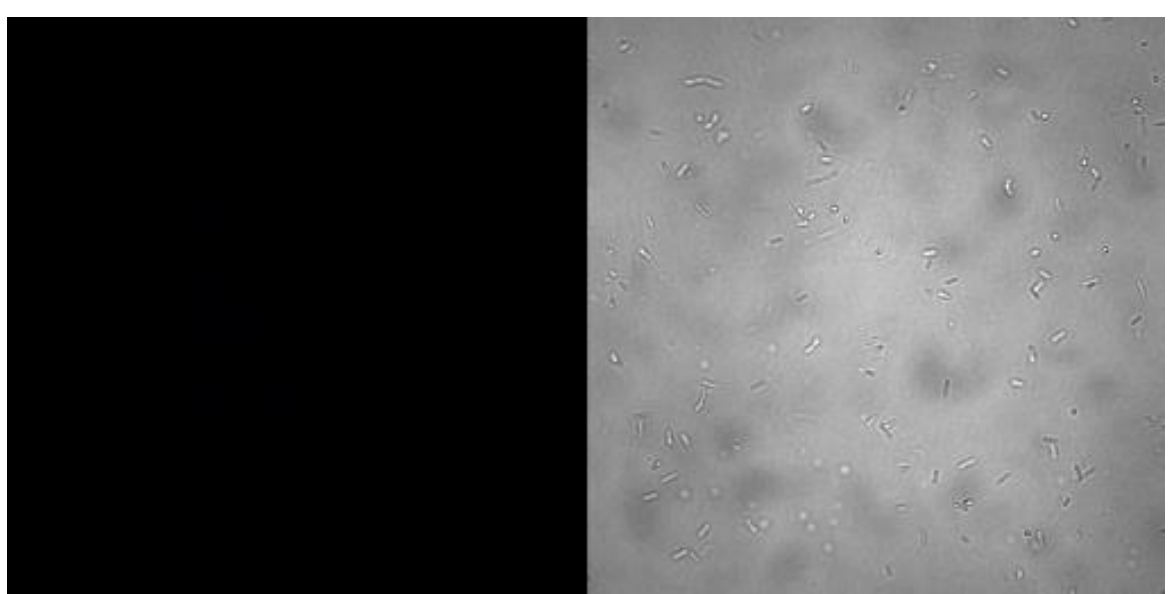

Figure 4.3 6KO E. coli empty plasmid (pUCX) control strain treated with FSL41. Cultures overexpressing empty pUCX were treated with $25 \mu \mathrm{M}$ FSL41 and incubated for $4 \mathrm{~h}$. Cultures were fixed to slides using 4\% paraformaldehyde then imaged on an Olympus FV1000 confocal microscope using the 100x lens, at settings of 405 laser 5\%, HV $700 \mathrm{~V}$, Offset 10\% and Gain 1x. The left side of this image shows no fluorescence produced, as there is no nitroreductase present to metabolise FSL41 to its active form. The right side shows the same bacteria under transmitted light.

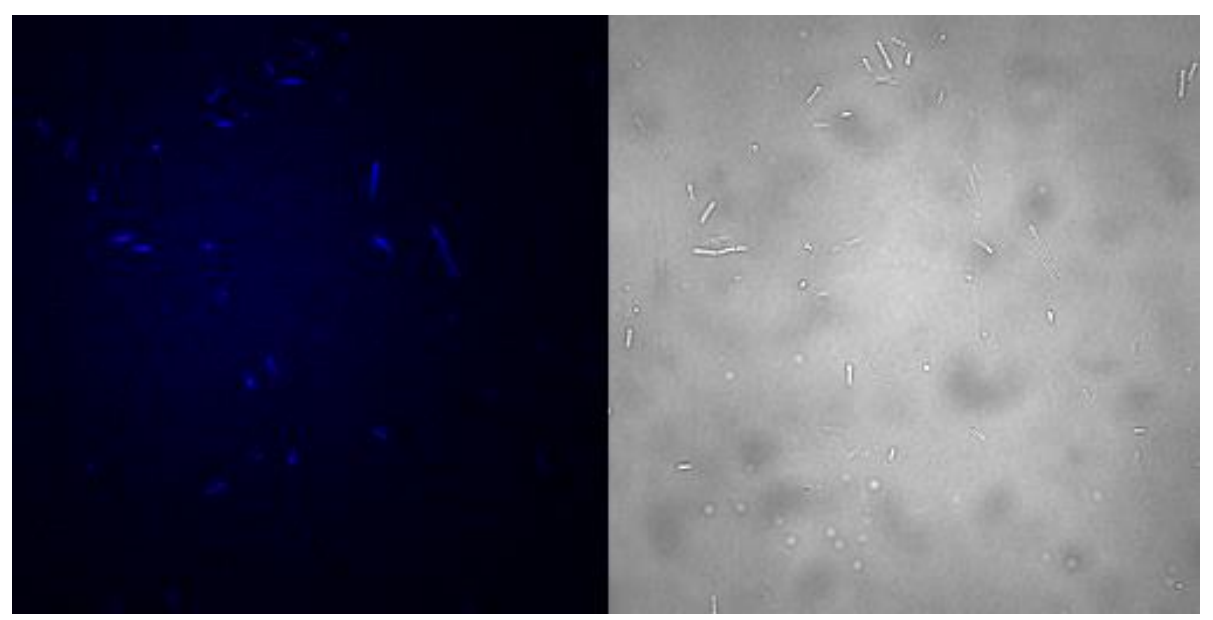

Figure 4.4 6KO $E$. coli over-expressing NfsB_Vh, treated with FSL41. Cultures over-expressing nitroreductase NfsB_Vh were treated with $25 \mu \mathrm{M}$ FSL41 and incubated for $4 \mathrm{~h}$. Cultures were fixed to slides using 4\% paraformaldehyde then imaged on an Olympus FV1000 confocal microscope using the 100x lens, 405 laser, at settings of 5\%, HV $700 \mathrm{~V}$, Offset 10\% and Gain 1x. The left side of this image shows the fluorescence produced by FSL41 being metabolised in the presence of NfsB_Vh, the right hand shows the same bacteria under transmitted light. 


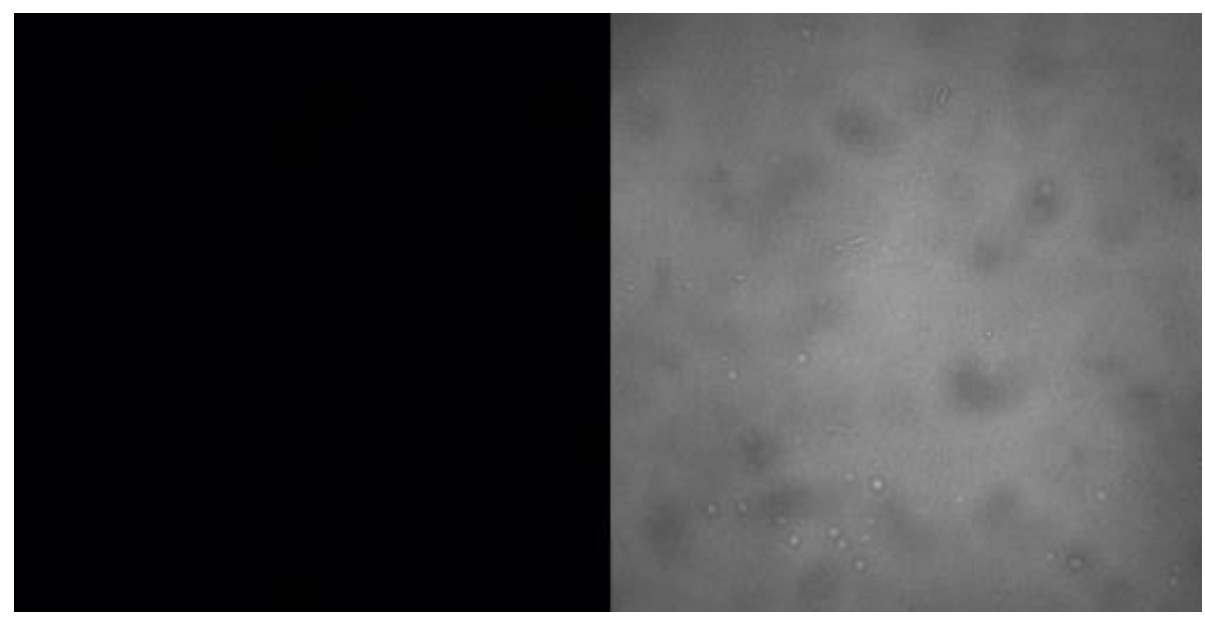

Figure 4.5 6KO E. coli over-expressing NfsB_Vh treated with FSL41 and metronidazole.

Cultures over-expressing nitroreductase NfsB_Vh were treated with $25 \mu \mathrm{M}$ FSL41 and challenged with $50 \mu \mathrm{M}$ metronidazole and incubated for $4 \mathrm{~h}$. Cultures were fixed to slides using $4 \%$ paraformaldehyde then imaged on an Olympus FV1000 confocal microscope using the 100x lens, 405 laser at settings of $5 \%$, HV $700 \mathrm{~V}$, Offset $10 \%$ and Gain 1x. The right hand side of this image shows the disappearance of fluorescence indicating that the cells are dying due to the presence of metronidazole. This is confirmed by the image on the right hand side, the bright field image of the same area, consistent with complete cell ablation.

Together, these results confirm the ability of each nitroreductase candidate to utilise a combination of nil bystanders and nil bystander antibiotics as an effective reporter/ ablation system in $E$. coli cells. Whereas fluorescence is clearly visible in nitroreductase-expressing cell cultures treated with FSL41 in the absence of antibiotic, post-antibiotic challenge no fluorescence is seen. The OD600 measurements taken on the Enspire 2300 Multiplate Reader confirm that the loss of fluorescence is due to ablation of the cell population (not shown).

The next step was to demonstrate that these results could be translated to eukaryotic cells. This was first tested using the stable HCT116: NfsB_Vh cell line that had previously been established by Dr Horvat (Section 3.2.1). These cells were seeded at a 5 million cell density into duplicate $35 \mathrm{~mm}$ fluorodishes and grown overnight. The following day, $150 \mu \mathrm{M}$ metronidazole was added to one of the cell populations and incubated for an hour. $25 \mu \mathrm{M}$ FSL41 was then added to both the challenged and unchallenged cell populations, which were then incubated for a further hour. The cells were then visualised on an Olympus FV1000 confocal microscope.

The results mirrored those observed in bacterial cells, with HCT116: NfsB_Vh found to be capable of activating the fluorescence of FSL41 (Figure 4.8). Upon addition of 
metronidazole, however, the fluorescence disappears due to the ablation of the cell population (Figure 4.9).

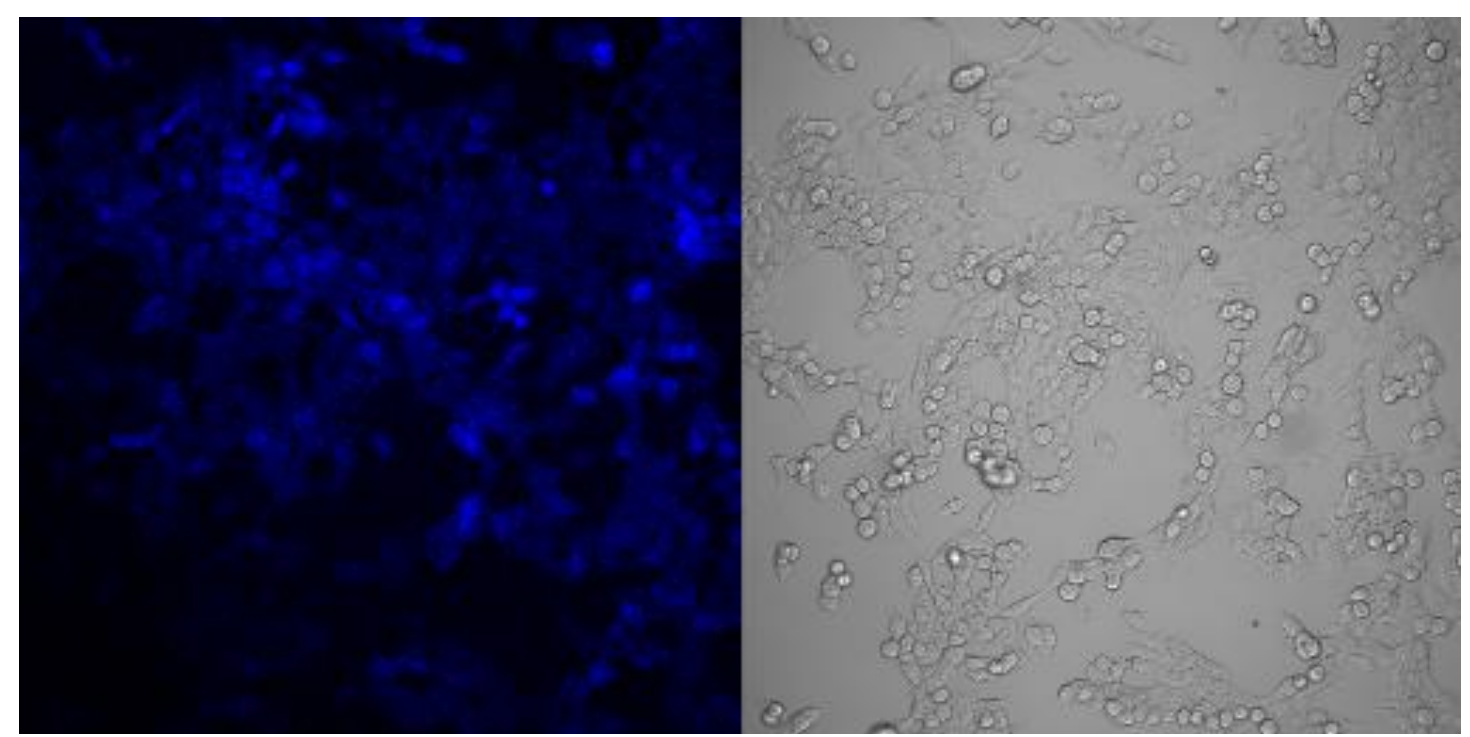

Figure 4.6 HCT116: NfsB_Vh treated with FSL41. Cultures of HCT-116 over-expressing nitroreductase NfsB_Vh were treated with $25 \mu \mathrm{M}$ FSL41 and incubated for $1 \mathrm{~h}$. Cultures were then visualised on an Olympus FV1000 confocal microscope using the 20x lens, 405 laser at settings of 5\%, HV $700 \mathrm{~V}$, Offset $10 \%$ and Gain 1x. The left hand side of this image shows the fluorescence produced by FSL41 being metabolised in the presence of NfsB_Vh, the right hand side of the image shows the same HCT116 cells under transmitted light.

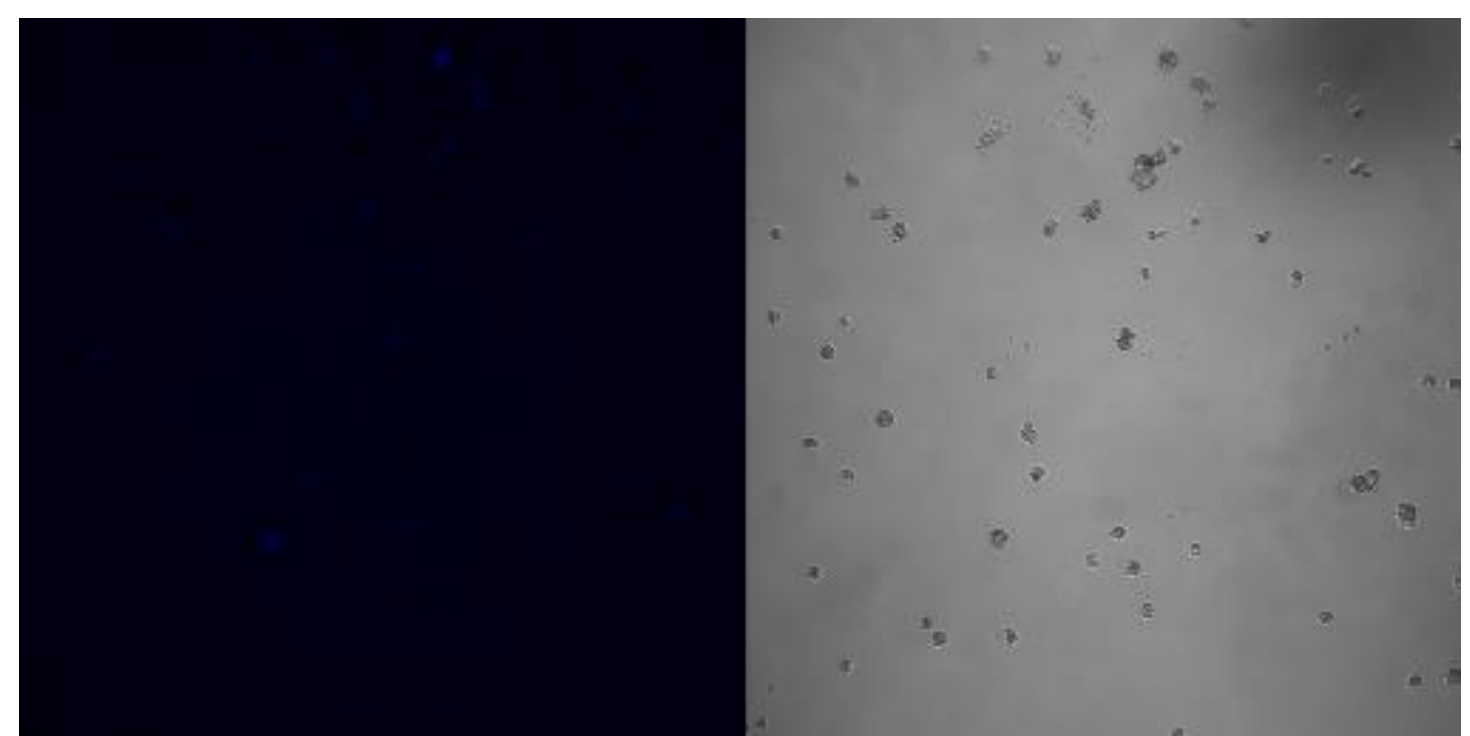

Figure 4.7 HCT116: NfsB_Vh treated with FSL41 and challenged with metronidazole. Cultures of HCT-116 over-expressing nitroreductase NfsB_Vh were treated with $5 \mu \mathrm{M}$ metronidazole and incubated for $1 \mathrm{~h} .25 \mu \mathrm{M}$ FSL41 was added and the cells were incubated for a further hour. Cultures were then visualised on an Olympus FV1000 confocal microscope using the 20x lens, 405 laser at settings of 5\%, HV $700 \mathrm{~V}$, Offset 10\% and Gain 1x. The left side of this image shows the disappearance of fluorescence indicating that the cells are dying due to the presence of metronidazole. This is confirmed by the image on the right side, the bright field image of the same area, which shows that the cell morphology has changed and they longer look healthy, as well as a reduced cell population. 


\subsubsection{Dual fluorophore screening in bacterial cells}

Following confirmation of the ability to use fluorescence reporting in a single nitroreductase cell ablation system, we next wanted to determine if selective imaging could be observed in a mixed population nitroreductase setting, with two nil bystander antibiotics and two masked fluorophores present; each nitroreductase active with only one masked fluorophore, and one nil bystander antibiotic. This was first tested in $E$. coli cells.

For the reporter system to be useful, two masked fluorophores that emitted fluorescence at different wavelengths needed to be used, to be able to distinguish between cell populations. The ideal fluorophores to be used in this setting would be green and red, as they have the longest wavelengths of the available fluorophores. This makes them the most relevant for in vivo imaging as they are the most able to penetrate tissue (Bhaumik, 2011). These two colours also don't have any crossover of wavelengths so no false positive fluorescence would be observed. This is not the case with blue and green fluorescence, for which there is some overlap in the wavelengths; this can be problematic when trying to distinguish cell populations. Moreover, blue fluorophores emit at a wavelength similar to cellular auto-fluorescence, so will have to contend with high background fluorescence (Bhaumik, 2011).

From the results of the nil bystander antibiotic and the masked fluorophore screening, two nitroreductases were selected as the best option for this analysis; NfsA_Pp and NfsB_Vv. NfsA_Pp is active with tinidazole and the green fluorophore FSL76 while $\mathrm{NfsB}_{-} \mathrm{Vv}$ is active with metronidazole and the red fluorophore FSL178. Again, NfsA_Li would have been preferred over NfsA_Pp for this analysis, but as described in section 3.2.3 it was unable to be transfected into human cell lines within the timeframe of this research. As NfsA_Pp showed very similar activation profiles to NfsA_Li with both nil bystander antibiotics and masked fluorophores, it was an acceptable substitute for these proof-of-principle experiments. Figure 4.10 shows the opposing activation profiles of NfsA_Pp and NfsB_Vv, with pairs of nil bystander antibiotics and masked fluorophores. 


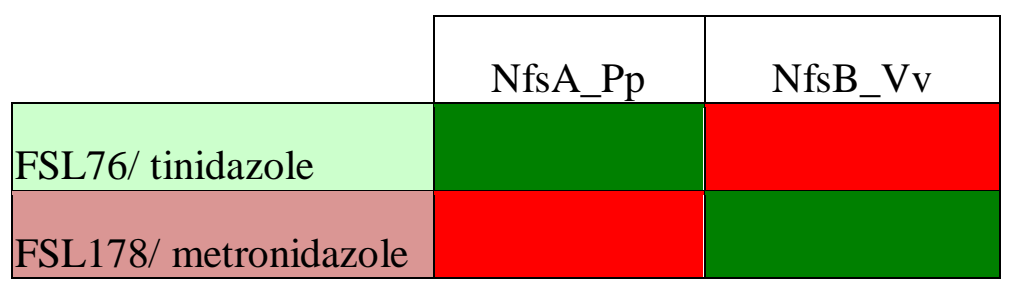

Figure 4.8 Identification of candidate nitroreductase enzymes that exhibit opposing activation profiles for two nil bystander antibiotics, and two masked fluorophores. Results summarised from combined nil bystander antibiotic and masked fluorophore screening heat-maps (Figures 3.6 and 4.1), where green indicates high activity with the compounds, while red indicates little to no activity. NfsA_Pp is capable of activating the green fluorophore FSL76, but not the red fluorophore FSL178; and is also capable of activating the nil bystander antibiotic tinidazole, but not metronidazole. NfsB_Vv shows the opposite activation profile to this, activating the red fluorophore FSL178, but not the green fluorophore FSL76; and the nil bystander antibiotic metronidazole, but not tinidazole.

\section{Replicate $200 \mu \mathrm{L}$ cultures of 6KO strains over-expressing either NfsA_Pp or}

NfsB_Vv were established by inoculation with $15 \mu \mathrm{L}$ of an overnight culture and then grown for three hours prior to the addition of masked fluorophores (final concentration of $25 \mu \mathrm{M})$. Cells were then mixed at a 1:1 ratio with $4 \%$ paraformaldehyde before being placed on a microscope slide. An Olympus FV1000 confocal microscope was used to image slides using the 100x lens. Settings for each laser remained the same between samples of the same excitation wavelength.

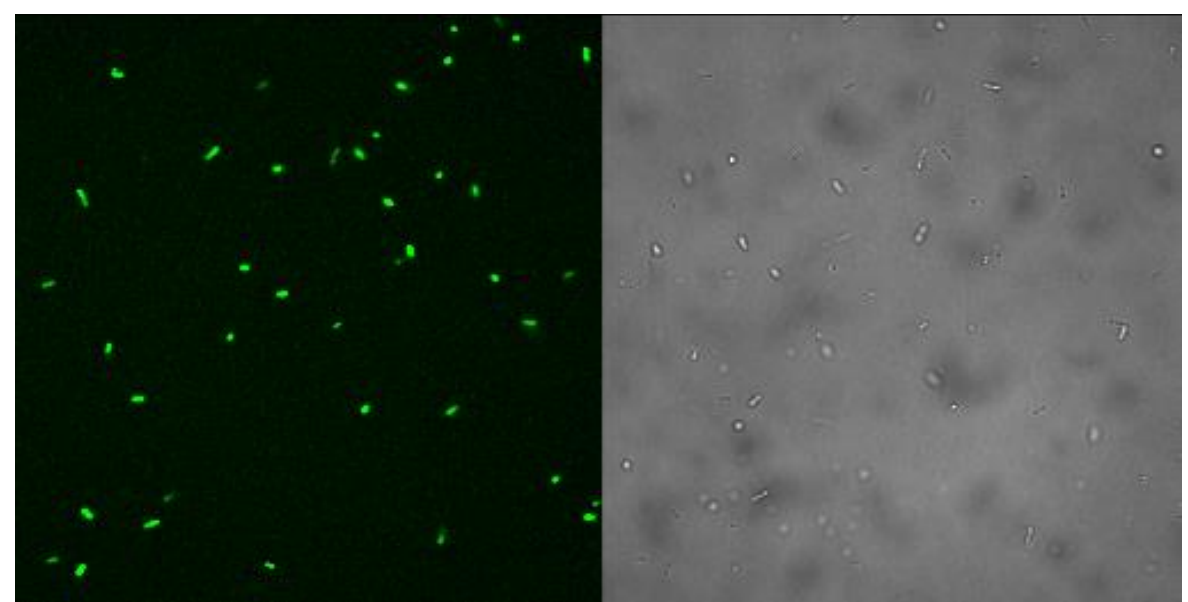

Figure 4.9 6KO E. coli over-expressing NfsA_Pp treated with FSL76. Cultures over-expressing nitroreductase NfsA_Pp were treated with $25 \mu \mathrm{M}$ FSL76 and incubated for $4 \mathrm{~h}$. Cultures were fixed to slides using 4\% paraformaldehyde then imaged on an Olympus FV1000 confocal microscope using the 100x lens, 488 laser at settings of 5\%, HV $700 \mathrm{~V}$, Offset $10 \%$ and Gain 1x. The left side of this image shows the fluorescence produced by FSL76 being metabolised in the presence of NfsA_Pp, the right hand shows the same bacteria under transmitted light. 


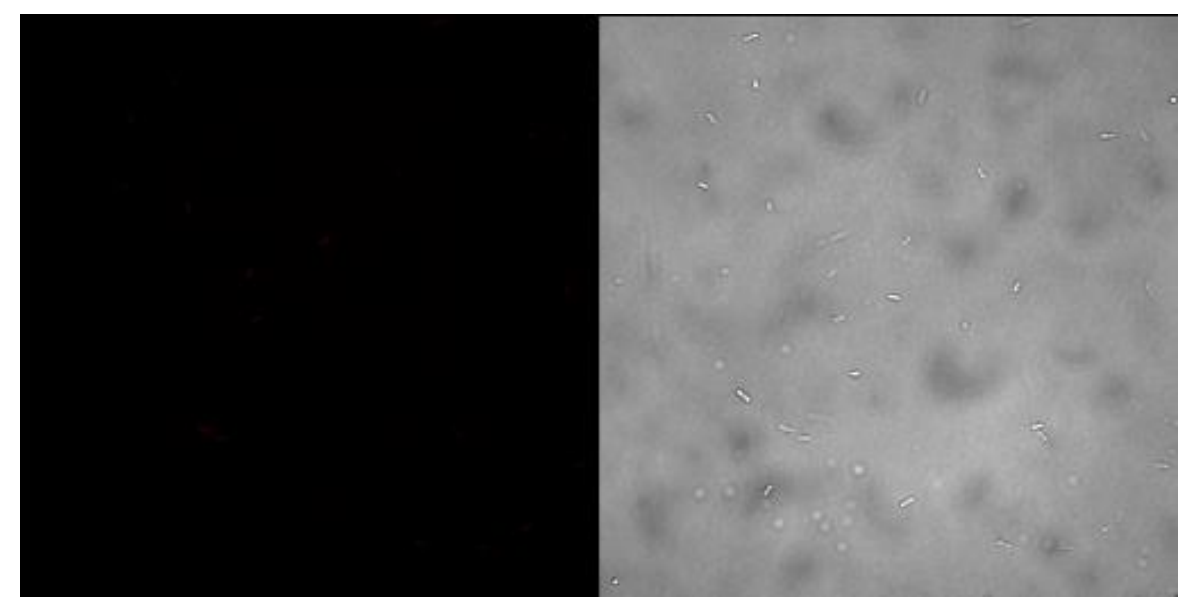

Figure 4.10 6KO E. coli over-expressing NfsA_Pp treated with FSL178. Cultures over-expressing nitroreductase NfsA_Pp were treated with $25 \mu \mathrm{M}$ FSL178 and incubated for $4 \mathrm{~h}$. Cultures were fixed to slides using $4 \%$ paraformaldehyde then imaged on an Olympus FV1000 confocal microscope using the 100x lens, 647 laser at settings of 5\%, HV 700 V, Offset 10\% and Gain 1x. The right hand side of this image shows that NfsA_Pp is incapable of activating FSL178 therefore no fluorescence is produced, the right hand shows the same bacteria under transmitted light.

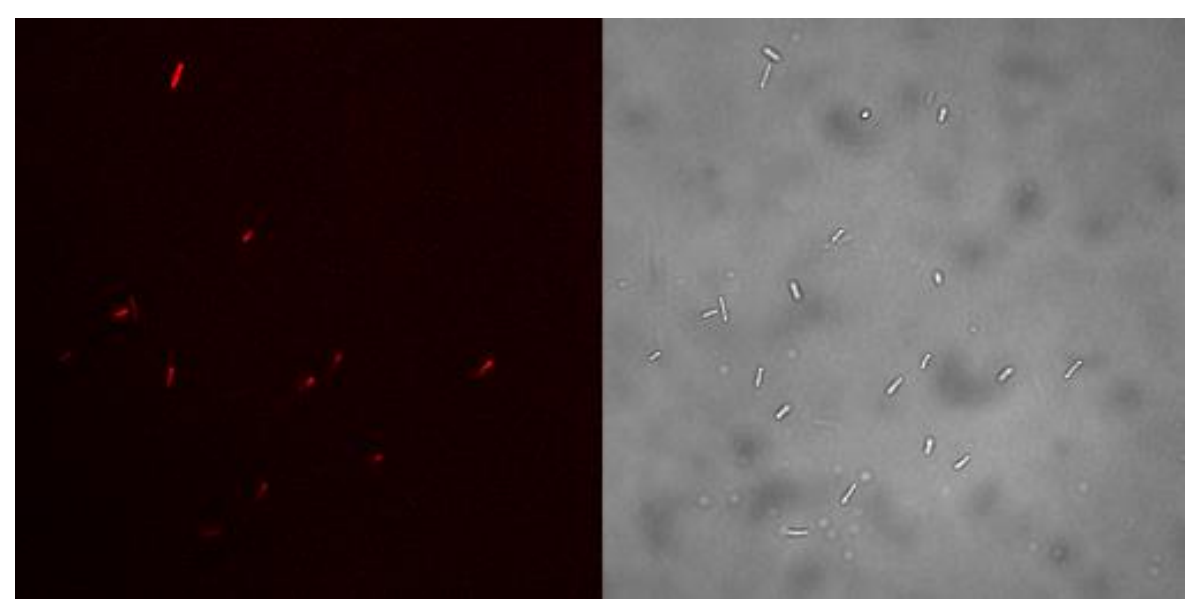

Figure 4.11 6KO E. coli over-expressing NfsB_Vv treated with FSL178. Cultures over-expressing nitroreductase NfsB_Vv were treated with $25 \mu \mathrm{M}$ FSL178 and incubated for $4 \mathrm{~h}$. Cultures were fixed to slides using 4\% paraformaldehyde then imaged on an Olympus FV1000 confocal microscope using the 100x lens 647 laser, at settings of 5\%, HV $700 \mathrm{~V}$, Offset $10 \%$ and Gain 1x. The left side of this image shows the fluorescence produced by FSL178 being metabolised in the presence of NfsB_Vv, the right hand shows the same bacteria under transmitted light.

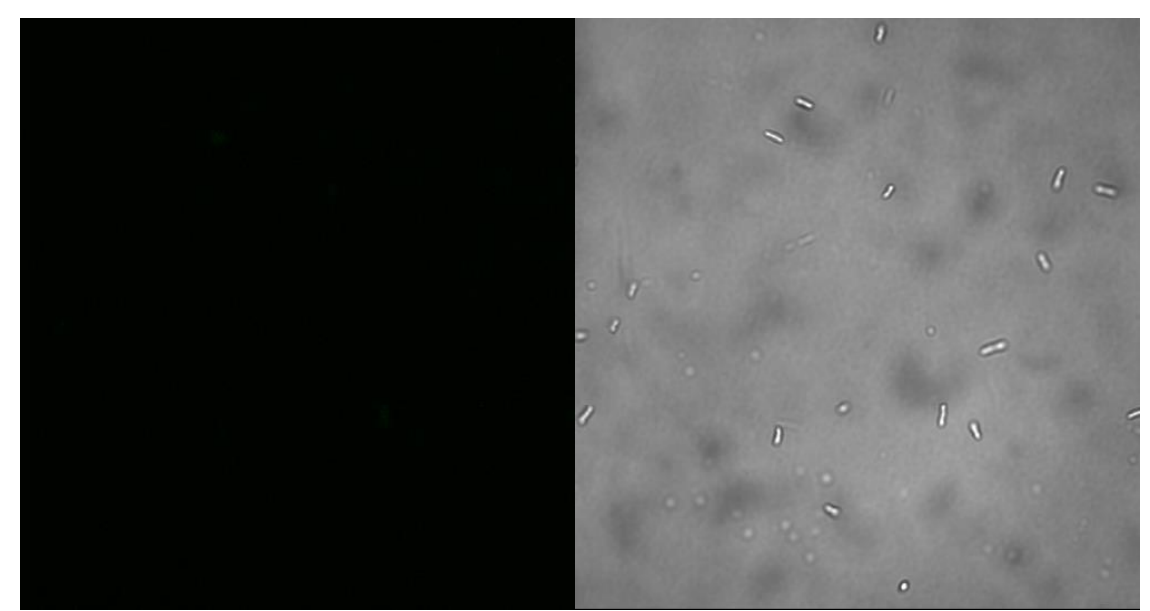

Figure 4.12 6KO E. coli over-expressing NfsB_Vv treated with FSL76. Cultures over-expressing nitroreductase NfsB_Vv were treated with $25 \mu \bar{M}$ FSL76 and incubated for $4 \mathrm{~h}$. Cultures were fixed to 
slides using 4\% paraformaldehyde then imaged on an Olympus FV1000 confocal microscope using the 100x lens 488 laser, at settings of 5\%, HV $700 \mathrm{~V}$, Offset $10 \%$ and Gain 1x. The left of this image shows that NfsB_Vv is incapable of activating FSL178 therefore no fluorescence is produced, the right hand shows the same bacteria under transmitted light.

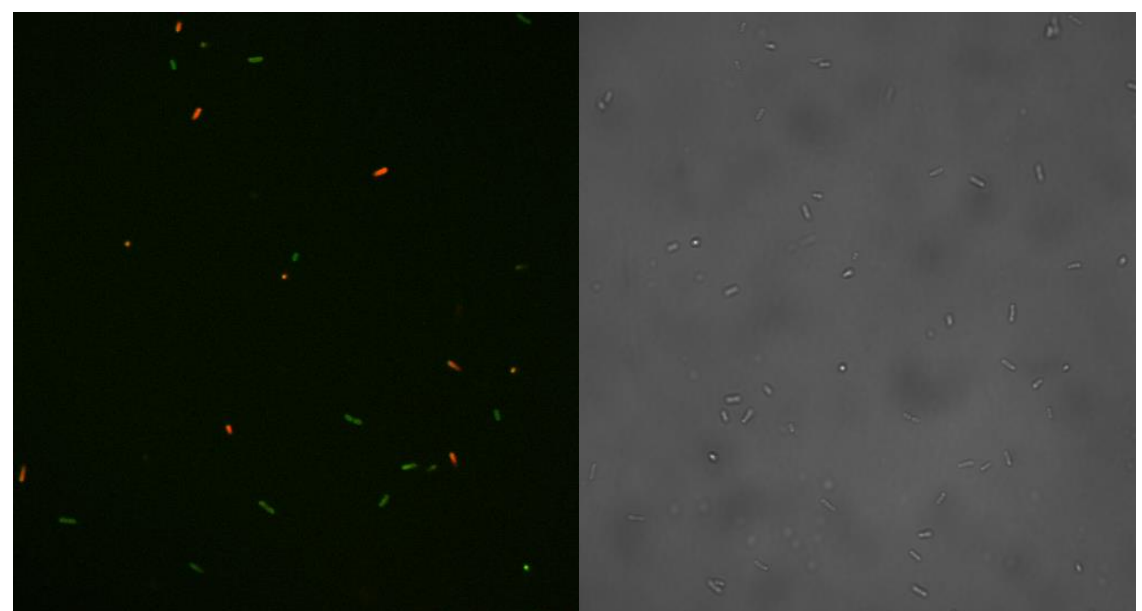

Figure 4.13 6KO $E$. coli mixed culture multiplex fluorescence imaging. A mixed population culture containing $6 \mathrm{KO} E$. coli over-expressing NfsB_Vv and NfsA_Pp was treated with both FSL76 and FSL178 and incubated for $4 \mathrm{~h}$. Cultures were fixed to slides using $4 \%$ paraformaldehyde then imaged on an Olympus FV1000 confocal microscope using the 100x lens ,488 laser at settings of 5\%, HV 700 V, Offset 10\% and Gain 1x, and 647 laser at settings of 5\%, HV 700 V, Offset 10\% and Gain 1x. The left side of this image shows two distinct cell populations, NfsA_Pp causing the green fluorescence of FSL76 and NfsB_Vv causing the red fluorescence of FSL178. The right image shows the same bacteria under transmitted light.
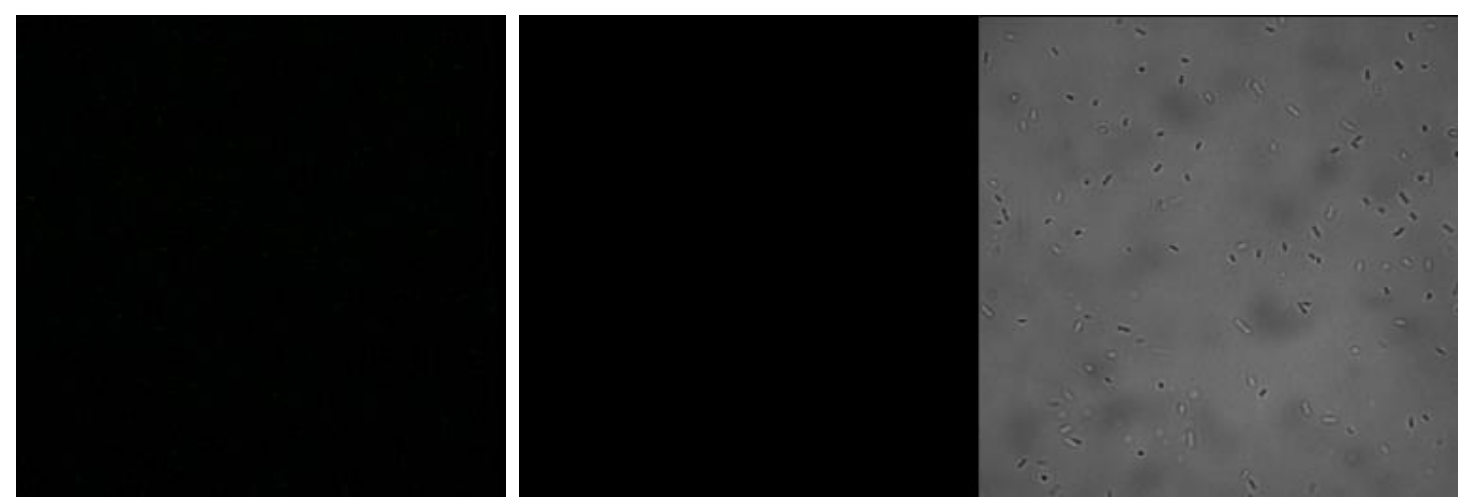

Figure 4.14 6KO $E$. coli pUCX treated with FSL76 and FSL178. Cultures over-expressing empty pUCX were treated with either $25 \mu \mathrm{M}$ FSL76 or FSL178 and incubated for $4 \mathrm{~h}$. Cultures were fixed to slides using 4\% paraformaldehyde then imaged on an Olympus FV1000 confocal microscope using the 100x lens, 488 laser at settings of 5\%, HV $700 \mathrm{~V}$, Offset 10\% and Gain 1x, for FSL76 and 647 laser at settings of 5\%, HV $700 \mathrm{~V}$, Offset $10 \%$ and Gain 1x for FSL178. The far left image and the centre image show no fluorescence produced, as there is no nitroreductase present to metabolise FSL76 (far left) or FSL178 (centre) to their active forms. The right hand shows the same bacteria under transmitted light.

The results of confocal imaging of the E.coli $6 \mathrm{KO}$ strains were consistent with the initial fluorophore screening (figure 4.1). Cultures over-expressing NfsA_Pp were capable of activating FSL76 (figure 4.9), but not FSL178 (figure 4.10). Cultures over-expressing NfsB_Vv had the opposite activation profile, activating FSL178 
(figure 4.11), but not FSL76 (4.12). A mixed population of cells over-expressing either NfsA_Pp or NfsB_Vv showed distinct fluorescence activation in individual cells (figure 4.13). A negative control with cultures over-expressing pUCX, confirmed that the presence of an over-expressed nitroreductase was necessary for the activation of fluorescence (figure 4.14).

\subsubsection{Dual fluorophore screening in human cell lines}

In mammalian cells, newly generated transfections into HEK293 cell lines were confirmed by addition of FSL41, as it had previously been shown that active nitroreductases are generally capable of reducing FSL41. All transfected cell lines were grown in DMEM + 2\% HEPES, 10\% FCS, and 2\% Penicillin/Streptomycin, 2 $\mu \mathrm{M}$ puromycin. Each transfected cell line had been subcultured at least 3 times in DMEM containing puromycin, the selection for cells containing the F279/ V5 plasmid used in the creation of Gateway ${ }^{\mathrm{TM}}$ plasmids, after the initial transfection.

For fluorescence screening in HEK293 for confirmation of stable transfected cell lines, cells were seeded at 5 million cell density into $35 \mathrm{~mm}$ fluoro dishes and left to grow overnight. The following day FSL41 was added to a final concentration of 25 $\mu \mathrm{M}$ and incubated for a further $2 \mathrm{~h}$.

After this $2 \mathrm{~h}$ time period, cells were visualised on an Olympus FV1000 confocal microscope using the 20x lens. Blue fluorescence confirmed that transfections had been successful for HEK293: NfsB_Ec (figures 4.16) HEK293: NfsB_Vv (figure 4.17), and HEK293: NfsA_Pp (figure 4.18). Negative controls with untransfected HEK293 WT cells confirmed that cells which aren't carrying a nitroreductase are incapable of activating fluorescence (figure 4.15). 


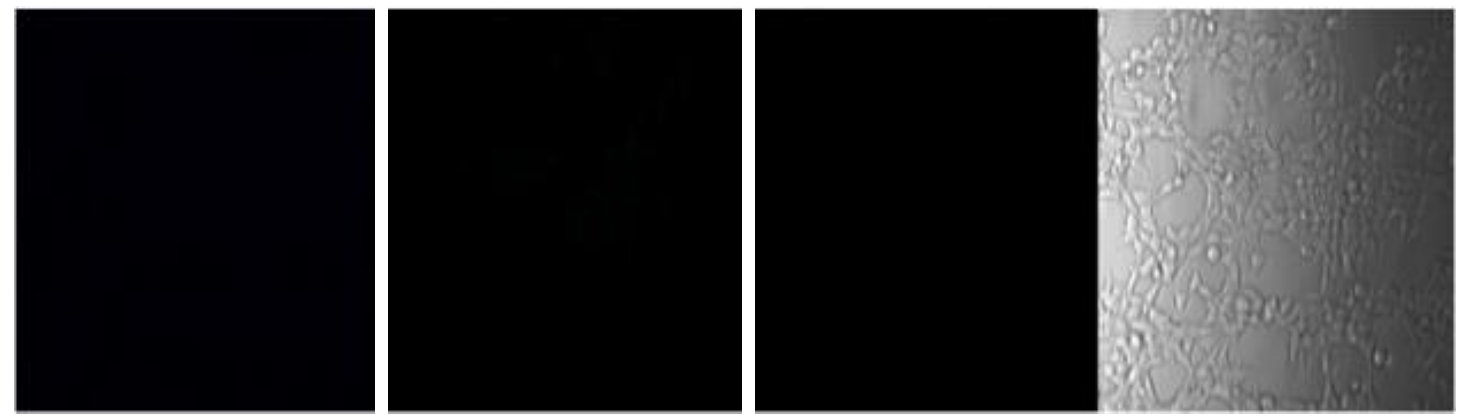

Figure 4.15 HEK293 WT cells treated with either FSL41, FSL76, or FSL178. HEK293 WT cultures were treated with $25 \mu \mathrm{M}$ of either FSL41, FSL76, or FSL178 and incubated for $1 \mathrm{~h}$. Cultures then imaged on an Olympus FV1000 confocal microscope using the 100x lens at settings of 405 laser 5\%, HV 700 V, Offset 10\% and Gain 1x, 488 laser 5\%, HV 700 V, Offset 10\% and Gain 1x, for FSL76 and 647 laser 5\%, HV 700 V, Offset 10\% and Gain 1x for FSL178. The far left image, centre left image, and the centre right image show no fluorescence, as there is no nitroreductase present to metabolise FSL41 (far left) FSL76 (centre left), or FSL178 (centre right) to their active forms. The right hand image shows the same cells under transmitted light.

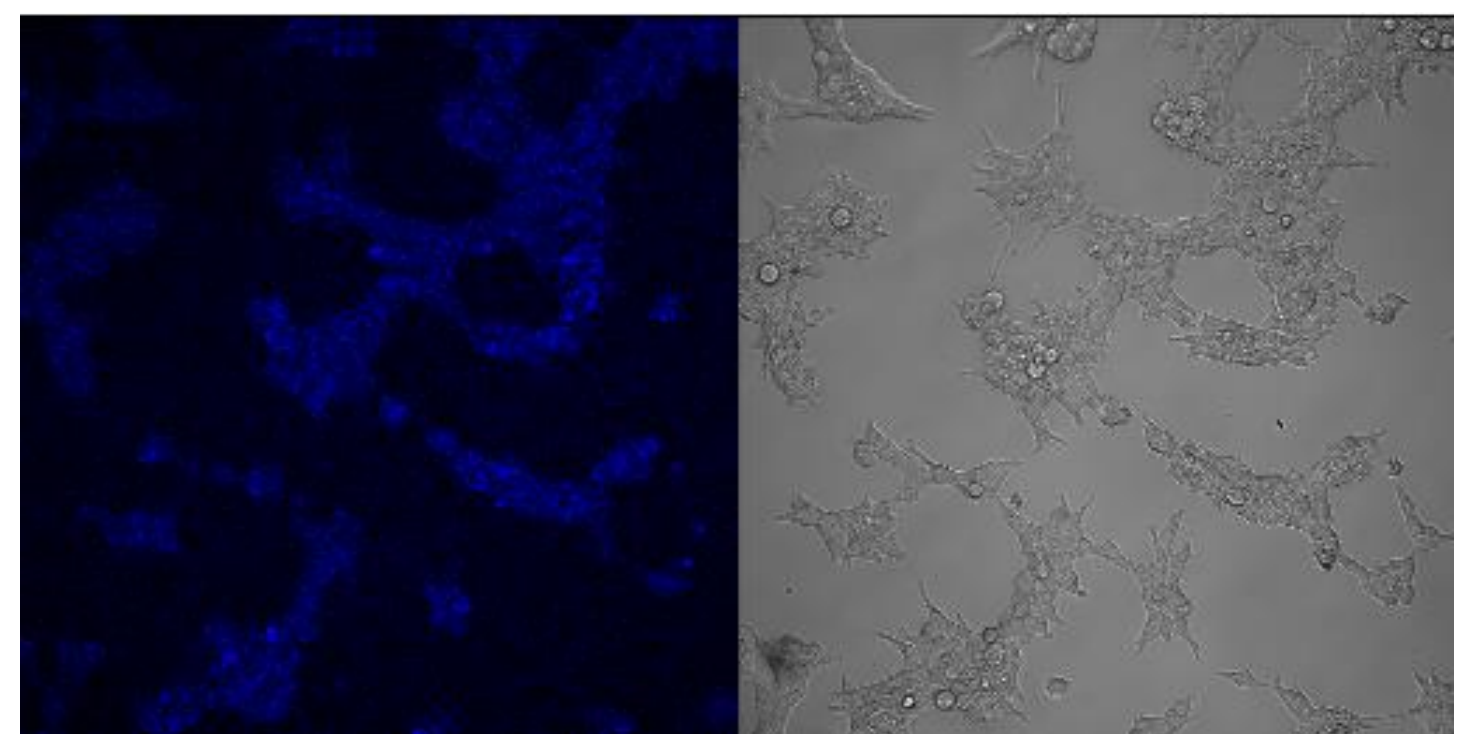

Figure 4.16 HEK293: NfsB_Ec treated with FSL41. Cultures over-expressing nitroreductase NfsB_Ec were treated with $25 \mu \mathrm{M}$ FSL41 and incubated for $1 \mathrm{~h}$. Cultures were then visualised on an Olympus FV1000 confocal microscope using the 20x lens, 405 laser at settings of 5\%, HV $700 \mathrm{~V}$, Offset $1 \%$ and Gain 1x. The left image shows the fluorescence produced by FSL41 being metabolised in the presence of NfsB_Ec, the right shows the same cells under transmitted light. The activation of FSL41 confirms that this is a stably transfected cell line. 


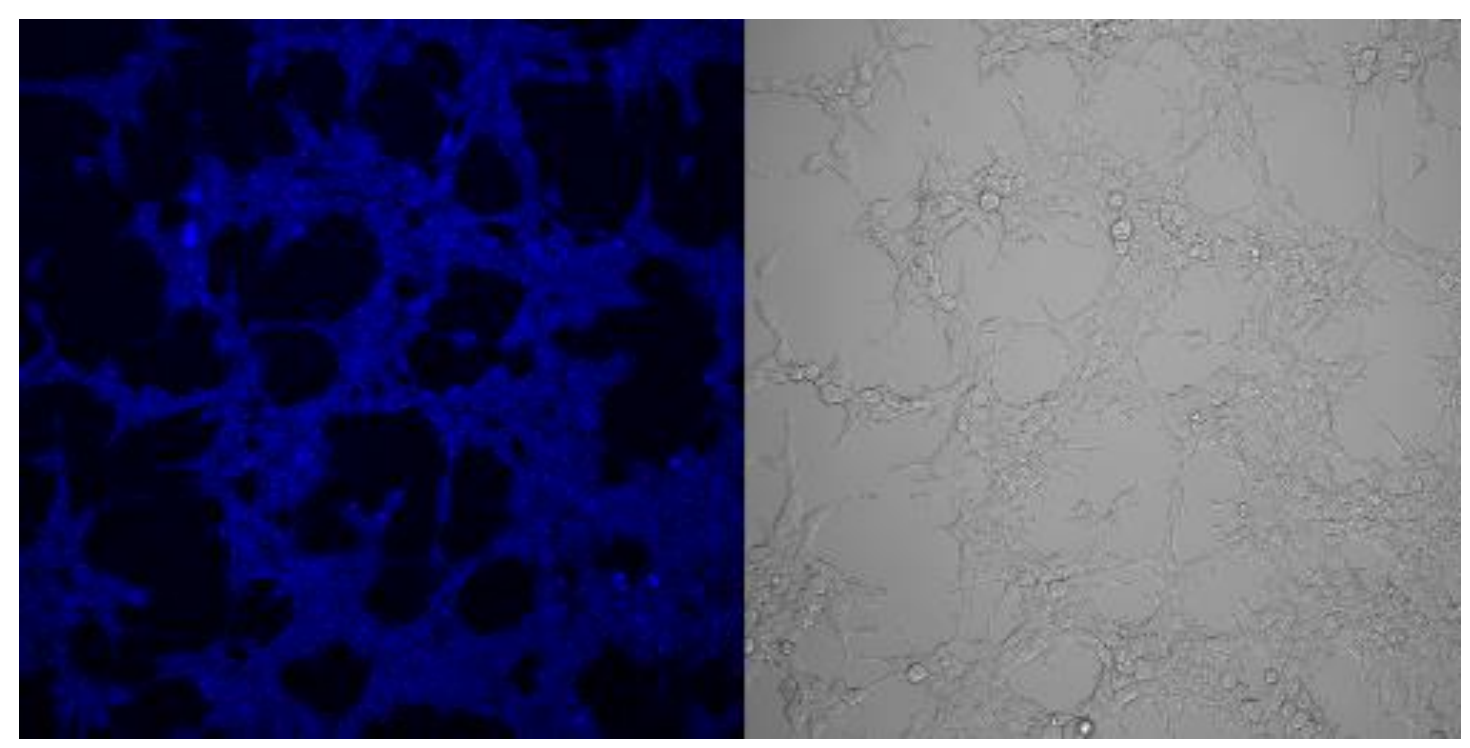

Figure 4.17 HEK293: NfsB_Vv treated with FSL41. Cultures over-expressing nitroreductase NfsB _Vv were treated with $25 \mu \mathrm{M}$ FSL41 and incubated for $1 \mathrm{~h}$. Cultures were then visualised on an Olympus FV1000 confocal microscope using the 20x lens, 405 laser at settings of 5\%, HV $700 \mathrm{~V}$, Offset $10 \%$ and Gain 1x. The left side of this image shows the fluorescence produced by FSL41 being metabolised in the presence of NfsB_Vv, the right shows the same cells under transmitted light. The activation of FSL41 confirms that this is a stably transfected cell line.

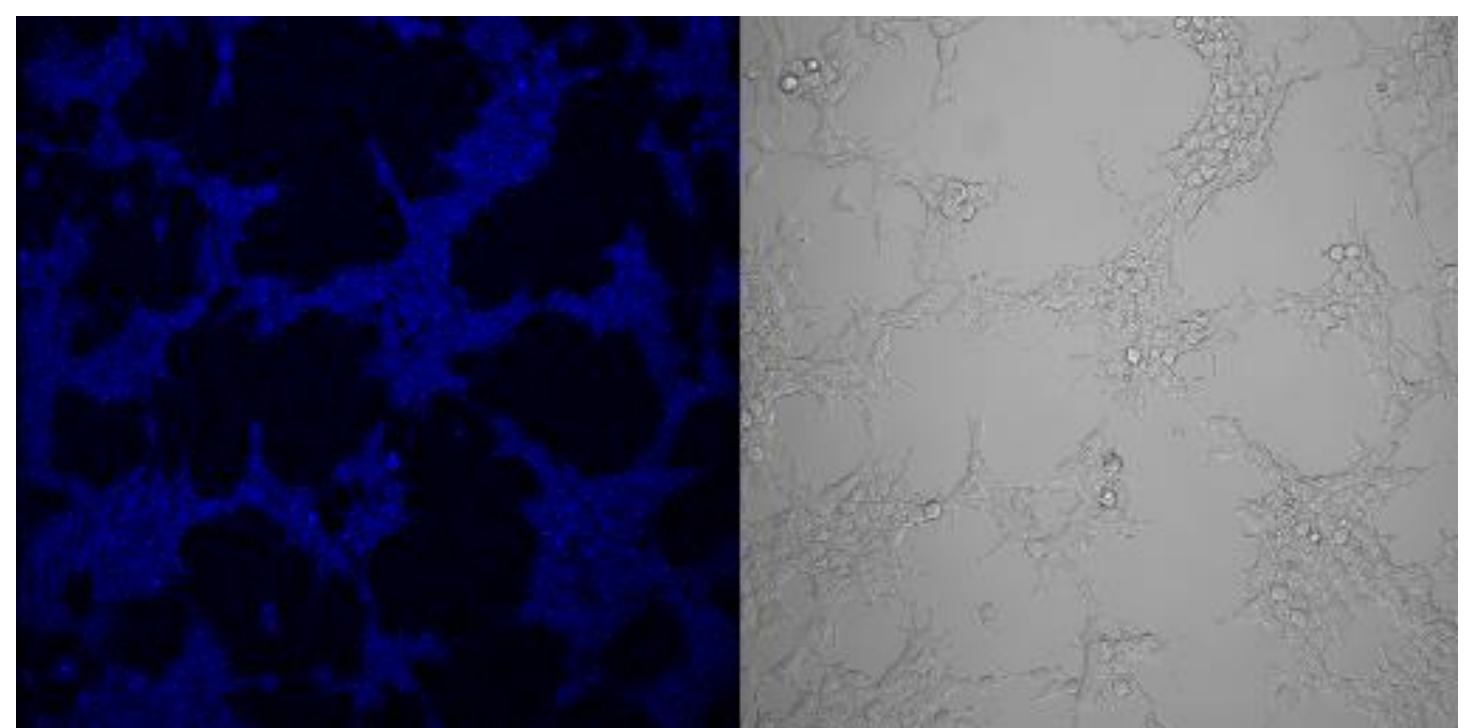

Figure 4.18 HEK293: NfsA_Pp treated with FSL41. Cultures over-expressing nitroreductase NfsA_Pp were treated with $25 \mu \mathrm{M}$ FSL41 and incubated for $1 \mathrm{~h}$. Cultures were then visualised on an Olympus FV1000 confocal microscope using the 20x lens, 405 laser at settings of 5\%, HV $700 \mathrm{~V}$, Offset $10 \%$ and Gain 1x. The left side of this image shows the fluorescence produced by FSL41 being metabolised in the presence of NfsA_Pp, the right shows the same cells under transmitted light. The activation of FSL41 confirms that this is a stably transfected cell line.

HEK293 cells transfected with either NfsA_Pp or NfsB_Vv, demonstrated the same ability to activate masked fluorophores in human cells as they had in bacterial cells. HEK293: NfsA_Pp is capable of activating FSL76 (figure 4.19), but not FSL178 (figure 4.20), while HEK293: NfsB_Vv is capable of activating FSL178 (figure 4.21), but not FSL76 (figure 4.21). In a mixed cell population of HEK293: NfsA_Pp and 
HEK293: NfsB_Vv, distinct cell populations were seen to fluoresce dependent on what nitroreductase they were carrying (figures 4.25 ).

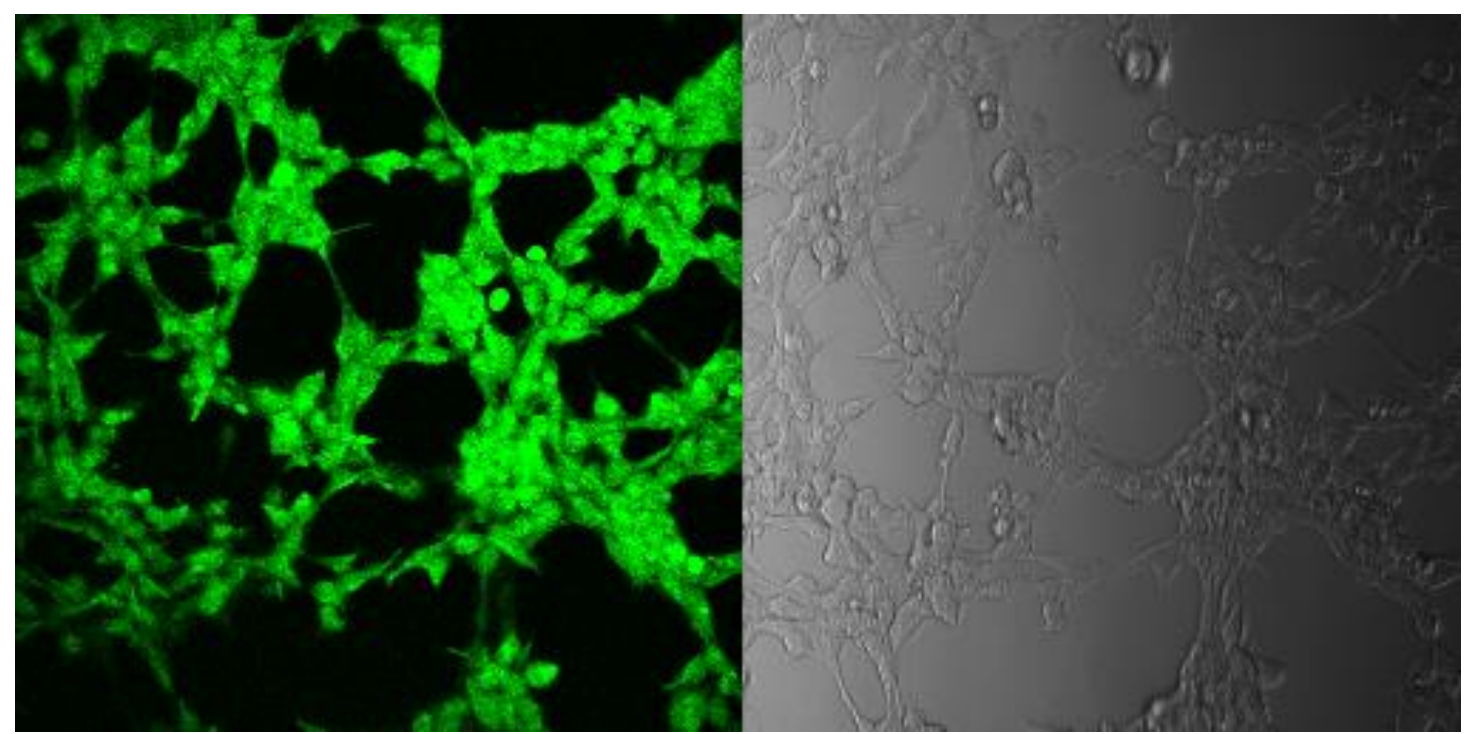

Figure 4.19 HEK293: NfsA_Pp treated with FSL76. Cultures over-expressing nitroreductase NfsA_Pp were treated with $25 \mu \mathrm{M}$ FSL76 and incubated for $1 \mathrm{~h}$. Cultures were then visualised on an Olympus FV1000 confocal microscope using the 20x lens, 488 laser at settings of 5\%, HV $700 \mathrm{~V}$, Offset 10\% and Gain 1x. The left side of this image shows the fluorescence produced by FSL76 being metabolised in the presence of NfsA_Pp, the right shows the same cells under transmitted light.

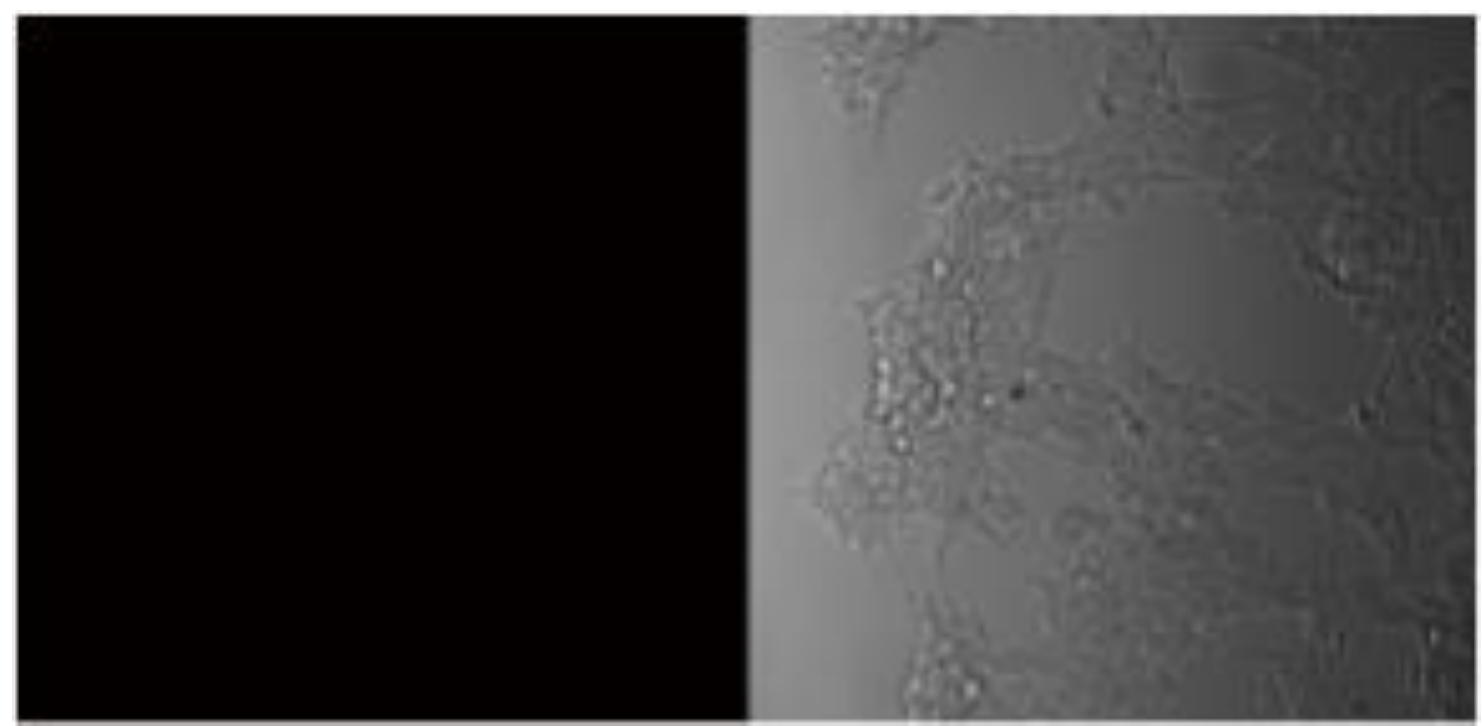

Figure 4.20 HEK293: NfsA_Pp treated with FSL178. Cultures over-expressing nitroreductase NfsA_Pp were treated with $25 \mu \mathrm{M}$ FSL178 and incubated for $1 \mathrm{~h}$. Cultures were then visualised on an Olympus FV1000 confocal microscope using the 20x lens, 647 at settings of laser 5\%, HV 700 V, Offset 10\% and Gain 1x. The left side of this image shows NfsA_Pp is incapable of activating FSL178 as there is no fluorescence observed, the right shows the same cells under transmitted light. 


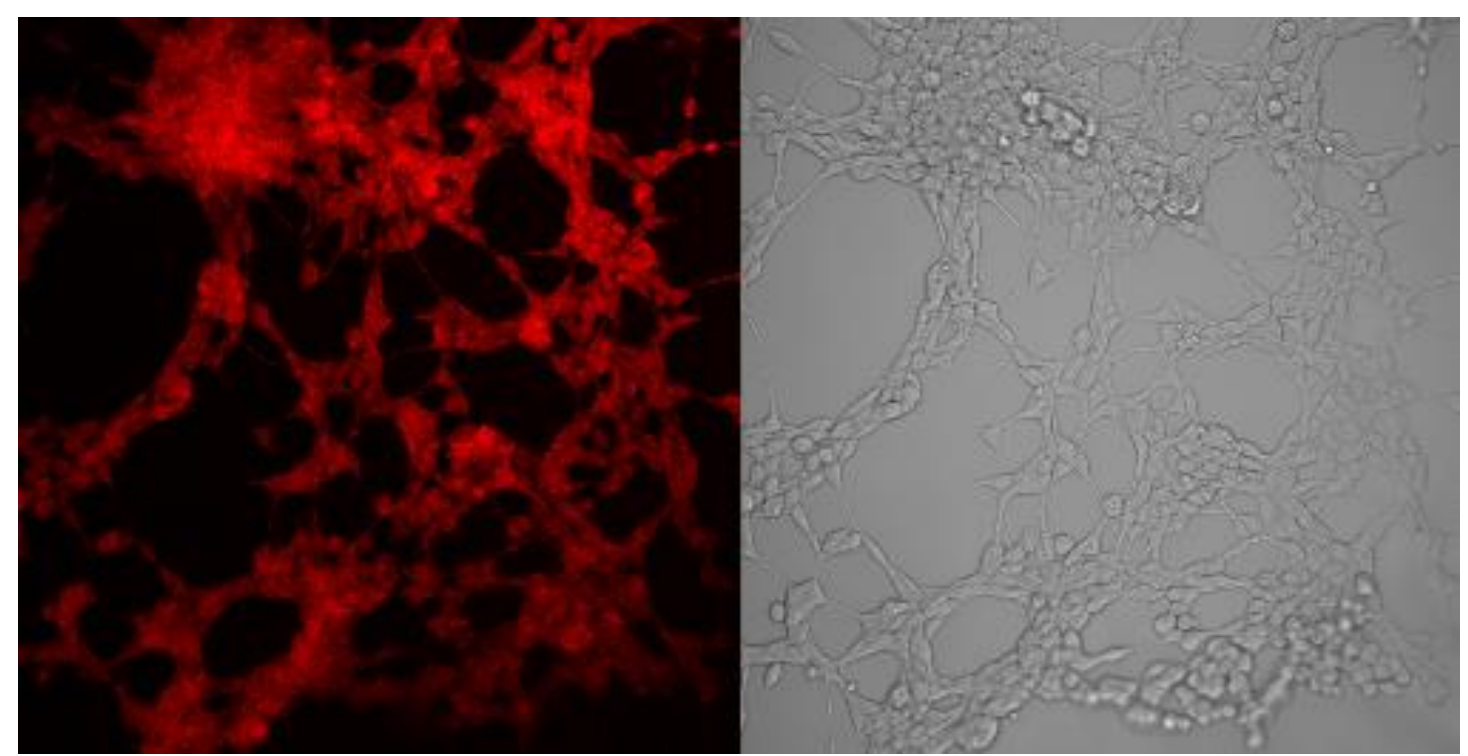

Figure 4.21 HEK293: NfsB_Vv treated with FSL178. Cultures over-expressing nitroreductase NfsB_Vv were treated with $25 \mu \mathrm{M}$ FSL178 and incubated for $1 \mathrm{~h}$. Cultures were then visualised on an Olympus FV1000 confocal microscope using the 20x lens, 647 laser at settings of 5\%, HV $700 \mathrm{~V}$, Offset $10 \%$ and Gain 1x. The left side of this image shows the fluorescence produced by FSL76 being metabolised in the presence of NfsB_Vv, the right shows the same cells under transmitted light.

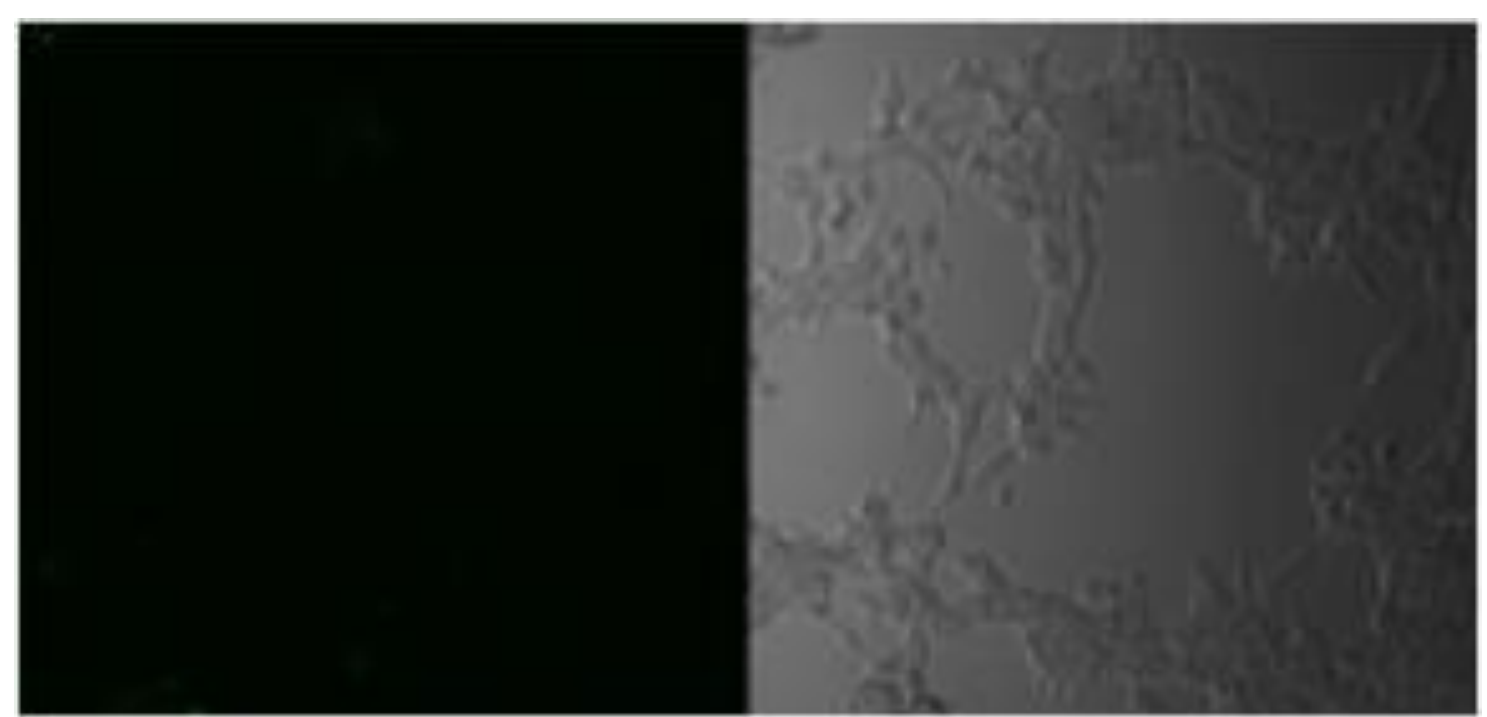

Figure 4.22 HEK293: NfsB_Vv treated with FSL76. Cultures over-expressing nitroreductase NfsB_Vv were treated with $25 \mu \mathrm{M}$ FSL76 and incubated for $1 \mathrm{~h}$. Cultures were then visualised on an Olympus FV1000 confocal microscope using the 20x lens, 488 laser at settings of 5\%, HV $700 \mathrm{~V}$, Offset $10 \%$ and Gain 1x. The left side of this image shows NfsB_Vv is incapable of activating FSL76 as there is no fluorescence observed, the right shows the same cells under transmitted light. 


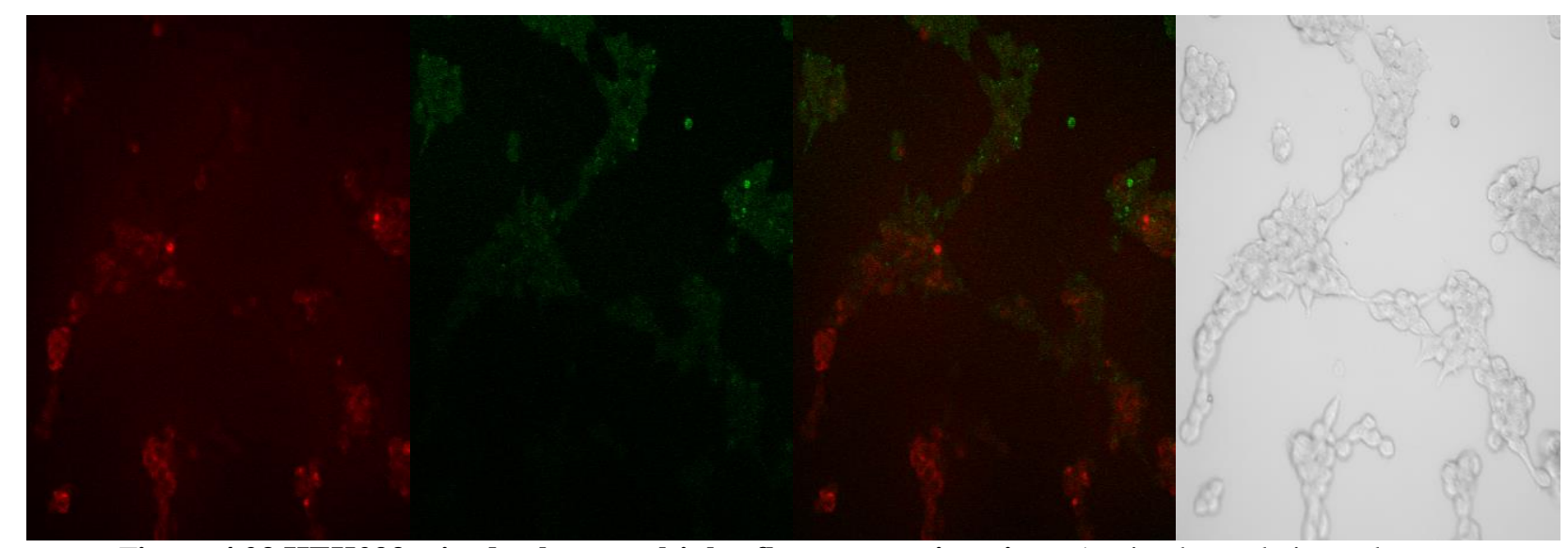

Figure 4.23 HEK293 mixed culture multiplex fluorescence imaging. A mixed population culture containing HEK293: NfsB_Vv and HEK293: NfsA_Pp was treated with both FSL76 and FSL178 and incubated for $1 \mathrm{~h}$. Cultures were then visualised on an Olympus FV1000 confocal microscope using the using the 100x lens, laser 647 at settings of laser 5\%, HV $700 \mathrm{~V}$, Offset $10 \%$ and Gain 1x (far left) 488 laser at settings of 5\%, HV $700 \mathrm{~V}$, Offset $10 \%$ (centre left) and Gain 1x, and The far left image shows distinct areas of FSL178 activation while the centre left image shows distinct areas of FSL76 activation. The centre right image shows these two images superimposed on top of each other, while the far right image shows the same cells under transmitted light.

\subsubsection{Dual fluorescence reporting/ nil bystander antibiotic targeted cell ablation}

Having confirmed that NfsA_Pp and NfsB_Vv exhibited opposing activation profiles with the green probe FSL76 and the red probe FSL178, it was next tested whether these selective fluorescence capabilities could be combined with the selective ablation capabilities demonstrated in chapter 3. This was first evaluated in E. coli $6 \mathrm{KO}$ cells.

Initial experiments undertaken tested the ablation (figures $4.25 ; 4.27$ ) or retention (Figures $4.24 ; 4.26$ ) of fluorescence in single nitroreductase populations challenged with either metronidazole or tinidazole. In a culture over-expressing NfsA_Pp treated with FSL76 and challenged with metronidazole, green fluorescence is clearly visible (figure 4.24). In a culture over-expressing NfsB_Vv treated with FSL178 and challenged with tinidazole, red fluorescence is clearly visible (figure 4.26). However, in a culture over-expressing NfsA_Pp treated with FSL76 and challenged with tinidazole, green fluorescence is not visible, and the cell population is seen to have decreased (figure 4.25). In a culture over-expressing NfsB_Vv treated with FSL178 and challenged with metronidazole, red fluorescence is not visible, and the cell population is seen to have decreased (figure 4.27). These results had been expected from the growth inhibition and fluorescence screening (figure 4.10). 


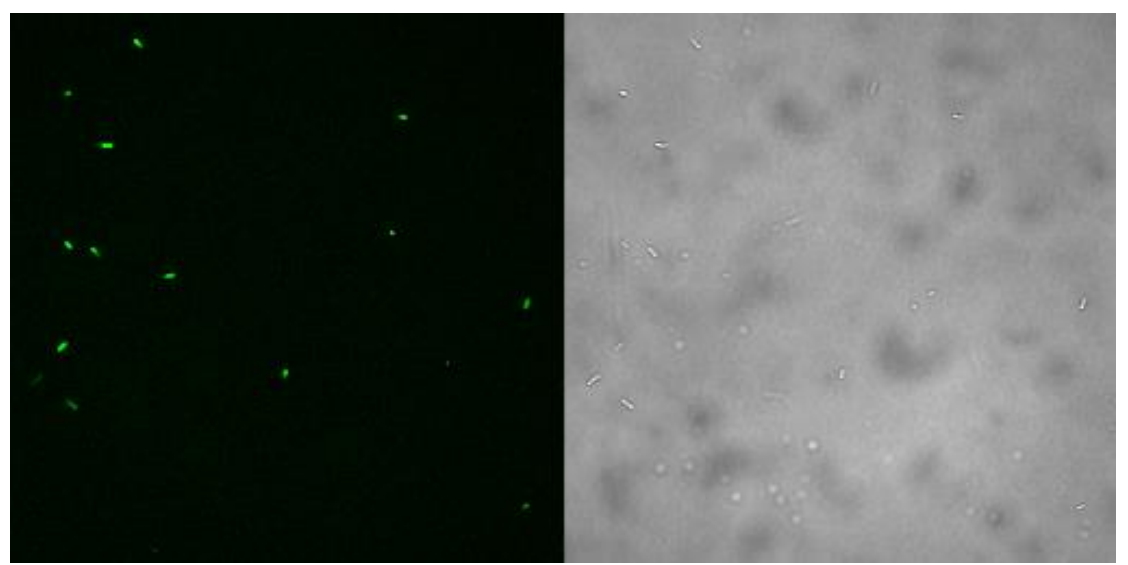

Figure 4.24 6KO E. coli over-expressing NfsA_Pp treated with FSL76 and challenged with metronidazole. Cultures over-expressing nitroreductase NfsA_Pp were treated with $25 \mu \mathrm{M}$ FSL76 and $50 \mu \mathrm{M}$ metronidazole and incubated for $4 \mathrm{~h}$. Cultures were fixed to slides using $4 \%$ paraformaldehyde then imaged on an Olympus FV1000 confocal microscope using the 100x lens, 488 laser at settings of 5\%, HV $700 \mathrm{~V}$, Offset $10 \%$ and Gain 1x. NfsA_Pp activates FSL76 to produce green fluorescence (left panel), because the cells over-expressing this nitroreductase are unaffected by metronidazole. The right shows the same bacteria under transmitted light.

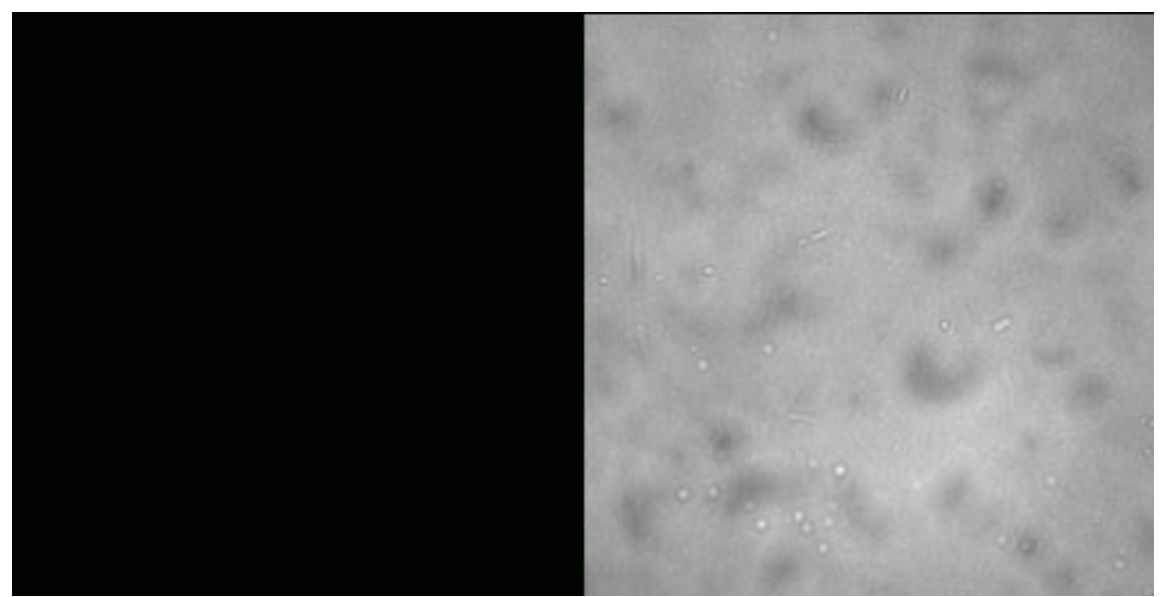

Figure 4.25 6KO E. coli over-expressing NfsA_Pp treated with FSL76 and challenged with tinidazole. Cultures over-expressing nitroreductase NfsA_Pp were treated with $25 \mu \mathrm{M}$ FSL76 and 50 $\mu \mathrm{M}$ tinidazole and incubated for $4 \mathrm{~h}$. Cultures were fixed to slides using $4 \%$ paraformaldehyde then imaged on an Olympus FV1000 confocal microscope using the 100x lens, 488 laser at settings of 5\%, HV 700 V, Offset $10 \%$ and Gain 1x. Although NfsA_Pp activates FSL76, green fluorescence is not visible, shown on the left, as the nitroreductase also activates tinidazole which ablates the cell population as can be seen on the right.

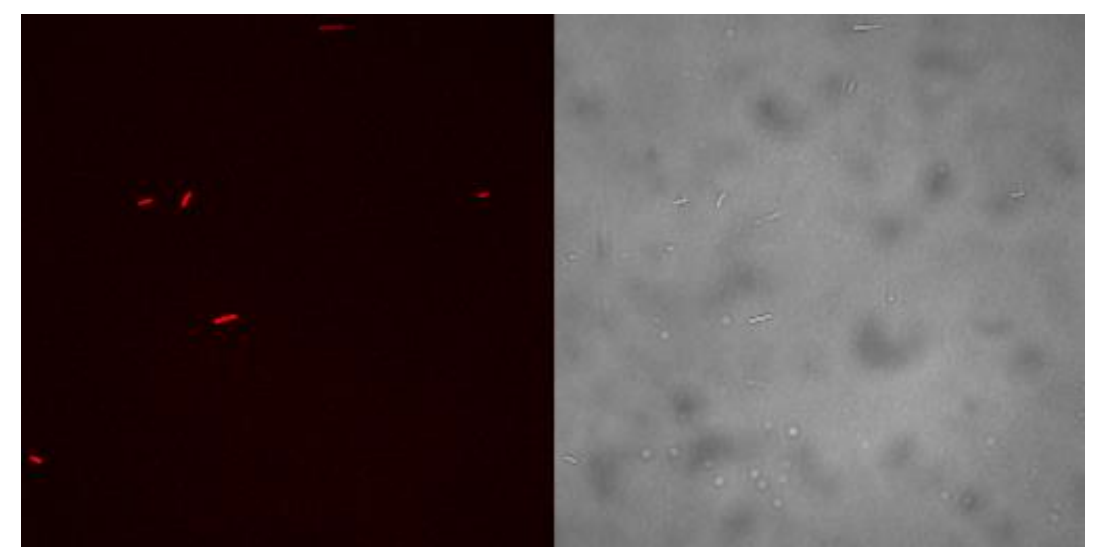

Figure 4.26 6KO $E$. coli over-expressing NfsB_Vv treated with FSL178 and challenged with tinidazole. Cultures over-expressing nitroreductase NfsB_Vv were treated with $25 \mu \mathrm{M}$ FSL178 and 
challenged with $75 \mu \mathrm{M}$ tinidazole and incubated for $4 \mathrm{~h}$. Cultures were fixed to slides using $4 \%$ paraformaldehyde then imaged on an Olympus FV1000 confocal microscope using the 100x lens, 647 laser at settings of 5\%, HV $700 \mathrm{~V}$, Offset $10 \%$ and Gain 1x. NfsB_Vv activates FSL178 to produce red fluorescence. As $\mathrm{NfsB}_{-} \mathrm{Vv}$ is incapable of activating tinidazole, the fluorescence is still visible in its presence. This can be seen on the left. The right hand shows the same bacteria under transmitted light.

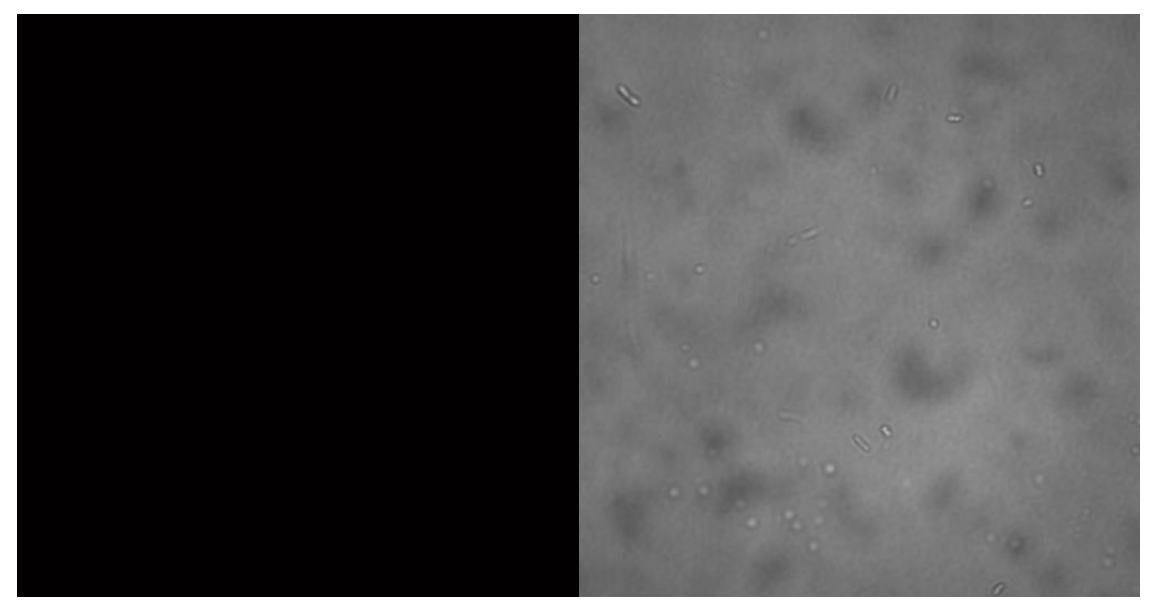

Figure 4.27 6KO $E$. coli over-expressing NfsB_Vv treated with FSL178 and challenged with metronidazole. Cultures over-expressing nitroreductase NfsB_Vv were treated with $25 \mu \mathrm{M}$ FSL178 and challenged with $50 \mu \mathrm{M}$ metronidazole and incubated for $4 \mathrm{~h}$. Cultures were fixed to slides using 4\% paraformaldehyde then imaged on an Olympus FV1000 confocal microscope using the 100x lens, 647 laser at settings of 5\%, HV 700 V, Offset 10\% and Gain 1x. Although NfsB_Vv activates FSL178, red fluorescence is not visible, shown on the left, as the nitroreductase also activates metronidazole which ablates the cell population as can be seen on the right.

As dual opposing fluorophore and antibiotic activation profiles had been demonstrated for NfsA_Pp and NfsB_Vv, replicate cultures of cells expressing either nitroreductase were mixed, then treated with both fluorophores (FSL76 and FSL178) together, followed by challenge with either $75 \mu \mathrm{M}$ tinidazole or $50 \mu \mathrm{M}$ metronidazole. In mixed cell populations the masked fluorophores and nil bystander antibiotics behaved as had been expected as well, with the presence of both NfsA_Pp and NfsB_Vv and FSL76 and FSL178 challenged with metronidazole showing only green fluorescence, as the red fluorescing population also activates metronidazole therefore eliminating the cell population (figure 4.31). The opposite was seen in the same mixed population of both NfsA_Pp and NfsB Vv and FSL76 and FSL178 when challenged with tinidazole, showing only red fluorescence, as the green fluorescing population also activates tinidazole therefore eliminating the cell population (figure 4.32). 


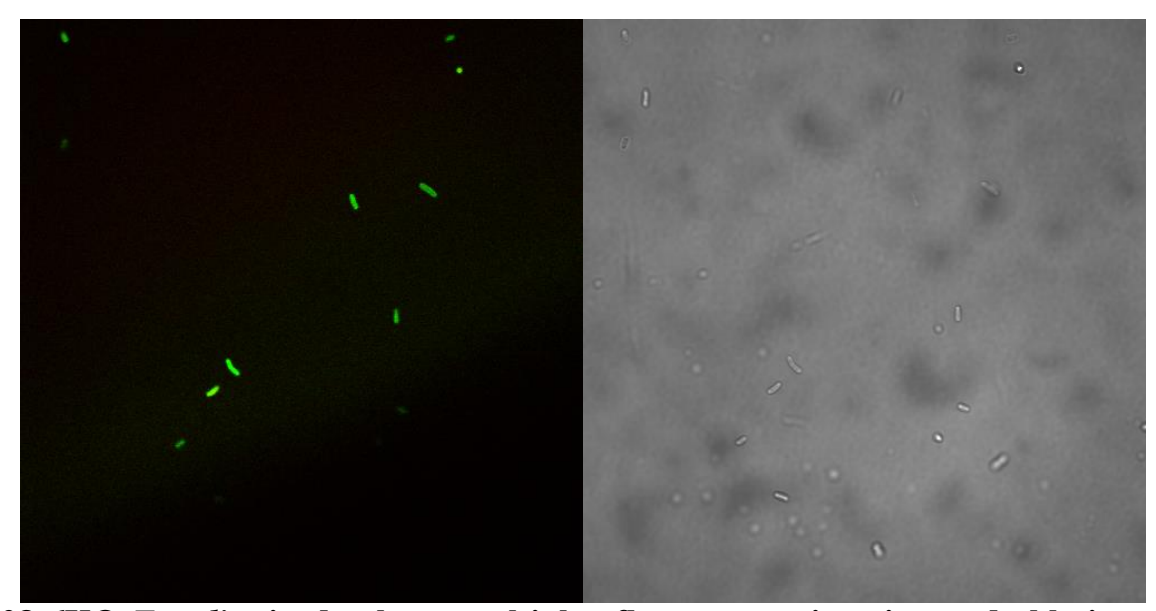

Figure 4.28 6KO $E$. coli mixed culture multiplex fluorescence imaging and ablation challenged with metronidazole. A mixed population culture containing $6 \mathrm{KO}$ E. coli over-expressing NfsB_Vv and NfsA_Pp was treated with both FSL76 $(25 \mu \mathrm{M})$ and FSL178 $(25 \mu \mathrm{M})$ and metronidazole $(50 \mu \mathrm{M})$ and incubated for $4 \mathrm{~h}$. Cultures were fixed to slides using $4 \%$ paraformaldehyde then imaged on an Olympus FV1000 confocal microscope using the 100x lens, 488 laser at settings of 5\%, HV $700 \mathrm{~V}$, Offset $10 \%$ and Gain 1x, and 647 laser at settings of 5\%, HV $700 \mathrm{~V}$, Offset 10\% and Gain 1x. Although NfsB_Vv activates FSL178 to produce red fluorescence, none can be seen as the bacterial population has been ablated due to the ability of $\mathrm{NfsB}_{-} \mathrm{Vv}$ to activate metronidazole. NfsA_Pp activates FSL76 to produce green fluorescence, as it is incapable of activating metronidazole, the green fluorescence is still visible in its presence as the NfsA_Pp bacterial population has not been ablated. This can be seen on the left. The right hand shows the same bacteria under transmitted light.

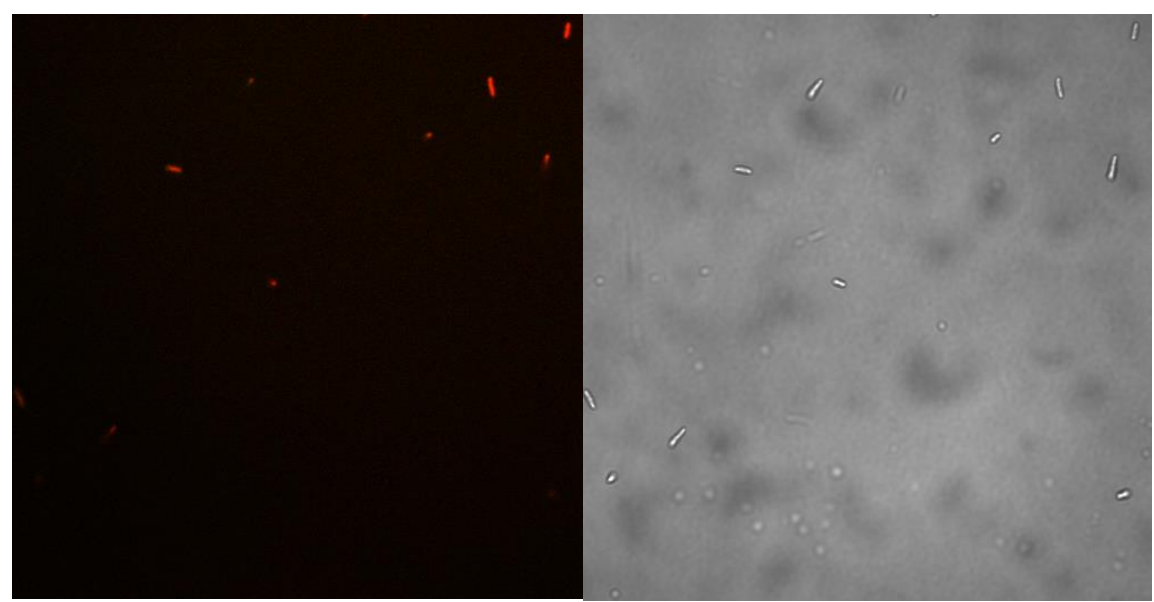

Figure 4.29 6KO $E$. coli mixed culture multiplex fluorescence imaging and ablation challenged with tinidazole. A mixed population culture containing 6KO E . coli over-expressing NfsB_Vv and NfsA_Pp was treated with both FSL76 and FSL178 and tinidazole and incubated for $\overline{4} \mathrm{~h}$. Cultures were fixed to slides using $4 \%$ paraformaldehyde then imaged on an Olympus FV1000 confocal microscope using the 100x lens, 488 laser at settings of 5\%, HV $700 \mathrm{~V}$, Offset $10 \%$ and Gain 1x, and 647 laser at settings of 5\%, HV 700 V, Offset 10\% and Gain 1x. Although NfsA_Pp activates FSL76 to produce green fluorescence, none can be seen as the bacterial population has been ablated due to the ability of NfsA_Pp to activate tinidazole. NfsB_Vv activates FSL178 to produce red fluorescence, as it is incapable of activating tinidazole, the red fluorescence is still visible in its presence as the NfsB_Vv bacterial population has not been ablated. This can be seen on the left. The right hand shows the same bacteria under transmitted light.

Finally, when cultures of E. coli strains over-expressing either NfsA_Pp or NfsB_Vv were mixed and then challenged with both $75 \mu \mathrm{M}$ tinidazole and $50 \mu \mathrm{M}$ metronidazole, complete cell elimination and disappearance of any fluorescence was observed (figure 4.30). 


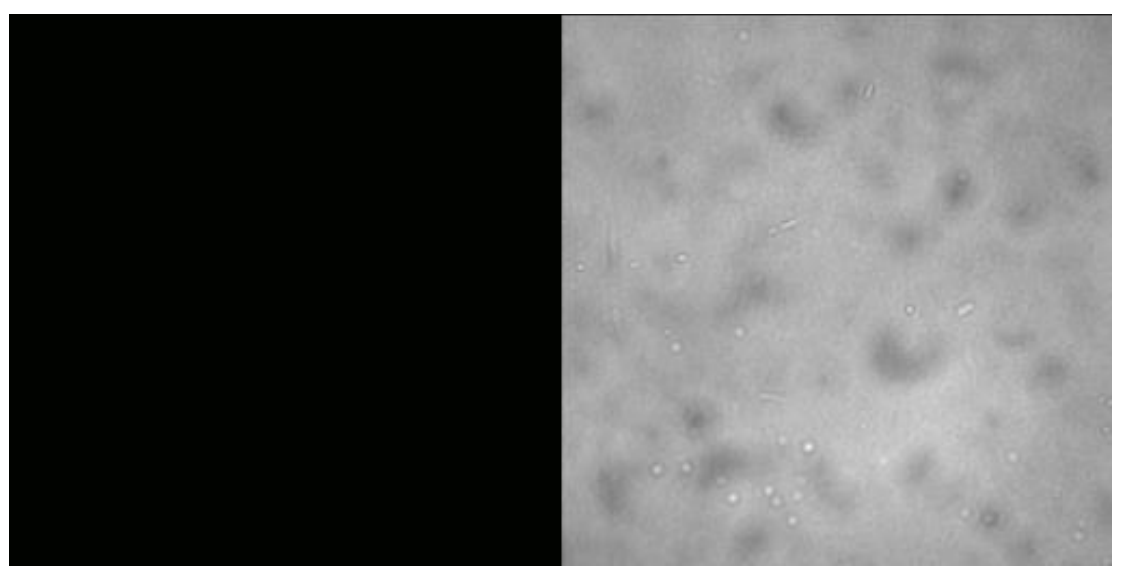

Figure 4.30 6KO $E$. coli mixed culture multiplex fluorescence imaging challenged with metronidazole and tinidazole. A mixed population culture containing $6 \mathrm{KO}$ E. coli over-expressing NfsB_Vv and NfsA_Pp was treated with both FSL76 and FSL178 and metronidazole $50 \mu \mathrm{M}$ and tinidazole $75 \mu \mathrm{M}$ and incubated for $4 \mathrm{~h}$. Cultures were fixed to slides using $4 \%$ paraformaldehyde then imaged on an Olympus FV1000 confocal microscope using the 100x lens, 488 laser at settings of 5\%, HV $700 \mathrm{~V}$, Offset 10\% and Gain 1x, and 647 laser at settings of 5\%, HV 700 V, Offset 10\% and Gain 1x. Although NfsA_Pp activates FSL76 to produce green fluorescence, and NfsB_Vv activates FSL178 to produce red fluorescence, neither can be seen as both bacterial populations have been ablated due to the ability of NfsA_Pp to activate tinidazole and the ability of NfB_VV to activate metronidazole. This can be seen on the left. The right images the same area under transmitted light, and confirms the bacterial population has been ablated.

The dual fluorescence reporter/ cell ablation system showed the same effect in human cells as in bacterial cells. The addition of $150 \mu \mathrm{M}$ tinidazole to HEK293: NfsA_Pp cells treated with FSL76 showed ablation of the cell population, and almost complete disappearance of fluorescence (figure 4.31), while the addition of $150 \mu \mathrm{M}$ metronidazole to HEK293: NfsB_Vv cells treated with FSL178 showed ablation of the cell population, and almost complete disappearance of fluorescence (figure 4.32). 


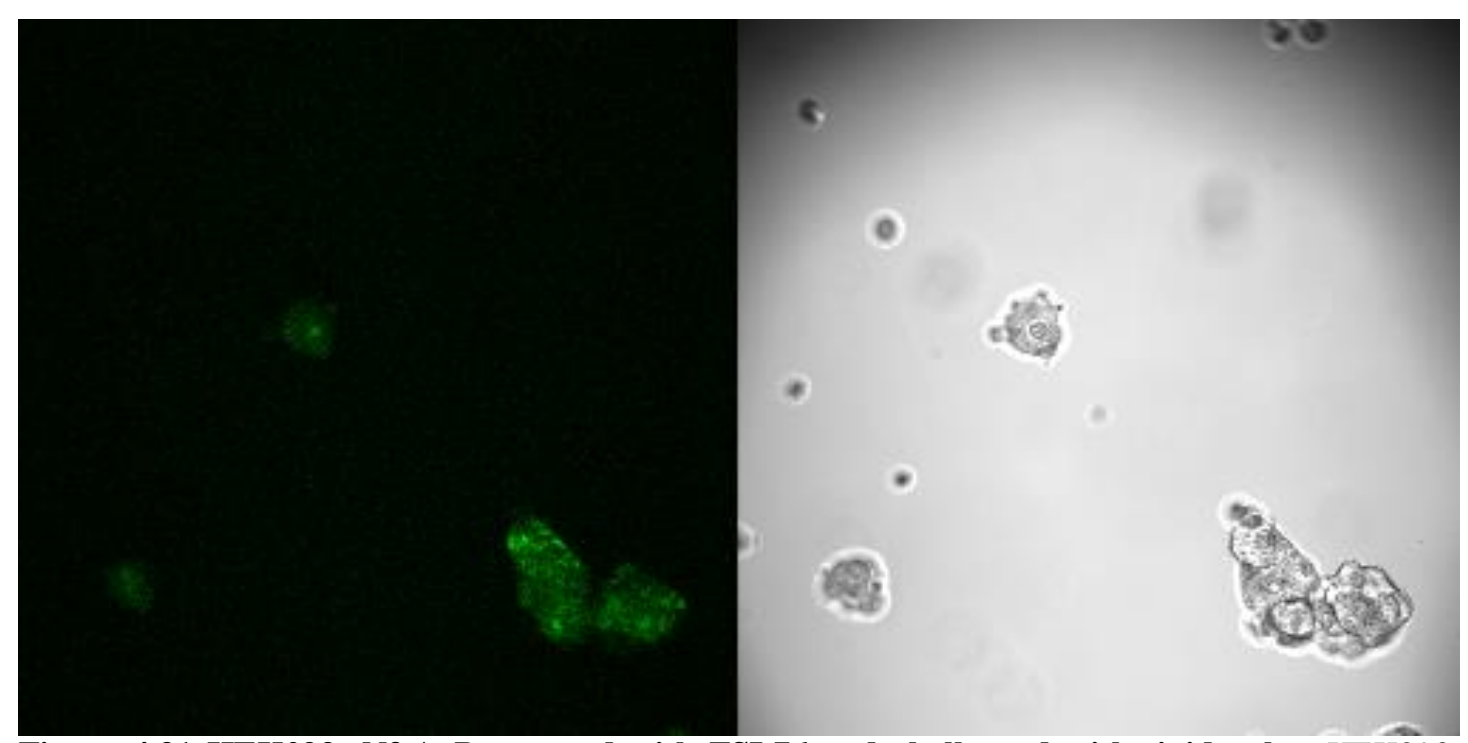

Figure 4.31 HEK293: NfsA_Pp treated with FSL76 and challenged with tinidazole. HEK293: NfsA_Pp cultures were treated with $25 \mu \mathrm{M}$ FSL76 and challenged with $150 \mu \mathrm{M}$ tinidazole and incubated for $1 \mathrm{~h}$. Cultures were then imaged on an Olympus FV1000 confocal microscope using the 20x lens, 488 laser at settings of 5\%, HV 700 V, Offset 10\% and Gain 1x. NfsA_Pp activates FSL76 to produce fluorescence, and tinidazole which ablates the cell population. As can be seen under transmitted light on the right hand side, the cell morphology has deteriorated, and the cell population has been ablated. The left side shows that there is still a small amount of green fluorescence visible, implying that full cell death has not occurred.

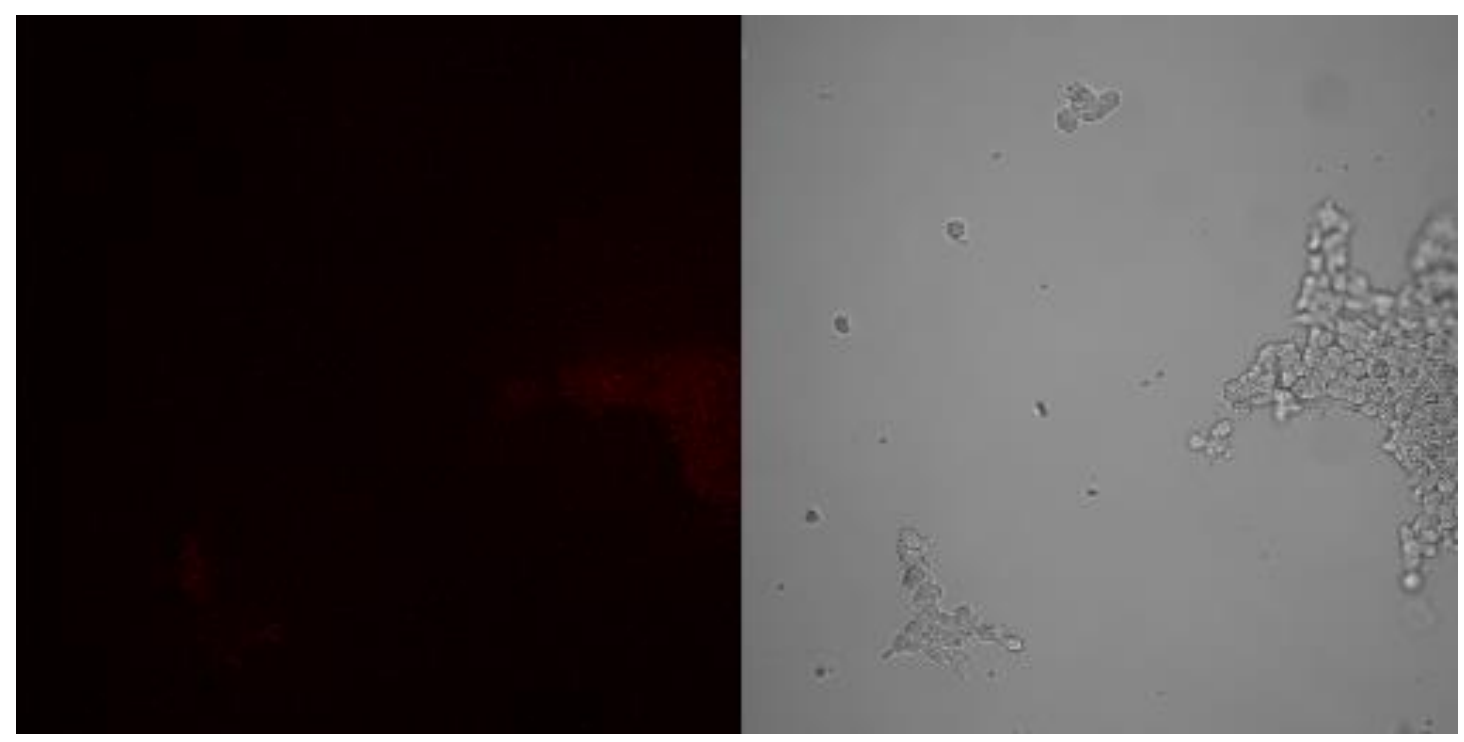

Figure 4.32 HEK293: NfsB_Vv treated with FSL178 and challenged with metronidazole.

HEK293: NfsB_Vv cultures were treated with $25 \mu \mathrm{M}$ FSL178 and challenged with $150 \mu \mathrm{M}$ metronidazole and incubated for $1 \mathrm{~h}$. Cultures were then imaged on an Olympus FV1000 confocal microscope using the 20x lens, 647 laser at settings of 5\%, HV $700 \mathrm{~V}$, Offset $10 \%$ and Gain 1x. NfsB_Vv activates FSL178 to produce red fluorescence, and metronidazole which ablates the cell population. As can be seen under transmitted light on the right hand side, the cell morphology has deteriorated, and the cell population has been ablated. The left side shows that there is little to no fluorescence visible, confirming near-complete cell death.

Results from bacterial cells were again mirrored in human cell lines in mixed culture populations. When a co-culture of HEK293: NfsA_Pp and HEK293: NfsB_Vv was 
incubated with both FSL76 and FSL178, and challenged with $50 \mu \mathrm{M}$ metronidazole, only green fluorescence was observed. This is because the red fluorescing HEK293: NfsB_Vv population also activates metronidazole, leading to its clean ablation from the co-culture (Figure 4.33). The opposite phenotype was seen in a replicate coculture incubated with both masked fluorophores and challenged with $75 \mu \mathrm{M}$ tinidazole; only red fluorescence was observed, as the green fluorescing HEK293:

NfsA_Pp population also activates tinidazole, leading to ablation (Figure 4.34).

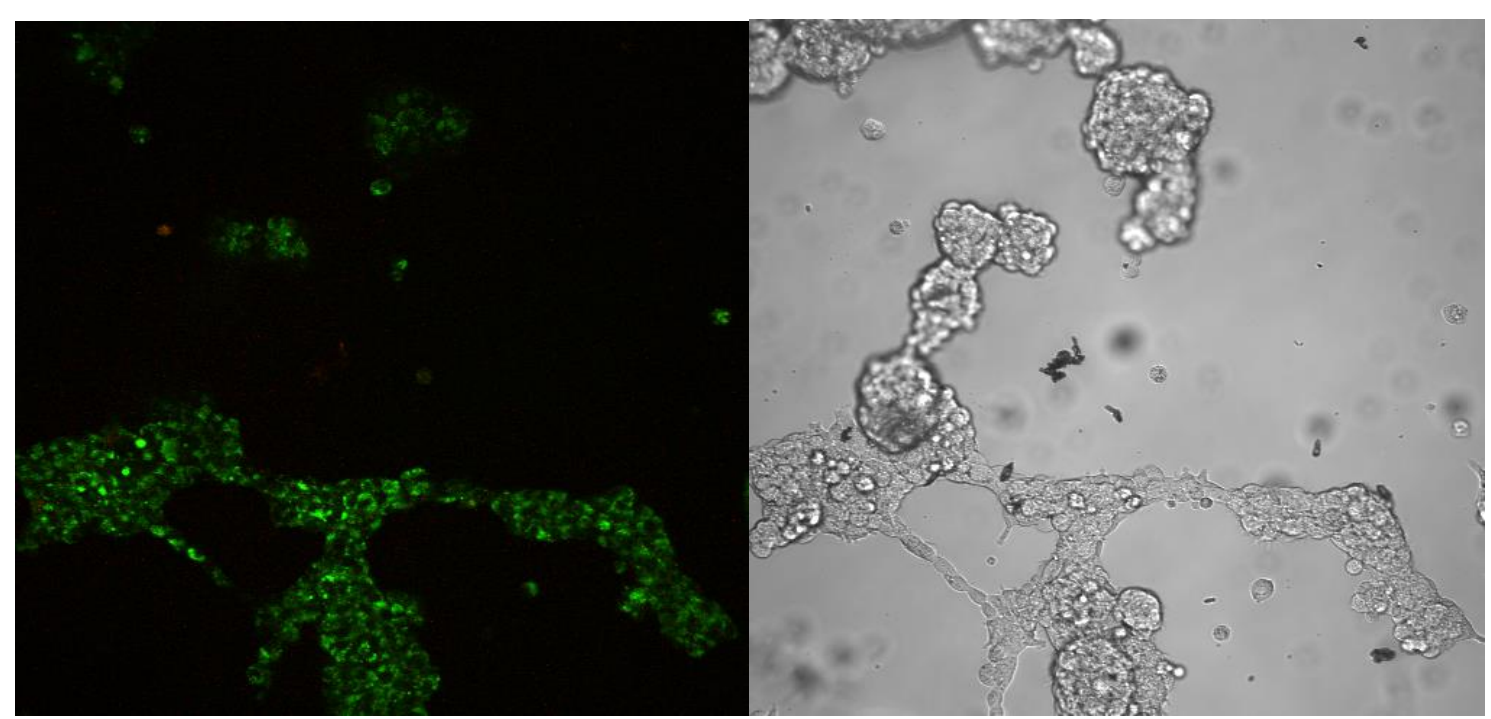

Figure 4.33 HEK293 mixed culture multiplex fluorescence imaging and ablation challenged with metronidazole. A mixed population culture containing HEK293: NfsB_Vv and HEK293: NfsA_Pp was treated with both FSL76 $(25 \mu \mathrm{M})$ and FSL178 $(25 \mu \mathrm{M})$ and metronidazole $(150 \mu \mathrm{M})$ and incubated for $1 \mathrm{~h}$. Cultures were then imaged on an Olympus FV1000 confocal microscope using the 100x lens, 488 laser at settings of 5\%, HV $700 \mathrm{~V}$, Offset $10 \%$ and Gain 1x, and 647 laser at settings of 5\%, HV 700 V, Offset 10\% and Gain 1x. Although NfsB_Vv activates FSL178 to produce red fluorescence, none can be seen as the cell population has been ablated due to the ability of NfsB_Vv to co-activate metronidazole, NfsA_Pp activates FSL76 to produce green fluorescence, and as it is incapable of coactivating metronidazole, the green fluorescence is still visible. This can be seen on the left. The right hand shows the same area under transmitted light. 


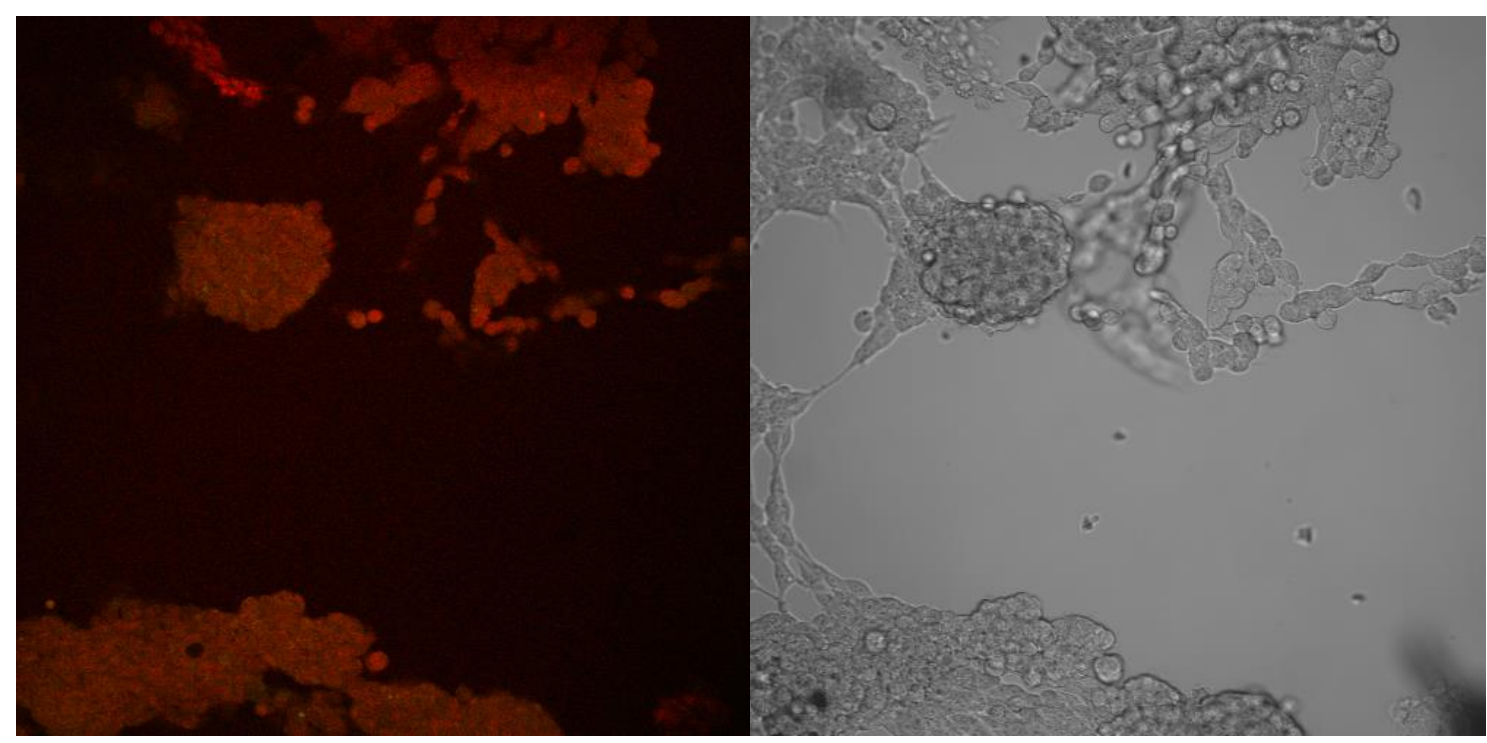

Figure 4.34 HEK293 mixed culture multiplex fluorescence imaging and ablation challenged with tinidazole. A mixed population culture containing HEK23: NfsB_Vv and HEK293: NfsA_Pp was treated with both FSL76 and FSL178 and tinidazole and incubated for $4 \mathrm{~h}$. Cultures were then imaged on an Olympus FV1000 confocal microscope using the 100x lens, 488 laser at settings of 5\%, HV 700 V, Offset 10\% and Gain 1x, and 647 laser at settings of 5\%, HV 700 V, Offset 10\% and Gain 1x. Although NfsA_Pp activates FSL76 to produce green fluorescence, none can be seen as the cell population has been ablated due to the ability of NfsA_Pp to co-activate tinidazole. NfsB_Vv activates FSL178 to produce red fluorescence, as it is incapable of activating tinidazole, the red fluorescence is still visible. This can be seen on the left. The right hand shows the same area under transmitted light.

\subsection{Discussion}

The previous fluorescence screening work by Dr Horvat made the choice of masked fluorophores to be rescreened in the $6 \mathrm{KO}$ library easy to narrow down. The selections were based on masked fluorophores that had previously shown high activity with nitroreductases chosen for their selective prodrug and fluorophore activation profiles. Dr Horvat's work also helped establish FSL41 as a general indicator of nitroreductase activity; this enabled the initial optimisation experiments with alternative fluorescent probes (figure 4.9-4.14) to be run with confidence, and allowed for the functional validation of successful transfection; with the presence of fluorescence in the human cell line populations providing proof that the nitroreductase is present and active (figures $4.16 ; 4.17 ; 4.18$ ).

Although targeted cell ablation systems using fluorescent proteins as reporter systems are in current use in the field of developmental biology, there would be advantages in reporting directly on the nitroreductase as opposed to a bicistronic fluorescent reporter 
protein. Bicistronic systems have been shown to have cause delayed replication time of transfected cells, and to produce less protein than monocistronic systems (Schaller et al., 2007). These disadvantages could be overcome by the use of masked fluorophores, thereby negating the need for the use of a bicistronic system for reporting on targeted cell ablation.

These initial optimisation protocols enabled an investigation into the ablation of fluorescence for developmental biology, first utilising the nitroreductase which had been identified as having the most efficacy with metronidazole in chapter 3, NfsB_Vh. The results of this study demonstrate that when bacteria over-expressing NfsB_Vh are treated only with FSL41, there is a high level of fluorescence, and the growth of the cell population appears to remain unaltered (figure 4.4). When a cell population containing NfsB_Vh treated with FSL41 was challenged with metronidazole, however, the fluorescence levels and the corresponding cell density levels also decreased (figure 4.5), confirming that the addition of a nil bystander antibiotic was responsible for the decline in cell population, and consequently the disappearance of fluorescence. These results were mirrored in experiments carried out in transfected HCT-116 human cell lines (figures 4.6; 4.7). This was important as it has been previously shown that not all nitroreductases behave the same in human cells as bacterial cells, and this technology is aimed to be used within the field of developmental biology in eukaryotic cells.

With the basic dual imaging and ablation tools validated it was next sought to determine whether these could be expanded into a multiplex system of nil bystander antibiotics and masked fluorophores, which might provide dual ablation/ reporter capabilities in a mixed cell population. Results from chapter 3, and from the fluorescence screening indicated that the two most promising nitroreductases for this were NfsA_Li, and NfsB_Vv. As has been stated, NfsA_Pp was used for these experiments in the place of NfsA_Li, as NfsA_Li was unable to be taken through to human cell line stage due to technical difficulties and time constraints. NfsA_Pp is capable of activating the nil bystander antibiotic tinidazole and the masked fluorophore FSL76, which NfsB_Vv is not, and exhibits at most low-level activity with the nil bystander antibiotic metronidazole and the masked fluorophore FSL178, 
which NfsB_Vv does activate. These capabilities were found to translate consistently from individual bacterial (figures 4.9-4.13) to human (figures 4.19-4.22) cell cultures.

The next step to validate this dual reporter/ ablation system was to test these techniques in mixed culture populations. In both bacterial cells, and human cell lines distinct populations in mixed cultures are observed either enabling the fluorescence of FSL76 or FSL 178 (figures 4.13; 4.25). Upon the addition of tinidazole to these mixed cultures, the population of green fluorescing cells disappeared while the red fluorescing cells remained (figures 4.29, 4.34), as would be expected given that NfsA_Vv is resistant to tinidazole but can activate FSL178. In contrast, upon the addition of metronidazole to the mixed cultures, the population of red fluorescing cells disappeared while the green fluorescing cells remained (Figures 4.28, 4.33). Again, this is what would be expected as NfsB_Pp is resistant to metronidazole, but can activate FSL76. This result confirms the ability of initial testing of nitroreductase systems to be undertaken in bacterial systems, and provides strong evidence that a multiplexed dual reporter/ ablation system should be possible in vivo.

Thus, these results give a comprehensive proof of principle that the combination of masked fluorophores and nil bystander antibiotics used in conjunction with nitroreductases with opposing activation profiles have the potential to be utilised in developmental and regenerative biology studies for the purpose of a multiplexed dual reporter/ ablation system. Developmental and regenerative biology studies have already employed the use of a single nitroreductase (E. coli NfsB_Ec), exploiting its nitro-reducing capabilities for targeted cell ablation in individual cell types using built in fluorescent proteins as reporters (White and Mumm, 2013). The expanded approach presented in this chapter, however, could enable a temporal and spatial reporter/ ablation system targeted to two different cell types in the same organism, with increased sensitivity and flexibility. 


\section{Summary, Conclusions, and Future Directions}

\subsection{Research motivation}

\subsubsection{GDEPT}

The ability of nitroreductases to reduce a range of nitroquenched compounds has offered potential for an all-encompassing GDEPT system, whereby a single nitroreductase is able to activate a PET imaging agent to confirm localisation of the vector carrying the therapeutic gene, a prodrug for the treatment of cancer, and a nil bystander antibiotic as a safety mechanism to eliminate of the delivery vector.

GDEPT is a fast developing and promising field of research into cancer treatment, the development of which is being boosted by clinical advances and mainstream acceptance of next-generation tumour-targeting viral vectors (Schmidt, C. 2011) The discovery of nitroreductases and their ability to reduce nitroquenched compounds has been exploited to activate numerous clinically advanced prodrug combinations that were originally developed to be reduced by hypoxic regions of cancerous tumours (Brown, 2007; Patterson et al., 2007, Denny, 2003; Singleton et al., 2007). The imaging of the distribution and magnitude of the therapeutic gene expression is an important component of GDEPT; it enables visualisation of the vector to ensure ithas localised to the tumour and is viable throughout the treatment process. The development of fluorescent imaging methods to detect the gene expression could enable this to be a possibility, providing information on the optimal time and duration for prodrug administration (Singleton et al., 2007). Another highly valuable use of this technology would be to monitor real time expression of therapeutic gene expression in the presence of a prodrug throughout therapy, trumping current methodology which focuses on the size of the tumour post treatment to evaluate drug efficacy. Nitroreductases that are capable of activating masked fluorophores are a promising tool, as there are currently only a small number of reporter systems that can be used in a non-invasive manner to directly report on enzyme activity. Importantly, this approach removes the need to introduce a secondary reporter gene to the system.

Another important step in the development of a GDEPT system is the need to eliminate the vector used to deliver the therapeutic gene, post therapy. This is 
extremely important to qualify for FDA approval, as many of the vectors used are either viruses or bacteria of pathogenic origin due to the efficiency from their natural life cycle as viruses, are capable of effectively invading human cells and expressing their genes within the cell. For elimination of viral vectors in particular, it is likely to be essential that the ablating nitroreductase is expressed early in the life cycle, and that it is highly active at converting a nil-bystander antibiotic into a potent cytotoxin. The identification in this thesis of several nitroreductases that are far more active than E. coli $\mathrm{NfsB}$ with metronidazole, as well as several new and promising nitroreductase/antibiotic combinations, will provide fertile ground for further development of systems to efficiently eliminate labelled viruses from human cells, either in vitro or in vivo.

The addition of imaging agents to confirm the localisation of the vector containing the therapeutic gene and at which point there is the highest gene expression in these cells but not surrounding healthy cells, combined with a mechanism to void the system of the vector post treatment, could greatly advance the development of GDEPT as a viable tool for the treatment of a wide range of solid tumour cancers.

\subsubsection{Novel developmental biology tools}

The ability of nitroreductases to activate nil bystander antibiotics that ablate only the target cells where the nitroreductase is present, leaving surrounding cells untouched, has also been exploited in the fields of developmental and regenerative biology.

These fields exploit the ability of nitroreductases to act as suicide genes that can ablate a specific cell population. In the case of developmental biology, these capabilities can help identify key functions the target cells have in the developing organism, and what diseases may result when an organism lacks the target cell type. In regenerative studies, the ability to ablate a specific cell population can enable inferences as to whether that cell population is capable of regenerating, and what biological factors are essential for this to take place. The added bonus of having the nitroreductase system in place instead of a gene knockout, is that the specific cell type that is targeted can be ablated at any time during development; therefore enabling the study into the effect of ablation at different time points during development rather 
than just one.

A handful of contemporary studies have exploited the ability of nitroreductases to reduce nil bystander antibiotics for targeted ablation of a specific cell population. To do this, a fluorescent reporter and a nitroreductase, NfsB_Ec, are fused to a promoter of interest. Upon the addition of the nil bystander antibiotic metronidazole, the cell population containing the nitroreductase fusion molecule are ablated, and surrounding cells are left untouched. Transparent organisms such as Xenopus and zebrafish are ideal models for reporting on the ablation via a fluorescent fusion protein.

The Ackerley lab's access to nitro-quenched masked fluorophores as well as numerous nitro-quenched nil bystander antibiotics, has enabled us to propose advancing on existing capabilities via integration of only a nitroreductase in place of the gene of interest; i.e. no accompanying fluorescent protein, these being functionally replaced by the masked fluorophore substrates. This would have the same visual effect as the GFP/ nitroreductase system, with the absence of fluorescence after the addition of the nil bystander antibiotics indicating ablation of the cells expressing the nitroreductase protein.

The ability to report on the cell population using fluorescent imaging is important, as it allows the area to be ablated to be visualised prior to addition of the nil bystander antibiotic, ensuring that expression of the nitroreductase gene is exclusive to the target tissue. It also enables the efficacy of ablation to be evaluated directly, post-addition of the nil bystander antibiotic.

Expanding on the basic scenario, we have proposed a two way reporter/ ablation system; wherein one nitroreductase is active with a specific masked fluorophore and nil bystander antibiotic, and inactive with a different masked fluorophore and nil bystander antibiotic combination, while a second nitroreductase exhibits the opposing activation profile, being inactive with the first masked fluorophore/ nil bystander combination, and active with the other.

This system would allow the study of multiple cell types at once, with the ability to study the effect of ablating two different cell types at specific times during 
development; either simultaneously or subsequently. Although complete ablation of the target cell population is not currently achievable, the identification in this thesis of nitroreductases with superior metronidazole activation to $E$. coli $\mathrm{NfsB}$, as well as other promising nitroreductase/ nil bystander antibiotic combinations may make this goal a reality.

\subsection{Key Findings}

\subsubsection{Identification of nitroreductases active with nil bystander antibiotics}

The 58 nitroreductase core library in the Ackerley lab (table 1.1) is the default library used to screen for nitroreductases that are active with nil bystander antibiotics. The core library contains 13 different families of enzymes, with the majority being from either the NfsA and NfsB families. The screening of this library with the 16 nil bystander antibiotic candidates (table 2.1 ) by growth inhibition assays (2.9.2) enabled the profiling of all nitroreductase/ nil bystander antibiotic combinations, and identification of combinations of nitroreductases and nil bystander antibiotics that might be useful for targeted cellular ablation.

\subsubsection{Identification of nitroreductases superior to NfsB_Ec for metronidazole activation}

The established applications of nitroreductases in developmental and regenerative biology led us to seek a better nitroreductase/ nil bystander antibiotic combination for targeted cell ablation.

Currently these studies employ the nitroreductase NfsB_Ec, and the nil bystander antibiotic metronidazole. The concentrations of metronidazole being used in these systems however, are extremely high and often toxic to the system, therefore finding a nitroreductase that has greater activity with metronidazole would be beneficial. Initial growth inhibition screens indicated that many nitroreductases in the 58 nitroreductase library had a much higher efficacy with metronidazole than NfsB_Ec which, at the lowest concentrations used, had essentially no effect. 
More detailed $\mathrm{IC}_{50}$ analyses indicated that $\mathrm{NfsB}_{-}$Vh had the best efficacy with metronidazole of all the nitroreductase candidates in our 58 nitroreductase library. Expression of NfsB_Vh in HCT-116 cells showed that this activated cell ablation capability was transferrable to mammalian cells.

The addition of FSL41, one of the masked fluorophores available in the lab, allowed fluorescence imaging of the cells containing NfsB_Vh. In both bacterial and mammalian cells, FSL41 was shown to be present in cell populations unchallenged with metronidazole, but upon the addition of metronidazole, and the subsequent ablation of the cell population, the fluorescence disappeared. This confirmed that we are able to use masked fluorophores to report on a cell population before and after the addition of a nil bystander antibiotic.

\subsubsection{Combined dual reporter/ ablation system}

The success of using a solely nitroreductase based system for the imaging/ ablation of a single cell population lead to the idea of a dual imaging/ ablation system - targeting two distinct populations of cells via two distinct nitroreductases, each able to activate one of two nil bystander antibiotics, and one of two masked fluorophores, with opposing activation profiles.

The complete screening of the 58 nitroreductase library against 16 nil bystander antibiotic candidates of interest enabled the generation of a colour coded heat-map to identify potential combinations for a more detailed evaluation by $\mathrm{IC}_{50}$ assays. Promising nitroreductases identified via this screening method were then able to be screened with masked fluorophores to identify any that could be used in a multiplex reporter/ cell ablation system.

Growth inhibition assays undertaken in bacterial cells confirmed the identification of nitroreductases with opposing activation profiles. For example, the nitroreductases NfsA_Li, NfsA_Pp, and NfsA_Ecaro are capable of activating tinidazole but not metronidazole, while $\mathrm{NfsB}$ _Vv is capable of activating metronidazole, but not tinidazole. The $\mathrm{IC}_{50}$ analysis of these nitroreductases with either tinidazole or metronidazole, indicated that NfsA_Li was the most effective activator of tinidazole, 
while at the same time having little to no ability to activate metronidazole. These results were the basis for selecting NfsA_Li and tinidazole in combination with NfsB_Vv and metronidazole as a promising dual nitroreductase ablation system. Unfortunately due to technical difficulties and time constraints, NfsA_Li was unable to be transfected into HEK293 cells. The $\mathrm{IC}_{50}$ analyses indicated that the next most effective activator of tinidazole that also exhibited minimal activation of metronidazole was NfsA_Pp.

The addition of metronidazole and tinidazole to HEK293 cells expressing either NfsB_Vv or NfsA_Pp mirrored the results that were observed in bacterial cells; this was a pleasing outcome, as not all nitroreductases are capable of being stably transfected and expressed in mammalian cells (Prosser et al., 2010; Prosser et al., 2013). The success of these two nitroreductases in activating distinct nil bystander antibiotics without substantial overlap, show a promising proof of principle that they will be able to be utilised for experimental work in mammalian based research although this has only been tested at a single cellular level it is a positive outcome likely to have practical applications in eukaryotic systems, especially zebrafish or Xenopus models.

The initial validation of a dual nitroreductase ablation system in both bacterial and mammalian cells led to the next step; adding a second factor to the system, a masked fluorophore, for imaging and reporting on ablation. Fluorophore screening was undertaken with a selection of masked fluorophores identified on the basis of previous work undertaken by Dr Horvat.

Again the initial screening was undertaken in bacterial cells, and promising combinations carried over into human cells. Results were consistent over both cell types. The nitroreductases that had initially indicated promise in terms of presenting opposing activation profiles with the nil bystander antibiotics, again showed opposing activation profiles to each other with masked fluorophores of different colours. The nitroreductases NfsA_Li and NfsA_Pp were identified as being capable of activating a green probe, FSL76 but not a red probe, FSL178; whereas NfsB_Vv was capable of activating FSL178, but not FSL76. 
Using a mixed population, mixed fluorophore model, and adding just one of the two possible nil bystander antibiotics, we observed that the masked fluorophores could report on the presence or absence (i.e. ablation) of cell populations expressing each nitroreductase. Thus, it appears that selective imaging and/or ablation of specific cells in a 2-way multiplexed nitroreductase expression system should be achievable in animal models.

\subsubsection{Niclosamide}

An interesting side story developed while screening the 58 nitroreductase library with the 16 nil bystander antibiotic candidates, when an anomaly in the activation profile of niclosamide was observed. In cell populations containing nitroreductases which are highly active with a particular prodrug, the population should be ablated upon the addition of that prodrug. Niclosamide however showed minimal growth inhibition with the most active nitroreductases in the library, and most tellingly, complete cell death with the empty pUCX plasmid. It appeared that the nitroreductases were conferring a protective effect on the cells.

Further investigation established that this protective effect was contingent on the $E$. coli host strain having its TolC exporter pump knocked out. Based on this observation, proof of principle experiments were conducted using solid media to show that niclosamide could be used to specifically select colonies over-expressing a functional nitroreductase enzyme. This interesting observation suggests that a single nitroreductase gene can act not only as a negative selection agent, but also a positive one, capable of defending the host cell against an otherwise toxic nitroaromatic insult. Possible applications of this technology are discussed in the following section.

\subsection{Future Directions}

\subsubsection{GDEPT}

The ablation of a tumour-targeting vector used in the proposed three-step approach to GDEPT is the final step in what could potentially be a ground-breaking new therapy for the treatment of cancer. It is an essential step as many of the proposed vectors for this technology are of pathogenic origin, making their ablation post treatment an essential step for FDA approval. 
This research has identified a number of lead nitroreductase/ nil bystander antibiotic combinations that could be tested for superior activity to $\mathrm{NfsB}$ _Ec/ metronidazole. For example, a lot of NfsA family members showed very high levels of activity with $\mathrm{N}$-benznidazole. Future experiments that could test the utility of these nitroreductase/ nil bystander antibiotic combinations for elimination of GDEPT vectors could be carried out in tumour xenograft models, using nitroreductase labelled bacterial or viral vectors, to confirm whether or not effective ablation of the vector can be achieved. If successful, this technology might have applications in other scenarios where an effective suicide gene is desirable as a safety control, for example in stem cell transplants, where the transplanted cell population could be rapidly ablated upon observing an adverse patient response.

\subsubsection{Developmental and regenerative biological studies}

The nitroreductase based reporter/ ablation systems tested in this thesis could also have numerous applications in developmental and regenerative biology. The ability to use these in a multiplex fashion potentially adds another layer of complexity, providing the ability to selectively target multiple cell types at any chosen time during development, either simultaneously or successively, and with the added advantage that is not present with gene knock out technology; the potential for reversal of the ablation after the removal of the nil bystander antibiotic in regenerative models.

The results obtained in this study are still preliminary. The system has shown very promising results in both bacterial and human cell populations, with initial proof of principle experiments carried out in bacterial cells and the results observed to generally be translatable into human cells. The next step for this work is to apply the same parameters in whole model organisms. The most obvious choices for this would either be in Xenopus or zebrafish, as these have already been explored for single cell population ablation in promoter driven nitroreductase / fluorescent protein systems. 


\subsubsection{Niclosamide as a tool for directed evolution}

The identification of a nil bystander antibiotic that had the opposite activation profile to that normally seen with nitroaromatic molecules - certainly to that observed with all other nil bystander antibiotics - led to the proposal of its use in the lab as a novel identifier of active nitroreductases, or its possible use as a tool for directed evolution. One application that is currently being investigated in the Ackerley lab is as a high throughput positive selection for identification of nitroreductases in metagenomic libraries (i.e. libraries of randomly purified and cloned DNA fragments sourced from a particular environment, e.g. soil). These libraries provide access to enzymes from the estimated $99 \%$ of microbes that cannot currently be cultured in the lab environment (Vartoukian et al., 2010).

A dual niclosamide and antibiotic prodrug selection could also allow the directed evolution of nitroreductases for loss of activity with that prodrug, while still retaining generic nitroreductase activity (i.e. the ability to protect the host cell against niclosamide). This could prove especially useful for tailoring the activity of nitroreductases to be used in the dual reporter/ ablation system. For example, whereas NfsB_Vv was strongly selective for metronidazole over tinidazole, NfsA_Pp was less selective for tinidazole over metronidazole (Figure 3.7), and thus was an imperfect partner for the dual ablation scenario. To eliminate this residual activity with metronidazole, one approach could be to use error-prone PCR to generate a large library of $n f s A \_P p$ gene variants, to clone this library into a tolC deleted strain of $E$. coli, to select for clones that can grow in the presence of both metronidazole and niclosamide, and finally to then screen these clones to identify individual mutants that have lost activity with metronidazole but can still activate tinidazole effectively.

\subsection{Concluding remarks}

Nitroreductases have proven to be versatile enzymes, capable of being used for a range of different activities, and in various fields through their unique ability to metabolise a vast range of nitro-quenched compounds. 
This study has built upon previous work in the Ackerley lab undertaken to exploit nitroreductases for their ability to activate nitro-quenched compounds, and has developed three novel ideas; a dual ablation/ reporter system for use in developmental and regenerative biological studies based solely around the activation properties of nitroreductases; a possible 2-way multiplex scenario, utilising two nitroreductases with opposing nil-bystander antibiotic/ masked fluorophore activation profiles; and a high throughput screening method utilising the anomaly observed in the activation profile of niclosamide.

Nitroreductases capable of superior activation of metronidazole to NfsB Ec, which is currently set as the 'gold-standard' of nitroreductase induced cell ablation in developmental biology studies, were identified. Likewise, promising candidates for activation of other nitro-quenched nil bystander antibiotics of interest, were uncovered. These results will help the expansion of nitroreductases for use in targeted cell ablation in both developmental biology, and a possible vector ablation system in GDEPT.

While these are the specific fields that this thesis has focused on, these results could have much further reaching impacts on various areas of research that utilise efficient nitroreductase catalysis.

The ability to partner masked fluorophores and nil bystander antibiotics both metabolised by the same nitroreductase will expand the utility of nitroreductases for targeted cell ablation, adding a built in reporter capability.

Niclosamide's positive selection for active nitroreductases has led to the possibility of developing a high throughput screening method to identify nitroreductases that are currently not accessible, due to the inability of their host organisms to be cultured in the lab. This could further expand the repertoire of nitroreductases available to different fields of science.

In summary, this thesis has demonstrated the catalytic flexibility of nitroreductase enzymes, as well as their potential to have a beneficial impact on numerous fields of science and medicine, most prominently GDEPT and developmental biology. 


\section{References}

Websites

http://www.drugbank.ca/drugs/DB00916, accessed 20.08.2013

http://www.drugbank.ca/drugs/DB00911 accessed 20.08.2013)

http://www.cancer.gov/drugdictionary?cdrid=39504 accessed 20.08.2013

http://www.cancer.gov/drugdictionary?cdrid=458064 accessed 20.08.2013

http://imaging.cancer.gov/programsandresources/fmiso-documentation accessed 02.09.2013

http://www.drugbank.ca/drugs/DB00614 accessed 20.08.2013

http://www.drugbank.ca/drugs/DB06803 accessed 20.08.2013

http://www.cancer.gov/clinicaltrials/search/view?cdrid=586650\&version=HealthProf essional accessed 20.08.2013

http://www.drugbank.ca/drugs/DB00698 accessed 20.08.2013

http://www.drugbank.ca/drugs/DB00336 accessed 20.08.2013

www.invitrogen.com/content/sfs/manuals/gatewayman.pdf accessed 29.09.2013

Articles

Barak, Y., Thorne, S.H., Ackerley, D.F., Lynch, S.V., Contag, C.H., and Matin, A. New enzyme for reductive cancer chemotherapy, YieF, and its improvement by directed evolution. Molecular Cancer Therapapeutics (2006). 5: 97-103 
Basu, P.P. Rifaximin therapy for metronidazole-unresponsive Clostridium difficile infection: a prospective pilot trial. Therapeutic advances in Gastroenterology (2010) 4: $221-225$

Benkli, K., Karaburun, A.C., Gundogdu-Karaburun, N., Demirayak, S., Guven, K., et al Synthesis and antimicrobial activities of some new nitroimidazole derivatives. Archives of Pharamacal Research (2003) 10: 773-777

Bhaumik, S., and Gambhir, S.S. Optical imaging of Renilla luciferase reporter gene expression in living mice (2002). PNAS 99: 377-382.

Bhaumik, S., Sekar, T.V., Depuy, J., Klimash, J., and Paulmurugan, R. Noninvasive optical imaging of nitroreductase gene-directed enzyme prodrug therapy system in living animals. (2011). Gene Therapy

Bremner, J.CAssessing the bioreductive effectiveness of the nitroimidazole RSU1069 and its prodrug RB6145: with particular reference to in vivo methods of evaluation. Cancer Metastasis Reviews (1993) 12: 177-193.

Brindle, K. New Approaches for Imaging Tumour Responses to Treatment. Nature Reviews (2008) 8: 94-107

Brown, J.M.Tumor hypoxia in cancer therapy. Methods in Enzymology (2007) 435:297-321

Bryant, D.W., McCalla, D.R., Leeksma, M., and Laneuville, P. Type I nitroreductases of Escherichia coli. Canadian Journal of Microbiology (1981) 27, 8186.

Brown, J.M., Giaccia, A.J. The Unique Physiology of Solid Tumors: Opportunities (and Problems) for Cancer Therapy. Cancer Research (1998) 58: 1408

Brown, J. M., Wilson, W. R. Exploiting Tumour Hypoxia in Cancer Treatment. Nature Reviews (2004) 4: 437-447 
Castro, J.A., Diaz de Toranzo, E.G. Toxic effects of nifurtimox and benznidazole, two drugs used against American trypanosomiasis (Chagas' disease). Biomedical and Environmental Sciences (1988) 1: 19-33

Chen, Y., Hu, L. Design of anticancer prodrugs for reductive activation. Medicinal Research Reviews (2009) 29: 29-64

Chen, S., Knox, R., Wu, K., Deng, P., S., Zhou, D., Bianchet, M. A., Amzel, L. M. Molecular Basis of the Catalytic Differences among DT-diaphorase of Human, Rat, and Mouse. The Journal of Biological Chemistry (1997) 272: 1437-1439.

Christofferson, A, Wilkie. J Mechanism of CB1954 reduction by Escherichia coli nitroreductase. Biochemical Society Transactions (2009) 37: 413

Croce, C.M. Molecular Origins of Cancer Oncogenes and Cancer. The New England Journal of Medicine (2008) 358: 502-511

Curado S, Stainier DY, Anderson RM: Nitroreductase-mediated cell/tissue ablation in zebrafish: a spatially and temporally controlled ablation method with applications in developmental and regeneration studies. Nature protocols (2008) 3(6):948-954

Curado, S., Anderson, R.M., Jungblut, B., Mumm, J., Schroeter, E., Stainer, Y.R., Conditional Targeted Cell Ablation in Zebrafish: A New Tool for Regeneration Studies. Developmental Dynamics (2007) 236: 1025-1035

Dachs, G. U., Hunt, M. A., Syddall, S., Singleton, D. C., Patterson A. V. Bystander or No Bystander for Gene Directed Enzyme Prodrug Therapy. Molecules (2009) 14: $4517-4545$

Davison JM, Akitake CM, Goll MG, Rhee JM, Gosse N, Baier H, Halpern ME, Leach SD, Parsons MJ: Transactivation from Gal4-VP16 transgenic insertions for tissuespecific cell labeling and ablation in zebrafish. Developmental biology (2007) 304(2):811-824. 
Deacon, J.M., Holliday, S.B., Ahmed, I., Jenkins, T.C. Experimental

pharmacokinetics of RSU-1069 and its analogues: high tumor/plasma ratios (1986)

International journal of radiation oncology, biology, physics 12: 1087-1090

Denny, W.A. The role of hypoxia-activated prodrugs in cancer therapy. The Lancet Oncology (2000). 1: 25-29.

Denny, W.A. Prodrugs for Gene-Directed Enzyme-Prodrug Therapy (Suicide Gene Therapy). Journal of Biomedical Biotechnology (2003) 1: 48-70

Denny WA, Wilson WR. Considerations for the Design of Nitrophenyl Mustards as Agents with Selective Toxicity for Hypoxic Tumor Cells. Journal of Medicinal Chemistry (1986) 29: 879-887

DeVita, V. T., Chu, E. A History of Cancer Chemotherapy. Cancer Research (2008) 68: $8643-8653$

Greco, O., Dachs, G. U., Gene Directed Enzyme/Prodrug Therapy of Cancer:

Historical Appraisal and Future Prospectives. Journal of Cell Physiology (2001) 187: $22-36$

Gunay, N.S., Capan, G., Ulusoy, N., Ergenc, N., Otuk, G., Kaya, D. 5-Nitroimidazole derivatives as possible antibacterial and antifungal agents. Farmaco (1999) 54: 826831

Hartley, J.L., Temple, G.F., Brasch, M.A. DNA cloning using in vitro site-specific recombination. Genome Research (2000) 10: 1788-1795 
Kaya, F., Mannioui, A., Chesneau, A., Sekizar, S., Maillard, E., Ballagny, C., HouelRenault, L., Dupasquier, D., Bronchain, O., Holtzmann, I., Desmazieres, A., Thomas, J.L., Demeneix, B.A., Brophy, P.J., Zalc, B., Mazabraud, A.. Live imaging of targeted cell ablation in Xenopus: a new model to study demyelination and repair. The Journal of Neuoroscience (2012) 37: 12885-12895

Kennedy AS, Raleigh JA, Perez GM, Calkins DP, Thrall DE, Novotny DB, Varia MA. Proliferation and Hypoxia in Human Squamous Cell Carcinoma of the Cervix: First Report of Combined Immunohistochemical Assays. International Journal of Radiation Oncology, Biology, Physics (1997) 37: 897-905

Komar, G., Seppanen, M., Eskola, O., Lindholm, P., Gronroos, T. J., Forsback, S., Sipila, H., Evans, S. M., Solin, O., Minn, H. ${ }_{-}{ }^{18}$ F-EF5: a new PET tracer for imaging hypoxia in head and neck cancer. Journal of Nuclear Medecine (2008) 49: 1944-1951

Koronakis, V., Eswaran, J., Hughes, C. Structure and function of TolC: the bacterial exit duct for proteins and drugs. Annual Review of Biochemistry (2004) 73: 467-489

Kremers, G.J., Gilbert, S.G., Cranfill, P.J., Davidson, M.W., Piston, D.W.

Fluorescent proteins at a glance. Journal of Cell Science (2011) 124: 157-160

Löfmark, S., Edlund, C., Nord, C.E. Metronidazole Is Still the Drug of Choice for Treatment of Anaerobic Infections. Clinical Infectious Diseases (2010) 50: S16-S23

McCormick, F. Cancer Gene Therapy: Fringe or Cutting Edge? Nature Reviews (2001) 1: 130-141

Moreno, S.N., Docampo, R. Mechanism of toxicity of nitro compounds used in the chemotherapy of trichomoniasis. Environmental Health Perspectives (1985) 64: 199208

Muller, M. Mode of action of metronidazole on anaerobic bacteria and protozoa. Surgery (1983) 93: 165-171 
Osada, T., Chen, M., Yang, X.Y., Vandeusen, J.B., Hsu, D., Clary, B.M., Clary, T.M., Chen, W., Morse, M.A., Lyerly, H.K., Antihelminth Compound Niclosamide Downregulates Wnt Signaling and Elicits Antitumor Responses in Tumors with Activating APC Mutations (2011) Cancer Research 12: 4172-4182

Padera, T., Stoll, B., Tooredman, J., Capen, D., di Tomaso, E., Jain, R. Pathology: Cancer Cells Compress Intratumour Vessels. Nature (2004) 427: 695

Parkinson, G.N., Skelly, J.V., Neidle, S. Crystal structure of FMN-dependent nitroreductase from Escherichia coli B: a prodrug-activating enzyme. Journal of Medicinal Chemistry (2000) 43: 3624-3631

Paterson, E.S., Boucher, S.E., Lambert, I.B. Regulation of the $n f s A$ Gene in Escherichia coli by SoxS. Journal of Bacteriology (2002) 1: 51-8

Patridge, E.V., Ferry, J.G. WrbA from Escherichia coli and Archaeoglobus fulgidus is an NAD(P)H:quinone oxidoreductase. Journal of bacteriology (2006) 188: 34983506

Patterson, A.V., Ferry, D.M., Edmunds, S.J., Gu, Y., Singleton, R. S., Patel, K., Pullen, S. M., Hicks, K. O., Syddall, S. P., Atwell, G. J., Yang, S., Denny, W. A., Wilson, W. R. Cancer Therapy: Preclinical Mechanism of Action and Preclinical Antitumor Activity of the Novel Hypoxia-Activated DNA Cross-Linking Agent PR104. Clinical Cancer Research (2007) 13: 3922-3932

Thanabalu T., Koronakis E., Hughes C., Koronakis V. Substrate-induced assembly of a contiguous channel for protein export from E.coli: Reversible bridging of an innermembrane translocase to an outer membrane exit pore. EMBO Journal (1998) 17: $6487-6496$

Theys, O Pennington' L Dubois' G Anlezark, T Vaughan' A Mengesha' W Landuyt, J Anne, P J Burke, P Dûrre, B G Wouters, N P Minton and P Lambin Repeated cycles of Clostridium-directed enzyme prodrug therapy result in sustained antitumour effects in vivo British Journal of Cancer (2006) 95: 1212-1219. 
Pisharath, P., Parsons, M.J., Lieschke, G.J., Nitroreductase-Mediated Cell Ablation in Transgenic Zebrafish Embryos (2009) Zebrafish, Methods in Molecular Biology, vol. 546

Prasher, D.C., Eckenrode, V.K., Ward, W.W., Prendergast, F.G., Cormier, M.J.

Primary structure of the Aequorea victoria green-fluorescent protein. Gene (1992)

111: $229-233$

Prosser, G.A., Copp, J. N., Mowday, A.M., Guise, C.P., Syddall, S.P., Williams, E.M., Horvat, C. N., Swe, P.M., Ashoorzadeh, A., Denny, W. A., Smaill, J. B., Patterson, A. V., Ackerley, D.F. Creation and screening of a multi-family bacterial oxidoreductase library to discover novel nitroreductases that efficiently activate the bioreductive prodrugs CB1954 and PR-104A. Biochemical Pharmacology (2013) 85: $1091-1103$

Prosser, G. A., Copp, J. N., Syddall, S. P., Williams, E.M., Smaill, J. B., Wilson, W.R., Patterson, A.V., Ackerley, D. F. Discovery and Evaluation of Escherichia coli Nitroreductases that Activate the Anti-cancer Prodrug CB1954. Biochemical Pharmacology (2010b) 79: 678-687

Prosser, G. A., Patterson, A. V., Ackerley, D. F. UvrB Gene Deletion Enhances SOS Chromotest Sensitivity for Nitroreductases that Preferentially Generate the 4hydroxylamine Metabolite of the Anti-cancer Prodrug CB1954. Journal of Biotechnology (2010a) 150: 190-194

Rainov, N. G. A Phase III Clinical Evaluation of Herpes Simplex Virus Type 1 Thymidine Kinase and Ganciclovir Gene Therapy as an Adjuvant to Surgical Resection and Radiation in Adults with Previously Untreated Glioblastoma Multiforme. Human Gene Therapy (2000) 11: 2389-2401

Sack, U., Walther, W., Scudiero, D., Selby, M., Kobelt, D., Lemm, M., Fichtner, I., Schlag, P.M., Shoemaker, R. H., Stein, U. Novel Effect of Antihelminthic Niclosamide on S100A4-Mediated Metastatic Progression in Colon Cancer. Journal of the National Cancer Institute (2011) 13: 1018-36 
Sasaki, Y., Sone, T., Yoshida, S., Yahata, K., Hotta, J., Chesnut, J.D., Honda, T., Imamoto, F. Evidence for high specificity and efficiency of multiple recombination signals in mixed DNA cloning by the Multisite Gateway system. Journal of Biotechnology (2004) 107: 233-243

Sawyer, P.R., Brogden, R.N., Pinder, R.M., Speight, T.M., Avery, G.S., Tinidazole: A Review of its Antiprotozoal Activity and Therapeutic Efficacy. Drugs (1976) 6: $423-40$

Schaller, T., Appel, N., Koutsoudakis, G., Kallis, S., Lohmann, V., Pietschmann, T., Bartenschlager, R. Analysis of Hepatitis C Virus Superinfection Exclusion by Using Novel Fluorochrome Gene-Tagged Viral Genomes Journal of Virology (2007) 9: $4591-4603$

Schmidt, C. Amgen spikes interest in live virus vaccines for hard-to-treat cancers. Nature Biotechnology (2011) 29(4):295-296

Siim BG, Denny WA, Wilson WR. Nitro reduction as an electronic switch for bioreductive drug activation. Oncology Research (1997) 9(6-7):357-369

Singleton, D.C., Li, D., Bai, S.Y., Syddall, S.P., Smaill, J.B., Shen, Y., Denny, W.A., Wilson, W.R., Patterson, A.V. The Nitroreductase Prodrug SN 28343 Enhances the Potency of Systemically Administered Armed Oncolytic Adenovirus ONYX411NTR. Cancer Gene Therapy (2007) 14: 953-967

Steib, A., Jacoberger, B., Von Bandel, M., Beck, F., Beller, J.P., Boudjema, K., Koffel, J.C., Otteni, J.C. Concentrations in plasma and tissue penetration of ceftriaxone and ornidazole during liver transplantation. Antimicrobial Agents and Chemotherapy (1993) 37: 1873-1876

Streker, K., Freiberg, C., Labischinski, H., Hacker, J., Ohlsen, K.. Staphylococcus aureus NfrA (SA0367) is a flavin mononucleotide-dependent NADPH oxidase involved in oxidative stress response (2005) 187: 2249-2256 
van Rossum, T., Kengen, S.W., van der Oost, J. Reporter-based screening and selection of enzymes FEBS Journal (2013) 13: 2979-2996

Vartoukian SR, Palmer RM, Wade WG. Strategies for culture of 'unculturable' bacteria Federation of European Microbiological Societies Microbiology Letters (2010) 309:1-7.

Vass, S.O., Jarrom, D., Wilson, W.R., Hyde, E.I., and Searle, P.F. E. coli NfsA: an alternative nitroreductase for prodrug activation gene therapy in combination with CB1954 (2009) British Journal of Cancer 100, 1903-1911.

White, D.T. Mumm, J, S. The nitroreductase system of inducible targeted ablation facilitates cell-specific regenerative studies in zebrafish. Methods (2013) pub. Online March 2013. In Press.

White, Y.A.R., Woods, D.C., Wood, A.W., A transgenic zebrafish model of targeted oocyte ablation and de novo oogenesis. Developmental Dynamics (2011) 240: 1929 1937

Whiteway, J., Koziarz, P., Veall, J., Sandhu, N., Kumar, P., Hoecher, B., Lambert, I.B. Oxygen-Insensitive Nitroreductases: Analysis of the Roles of nfs A and nfsB in Development of Resistance to 5-Nitrofuran Derivatives in Escherichia coli. Journal of Bacteriology (1998) 21: 5529-5539

Wilson, W.R., Pullen, S.M., Hogg, A., Helsby, N.A., Hicks, K.O., Denny, W.A. Quantitation of bystander effects in nitroreductase suicide gene therapy using threedimensional cell cultures Cancer Research (2002) 5: 1425-32

Wu, B., Piatkevich, K.D., Lionnet, T., Singer, R.H., Verkhusha, V.V. Modern Fluorescent Proteins and Imaging Technologies to Study Gene Expression, Nuclear Localization, and Dynamics. Current Opinion in Cell Biology (2011) 23: 310-317

Zenno, S., Koike, H., Tanokura, M., Saigo, K. Gene cloning, purification, and 
characterisation of $\mathrm{NfsB}$, a major oxygen-insensitive nitroreductase from Escherichia coli, similar in biochemical properties to FRaseI, the major flavin reductase in Vibrio fischeri. Journal of Biochemistry (1996) 120: 736-744

Zhao XF, Ellingsen S, Fjose A. Labelling and targeted ablation of specific bipolar cell types in the zebrafish retina. (2009) BMC Neuroscience 10: 107 